


\title{
Der Einfluss der Glutamatdehydrogenasen auf die Verknüpfung des Kohlenstoff- und Stickstoffstoffwechsels in Bacillus subtilis
}

\author{
Dissertation zur Erlangung des Doktorgrades \\ der Mathematisch-Naturwissenschaftlichen Fakultäten \\ der Georg-August-Universität zu Göttingen
}

vorgelegt von

Katrin Gunka

aus Cuxhaven

Göttingen 2010 
D7

Referent:

Prof. Dr. Jörg Stülke

Korreferent:

PD Dr. Michael Hoppert

Tag der mündlichen Prüfung: $\quad$ 26.01.2011 
"Ohne Spekulation gibt es keine neue Beobachtung."

(Charles Darwin) 


\section{Danksagung}

Als Erstes möchte ich mich bei Prof. Dr. Jörg Stülke für die Ermöglichung meiner Doktorarbeit bedanken. Vielen Dank für die hervorragende Unterstützung, das stetige Interesse und dafür, dass ich mich immer gut aufgehoben fühlte!

Nicht nur für die freundliche Übernahme des Korreferates, sondern auch für sein Interesse und seine Hilfsbereitschaft möchte ich mich bei PD Dr. Michael Hoppert sehr bedanken.

Prof. Dr. Heinz Neumann, PD Dr. Boris Görke, PD Dr. Rolf Daniel und Prof. Dr. Botho Bowien gilt mein Dank für Ihre Teilnahme an meiner Prüfungskommission.

Unseren Partnern aus Newcastle Prof. Dr. Rick J. Lewis, Dr. Joseph A. Newman, Dr. Lorraine Hewitt und Dr. Cecilia Rodrigues danke ich für die Kristallisation der Glutamatdehydrogenasen.

Außerdem möchte ich mich besonders bei Fabian M. Commichau bedanken. Er hat mir sein Wissen und seine Leidenschaft für das Suchen von Papillen vererbt!

Meinen ehemaligen Kollegen, Claudine Hames und Sebastian Hübner, danke ich dafür, dass Sie mir vieles beigebracht haben und wir immer noch so viel Spaß zusammen haben können.

Bei all meinen lieben (ehemaligen) Kollegen, Nico Pietack, Arne Schmeisky, Lope Flórez, Fabian Rothe, Frederik Meyer, Martin Lehnik, Hinnerk Eilers und Sebastian Schmidl, möchte ich mich dafür bedanken, dass ich immer gerne zur Arbeit ging. Egal ob gute oder weniger gute Ergebnisse auf mich gewartet haben, ich wusste, dass auf Euch Verlass ist, auch außerhalb der Arbeit!

Mein besonderer Dank gilt Tini Diethmaier, nicht nur für die weibliche Unterstützung ;-), sondern vor allem für eine wirklich schöne Zeit!

Christina Herzberg möchte ich dafür danken, dass Sie so unglaublich engagiert Ihr Bestes gibt und das Bacillus Labor (nicht nur in wissenschaftlichen Belangen) am Laufen hält.

Dr. Petra Neumann-Staubitz, Julia Busse und Sabine Lentes danke ich für die gute Zusammenarbeit und die netten Gespräche während meiner Doktorarbeit. Bärbel Herbst danke ich dafür, dass Sie alles möglich macht, um uns die Arbeit zu erleichtern.

Meinem ehemaligen Diplomanden Stefan Tholen danke ich für die schöne gemeinsame Zeit im Labor und dafür, dass er vieles zum GudB-Projekt beigetragen hat. 
Bei meinen Praktikanten Jens Baumbach, Rafik Neme, Bernard Freytag und meinen Bachelorstudentinnen Kristin Kaiser und Victoria Hentschke möchte ich mich für den Beitrag an dieser Arbeit und meiner persönlichen Entwicklung im „BetreuungsBusiness" bedanken.

Bei allen ehemaligen Diplom- und Bachelorstudent(inn)en, insbesondere Henrike Pförtner und Chris Zschiedrich bedanke ich mich für viel Spaß im Labor.

AG Görke und AG Hoppert, besonders Denise Lüttmann und Jens Landmann, möchte ich für die gute Zusammenarbeit und hilfreichen Gespräche danken.

Meinen Freunden, die ich hier in Göttingen kennengelernt habe, gilt ein großes Dankeschön, da Sie maßgeblich dazu beigetragen haben, dass ich mich hier wohlfühlte und auch mal die Arbeit vergessen konnte ;-).

Meiner ganzen Familie, insbesondere meinen lieben Eltern und meiner Tante Eleonore, möchte ich herzlich danken, dass Sie mich immer unterstützt, mit mir gelitten und sich vor allem mit mir gefreut haben.

Christoph möchte ich so sehr danken, dass wir uns unseren Traum zusammen erfüllt haben. Ich freue mich auf viele weitere Träume in unserer Zukunft! 


\section{Table of Contents}

List of Abbreviations............................................................................................. III

List of Publications ................................................................................VI

Summary .................................................................................................................... 1

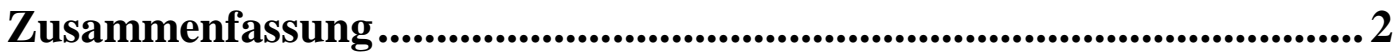

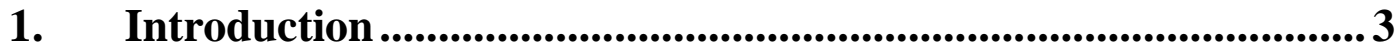

1.1. The general role of glutamate in life................................................... 4

1.1.1. Glutamate metabolism in Bacillus subtilis ............................................. 5

1.1.2. The regulation of glutamate metabolism in B. subtilis ............................ 7

1.1.3. Impact of glutamate metabolism on phenotypical features of B. subtilis.... 12

1.1.4. The importance of glutamate homeostasis.............................................. 14

1.2. The controversy of adaptive mutations ....................................................... 16

1.2.1. The frequency of mutations .................................................................. 17

1.3. Transcription-associated DNA repair ................................................ 18

1.3.1. The role of Mfd in cellular physiology .................................................. 18

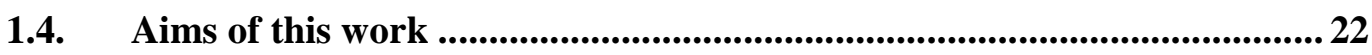

\section{Chapter 2}

Functional dissection of a trigger enzyme: Mutations of the Bacillus subtilis glutamate dehydrogenase RocG that affect differentially its catalytic activity and regulatory properties ................................................. 23

\section{Chapter 3}

Analysis of the RocG-GItC interaction................................................52

\section{Chapter 4}

A high-frequency mutation in Bacillus subtilis: Requirements for the decryptification of the gudB glutamate dehydrogenase gene .................. 66 


\section{Chapter 5}

Further factors involved in the Mfd-dependent decryptification of the gudB allele in Bacillus subtilis .......................................................88 8

\section{Chapter 6}

SPABBATS: A pathway-discovery method based on Boolean satisfiability that facilitates the characterization of suppressor mutants

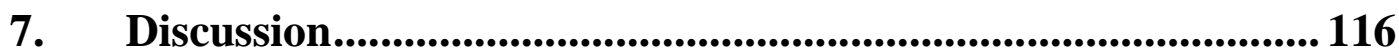

7.1. The emergence of suppressor mutations in response to glutamate

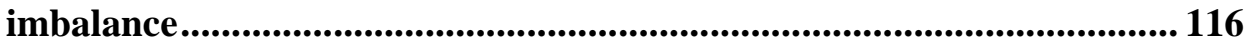

7.2. The cryptification of the gudB gene.......................................................... 117

7.3. The Mfd protein is required for the gudB decryptification.................... 120

7.4. A perfect direct repeat is required for the efficient $g u d B$ decryptification...................................................................................... 121

7.5. The separation of enzymatic and regulatory activities of RocG............. 125

7.6. A new model of RocG-GltC interaction .................................................. 126

7.7. Perspectives ........................................................................................... 130

8. References ............................................................................ 131

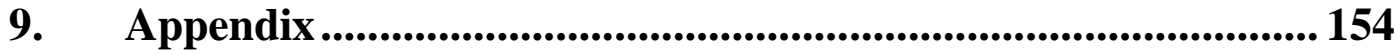

9.1. Oligonucleotides ........................................................................................... 154

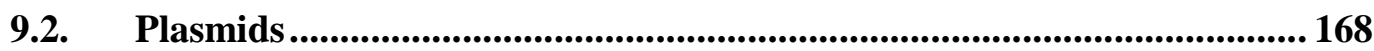

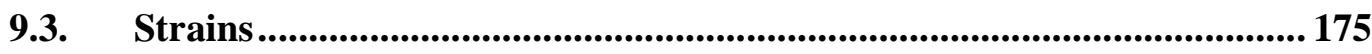




\section{List of Abbreviations}

\begin{tabular}{|c|c|}
\hline$\%(\mathrm{vol} / \mathrm{vol})$ & $\%$ (volume/volume) \\
\hline$\%(\mathrm{wt} / \mathrm{vol})$ & $\%$ (weight/volume) \\
\hline 3-m-2-o-b-ate & 3-methyl-2-oxo-butanoate \\
\hline 4-m-2-o-p-ate & 4-methyl-2-oxo-pentanoate \\
\hline ADP & adenosine diphosphate \\
\hline Amp & ampicillin \\
\hline AP & alkaline phospatase \\
\hline ATP & adenosine triphosphate \\
\hline$B$. & Bacillus/Bordetella \\
\hline $\mathrm{B} 2 \mathrm{H}$ & bacterial two-hybrid \\
\hline$C$ & Clostridium/Corynebacterium \\
\hline cAMP & cyclic AMP \\
\hline CcpA & catabolite control protein A \\
\hline \multirow[t]{2}{*}{$\mathrm{CDP}^{*}$} & disodium 2-chloro-5-(4-methoxyspiro \{1,2-dioxetane-3,2'-(5'-chloro) \\
\hline & tricyclo[3.3.1.13,7]decan $\}-4-y l)$ phenyl phosphate \\
\hline $\mathrm{Cm}$ & chloramphenicol \\
\hline cre & catabolite responsive element \\
\hline DNA & deoxyribonucleic acid \\
\hline E. & Escherichia \\
\hline EDTA & ethylenediaminetetraacetic acid \\
\hline Erm & erythromycin \\
\hline EMSA & electrophoretic mobility shift assay \\
\hline FA & formaldehyde \\
\hline FAD & flavin adenine dinucleotide (oxidized) \\
\hline FADH & flavin adenine dinucleotide (reduced) \\
\hline FBA & Flux Balance Analysis \\
\hline Fig. & figure \\
\hline fwd & forward \\
\hline GDH & glutamate dehydrogenase \\
\hline Glc & glucose \\
\hline
\end{tabular}




\begin{tabular}{|c|c|}
\hline GOGAT & glutamine-oxoglutarate-aminotransferase \\
\hline GS & glutamine synthetase \\
\hline $\operatorname{IgG}$ & Immunglobulin $\mathrm{G}$ \\
\hline IPTG & isopropyl- $\beta$-D-thiogalactopyranoside \\
\hline $\mathrm{k}_{\mathrm{m}}$ & Michaelis constant \\
\hline LB & Luria Bertani (medium) \\
\hline LFH-PCR & Long Flanking Homology PCR \\
\hline Lin & Lincomycin \\
\hline MILP & mixed-integer linear programming \\
\hline MOMA & minimization of metabolic adjustment \\
\hline mRNA & messenger RNA \\
\hline NAD & nicotinamide adenine dinucleotide (oxidized) \\
\hline $\mathrm{NADH}$ & nicotinamide adenine dinucleotide (reduced) \\
\hline $\mathrm{NADP}^{+}$ & nicotinamide adenine dinucleotide phosphate (oxidized) \\
\hline NADPH & nicotinamide adenine dinucleotide phosphate (reduced) \\
\hline NER & nucleotide excision repair \\
\hline $\mathrm{OD}_{\mathrm{x}}$ & optical density, measured at the wavelength $\lambda=\mathrm{x} n \mathrm{~m}$ \\
\hline ONPG & ortho-Nitrophenyl- $\beta$-galactoside \\
\hline ori & origin of replication \\
\hline$P$ & promoter \\
\hline PAGE & polyacrylamide gel electrophoresis \\
\hline PCR & polymerase chain reaction \\
\hline PEG & polyethylene glycol \\
\hline $\mathrm{pH}$ & power of hydrogen \\
\hline PVDF & polyvinylidene difluoride \\
\hline r.m.s.d. & root mean square deviation \\
\hline rev & reverse \\
\hline RNA & ribonucleic acid \\
\hline ROOM & regulatory on/off minimization \\
\hline S & Succinate \\
\hline$S$. & Saccharomyces \\
\hline SASP & small acid soluble proteins \\
\hline SAT & Boolean satisfiability \\
\hline
\end{tabular}


SD

SDS

SigL

SP

SPABBATS

Spec

SPINE

SPR

Tab.

TCA

TCR

TCRF

Tet

Tris

U

WT

X-Gal

YFP
Shine-Dalgarno

sodium dodecyl sulfate

sigma factor $\mathrm{L}$

sporulation medium

shortest pathway between the basal and target sets

spectinomycin

Strep-protein interaction experiment

surface plasmon resonance

table

tricarboxylic acid

transcription-coupled nucleotide excision repair

transcription repair coupling factors

tetracycline

tris(hydroxymethyl)aminomethane

units

wild type

5-bromo-4-chloro-3-indolyl- $\beta$-D-galactopyranoside

yellow fluorescent protein 


\section{List of Publications}

Gunka, K., Newman, J. A., Commichau, F. M., Herzberg, C., Rodrigues, C., Hewitt, L., Lewis, R. J. \& Stülke, J. (2010) Functional dissection of a trigger enzyme: Mutations of the Bacillus subtilis glutamate dehydrogenase RocG that affect differentially its catalytic activity and regulatory properties. J. Mol. Biol. 400: 815-827.

Flórez, L. A., Gunka, K., Polanía, R., Tholen, S. \& Stülke, J. SPABBATS: A pathway-discovery method based on Boolean satisfiability that facilitates the characterization of suppressor mutants. BMC Systems Biology submitted

Gunka, K., Tholen, S., Neme, R., Freytag, B., Commichau, F. M., Herzberg, C. \& Stülke, J. A high-frequency mutation in Bacillus subtilis: Requirements for the decryptification of the $\operatorname{gudB}$ glutamate dehydrogenase gene. Mol. Microbiol. submitted 


\section{Summary}

Glutamate is the central amino group donor for all nitrogen containing compounds in the cell. In the Gram-positive soil-dwelling bacterium Bacillus subtilis glutamate is exclusively synthesized by the combined reactions of the glutamine synthetase and the glutamate synthase. Since the synthesis of glutamate requires 2-oxoglutarate that is derived from the tricarboxylic acid cycle, glutamate metabolism is an important intersection between the carbon and the nitrogen metabolism. Thus, the expression of the biosynthetic enzymes of the glutamate metabolism is highly regulated. The opposite reaction, the degradation of glutamate to 2-oxoglutarate is catalyzed by the glutamate dehydrogenase RocG. This enzyme is not capable of glutamate synthesis probably due to its low affinity for ammonium. In addition to its enzymatic function, RocG triggers the activity of the transcriptional regulator GltC that is essential for the expression of the gltAB operon encoding the glutamate synthase.

In this work, mutant variants of the RocG protein were isolated and analyzed. Single amino acid exchanges uncoupled the two functions of the RocG protein. One class of mutants is severely impaired in its catalytic activity but strongly inhibits the GltC protein, thus preventing the expression of the gltAB operon. The second class completely lost the ability to inhibit GltC but retained full enzymatic activity. The data provide an insight into the regulatory mechanism of the RocG-GltC interaction.

B. subtilis encodes a second glutamate dehydrogenase, GudB. In the laboratory strain 168 the $\operatorname{gudB}$ gene is cryptic due to a direct repeat of nine base pairs leading to a duplication of three amino acids in the active center of the enzyme. In a roc $G$ mutant strain the $g u d B$ allele is readily decryptified upon growth on complex medium by the precise deletion of one half of the direct repeat.

This work shows that the $\operatorname{gudB}$ mutation occurs at an extremely high rate of $10^{-4}$. Evidence was provided that a perfect direct repeat is crucial for the rapid decryptification of $\operatorname{gudB}$. Moreover, by using an artificial mutagenesis system it turned out that transcription is involved in the high mutation rate of the $\operatorname{gudB}$ gene. Indeed, the transcription repair coupling factor Mfd is required for the decryptification of the $g u d B$ gene.

The results of this work emphasize the importance of glutamate homeostasis in B. subtilis. 


\section{Zusammenfassung}

Glutamat ist der zentrale Aminogruppendonor für alle stickstoffhaltigen Verbindungen in der Zelle. In dem Gram-positiven Bodenbakterium Bacillus subtilis wird Glutamat ausschließlich in den gekoppelten Reaktionen der Glutaminsynthetase und der Glutamatsynthase synthetisiert. Da für den Aufbau von Glutamat 2-Oxoglutarat aus dem Citrat Zyklus benötigt wird, stellt die Glutamatsynthese einen wichtigen Knotenpunkt zwischen dem Kohlenstoff- und Stickstoffstoffwechsel dar. Deshalb ist die Expression der beteiligten Enzyme streng reguliert. Der Abbau von Glutamat wird von der Glutamatdehydrogenase RocG katalysiert. Durch die geringe Affinität zu Ammonium kann dieses Enzym nicht die Synthese von Glutamat katalysieren. Neben der enzymatischen Funktion reguliert RocG die Aktivität des Transkriptionsregulators GltC, der bedeutend für die Expression der Glutamatsynthase ist.

In dieser Arbeit wurden mutierte RocG-Varianten isoliert und charakterisiert. Durch einen Aminosäureaustausch wurden die beiden Funktion von RocG voneinander getrennt. Eine Klasse von Varianten inaktiviert GltC sehr stark und ist in ihrer zeigt enzymatischen Aktivität schwer beeinträchtigt. Die zweite Klasse kann GltC nicht mehr inhibieren, zeigt aber enzymatische Aktivität. Diese Ergebnisse geben einen Einblick in den regulatorischen Mechanismus der RocG-GltC Interaktion.

B. subtilis 168 kodiert für eine zweite Glutamatdehydrogenase, GudB. Im Laborstamm 168 ist das $g u d B-G e n$ durch eine direkte Wiederholung von neun Basenpaaren kryptisch. Beim Wachstum einer rocG-Mutante auf Komplexmedium wird das gudB-Allel schnell dekryptifiziert, wobei immer eine Hälfte der Sequenzwiederholung deletiert wird.

In dieser Arbeit wurde gezeigt, dass die gudB-Mutation mit einer extrem hohen Rate von $10^{-4}$ auftritt. Außerdem wurde bewiesen, dass eine perfekte Basenwiederholung für die hohe Mutationsrate notwendig ist. Darüber hinaus zeigte sich in einem artifiziellen Mutagenese-System, dass die Transkription für die hohe Mutationsrate des gudB-Gens notwendig ist. Das Mfd Protein, welches die Transkription mit der DNA Reparatur koppelt, spielt eine entscheidende Rolle bei der Dekryptifizierung des $g u d B$ Gens.

Die Ergebnisse dieser Arbeit unterstreichen die herausragende Bedeutung der Glutamathomöostase in B. subtilis. 


\section{Introduction}

\section{Prologue}

Bacillus subtilis is a rod-shaped bacterium that was first described as Vibrio subtilis by C. G. Ehrenberg in 1835 but renamed in Bacillus subtilis by J. F. Cohen in 1872 (Gordon et al., 1981). B. subtilis belongs to the phylum of the Firmicutes with low GC content and has become famous as a model organism for Gram-positive sporeforming bacteria. Naturally, the bacterium is common in the soil. In this environment the bacterium has to cope with fluctuating conditions which probably have led to its ability to adapt rapidly to stress conditions (Boylan et al., 1993; Helmann et al., 2001; Hecker et al., 2007). These survival strategies as well as the close relation to pathogens such as $B$. anthracis directed the interest of research on B. subtilis. The secretion of proteases, attractive for the industry, the production of antibiotics and the development of competence are only a few aspects worth mentioning of the complex reactions to unfavorable growth conditions of B. subtilis (Hamoen et al., 2003). The mechanism of DNA uptake and integration of this DNA in its genome, referred to as competence, allows genetic manipulation of $B$. subtilis (Harwood, 1990). The whole genome (4.2 Mbp) of B. subtilis was sequenced in 1997 (Kunst et al., 1997) and a resequencing approach in 2009 provides even more reliable sequence information now (Barbe et al., 2009). As the knowledge on B. subtilis has rapidly grown, new platforms were developed to manage all present and upcoming information. Recently, two complementary online databases namely SubtiWiki and SubtiPathways were published (Flórez et al., 2009; Lammers et al., 2010). These tools represent a compact accessibility of data which will benefit to the further research on B. subtilis. 


\subsection{The general role of glutamate in life}

The amino acid glutamate plays an important role in all domains of life. Glutamate fulfills a great variety of functions in all organisms. It serves as the major amino group donor for all nitrogen containing compounds of the cell. Glutamate is the most important amino group shuttle for the synthesis of amino acids, amino sugars and nucleotides (Ikeda et al, 1996; Reitzer, 2003). In Escherichia coli, 88\% of the nitrogen in the biomass is derived from glutamate mainly via transamination reactions and only $12 \%$ is supplied by glutamine (Reitzer, 1996; Goss et al., 2001). Also in other microorganisms e.g. the yeast Saccharomyces cerevisiae, glutamate is the source of about $80 \%$ of the cellular nitrogen (Magasanik, 2003). The importance of glutamate in physiological processes is reflected by its high abundance. In E. coli, glutamate is the most abundant metabolite with over $40 \%$ of the total detectable intracellular metabolome (Bennett et al., 2009). Furthermore, glutamate is also present at the highest concentration of all metabolites in the opportunistic human pathogen Pseudomonas aeruginosa (Frimmersdorf et al., 2010). Although glutamate seems to be needed in high amounts in the cell, its synthesis has to be tightly regulated as it provides the link between carbon and nitrogen metabolism. For de novo glutamate synthesis 2-oxoglutrate is required. This carbon backbone is derived from the tricarboxylic acid (TCA) cycle, which is a part of the carbon metabolism. Therefore, the need for glutamate and the energy state of the cell have to be concerted.

From bacteria to archaea, glutamate is also of importance if the cell has to cope with external osmolarity changes. During high external osmolarity the outflow of water is prevented by the influx of potassium ions. Glutamate counteracts as an anion for the positively charged potassium. To mention only a few, some Archaea or Enterobacteria like E. coli are known to accumulate glutamate as a so called compatible solute in response to sudden hypersalinity of the environment (Kempf \& Bremer, 1998; da Costa et al., 1998). In B. subtilis, glutamate is not the main osmoprotectant, but it is needed in high amounts during osmotic stress. B. subtilis accumulates proline in molar concentration as main compatible solute and proline is synthesized with glutamate as precursor (Kempf \& Bremer, 1998).

For food industry, glutamate has to be produced in large scale to serve as an additive in a variety of products. Besides other organisms like Brevibacteria, especially the Gram-positive bacterium Corynebacterium glutamicum is used for L-glutamate 
production, with an output of about 1.5 million tons per year (Sano, 2009; Rehm \& Burkovski, 2010). Glutamate enhances the flavor of certain products e.g. potato chips or sauces. The effect on the human gustative nerve is called "umami" (Japanese) also referred to as savoriness (Kondoh \& Torii, 2008). This term means that glutamate brings the flavor of a savory product such as meat, cheese or sea food to mind, foods that contain a lot of proteins. Nowadays, the application of glutamate is controversial as it is supposed to trigger a variety of symptoms e.g. the "Chinese restaurant syndrome", but this has not been directly supported by clinical data yet (Williams \& Woessner, 2009; Jinap \& Hajeb, 2010).

\subsubsection{Glutamate metabolism in Bacillus subtilis}

Bacteria prefer different nitrogen sources for their cellular processes. E. coli favors the inorganic molecule ammonium as nitrogen source (Reitzer, 2003). In contrast, $B$. subtilis utilizes the organic compound glutamine as the preferred source of nitrogen (Detsch \& Stülke, 2003).

In the absence of glutamine, B. subtilis can also assimilate ammonium that is together with 2-oxoglutarate converted to glutamate (Fisher, 1999). At high external concentrations of ammonium, a small fraction of ammonia is present due to the chemical equilibrium. As an uncharged molecule, ammonia can freely diffuse into the cell. At low ammonia concentration or if the $\mathrm{pH}$ value drops, the positively charged ion ammonium has to be actively taken up by the cell. Under this condition the ammonium transporter NrgA is expressed that allows ammonium uptake (Wray et al., 1994; Detsch $\&$ Stülke, 2003). Furthermore, within the cell ammonium is formed by the degradation of amino acids and nucleotides (Fisher \& Débarbouillé, 2002).

In B. subtilis, glutamate is exclusively synthesized via two reactions in the GOGAT cycle (Deshpande \& Kane, 1980; Fisher 1999). The two reactions in the GOGAT cycle are performed by the glutamine synthetase (GS) and the glutamate synthase [GOGAT (glutamine-oxoglutarate-aminotransferase)] which is a heterodimeric enzyme encoded in the gltAB operon. In the first step of the cycle, ammonium is transferred to glutamate catalyzed by the GS in an ATP-dependent manner (see Fig. 1.1). This reaction leads to the formation of glutamine. In the following reaction, the GOGAT catalyzes the transfer of the $\delta$-amino group of glutamine to 2-oxoglutarate that 
is derived from the TCA cycle. In this reaction, the co-factor NADPH is oxidized and two molecules of glutamate are produced. One molecule of glutamate is needed to balance the cycle and the other one can be used as nitrogen donor for the anabolism of the cell. As described above, the biosynthesis of glutamate consumes 2-oxoglutarate as carbon backbone. That is why glutamate synthesis is a very important link between the carbon and the nitrogen metabolism in the cell.

The opposite reaction, the deamination of glutamate to 2-oxoglutarate and ammonium allows the cell to utilize glutamate as a source of carbon. This reaction is catalyzed by the glutamate dehydrogenase $(\mathrm{GDH})$ that is RocG in B. subtilis (see Fig.1.1). This enzyme is only capable of catalyzing the degradation of glutamate and not of its synthesis (Belitsky \& Sonenshein, 1998). Probably, RocG lacks the ability to perform the reverse reaction in vivo due to its low affinity for ammonium $\left(\mathrm{k}_{\mathrm{m}}\right.$ value for ammonium of $18 \mathrm{mM}$ ).

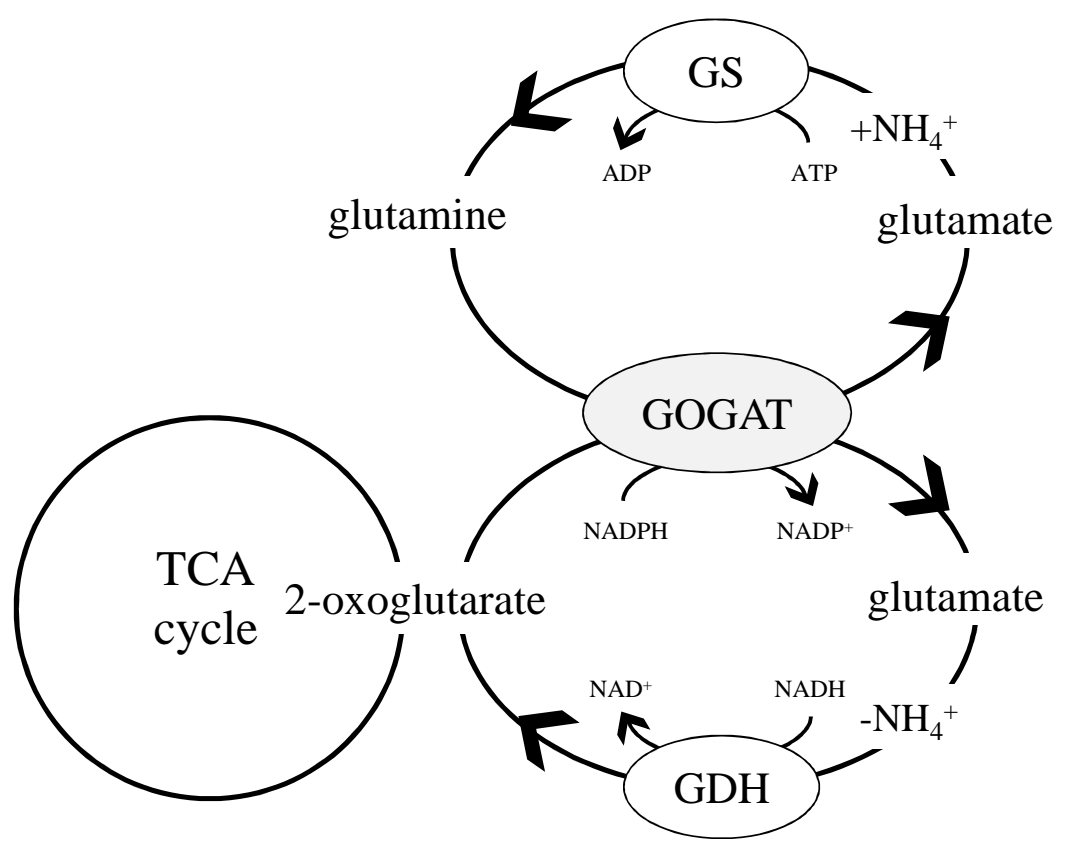

Fig. 1.1 - The GOGAT cycle in B. subtilis.

In $B$. subtilis glutamate is exclusively synthesized by the combined reactions of glutamine synthetase (GS) and the glutamate synthase (GOGAT). In contrast to other organisms, e.g. E. coli or C. glutamicum, the glutamate dehydrogenase $(\mathrm{GDH})$ is only capable of glutamate degradation and not of its synthesis. 
It was shown that the $B$. subtilis GDH can synthesize glutamate with the excess of ammonium in vitro, thus it is hypothesized that the intracellular ammonium concentration in B. subtilis is not sufficient to facilitate this reaction.

In E. coli, the GDH can catalyze the anabolic reaction and forms glutamate in a reductive amination of 2-oxoglutarate with ammonium (Reitzer, 2003). This enzyme exhibits a higher affinity for ammonium than the GDH of B. subtilis $\left(\mathrm{k}_{\mathrm{m}}\right.$ for ammonium of $2.5 \mathrm{mM}$ and $18 \mathrm{mM}$, for E. coli GDH and B. subtilis RocG, respectively; Sharkey \& Engel, 2008; Gunka et al, 2010). Therefore, E. coli GDH is only active at high cellular concentrations of ammonium. Under ammonium excess the GS is poorly expressed and furthermore inhibited by a covalent modification and glutamate is produced by the GDH (Arcondéguy et al., 2001; Reitzer, 2003; Ninfa \& Jiang, 2005). If the ammonium levels drops in the cell, glutamate is synthesized in the GOGAT cycle. Switching between these two pathways, the GOGAT cycle and the GDH reaction, depending on the ammonium supply is very common in microorganisms. Also C. glutamicum can circumvent the energy-consuming reaction of the GS at high ammonium concentrations and forms glutamate catalyzed by the GDH (Rehm \& Burkovski, 2010).

\subsubsection{The regulation of glutamate metabolism in $B$. subtilis}

As mentioned above, the glutamate metabolism is a very important intersection between the carbon and the nitrogen metabolism. In order to assure that the carbon and nitrogen metabolisms are balanced, glutamate synthesis has to be tightly regulated.

In $B$. subtilis, this regulation is achieved by a complex network including the action of transcriptional regulators, protein-protein interactions and the impact of metabolites on enzyme activities and protein complex formation.

The GS is encoded in the $g \ln A$ gene that is part of the $g \ln A R$ operon (Gardner \& Aronson 1984; Strauch et al., 1988). The expression of the GS is regulated in response to nitrogen availability by the two transcriptional regulators TnrA and GlnR (see Tab. 1.1 for an overview on regulation; Schreier et al., 1989; Wray et al., 1996; Fisher, 1999; Fisher \& Débarbouillé, 2002). 
Tab. 1.1 - Regulation of the key enzymes of glutamate metabolism in B. subtilis.

\begin{tabular}{|c|c|c|}
\hline Target genes & Regulator & Regulation and effectors \\
\hline \multirow[t]{2}{*}{$g \ln A$} & $\mathrm{Gln} R$ & $\begin{array}{l}\text { Negative, in complex with feedback } \\
\text { inhibited GS by glutamine }\end{array}$ \\
\hline & TnrA & $\begin{array}{l}\text { Positive in the absence of glutamine; in the } \\
\text { presence of glutamine inactivated by } \\
\text { interaction with feedback inhibited GS }\end{array}$ \\
\hline \multirow[t]{3}{*}{ gltAB } & TnrA & Activation in the presence of ammoinum \\
\hline & GltC & $\begin{array}{l}\text { Activation by } 2 \text {-oxoglutarate; } \\
\text { inhibition by glutamate }\end{array}$ \\
\hline & RocG & $\begin{array}{l}\text { Inhibition of GltC by protein-protein } \\
\text { interaction in the presence of glutamate }\end{array}$ \\
\hline \multirow[t]{5}{*}{$\operatorname{roc} G$} & CcpA & $\begin{array}{l}\text { Inhibition by CcpA in complex with HPr } \\
\text { phosporylated at serine } 46\end{array}$ \\
\hline & RocR & $\begin{array}{l}\text { Activation in the presence of arginine, } \\
\text { ornithine, citrulline or proline }\end{array}$ \\
\hline & SigL & Activation dependent on RocR \\
\hline & AhrC & Activation, requires arginine \\
\hline & AbrB & Inhibtion \\
\hline
\end{tabular}

TnrA is a global regulator of nitrogen metabolism. During nitrogen limitation, TnrA activates among other genes that are required for the utilization of alternative nitrogen sources, the expression of the $g \ln A$ gene, whereas under nitrogen excess, the GS expression is repressed by GlnR (Wray et al., 1996; Wray et al., 2001). The GS influences both, TnrA dependent activation und the transcriptional repression exerted by GlnR. At high concentration of glutamine, the GS is subject to feedback inhibition (Deuel \& Prusiner, 1974). The feedback inhibited GS prevents TnrA from DNA binding by a protein-protein interaction thus inhibiting its own expression if glutamine is available (Wray et al., 2001). Moreover, the feedback inhibited GS activates the DNA binding activity of GlnR and stabilizes GlnR-DNA interaction which represses the $g \ln A$ expression (Fisher \& Wray, 2008). Due to this regulation, the GS is only expressed at 
high level, if nitrogen is limiting and glutamate has to be synthesized. In this regulation mechanism the GS acts as a trigger enzyme that controls its own synthesis.

The gltAB operon codes for the GOGAT in B. subtilis (Deshpande \& Kane, 1980). The big subunit (168 $\mathrm{kDa})$ encoded in the gltA gene is involved in glutamine deamination. The gltB gene codes for the small subunit $(55 \mathrm{kDa})$ that is responsible for NADPH binding and catalyzes the transfer of the amino group to 2-oxoglutarate (Matsuoka \& Kimura, 1986). The expression of the GOGAT depends on both, the carbon and the nitrogen sources (see Tab. 1.1). If glucose and ammonium are present, the gltAB operon is highly transcribed (see Fig. 1.2A). The expression is low in the presence of a poor carbon source or if glutamate or its precursor arginine, ornithine or proline is available (Bohannon et al., 1985; Wacker et al., 2003; Belitsky \& Sonenshein, 2004). In the absence of ammonium, repression of the gltAB operon is mediated by the global regulator TnrA (Belitsky et al., 2000). Thus, the GOGAT is only expressed, if ammonium is present and can be utilized in the GOGAT cycle. Furthermore, for gltAB expression the activity of the transcriptional regulator GltC is required (Bohannon \& Sonenshein, 1989; Belitsky \& Sonenshein, 1995). The GltC protein is encoded directly upstream of the gltAB operon in divergent orientation, whereas the promoter regions of gltC and gltAB are overlapping (Belitsky et al., 2000). GltC is a member of the LysR transcription regulator family that are characterized by a conserved structure with an N-terminal helix-turn-helix motif, necessary for DNA binding, and a C-terminal co-inducer-binding domain (Bohannon \& Sonenshein, 1989; Maddocks \& Oyston, 2008). In addition to the activation of the gltAB operon, GltC is a negative autoregulator of its own synthesis. The gltCAB locus exhibits three GltC binding boxes referred to as Box I, II and III (Belitsky et al., 1995; Belitsky \& Sonenshein, 1995; Picossi et al., 2007). Binding of GltC to Box I is responsible for the negative autoregulation, whereas binding to Box II affects both the autoregulation as well as the activation of the gltAB transcription. In vitro analyses showed the impact of low-molecular weight effectors on GltC binding. At a high glutamate concentration GltC binds to the Boxes I and III leading to the repression of the GOGAT expression (see Fig. 1.2B). In the presence of 2-oxoglutarate, GltC binds to Box I and Box II resulting in a high transcription level of the gltAB operon (Picossi et al., 2007). This regulation by low-molecular weight effectors might contribute to a coordinated expression of the GOGAT in dependence of glutamate availability. 
A

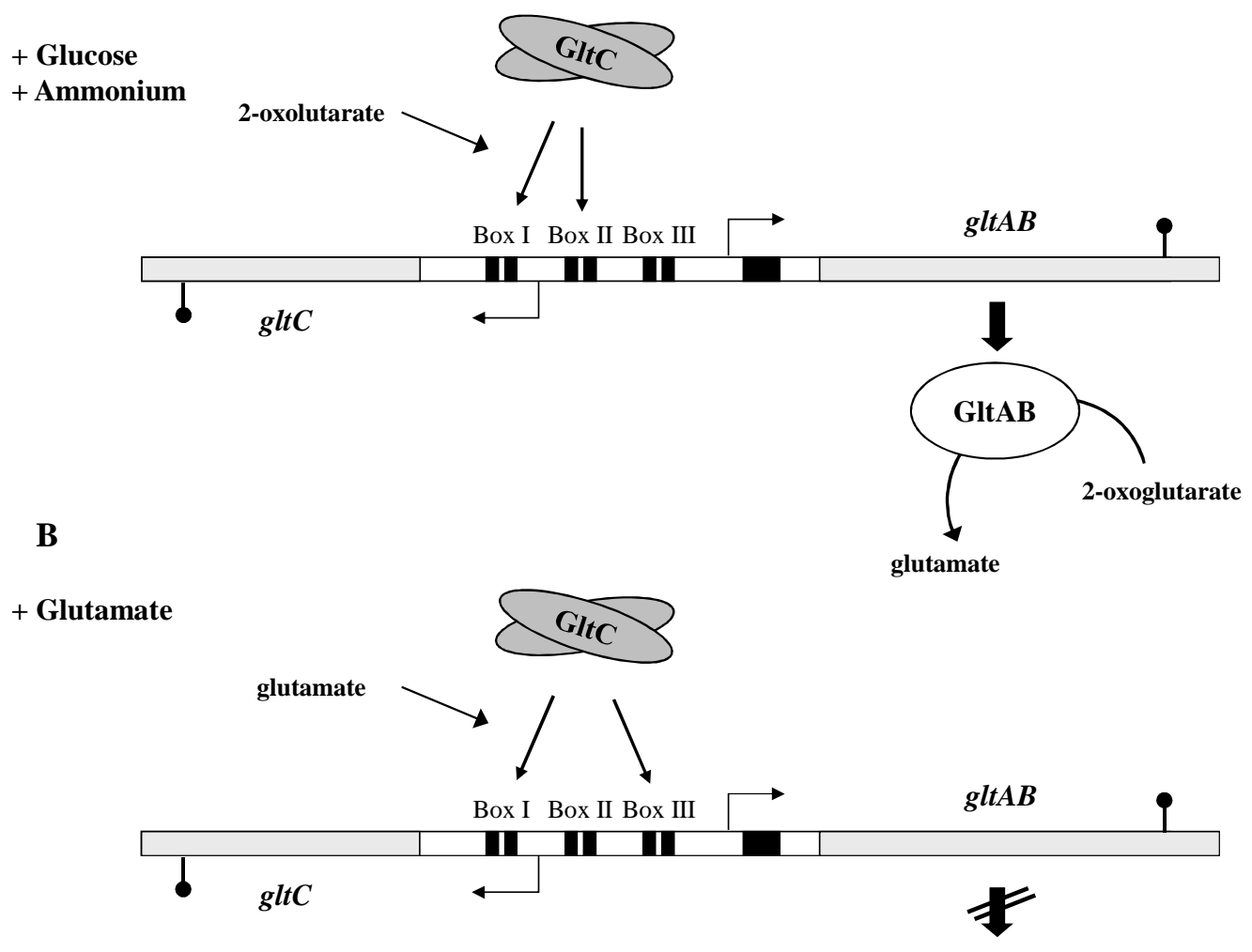

C

+ Arginine
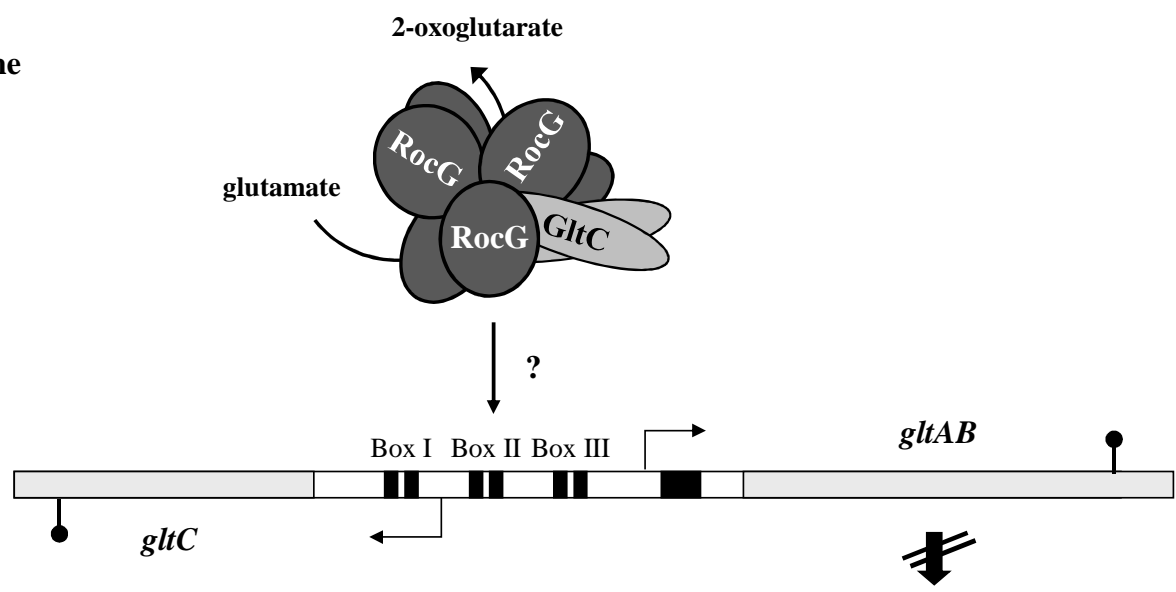

Fig. 1.2 - GOGAT expression in response to the carbon and nitrogen source.

(A) In the presence of glucose and ammonium GltC binds to the Boxes I and II which results in GOGAT expression. Moreover, TnrA can induce the transcription of the $g l t A B$ operon. (B) In the absence of glucose and the presence of glutamate, GltC binds to the Boxes I and III leading to an inhibition of the transcription of the gltAB operon. (C) In the presence of Arginine RocG is expressed and prevents GltC by protein-protein interaction from the activation of the GOGAT expression. 
The expression pattern of the GOGAT and the GDH RocG is mutually exclusive. This is physiologically important in order to avoid a futile cycle of glutamate production and subsequent glutamate degradation. Furthermore, the GOGAT is constitutively expressed in a rocG mutant strain implying that the RocG protein prevents gltAB transcription (Wacker et al., 2003; Commichau et al., 2007b). By an in vivo crosslinking approach it was demonstrated that the RocG protein directly interacts with GltC thus inhibiting the capacity of GltC to activate gltAB transcription (Commichau et al., 2007a). The inhibition of GltC by RocG seems to be furthermore dependent on the presence of the low-molecular weight effector glutamate (Gunka et al., 2010). It has not been analyzed yet, how RocG inhibits the binding capacity of GltC to the promoter regions.

The expression of the $\operatorname{roc} G$ gene coding for the catabolically active glutamate dehydrogenase is also subject to a complex regulation exerted by both the carbon and the nitrogen sources (see Tab. 1.1). The $\operatorname{roc} G$ gene is induced by arginine, ornithine, citrulline or proline (Belitsky \& Sonenshein, 1998). Therefore, B. subtilis can utilize arginine as the sole source of carbon. Arginine is degraded in several reactions to glutamate that can be consumed by RocG to form 2-oxoglutarate (Calogero et al., 1994). The transcription of the $\operatorname{roc} G$ gene depends on the alternative sigma factor $\mathrm{L}$ (SigL) as well as the two transcription regulators RocR and AhrC (Débarbouillé et al., 1991; Calogero et al., 1994; Gardan et al., 1995). Besides the rocG gene the two operons rocABC and rocDEF are part of the RocR regulon that is responsible for the uptake and utilization of arginine. The RocR protein acts together with SigL in the activation of RocG expression (Belitsky \& Sonenshein, 1999; Ali et al., 2003). The inducers of RocR were identified to be ornithine or citrulline (Gardan et al., 1997). $\mathrm{AhrC}$ is a global regulator of the arginine metabolism that induces the roc $A B C$ and rocDEF genes for arginine utilization but represses the gene products for arginine synthesis (Klingel et al., 1995; Miller et al., 1997). It was shown that also the rocG gene is positively regulated by $\mathrm{AhrC}$ but the binding site of $\mathrm{AhrC}$ to the promoter region has not been analyzed yet (Commichau et al., 2007b). Recently, the rocG gene was identified as a target of AbrB that is a repressor that is active during exponential growth phase (Chumsakul et al., 2010).

In addition to this complex regulation exerted by nitrogen sources the $\operatorname{roc} G$ gene is subject to carbon catabolite repression (Belitsky \& Sonenshein, 2004). In the 
presence of a preferred carbon source e.g. glucose, the CcpA protein (catabolite control protein A) interacts with the HPr protein phosphorylated at a serine 46 residue. This complex represses the transcription of the $\operatorname{roc} G$ gene (Belitsky et al., 2004).

As mentioned above, the RocG protein is involved itself in the complex regulation of the glutamate metabolism in B. subtilis. Additionally to its function as the glutamate dehydrogenase it controls GltC activity in the presence of glutamate or a precursor of it (see Fig. 1.2C) (Commichau et al., 2007a). Therefore, RocG is classified as a trigger enzyme that controls gene expression in response to the nitrogen level of the cell to avoid a futile cycle of glutamate synthase and degradation (Commichau \& Stülke, 2008).

In addition to the $\operatorname{roc} G$ gene, $B$. subtilis possesses a second gene, gudB, that codes for a glutamate dehydrogenase. Both proteins share an identity of $74 \%$ and were crystallized as homohexamers (Gunka et al., 2010). In the laboratory strain 168, only the product of the $\operatorname{roc} G$ gene is enzymatically active (Belitsky \& Sonenshein, 1998). The $\operatorname{gudB}$ gene is characterized by a direct duplication of nine base pairs in the open reading frame. This direct repeat of nine base pair leads to a direct duplication of three amino acids directly located in the active site of the enzyme, which results in an inactive protein. In contrast to the RocG expression that is highly regulated, the gudB gene is constitutively transcribed at a high level (Belitsky \& Sonenshein, 1998; Gunka et al., subm.).

The cultivation of a $\operatorname{roc} G$ mutant strain on rich medium or the presence of glutamate as single carbon source provokes the reactivation of the gudB gene (Belitsky \& Sonenshein, 1998; Commichau et al., 2008). The gain of function process is characterized by the precise excision of nine base pairs of the direct repeat leading to the expression of an active glutamate dehydrogenase designated GudB1. The GudB1 protein can fully replace the trigger enzyme RocG. GudB1 is not only able to take over the function of RocG in glutamate degradation but is also capable of controlling GltC activity (Commichau et al., 2007b).

\subsubsection{Impact of glutamate metabolism on phenotypical features of $B$. subtilis}

The enzymes involved in glutamate metabolism are not only important for the appropriate supply with glutamate of the cell during growth. The loss of the GOGAT 
has a severe impact on cell physiology in terms of differentiation processes, emphasizing the pivotal role of glutamate for the cell.

A survival strategy of $B$. subtilis is the formation of biofilms. Biofilms are multicellular communities that are surface associated (López et al., 2010). Wild strain isolates of B. subtilis form biofilms that are highly differentiated in architecture whereas domesticated strains can only form relatively undifferentiated biofilms (Branda et al., 2004).

In the laboratory strain 168 , the loss of the GOGAT results in a strong defect on biofilms formation. This defect cannot be suppressed by the addition of glutamate to the medium, indicating that not the capacity of the GOGAT to produce glutamate is the factor needed for normal biofilms assembly (Chagneau \& Saier, 2004). However, the detailed influence of the GOGAT on biofilms formation has not been characterized yet.

Interestingly, it was shown that the GOGAT affects even more differentiation processes in B. subtilis. Sporulation is a survival strategy that is induced under starvation and high cell density. The dormant cell can overcome nutrient limitation allowing the survival even under the most adverse conditions (Claverys \& Håvarstein, 2007). A GOGAT deficient B. subtilis strain is impaired in sporulation and germination. Moreover, the resistance of the spores in a GOGAT mutant strain is reduced (Ruzal \& Sanchez-Rivas, 2003). The addition of glutamate restores the spore resistance partially in a GOGAT mutant strain but the outgrow of the spores is still strongly impaired (Ruzal \& Sanchez-Rivas, 2003). Spores contain small acid soluble proteins (SASP) in high abundance. These SASPs mainly contribute to spore resistance to UV, heat and osmotic strength (Hackett \& Setlow, 1988). The defect of a gltAB mutant in spore resistance properties linked to SASPs implies that the GOGAT activity is very important for the biosynthesis of SASPs (Ruzal \& Sanchez-Rivas, 2003). Moreover, the GOGAT is needed for the return to a vegetative lifestyle of B. subtilis (Ruzal \& Sanchez-Rivas, 2003).

The loss of the GDH RocG results in a severe growth defect of B. subtilis on rich medium (see Fig. 1.3). This is probably caused by the accumulation of toxic metabolites that are formed during the degradation of arginine (Belitsky \& Sonenshein, 1998). This growth defect is suppressed by the activation of the cryptic glutamate dehydrogenase gene gudB (Belitsky \& Sonenshein, 1998, Gunka et al., subm.). 


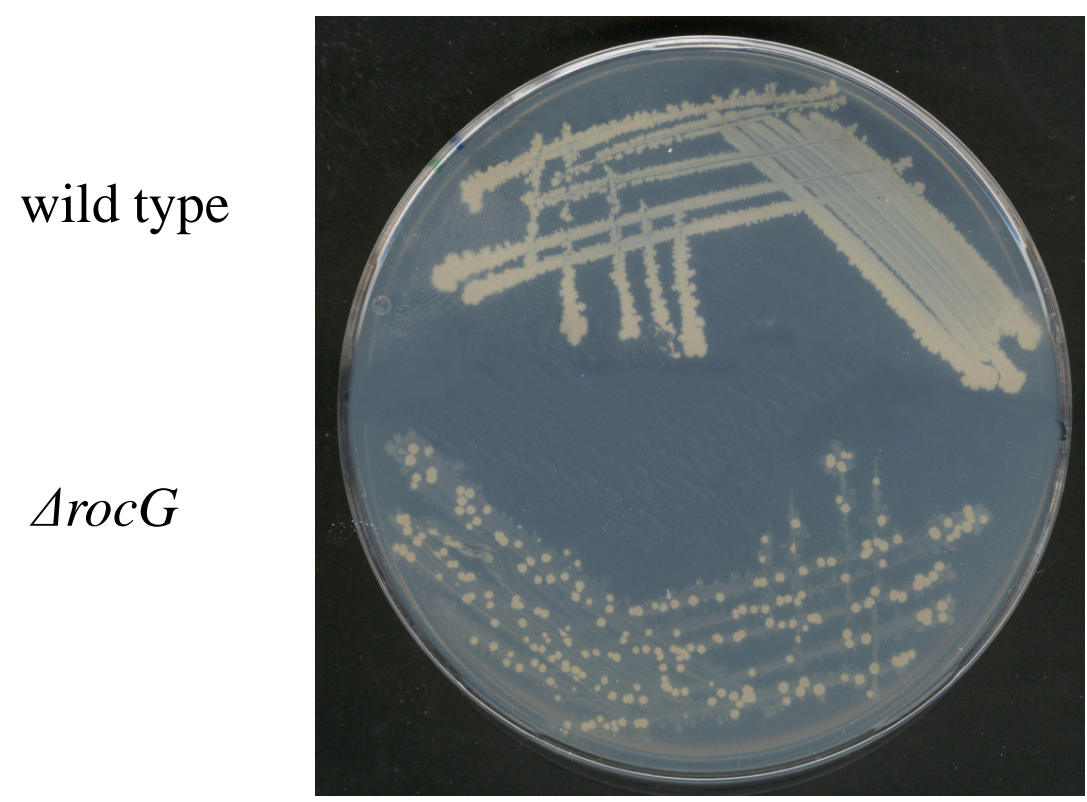

Fig. 1.3 - The growth defect of a $B$. subtilis roc $G$ mutant on rich medium.

B. subtilis wild type grows well on rich medium (upper part of the agar plate). In contrast a $\operatorname{roc} G$ mutant forms translucent colonies and shows an impaired growth (lower part in the background). By the decryptification of the $g u d B$ allele, coding for the second glutamate dehydrogenase, this defect is suppressed. The gudBl mutants grow as well as the wild type (papillae in the lower part).

\subsubsection{The importance of glutamate homeostasis}

The rapid decryptification of the $\operatorname{gudB}$ allele in a $\operatorname{roc} G$ mutant is probably the most extensively studied suppressor mutation observed after perturbating the glutamate homeostasis in B. subtilis. In addition to the $g u d B 1$ mutation a set of other mutations concerning other genes involved in glutamate metabolism were observed in B. subtilis.

A $\operatorname{roc} G \operatorname{gudB}$ double mutant strain that is obviously not able to activate the $\operatorname{gudB}$ gene upon cultivation on rich medium accumulates other mutations that restore growth. A mutant was isolated that had acquired a frameshift mutation in the gltB gene resulting in the expression of a truncated protein and glutamate auxotrophy of this strain (Commichau et al., 2008). The loss of a functional GOGAT seems to balance the glutamate homeostasis of a GDH deficient strain on rich medium. Additionally to mutations affecting the catabolic part of glutamate metabolism, also mutation events occur concerning the anabolic branch of glutamate metabolism in B. subtilis. A mutant defective in the transcription activator GltC is auxotrophic for glutamate, as the 
expression of the GOGAT cannot be induced. However, mutants were found that show an elevated expression of gltA in the absence of the GltC protein. These mutants had acquired a gain of function mutation in the LysR-type regulator GltR (Belitsky \& Sonenshein, 1997). This mutation allows GltR to replace GltC in the activation of gltAB transcription.

As described above, a $\operatorname{roc} G \operatorname{gudB}$ suppressor mutant was isolated that had accumulated a frameshift mutation namely a two base pair deletion in the gltB gene. The cultivation of this glutamate auxotrophic mutant on minimal medium with ammonium as nitrogen source resulted in the reactivation of a functional gltB gene by a third base pair deletion that restores the gltB reading frame (Commichau et al., 2008). These examples clearly demonstrate the robustness of glutamate metabolism according to the availability of the nitrogen source.

Not only in B. subtilis but also in Enterobacteria, suppressor mutations balancing the glutamate homeostasis are known. In 2007, Yan carried out an intensive study on the maintenance of the glutamate pool in Salmonella typhimurium and E. coli. By using mutant strains defective in the GOGAT enzyme a series of suppressor mutants were isolated. In the absence of the GOGAT the glutamine produced by the GS cannot be converted to glutamate. In this strain glutamate can only be synthesized by the GDH, which has a lower affinity for ammonium compared to the GS. This results in an increase of the cellular glutamine and a drastic depletion of the glutamate pool of the cell. The disproportion is balanced by several suppressor mutations. One kind of suppressor mutation affected the GDH activity. For one mutant it was shown that the mutation was located in the $g d h A$ promoter region leading to a high level of GDH expression. Another mutation affected the $g \ln A$ gene and reduced the activity of the GS. Both suppressor mutations compensate the absence of the GOGAT and restore the glutamate/glutamine ratio to wild type level (Yan, 2007). 


\subsection{The controversy of adaptive mutations}

In order to maintain genomic stability, deleterious mutations have to be avoided by the cell. Mutations are changes in the nucleotide sequence including base pair substitutions, deletions or insertions. Nevertheless, without the occurrence of beneficial mutations the process of evolution would not have been possible. Natural selection as a driving force in evolution was first described by Darwin in 1859 (Darwin, 1859). The classic experiment of Luria and Delbrück, the fluctuation test, showed that mutations are even spontaneously acquired without selection pressure (Luria \& Delbrück, 1943). They incubated bacteria cells over a certain period and plated the culture on media treated with bacteriophages. If the presence of the phages would induce mutations in terms of resistance of the bacteria, it would be expected to find almost the same number of resistant bacteria on each plate. Strikingly, the number of resistant bacteria varied extremely on each plate. This experimental setup demonstrated that mutations occur permanently. Before the contact with the phages, the bacteria in the cultures have already randomly acquired mutations that led to resistance. If the mutation event occurs early in the culture, resistant bacteria are highly representative, whereas a late mutation results in only a small number of resistant bacteria. Based on this experiment Lea and Coulson derived a distribution, named Luria-Delbrïck distribution that considers the observed variance and allows the determination of the mutation rate (Lea \& Coulson, 1949).

However, experiments with E. coli lac mutants challenged the model proposed by Luria and Delbrück (Cairns et al., 1988). In contrast to Luria and Delbrück who applied lethal selection, the Cairns system imposes nonlethal stress on cells. An E. coli strain deficient in the lacZ gene cannot utilize lactose. E. coli was shown to possess a cryptic gene that is capable of the hydrolysis of lactose upon decryptification. The experiment demonstrated that activation of this cryptic gene occurs much faster in the presence of lactose than in its absence. Based on this result, it is tempting to speculate, that bacteria might control which mutation occurs in response to selective pressure (Cairns et al., 1988). In 1991, Cairns and Foster modified this system and used an E. coli strain that is chromosomally lacZ negative but harbors a plasmid which encodes a lacZ gene with a frameshift mutation. The gene product exhibits a remaining hydrolysis activity of $2 \%$ of the wild type enzyme and allows poor growth of lactose. Under unrestricted growth, a gain of function mutation occurred at a rate of $10^{-8}$ whereas with lactose as carbon 
source the mutation rate increased hundred fold (Cairns \& Foster 1991; Roth et al., 2006). This experiment suggests the generation of adaptive mutations during selection and argues the statement given by Luria and Delbrïck.

\subsubsection{The frequency of mutations}

The impact of mutations on the organism can be extremely different. The mutation can be deleterious, silent or can even increase the fitness. In E. coli, the chance of acquiring a beneficial mutation is about $10^{-9}$, whereas the rate of deleterious mutations is in range of $10^{-4}$ to $10^{-5}$ (Denamur \& Matic, 2006). Most of the deleterious mutations are purged from the population whereas beneficial mutations increase the overall fitness (Imhof \& Schlötterer, 2001). Studies on E. coli revealed a mutation rate in the range of $10^{-7}$ to $10^{-8}$ during replication (Kunkel et al, 2004). Most of the errors are eliminated by the DNA mismatch repair system (MMR) and are repaired in favor of the base on the template strand. The MMR is evolutionarily highly conserved. The process involves recognition of the mismatch, incision, nucleotide removal and strand resynthesis (Polosina \& Cupples, 2010). In E. coli, recognition of mismatches in the hemimethylated DNA is carried out by the MutS protein. Subsequently, the MutL protein binds to the MutS:DNA complex in an ATP-dependent manner but without ATP hydrolysis. The UvrD helicase is recruited to separate the strands. By complex formation with MutSL the endonucleolytic activity of the MutH protein is activated and MutH excises the mismatch (Modrich \& Lahue, 1996). The resulting gap is filled in by DNA polymerase III, and the DNA ligase carries out the ligation (Polosina \& Cupples, 2010). B. subtilis possesses a functional equivalent of the E. coli MMR system with some striking differences. B. subtilis lacks MutH and UvrD homologues and discrimination between the parental and the newly synthesized strand is not mediated by the methylation state but by the presence of single-strand breaks (Rossolillo \& Albertini, 2001).

Another cellular pathway, transcription, turned out to be important for the emergence of mutations. 


\subsection{Transcription-associated DNA repair}

Besides DNA replication, the process of transcription is important for mutagenesis. During transcription, the nontranscribed strand is subject to mutations. The phenomenon is called transcription-associated mutagenesis. When the RNA polymerase forms a complex with the transcribed strand, the nascent nontranscribed strand is not protected by base pairing anymore and becomes vulnerable to mutagens. The mutations that arise are not expressed until replication (Kim et al., 2010). A study on E. coli suggests that actively transcribed genes acquire more cytosine to thymine mutations due to deamination in the nontranscribed than in the transcribed strand (Beletskii \& Bhagwat, 1996). Moreover, an increase in mutations that changed a TGA stop codon in the non-transcribed strand to a sense codon was observed at high transcription (Klapacz \& Bhagwat, 2005).

Another mechanism that is supposed to generate mutations during transcription is the transcription-coupled nucleotide excision repair (TCR) that acts selectively on the transcribed strand. TCR is a subpathway of nucleotide excision repair (NER) in which DNA lesions are recognized that interfere with the proceeding of the RNA polymerase in a transcribed gene (Hanawalt \& Spivak, 2008). There is evidence that the mechanism of TCR is widespread among bacteria and eukaryotes but it has not been demonstrated in archaea yet (Eisen \& Hanawalt, 1999; Fousteri \& Mullenders, 2008). The process of TCR was first described for eukaryotes by demonstrating that pyrimidine dimers were preferentially removed by nucleotide excision repair in transcribed regions (Bohr et al., 1985).

\subsubsection{The role of Mfd in cellular physiology}

The Mfd protein is the driving force in TCR and is highly conserved in microbial genomes (Selby \& Sancar, 1993; 1994). Mfd stands for mutation frequency decline due to its originally identified effect on mutagenesis (Witkin, 1956; 1966). Later on, Mfd was shown to mediate TCR and has been called transcription repair coupling factor (TCRF) that provides the continuation of transcription after DNA lesions (Selby \& Sancar, 1993; 1994; 1995a; 1995b). Mfd recognizes stalled RNA polymerase caused by a DNA lesion. After displacing the RNA polymerase and its transcripts, Mfd recruits the DNA excision repair machinery that resolves the lesion and allows the restart of 
transcription (Roberts \& Park, 2004; Borukhov et al., 2005; Deaconescu et al., 2006). Evidence was provided that Mfd operates with the nucleotide excision repair system (NER). In vitro analysis showed that Mfd stimulates the UvrABC system (Selby \& Sancar, 1993). The UvrABC system cleaves damaged DNA in an ATP-dependent manner. The UvrA protein initially recognizes a DNA lesion and forms an ATPdependent heterodimer with the UvrB protein. The ATP hydrolysis is performed by the ATPase activity of the UvrB protein that is stimulated upon contact with UvrA (Truglio et al., 2006). In the TCR pathway, Mfd is supposed to recruit UvrA to the damaged DNA (see Fig. 1.4). For the recruitment, the N-terminal part of the Mfd protein seems to be necessary as it has a high homology to the region in the UvrB protein that is essential for the UvrA-UvrB interaction (Selby \& Sancar, 1993; Assenmacher et al. 2006). After loading UvrB onto the damaged DNA, UvrA dissociates and UvrB forms a stable complex with the DNA. Subsequently, UvrC binds to the UvrB:DNA complex and initiates the cleavage of the 3' as well as the 5' end of the DNA (Truglio et al., 2006). In E. coli, the UvrD protein, a helicase II, removes the excised nucleotides and displaces UvrC. Then, the gap is filled in by the DNA polymerase I and ligated by a DNA ligase (Sancar, 1994). The genome of $B$ subtilis, encodes also the UvrABC proteins but lacks an UvrD homologue (Smith et al., 2001; 2002). Besides the Mfd protein, other proteins are supposed to modify RNA polymerase activity. The transcription elongation factors GreA ad GreB suppress RNA polymerase arrest. While GreA can only prevent RNA polymerase from pausing, GreB can even reactivate transcription after RNA polymerase arresting at a roadblock (Borukhov et al., 2005). NusA is an essential transcription termination factor and fulfills a variety of roles. NusA can facilitate transcription termination caused by hairpin-structures. Moreover, it is necessary to synchronize the process of transcription and translation by inducing RNA polymerase pausing (Borukhov et al., 2005). With the exception of Mfd, the other transcription factors have not been proven to be involved in the TCR. 


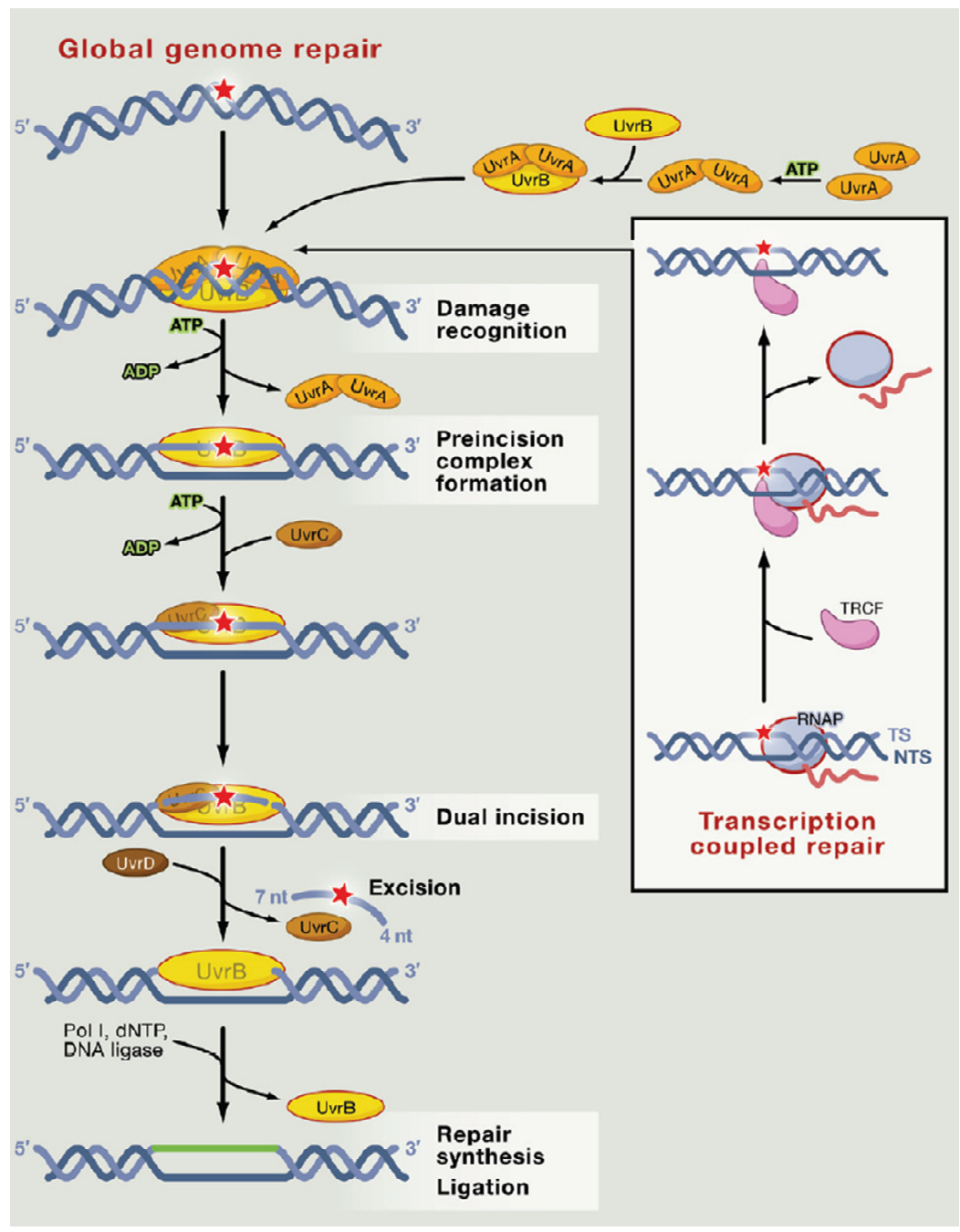

Fig. 1.4 - The role of Mfd in NER (Guo et al., 2010).

The TCRF, Mfd, recognizes a stalled RNA polymerase at a DNA lesion and recruits the NER system to solve the lesion. (The figure is reprinted with kind permission of Elsevier.)

In $B$. subtilis the role of Mfd in mutagenesis was subject to intensive investigations in the last two decades. First evidence for the role of Mfd in TCR was provided by showing that $B$. subtilis Mfd can displace the RNA polymerase in vitro (Ayora et al., 1996). Furthermore, Mfd was shown to be involved in the carbon catabolite repression by displacing RNA polymerase stalled at downstream cre sites (Zalieckas, 1998). Additionally, the role of Mfd in the phenomenon called adaptive or stationary phase mutagenesis in B. subtilis was discovered. The stationary phase mutagenesis means the occurrence of a hypermutable subpopulation, when a nondividing population has to cope with e.g. amino acid starvation pressure or as in the 
Cairns system with carbon end energy starvation (Sung \& Yasbin, 2002). Mfd turned out to be involved in the generation of mutants during amino acid starvation. B. subtilis strains that are auxotrophic for certain amino acids were incubated on medium lacking one of the required amino acids. In a short period of time mutants prototrophic for the previously required amino acid were isolated, indicating the occurrence of stationary phase mutagenesis. A $m f d$ mutant strain was strikingly diminished in its capacity to generate prototrophic revertants in stationary phase implying an important role of the Mfd protein in the process of mutagenesis (Ross et al., 2006). Recently, the importance of transcription in the stationary phase mutagenesis was pointed out in B. subtilis. In this study, a strain that bore a missense mutation in an allele for the synthesis of a particular amino acid was used. Under selection a high number of prototrophic revertants were found, but only if the gene was transcribed (Pybus et al. 2010). This is in agreement with studies on the yeast S. cerevisiae that showed a high frequency of mutations according to high transcription level (Kim et al., 2007). In an isogenic $m f d$ mutant strain not only the transcription level of the respective gene was reduced but moreover, the rate of revertants was significantly decreased (Pybus et al. 2010). This result strongly suggests an important role of Mfd in the stationary phase mutagenesis and the accumulation of adaptive mutations.

Recently, another role of the Mfd protein in E. coli was demonstrated. In vitro analysis showed that Mfd is necessary to clear the conflict between the DNA and the RNA polymerase in a head-on collision. The Mfd protein arranges to solve this conflict in favor of replication by pausing transcription (Pomerantz \& O’Donnell, 2010). 


\subsection{Aims of this work}

In B. subtilis, the glutamate dehydrogenase RocG is a bifunctional trigger enzyme (Commichau et al., 2007a). On the one hand, RocG catalyzes the degradation of glutamate. On the other hand, RocG directly interacts with the transcriptional regulator GltC, thus controlling the ability of GltC to induce the expression of the glutamate synthase. The region in the RocG protein that is necessary for interaction with GltC and the direct mechanism of regulation have not been clarified yet. In order to study the mechanism of RocG-GltC interaction it was intended to isolate mutant RocG proteins that lack the ability to control GltC activity. These mutant proteins would be an appropriate subject for analyzing the mode of interaction between RocG and GltC.

The inactive glutamate dehydrogenase GudB is readily decryptified in a $\operatorname{roc} G$ mutant strain upon growth on complex medium. This suppressor mutation always occurs as the precise deletion of nine base pairs of the direct repeat in the gudB gene and complements the severe growth defect of the $\operatorname{roc} G$ mutant on complex medium. To understand whether this mutation is acquired with an increased rate compared to other mutations, the occurrence of this mutation has to be quantified. In the case of an increased rate it would be of interest to analyze the mechanism and the requirements for the decryptification of the $\operatorname{gudB}$ allele. 


\section{Chapter 2}

Functional dissection of a trigger enzyme: Mutations of the Bacillus subtilis glutamate dehydrogenase RocG that affect differentially its catalytic activity and regulatory properties

The work described in this chapter was published in:

Gunka, K., Newman, J. A., Commichau, F. M., Herzberg, C., Rodrigues, C., Hewitt, L., Lewis, R. J. \& Stülke, J. (2010) Functional dissection of a trigger enzyme: Mutations of the Bacillus subtilis glutamate dehydrogenase RocG that affect differentially its catalytic activity and regulatory properties. J. Mol. Biol. 400: 815-827.

\section{Author contributions:}

This study was designed and interpreted by KG, FMC, RJL and JS. The experiments were done by $\mathrm{KG}$ but FMC and $\mathrm{CH}$ contributed substantially to the generation of the plasmid pools and $\mathrm{CH}$ additionally to the protein purification. The crystallography studies were done by collaboration with JAN, LH, CR and RJL University of Newcastle, Newcastle upon Tyne, UK. KG, FMC, RJL and JS wrote the paper. 


\begin{abstract}
Any signal transduction requires communication between a sensory component and an effector. Some enzymes engage in signal perception and transduction, as well as in catalysis, and these proteins are known as "trigger" enzymes. In this report, we detail the trigger properties of RocG, the glutamate dehydrogenase of Bacillus subtilis. RocG not only deaminates the key metabolite glutamate to form $\alpha$-ketoglutarate but also interacts directly with GltC, a LysR-type transcription factor that regulates glutamate biosynthesis from $\alpha$-ketoglutarate, thus linking the two metabolic pathways.

We have isolated mutants of RocG that separate the two functions. Several mutations resulted in permanent inactivation of GltC as long as a source of glutamate was present. These RocG proteins have lost their ability to catabolize glutamate due to a strongly reduced affinity for glutamate. The second class of mutants is exemplified by the replacement of aspartate residue 122 by asparagine. This mutant protein has retained enzymatic activity but has lost the ability to control the activity of GltC. Crystal structures of glutamate dehydrogenases that permit a molecular explanation of the properties of the various mutants are presented. Specifically, we may propose that D122N replacement affects the surface of RocG. Our data provide evidence for a correlation between the enzymatic activity of RocG and its ability to inactivate GltC, and thus give insights into the mechanism that couples the enzymatic activity of a trigger enzyme to its regulatory function.
\end{abstract}




\section{Introduction}

All signal transduction processes require a component that senses environmental change, and this task can be fulfilled by regulatory proteins that bind directly to specific effector molecules. Signal perception and regulatory output, however, are frequently determined by individual proteins. This mode of signal transduction is arguably best exemplified by two-component signaling systems. By contrast, metabolism is regulated ultimately by the availability of metabolites, and the players that have direct access to this information are the metabolic enzymes. A subset of these enzymes is able to engage in signal transduction and to perform in catalysis, and these are collectively termed “trigger" enzymes (Commichau \& Stülke, 2008).

We are interested in the regulation of nitrogen metabolism in the Gram-positive soil-dwelling bacterium Bacillus subtilis, whose preferred sources of nitrogen are glutamine and ammonium. Ammonium is assimilated by the synthesis of glutamine by glutamine synthetase, encoded by the $g \ln A$ gene. Glutamine is subsequently used for the reductive amination of 2-oxoglutarate by glutamate synthase, encoded by the genes of the gltAB operon (see Fig. 2.1A) (Fisher, 1999). Expression of this operon is controlled by two transcription factors. First, TnrA protein represses the gltAB operon in the absence of ammonium (Wray et al., 1996; Belitsky et al., 2000; Yoshida et al., 2003). Second, the LysR-type regulator GltC activates transcription of the operon in the presence of sugars and in the absence of arginine (Bohannon \& Sonenshein, 1989; Commichau et al., 2007b). The activity of these two transcriptional regulators can be controlled by trigger enzymes. GlnA interacts with TnrA, and the catabolic glutamate dehydrogenase RocG interacts with GltC (Wray et al., 2001; Belitsky \& Sonenshein, 2004; Commichau et al., 2007a; Commichau et al., 2007b) (see Fig. 2.1B). In addition, GltC is modulated by metabolites of the glutamate dehydrogenase reaction: 2oxoglutarate and glutamate stimulate and inhibit the activity of GltC, respectively (Picossi, et al., 2007).

Thus, the information available to the enzymes of glutamine synthesis and glutamate degradation is shared with transcription factors in the modulation of their activities. The use of trigger enzymes in the transcriptional regulation of metabolic genes allows direct feedback between metabolite availability and gene expression, thus 
maintaining a pool of key metabolic intermediates in the cell at a constant concentration.

(A)

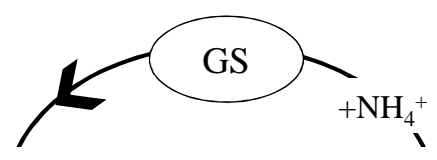

glutamine

glutamate

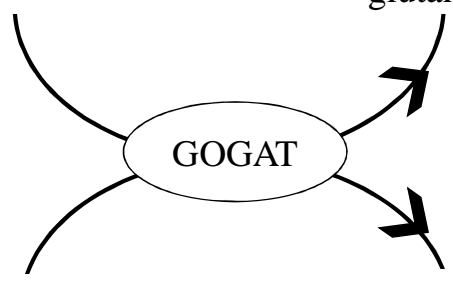

2-oxoglutarate

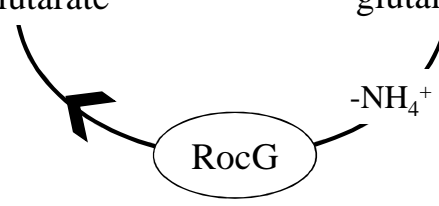

(B)

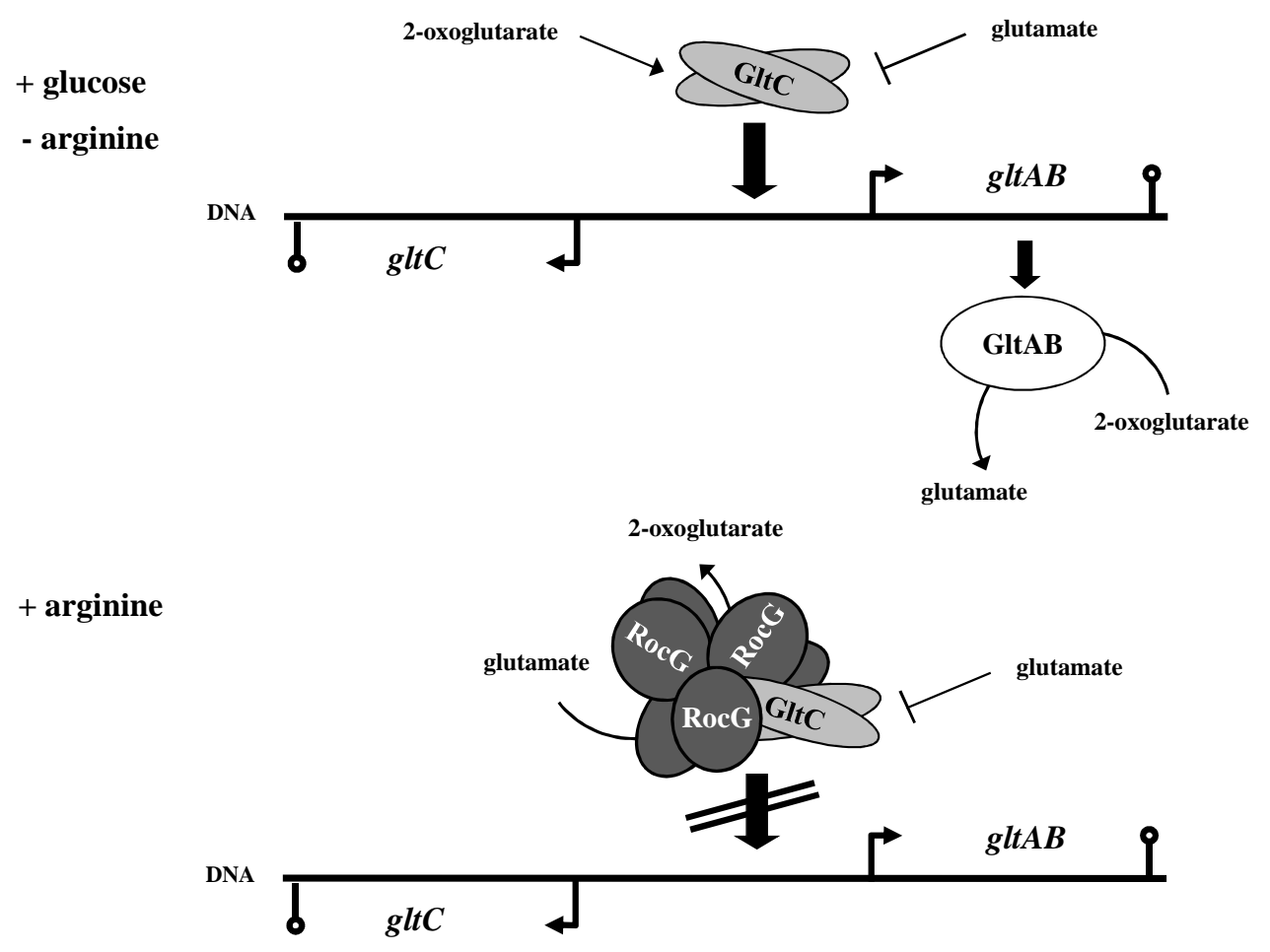

Fig. 2.1 - Glutamate metabolism in B. subtilis and its regulation.

Fig. legend see next page. 


\section{Fig. 2.1 - Glutamate metabolism in B. subtilis and its regulation.}

(A) Glutamate is synthesized in the GOGAT cycle by the combined reactions of glutamine synthetase (GS) and glutamate synthase (GOGAT). The degradation of glutamate to 2-oxoglutarate and ammonium is catalyzed by the strictly catabolic glutamate dehydrogenase RocG. (B) In the absence of arginine, the transcriptional activator GltC (light-gray ovals) stimulates the expression of the gltAB operon. In the presence of arginine, RocG (dark-gray ovals) directly interacts with GltC, leading to inactivation of the transcription factor. Moreover, the activity of GltC is affected by 2-oxoglutarate and glutamate.

Glutamate is one of the major metabolites in any living cell and is either taken up directly from the environment, generated from peptides or amino acids such as arginine and proline, or synthesized from 2-oxoglutarate. In Escherichia coli, glutamate accounts for as much as $40 \%$ of the cellular metabolite pool (Yuan et al., 2009). In the absence of organic nitrogen sources, glutamate is synthesized by glutamate synthase, as summarized above. In many organisms, including E. coli, glutamate dehydrogenase is capable of directly generating glutamate by the amination of 2-oxoglutarate. However, glutamate dehydrogenase has a low affinity for ammonium; therefore, this reaction can take place only at high ammonium concentrations (Yuan et al., 2009). Glutamate synthase is the only enzyme in $B$. subtilis that can produce glutamate; by contrast, roc $G$ encoded glutamate dehydrogenase has an exclusively catabolic activity in this organism (see Fig. 2.1A) (Belitsky \& Sonenshein, 1998).

Glutamate would thus appear to be a particularly important cellular metabolite in B. subtilis and other organisms. For instance, glutamate metabolism is very tightly controlled by at least five different transcription factors: TnrA and GltC control the biosynthetic operon gltAB; the expression of $\operatorname{roc} G$ is subject to carbon catabolite repression exerted by $\mathrm{CcpA}$; and synthesis of $\operatorname{roc} G$ in the presence of arginine is mediated by the transcription activators RocR and AhrC (Klingel et al., 1995; Belitsky \& Sonenshein, 1999; Belitsky et al., 2004). Moreover, both B. subtilis and E. coli respond to any perturbation in glutamate homeostasis with spontaneous suppressor mutations that bring the glutamate pools back into balance (Yan, 2007; Commichau et al., 2008).

Glutamate metabolism in B. subtilis is, unusually, characterized by the presence of a second glutamate dehydrogenase, gudB (Belitsky \& Sonenshein, 1998). The enzyme encoded by this gene is active in wild-type isolates of B. subtilis, but is cryptic in laboratory strain 168 due to an insertion of three amino acids close to the active site of 
the enzyme (Zeigler et al., 2008). Indeed, roc $G$ mutant strains readily recover glutamate homeostasis by decryptification of gudB (Belitsky \& Sonenshein, 1998; Commichau et al., 2008).

Hence, RocG appears to have a dual role in glutamate metabolism. It is involved in glutamate catabolism but, if glutamate is available, interacts with GltC, the transcription activator of the gltAB operon, and this interaction is inhibitory to GltC. However, in the absence of arginine or in the presence of glucose, either of which leads to a reduction in RocG expression, free GltC activates transcription of the gltAB operon, resulting in the biosynthesis of glutamate (Commichau et al., 2007b). Some variants of GltC are constitutively active as a transcription activator, suggesting that these GltC proteins cannot be inactivated by RocG (Belitsky \& Sonenshein, 1995). Indeed, a reduction in the interaction between RocG and one of these GltC variants was previously observed in vivo (Commichau et al., 2007b).

In this study, we have isolated and characterized RocG variants whose ability to control GltC activity is affected. Several amino acid substitutions in RocG cause permanent inactivation of GltC in media containing glutamate or its precursor, arginine. These enzymes are, on the other hand, strongly impaired in catalysis, and the affinity for glutamate is severely reduced. One of the RocG variants that we have isolated has retained its enzymatic activity but has lost the ability to inactivate GltC. We have also determined the crystal structures of both the major glutamate dehydrogenase and the secondary glutamate dehydrogenase from B. subtilis (RocG and GudB1, respectively). These structures have allowed us to analyze the effect of RocG variations at the molecular level and to gain insights into the nature of the interaction between RocG and GltC. 


\section{Materials and Methods}

\section{Bacterial strains and growth conditions}

The E. coli strains DH5a, (Sambrook et al., 1989) XL1-Red (Stratagene, Amsterdam, Netherlands), and BL21/DE3 (pLysS) (Novagen, Darmstadt, Germany) were used for cloning experiments, random mutagenesis, and protein expression, respectively. The B. subtilis strain GP28 (trpC2 rocG::Tn10 spc $\Delta g u d B:$ cat amyE::gltA'-'lacZ aphA3) (Commichau et al., 2007a) was used for the screening and characterization of $\operatorname{roc} G$ mutants. B. subtilis GP27 (trpC2 $\triangle$ gudB::cat amyE::gltA“-' lacZ aphA3) (Commichau et al., 2007a) was used to estimate the level of overexpression of RocG in the screening system. B. subtilis was grown in $\mathrm{C}$ minimal medium containing ammonium as the nitrogen source, and $5 \mathrm{~g} / \mathrm{l}$ glucose (C-Glc medium), $5 \mathrm{~g} / \mathrm{l}$ arginine (CR medium), or $8 \mathrm{~g} / \mathrm{l}$ glutamate (CE medium) as the carbon source (Wacker et al., 2003). CR-Glc medium is C minimal medium containing $5 \mathrm{~g} / 1$ glucose and $5 \mathrm{~g} / \mathrm{l}$ arginine. CS-Glc contains $6 \mathrm{~g} / \mathrm{l}$ sodium succinate and $5 \mathrm{~g} / \mathrm{l}$ glucose. CSE-Glc medium is CS-Glc with $8 \mathrm{~g} / 1$ glutamate. The media were supplemented with tryptophan $(50 \mathrm{mg} / \mathrm{l})$. E. coli was grown in LB medium, and transformants were selected on plates containing ampicillin $(100 \mu \mathrm{g} / \mathrm{ml})$. LB and SP plates were prepared by addition of $17 \mathrm{~g} / \mathrm{l}$ Bacto agar (Difco, Lawrence, USA) to the medium.

\section{DNA manipulation}

Transformation of E. coli was performed using standard procedures (Sambrook et al., 1989). The NucleoSpin Plasmid kit (Macherey and Nagel, Düren, Germany) was used to isolate plasmid DNA from E. coli and B. subtilis. Prior to plasmid purification, B. subtilis cells were incubated with $3 \mathrm{mg} / \mathrm{ml}$ lysozyme (Serva, Heidelberg, Germany) dissolved in buffer 1 of the NucleoSpin Plasmid kit to increase the yield of plasmid DNA recovery. Chromosomal DNA of B. subtilis was isolated as described previously (Kunst \& Rapoport, 1995). Restriction enzymes, T4 DNA ligase, and, for PCR, Phusion ${ }^{\mathrm{TM}}$ DNA polymerase (New England Biolabs, Frankfurt am Main, Germany) were all used as recommended by the manufacturers. DNA fragments were purified using the QIAquick PCR Purification kit (Qiagen, Hilden, Germany), and all DNA sequences were determined using the dideoxy chain termination method (Sambrook et al., 1989). 


\section{Random mutagenesis of rocG}

To study the effect of point mutations in RocG that affect GltC activity, we generated plasmid pGP529, which allows the expression of roc $G$ in $B$. subtilis under the control of the strong $\operatorname{deg} Q 36$ promoter (Martin-Verstraete et al., 1994) as follows: rocG was amplified using the primers IW18 (5'AAAAGGATCCGCGCTTACATTACAG) and IW19 (5'AAACTGCAGTCATTAGACCCATCCGCGGAAACG), and the resulting PCR product was digested with BamHI and PstI before cloning into similarly restricted pBQ200 to yield pGP529. Plasmid pGP529 was subjected to random mutagenesis using the E. coli mutator strain XL1-Red. For this purpose, pGP529 was used to transform E. coli XL1-Red, and the cells were plated on 40 LB plates resulting in approximately 80 colonies per plate. The colonies from each plate were resuspended in $1 \mathrm{ml}$ of LB medium, and $100 \mu \mathrm{l}$ of each suspension was used to inoculate $100-\mathrm{ml}$ flasks containing $10 \mathrm{ml}$ of LB medium. The cultures were grown for $48 \mathrm{~h}$ at $37^{\circ} \mathrm{C}$ to allow the emergence of mutations. Plasmid DNA from each culture was isolated individually and used to transform the indicator strain B. subtilis GP28, which contains a translational gltA-lacZ fusion to monitor the effect of mutant RocG proteins on the activity of GltC. Transformants were selected on SP plates containing $2 \mu \mathrm{g} / \mathrm{ml}$ erythromycin plus $25 \mu \mathrm{g} / \mathrm{ml}$ lincomycin.

\section{Plasmids for the expression of $\operatorname{roc} G$ and $g u d B$ alleles}

Plasmid pGP902 was used for the overexpression of Strep-tagged wild-type RocG (Commichau et al., 2007a). All plasmids used in this study are listed in Tab. S2. For the expression of GudB1, the active variant of B. subtilis GudB, we constructed plasmid pGP864 by cloning a PCR fragment obtained with primers KG20 (5' AAAGAGCTCGATGGCAGCCGATCGAAACACCG) and KG21 (5' TTTGGA TCCTCATTATATCCAGCCTCTAAAACGCGAAGCTT), which have used the chromosomal DNA of the gudB1 mutant strain GP801 (Commichau et al., 2008) as template. For overexpression of Strep-tagged RocG mutant proteins, the plasmids pGP856, pGP857, pGP858, pGP865, and pGP866 were constructed. The corresponding rocG mutant alleles were amplified with the primer pair PT5/PT6, (Commichau et al., 2007a) using plasmids pGP852, pGP853, pGP854, pGP932, and pGP933 as template DNAs (see Tab. 2.1). All PCR products were digested with SacI and BamHI, and 
cloned into the vector pGP172 (Merzbacher et al., 2004) cut with the same enzymes. The identity of the cloned inserts was verified by DNA sequencing.

\section{Protein purification}

E. coli BL21/DE3 (pLysS), transformed by plasmids encoding the appropriate glutamate dehydrogenases, was used as host for the overexpression of recombinant proteins. Cultures were grown in 11 of LB medium at $37^{\circ} \mathrm{C}$; expression was induced by the addition of IPTG (final concentration, $1 \mathrm{mM}$ ) to logarithmically growing cultures $\left(\mathrm{OD}_{600}=0.8\right)$, and the cells were harvested $2 \mathrm{~h}$ after induction. The pellets were resuspended in $30 \mathrm{ml}$ of disruption buffer $(100 \mathrm{mM}$ Tris- $\mathrm{HCl}, 150 \mathrm{mM} \mathrm{NaCl}$, and 10 $\mathrm{mM}$ ethylenediaminetetraacetic acid, $\mathrm{pH}$ 8.0). The cells were disrupted by using a French Press (20,000 psi, 138,000 kPa; Spectronic Instruments, Garforth, UK). The extracts were passed over a 1-ml Strep-Tactin ${ }^{\circledR}$ column (IBA, Göttingen, Germany), and the bound material was washed with $6 \mathrm{ml}$ of disruption buffer before being eluted with $3.5 \mathrm{ml}$ of disruption buffer containing $2.5 \mathrm{mM}$ desthiobiotin (IBA, Göttingen, Germany). The Bio-Rad dye-binding assay was used to determine protein concentrations, with bovine serum albumin as standard.

\section{Western blot analysis}

For Western blot analyses of RocG, B. subtilis crude extracts were separated by SDS-PAGE and transferred to a polyvinylidene difluoride membrane (Bio-Rad, Munich, Germany) by electroblotting. RocG was detected with rabbit polyclonal antiserum raised against B. subtilis RocG (Commichau et al., 2007a). The anti-RocG antibodies were visualized with anti-rabbit IgG-AP secondary antibodies (Chemikon International, Temecula, USA) with the CDP* detection system (Roche Diagnostics, Basel, Switzerland).

\section{Enzyme assays}

The amination reaction catalyzed by RocG was assayed at room temperature by measuring the decrease in $\mathrm{NADH}_{2}$ absorption at $340 \mathrm{~nm}$ (Ultrospec 2100 pro; GE Healthcare, Munich, Germany) under the following conditions: $10 \mathrm{mM}$ 2-oxoglutarate, $0.1 \mathrm{mM} \mathrm{NADH}_{2}$, and $0.1 \mathrm{M}$ ammonium chloride in $100 \mathrm{mM}$ Tris- $\mathrm{HCl}$ buffer ( $\mathrm{pH} 7.3$ ). 
The deamination reaction was assayed by measuring the increase in $\mathrm{NADH}_{2}$ absorption at $340 \mathrm{~nm}$ in a mixture of $0.1 \mathrm{mM} \mathrm{NAD}^{+}$and $20 \mathrm{mM}$ monosodium L-glutamate in 50 $\mathrm{mM}$ potassium phosphate buffer $\left(\mathrm{pH}\right.$ 7.7). For the determination of $\mathrm{K}_{\mathrm{m}}$ values, a wide concentration range of the relevant substrates (glutamate and ammonium for catabolic and anabolic reactions, respectively) was used.

\section{Crystallization and structure determination}

RocG(E93K) and GudB1 proteins were purified as described above, with the exception of an additional purification step of gel filtration using a Superdex 200 column (GE Healthcare, Buckinghamshire, UK) and with the elution profile of both proteins being consistent with a hexameric assembly. The proteins were buffer exchanged into $10 \mathrm{mM}$ Tris- $\mathrm{HCl}(\mathrm{pH} 8.0)$ and $100 \mathrm{mM} \mathrm{NaCl}$, and concentrated for crystallization at $10 \mathrm{mg} / \mathrm{ml}$ using a Millipore 30-kDa cutoff centrifugal concentrator. RocG(E93K) crystals were grown by hanging-drop vapor diffusion using a mother liquor of 20\% (wt/vol) PEG 3350 and 20\% (vol/vol) glycerol. Single crystals were loop mounted and flash cooled in liquid nitrogen. GudB1 crystals were grown from solutions containing 20\% (wt/vol) PEG 3350 and 0.15 M sodium malonate. Crystals were transferred for approximately $1 \mathrm{~min}$ to a cryoprotectant of crystallization buffer supplemented with $25 \%$ (vol/vol) glycerol before being loop mounted and flash cooled in liquid nitrogen. Data collection for both RocG(E93K) and GudB1 was performed on beamline I02 at the Diamond light source, with diffraction extending to maximum resolutions of 2.3 and $2.4 \AA$, respectively. Data sets were processed using XDS (Leslie, 1992) then scaled and merged using SCALA (Evans, 1993) in the CCP4 suite of programs (CCP4, 1994). Both structures were solved by molecular replacement using the program MOLREP (Vagin \& Teplyakov, 1997) and the Pyrococcus furiosus glutamate dehydrogenase (1GTM) as search model (Yip et al., 1995). Manual model building in Coot (Emsley \& Cowtan, 2004) was interspersed with rounds of refinement until convergence. The RocG(E93K) structure was refined using the program REFMAC (Murshudov et al., 1997) to $R$-factor/ $R_{\text {free }}$ values of $0.19 / 0.24$, whereas GudB1 was refined with PHENIX REFINE (Adams et al., 2002) to $R$-factor/ $R_{\text {free }}$ values of $0.25 / 0.28$. 


\section{Results}

\section{Isolation of rocG mutant alleles}

Plasmid pGP529 carrying the $\operatorname{roc} G$ gene under the control of a strong constitutive promoter was subjected to random mutagenesis using the E. coli mutator strain XL1Red, and the plasmid pools obtained were used to transform the indicator strain B. subtilis GP28 as described in Materials and Methods (Commichau et al., 2007a). To identify $\operatorname{roc} G$ mutant alleles, we transferred the transformants onto $\mathrm{CR}$ and CR-Glc minimal media containing $40 \mu \mathrm{g} / \mathrm{ml}$ 5-bromo-4-chloro-3-indolyl- $\beta$-D-galactopyranoside (X-Gal). Cells expressing wild-type RocG form pale blue colonies on CRGlc-X-Gal plates. In our system, RocG is constitutively expressed under the control of a glucose-insensitive promoter. The RocG amounts present in the cell in this system were about 20-fold increased as compared to a wild-type strain expressing $\operatorname{roc} G$ from its own promoter (B. subtilis GP27; data not shown). The arginine present in the medium can be converted into glutamate, the substrate of the glutamate dehydrogenase RocG. The catalytically active RocG inhibits GltC, thus reducing the expression of gltA-lacZ fusion. A reasonable explanation for the residual activity of GltC is that glucose is converted into 2-oxoglutarate, which is a positive effector of GltC (Picossi et al., 2007). As a result, GltC cannot be fully inhibited by RocG if the bacteria grow on CR-Glc-XGal plates due to sources of $\alpha$-ketoglutarate. By contrast, wild-type RocG completely inhibits GltC activity on CR-X-Gal plates, resulting in the formation of white colonies. In the absence of glucose, no $\alpha$-ketoglutarate is formed by the tricarboxylic acid cycle, and GltC cannot be stimulated by 2-oxoglutarate and cannot activate the gltA-lacZ fusion.

CR-Glc-X-Gal medium was used to screen for RocG variants that permanently inhibited GltC, resulting in white colonies. About 60 white colonies were obtained, and permanent inhibition of GltC due to the $\operatorname{roc} G$ mutant allele was verified by retransformation for 10 candidates. These plasmidborne $\operatorname{roc} G$ alleles encode so-called "superrepressor" variants of RocG.

Since catalytically competent glutamate dehydrogenase is required for B. subtilis to utilize arginine as carbon and nitrogen source, we used CR-X-Gal plates to isolate monofunctional RocG variants that are catalytically active but are unable to inhibit GltC. Strain GP28 expressing wild-type RocG forms white colonies on CR-X-Gal 
plates due to the complete inactivation of GltC by RocG. By contrast, cells expressing monofunctional RocG proteins were expected to form dark-blue colonies on CR-X-Gal plates. This expectation was further supported by an in silico analysis of the regulatory network of glutamate metabolism, which highlighted the possibility of using CR-X-Gal medium to screen for monofunctional RocG variants. Indeed, two darkblue colonies on CR-X-Gal plates were obtained after the transformation of B. subtilis GP28 with the pool of mutant plasmids. The plasmids of these two candidates were isolated and used to retransform B. subtilis GP28. These transformants were able to grow with arginine as the sole carbon and nitrogen source, and formed dark-blue colonies on CR-X-Gal plates. Thus, these $\operatorname{roc} G$ alleles encode active glutamate dehydrogenases that appear to have lost the ability to inhibit the transcription activator GltC.

Mutations of the superrepressor and monofunctional $\operatorname{roc} G$ alleles were identified by sequencing. In all cases, changes in RocG properties were caused by single base-pair exchanges resulting in single amino acid substitutions. Superrepressor mutations are scattered throughout the entire $\operatorname{roc} G$ sequence, whereas the two monofunctional $\operatorname{roc} G$ alleles carried the same mutation. The position of the mutations in the amino acid sequence of RocG is shown schematically in Fig. 2.2, and the plasmids containing the $\operatorname{roc} G$ mutant alleles and the corresponding mutations are listed in Tab. 2.1.

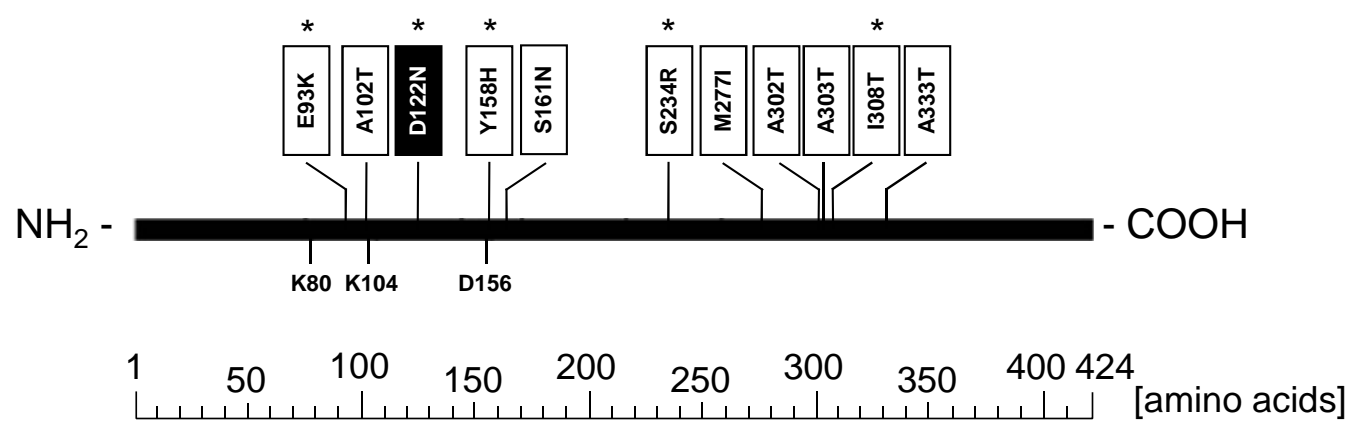

Fig. 2.2 - Mutations found in RocG that affect the regulation of GItC activity by RocG.

Amino acid exchanges leading to a superrepressor phenotype are shown in empty boxes. The mutation of the monofunctional RocG variant is depicted by a filled box. RocG mutant variants that were further analyzed are indicated by asterisks. Amino acids involved in glutamate binding at the active site of RocG are indicated below the primary sequence. 
Tab. 2.1 - Mutations affecting the inhibition of GItC by RocG

\begin{tabular}{ccccc}
\hline Plasmid & Allele & Mutation & $\begin{array}{c}\text { Amino acid } \\
\text { substitution }\end{array}$ & $\begin{array}{c}\text { Corresponding } \\
\text { expression vector }\end{array}$ \\
\hline pGP852 & rocG-SR1 & T923C & I308T & pGP856 \\
pGP853 & rocG-SR2 & C702A & S234R & pGP857 \\
pGP854 & rocG-SR3 & G277A & E93K & pGP858 \\
pGP855 & rocG-SR4 & G997A & A333T & \\
pGP933 & rocG-SR5 & T472C & Y158H & pGP866 \\
pGP867 & rocG-SR6 & G907A & A303T & \\
pGP868 & rocG-SR7 & G904A & A302T & \\
pGP869 & rocG-SR8 & A304G & A102T & \\
pGP870 & rocG-SR9 & A479G & S161N & \\
pGP871 & rocG-SR10 & G831A & M277I & \\
pGP932 & rocG-MF & G364A & D122N & pGP865 \\
\hline
\end{tabular}

For further analyses, we selected four superrepressor mutants and one monofunctional RocG variant. To exclude the possibility that the observed effects result from altered cellular levels of the mutant RocG proteins in comparison to the wild-type protein, we determined the RocG concentration by Western blot analysis. Strains carrying plasmid pGP529 (wild-type RocG) and pBQ200 (empty vector) were used as controls. The strain carrying pBQ200 did not synthesize any protein that was recognized by the anti-RocG antibodies, indicating that any immunodetection is specific for RocG (Fig. 3.2). The cellular concentrations of all RocG variants were similar to that of the wild-type protein (see Fig. 2.3). Thus, the $\operatorname{roc} G$ phenotypes are directly attributable to changes in the biochemical properties of the mutant enzymes. 


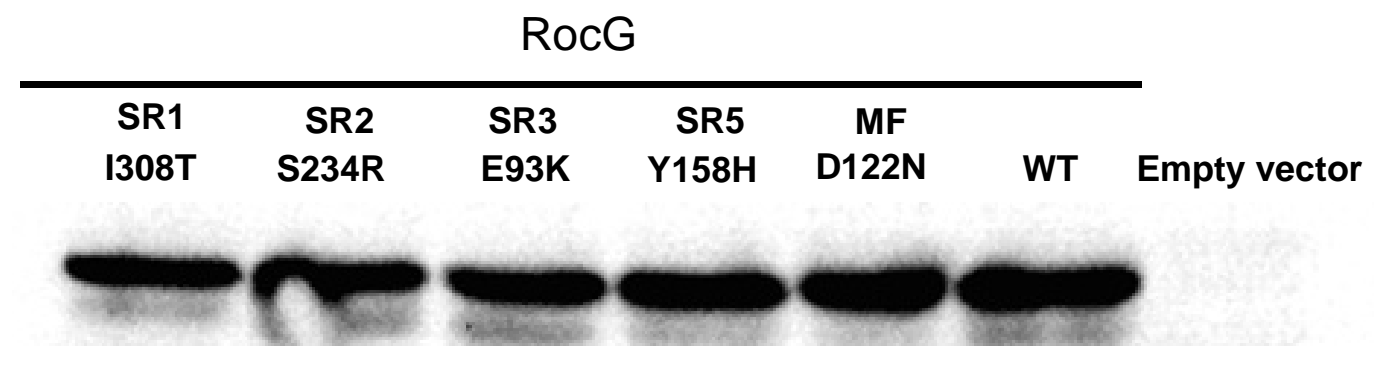

Fig. 2.3 - Expression of RocG mutant proteins.

Crude extracts were isolated from B. subtilis strain GP28 (rocG $\operatorname{coudB}$ ) expressing the RocG superrepressor (SR) proteins SR1, SR2, SR3, and SR5, and the monofunctional (MF) RocG protein grown in CSE minimal medium. Strain GP28 either expressing the RocG wild-type (WT) protein or carrying the empty vector pBQ200 served as control. The proteins were detected using rabbit polyclonal antibodies raised against $B$. subtilis RocG. Samples (15 $\mu \mathrm{g})$ of crude extract were applied per lane.

\section{Impact of RocG mutant proteins on GltC activity}

The impact of RocG mutations on the activity of GltC was quantified after the growth of B. subtilis GP28 carrying relevant plasmids in minimal media. As shown in Tab. 2.2, B. subtilis GP28 carrying the empty vector pBQ200 exhibited constitutive gltA expression because of the absence of a functional glutamate dehydrogenase to inhibit GltC. The strain carrying pGP529, encoding wild-type rocG, showed little promoter activity on media containing arginine. By contrast, gltA-lacZ activity was high in the absence of arginine (presumably reflecting the activity of GltC). This suggests that the inhibition of GltC by RocG did not occur under these conditions. This is in good agreement with previous reports indicating that RocG does not inhibit GltC activity in the absence of arginine even if $\operatorname{roc} G$ is overexpressed. Moreover, the effect of arginine cannot be due to any effect of RocG expression, but some other role of arginine, such as acting as a source of glutamate, must be involved (Belistky \& Sonenshein, 2004; Commichau et al., 2007b).

The plasmids encoding the superrepressor roc $G$ alleles all caused very low GltC activity in CR medium containing glucose. These RocG variants did not support growth with arginine as the single carbon source, suggesting that their enzymatic activity was affected by the mutations (see the text below). The GltC activity of the strains carrying the superrepressor RocG variants was very low in CSE medium containing glucose. By contrast, these RocG variants had only a minor inhibitory impact on GltC activity in a 
medium that did not contain glutamate, or a source of it (i.e., CS medium containing glucose). This observation suggests that the presence of glutamate facilitates the inhibition of GltC by RocG. Moreover, the mutant RocG proteins are still capable of responding to the presence of glutamate. The monofunctional RocG protein did not inhibit GltC activity under any condition tested in this study, and the B. subtilis strain expressing this mutant protein was able to utilize arginine as the single carbon source, suggesting that RocG-D122N was enzymatically active in glutamate degradation. Thus, the D122N mutation appears to have uncoupled the two functions of RocG and converted it from a trigger enzyme into a conventional enzyme with just a metabolic role.

Tab. 2.2 - Effect of the different RocG variants on GltC activity

\begin{tabular}{lccccc}
\hline Plasmid & RocG variant ${ }^{\mathrm{a}}$ & & \multicolumn{2}{c}{ gltA-lacZ expression $^{\mathrm{b}}$} & \\
& & $\mathrm{CR}$ & CR-Glc & CS-Glc & CSE-Glc \\
\hline pBQ200 & none & NG $^{\mathrm{c}}$ & $211 \pm 11$ & $509 \pm 44$ & $321 \pm 80$ \\
pGP529 & wild type & $7 \pm 4$ & $26 \pm 8$ & $562 \pm 85$ & $423 \pm 100$ \\
pGP852 & RocG-SR1, I308T & NG & $9 \pm 2$ & $207 \pm 24$ & $15 \pm 5$ \\
pGP853 & RocG-SR2, S234R & NG & $5 \pm 2$ & $164 \pm 31$ & $9 \pm 6$ \\
pGP854 & RocG-SR3, E93K & NG & $9 \pm 2$ & $140 \pm 42$ & $18 \pm 11$ \\
pGP933 & RocG-SR5, Y158H & NG & $8 \pm 4$ & $187 \pm 25$ & $17 \pm 2$ \\
pGP932 & RocG-MF, D122N & $277 \pm 23$ & $505 \pm 58$ & $650 \pm 62$ & $840 \pm 134$ \\
\hline
\end{tabular}

a $\mathrm{SR}$ and MF denote the superrepressor and monofunctional RocG variants, respectively.

b gltA expression is expressed in units per milligram of protein. All measurements were performed at least three times. Average values with standard deviations are shown.

${ }^{\text {c }} \mathrm{NG}$, no growth. 


\section{Enzymatic activities of mutant glutamate dehydrogenases}

When we determined the activity of GltC in the strains expressing the mutant alleles of $\operatorname{roc} G$, we observed that strains expressing the superrepressor variants were unable to grow with arginine as the single carbon source. This might result from a reduced or absent enzymatic activity of these RocG variants; consequently, we studied the enzymatic activity of RocG and its mutant variants in more detail.

First, we analyzed the growth of B. subtilis GP28 carrying the different rocG alleles on CR minimal medium in the presence or in the absence of glucose. Strains bearing the empty vector pBQ200 or pGP529 (wild-type RocG) were used as controls. Both strains grew well on a medium containing both glucose and arginine. Similarly, all strains expressing the mutant RocG proteins grew well on this medium, suggesting that these strains also have full biosynthetic capacity required for growth on minimal medium (see Tab. 2.3). The strain expressing wild-type RocG was also able to grow with arginine as the only source of carbon and energy. By contrast, the strain carrying the empty vector did not grow on this medium because it lacks a functional glutamate dehydrogenase required for the last step of arginine catabolism, the conversion of glutamate into 2-oxoglutarate (Commichau et al., 2008). The strains expressing the superrepressor RocG variants were unable to utilize arginine as the only carbon source, suggesting that these RocG variants are severely impaired in their enzymatic activity. The monofunctional RocG protein that was originally isolated on CR plates allowed arginine utilization, suggesting that this protein is enzymatically active.

Next, we purified recombinant RocG variants, and their enzymatic activities were determined. Wild-type RocG exhibited catabolic and anabolic activities of 3.9 and 39.7 $\mathrm{U} / \mathrm{mg}$ protein, respectively. The catabolic activities of all superrepressor proteins were severely reduced (see Tab. 2.3), in agreement with the inability of the corresponding B. subtilis strains to grow with arginine as the only carbon source. In addition to their reduced activities, these enzymes have a drastically increased $K_{m}$ for glutamate (between 23 and $75 \mathrm{mM}$, compared to $2.9 \mathrm{mM}$ for the wild-type protein), indicating a reduced affinity for the substrate. Similarly, with the exception of the RocG(E93K) variant, the anabolic activities and the $\mathrm{K}_{\mathrm{m}}$ values for ammonium of the superrepressors were reduced and increased, respectively, albeit to a lesser extent (see Tab. 2.3). The monofunctional RocG variant had a 4-fold higher $\mathrm{K}_{\mathrm{m}}$ for glutamate than for the wildtype enzyme; however, this variant still enabled the parent strain GP28 to grow with 
arginine as the single carbon source. Similarly, the anabolic properties of this RocG mutant are not significantly altered in comparison to the wild-type enzyme, and confirm that the D122N substitution in RocG predominantly affects the inhibitory interaction of RocG with GltC, but not its enzymatic properties. 
Tab. 2.3 - Enzymatic activities of the RocG variants.

\begin{tabular}{|c|c|c|c|c|c|c|c|}
\hline \multirow[t]{2}{*}{ Plasmid $^{\text {a }}$} & \multirow[t]{2}{*}{ RocG variant } & \multicolumn{2}{|c|}{ Growth on plates } & \multirow{2}{*}{ 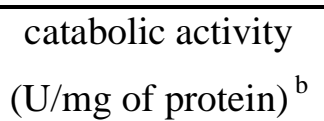 } & \multirow{2}{*}{$\begin{array}{c}\mathrm{K}_{\mathrm{M}} \text { (glutamate) } \\
(\mathrm{mM})^{\mathrm{b}}\end{array}$} & \multirow{2}{*}{ 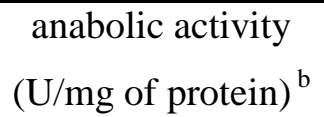 } & \multirow{2}{*}{$\begin{array}{c}\mathrm{K}_{\mathrm{M}} \text { (ammonium) } \\
(\mathrm{mM})^{\mathrm{b}}\end{array}$} \\
\hline & & CR-Glc & $\mathrm{CR}$ & & & & \\
\hline pBQ200 & none & +++ & - & $\mathrm{NA}^{\mathrm{c}}$ & NA & NA & NA \\
\hline pGP529 & wild type & +++ & + & $3.9 \pm 0.67$ & $2.9 \pm 1.25$ & $39.7 \pm 2.52$ & $18 \pm 5$ \\
\hline pGP852 & RocG-SR1, I308T & +++ & - & $\mathrm{ND}^{\mathrm{d}}$ & ND & ND & ND \\
\hline pGP853 & RocG-SR2, S234R & +++ & - & $0.12 \pm 0.03$ & $74.7 \pm 7$ & $4.3 \pm 1.28$ & $58.7 \pm 1.53$ \\
\hline pGP854 & RocG-SR3, E93K & +++ & - & $0.4 \pm 0.06$ & $46.3 \pm 4.16$ & $36 \pm 7.54$ & $40 \pm 3$ \\
\hline pGP933 & RocG-SR5, Y158H & +++ & - & $0.69 \pm 0.17$ & $23 \pm 3.61$ & $14.3 \pm 3.51$ & $38.7 \pm 3.60$ \\
\hline pGP932 & RocG-MF, D122N & +++ & + & $2.27 \pm 0.32$ & $12 \pm 2.65$ & $37 \pm 3.61$ & $10.3 \pm 2.52$ \\
\hline
\end{tabular}

${ }^{a}$ These plasmids were used for the growth experiments.

${ }^{\mathbf{b}}$ The experiments were performed in triplicate. Average values with standard deviations are shown.

${ }^{c}$ NA, not applicable.

${ }^{\mathbf{d}} \mathrm{ND}$, not determined due to low stability of this protein.

Note that the assays for catabolic and anabolic activities were performed with an excess of ammonium and glutamate, respectively. In vivo, RocG is unable to perform the anabolic reaction most likely due to its low affinity for ammonium. 


\section{Crystal structures of RocG(E93K) and GudB1}

In an attempt to gain a better molecular understanding of structure-function relationships among the different mutant RocG alleles, we have determined the crystal structures of the superrepressor RocG(E93K) mutant and the decryptified secondary glutamate dehydrogenase GudB1. This protein lacks the three-amino-acid insertion in the active site that renders the GudB protein completely inactive (Belitsky \& Sonenshein, 1998; Commichau et al., 2007b). During the crystallographic aspect of this study, a number of RocG variants were purified and subjected to crystallization trials. The wild-type enzyme and most of the mutants studied produced comparatively small crystals that diffracted to $4 \AA$ at best, whereas the RocG(E93K) crystals diffracted to a resolution of $2.3 \AA$ and belonged to space group $\mathrm{P} 2{ }_{1} 2_{1} 2_{1}$. GudB 1 crystals diffracted to $2.4 \AA$ and belonged to space group $\mathrm{P} 2{ }_{1}$. Both structures were solved by molecular replacement with six subunits in the asymmetric unit and were refined until convergence. The final RocG(E93K) model contains 2409 out of a possible 2544 residues with 5 polyethylene glycol (PEG) ions and 829 water molecules, whereas the GudB1 model contains 2354 out of a possible 2538 residues and 414 water molecules. Both structures have been restrained to standard bond lengths and angles, with over $99 \%$ of residues lying in the most favored and additionally allowed regions of the Ramachandran plot, (Ramachandran et al., 1963) as calculated by the program PROCHECK (Laskowski et al., 1993). A full summary of the data collection, processing, and refinement statistics can be found in Tab. 2.4.

\section{$\operatorname{RocG(E93K)}$}

Overall, the electron density for RocG(E93K) is of high quality throughout all six molecules, with the exception of the $\mathrm{N}$-terminal 15 residues that were not visible in the electron density, have been omitted from the model in chains B, C, D, E, and F, and are only partially included in chain A. Similarly, the entire length of chains A and B could be traced in the electron density with high confidence, whereas in the regions corresponding to residues $215-342$ in chains $\mathrm{C}-\mathrm{F}$, the electron density is of poorer overall quality, and residues $274-276$ in chain D, residues $270-286$ in chain E, and residues $270-285$ in chain $\mathrm{F}$ have been omitted from the final model. 
Tab. 2.4 - Data collection and refinement statistics.

\section{RocG(E93K)}

\section{Data collection}

Space group

Unit cell parameters

$$
\begin{aligned}
& a, b, c,(\AA) \\
& \alpha, \beta, \gamma,\left(^{\circ}\right)
\end{aligned}
$$

Wavelength $(\AA)$

Resolution $(\AA)$

$\mathrm{R}_{\text {merge }}(\%)^{\mathbf{a}}$

$I / \sigma I$

Completeness (\%)

Redundancy

\section{Refinement}

Resolution (Å)

No. reflections

$\mathrm{R}_{\text {work }} / \mathrm{R}_{\text {free }}(\%)$

No. Atoms

Protein

Water

Ligand/Ion

B factors

Protein

Water

Ligand/Ion

R.m.s. deviations

90, 90, 90

0.98

$6.3(44.5)$

$16.0(3.1)$

$99.8(100.0)$

$4.2(4.3)$

$20.0-2.3$

142264

$19.1 / 24.3$

19609

824

35

56.0

48.1

56.0

\section{GudB1}

P 21

86.1, 158.0, 193.8

90, 118.7, 90

0.98

$20-2.3(2.42-2.30)$

$50.0-2.4(2.46-2.40)$

$7.0(38.0)$

$11.6(3.2)$

$97.8(97.8)$

$3.6(3.7)$

$50.0-2.4$

98133

$24.7 / 27.8$

15594

414

0

45.5

40.2 


\begin{tabular}{|c|c|c|}
\hline & RocG(E93K) & GudB1 \\
\hline Bond lengths $(\AA)$ & 0.014 & 0.003 \\
\hline Bond angles $\left({ }^{\circ}\right)$ & 1.44 & 0.82 \\
\hline PDBid & $3 \mathrm{~K} 92$ & $3 \mathrm{~K} 8 \mathrm{Z}$ \\
\hline
\end{tabular}

Values in parentheses are for the highest-resolution shell.

${ }^{\mathrm{a}} R_{\mathrm{merge}}=\Sigma_{h k l} \Sigma_{i}\left|I_{i}-I_{\mathrm{m}}\right| / \Sigma_{h k l} \Sigma_{i} I_{i}$, where $I_{i}$ and $I_{\mathrm{m}}$ are the observed intensity and mean intensity of related reflections, respectively.

The structure of RocG(E93K) is very similar to those of a number of glutamate dehydrogenases studied to date, being hexameric with a subunit comprising two domains separated by a cleft, which forms the active site (Yip et al., 1995; Baker et al., 1997; Knapp et al., 1997; Britton et al., 1999; Nakasako et al., 2001). Domain I comprises residues 1-193 at the N-terminus and the final 17 residues at the C-terminus, and forms a typical amino acid dehydrogenase fold with a central six-stranded mixed $\beta$ sheet (strand order $\mathrm{a} \uparrow, \mathrm{b} \downarrow, \mathrm{d} \uparrow, \mathrm{c} \uparrow, \mathrm{e} \uparrow, \mathrm{f} \uparrow$ ) flanked by two $\alpha$-helices on one side and by one $\alpha$ - helix on the other side. Domain II is composed of a central seven-stranded parallel $\beta$-sheet (strand order $\mathrm{m}, 1, \mathrm{k}, \mathrm{g}, \mathrm{h}, \mathrm{i}, \mathrm{j}$ ) flanked by four $\alpha$-helices on one side and by three $\alpha$-helices on the other side, and forms a variant of the common NAD(P)binding Rossmann fold (Rossmann et al., 1974). The two domains are connected by a number of long $\alpha$-helices that converge upon a single hinge region, allowing for relatively large domain motions to occur (up to $14 \AA$ ). Indeed, these domain motions are critical to the catalytic mechanism of this enzyme (Stillman et al., 1999) and are believed to allow the nicotinamide ring of the nucleotide cofactor to come into contact with the $\mathrm{C}^{\alpha}$ of the glutamate substrate in order for hydride transfer to occur.

The six subunits in the asymmetric unit form a single compact homohexamer with 32-point group symmetry, and the vast majority of intersubunit interactions around the 2-fold and 3-fold axes are provided by domain I. The six chains in the asymmetric unit represent, to varying degrees, the open and closed forms of glutamate dehydrogenase. Chain A represents a more closed conformation, and chains B, C, D, E, and F are more open, with maximal displacements of $14 \AA$ in comparison to chain A. These differences in domain architecture can be explained in part by crystal contacts within the lattice, 
with domain II of chain A making extensive contacts with a symmetry-related copy of chain C. Furthermore, the absence of crystal contacts in chains C, D, E, and F may account for the disordered residues at the periphery of domain II. While the closed form of the enzyme is usually associated with glutamate binding (Stillman et al., 1999), this kind of intersubunit variation has already been observed in the apo form of the homologous enzyme from Thermococcus profundus (Nakasako et al., 2001

Neither glutamate nor any other substrate was added to the RocG crystals, and examination of the $2 \mathrm{~F}_{\mathrm{obs}}-\mathrm{F}_{\text {calc }}$ and $\mathrm{F}_{\mathrm{obs}}-\mathrm{F}_{\text {calc }}$ electron density maps in the active site did not reveal any significant unexplained density.

The superrepressor E93K variant of RocG described above is, we believe, virtually identical in structure to the wild-type enzyme. The mutation, which changes a glutamate into a lysine, is located within the first turn of the third helix of domain I, within an acidic stretch of amino acids. The local structure reveals no significant differences when compared to other bacterial glutamate dehydrogenases or to GudB1. Indeed, the high overall level of structural similarity between RocG(E93K) and GudB1 (r.m.s.d., $0.95 \AA$ ) indicates that the RocGE93K structure is an appropriate template with which to investigate the other mutants described above. Wild-type RocG could be crystallized under the same conditions as $\operatorname{RocG}(\mathrm{E} 93 \mathrm{~K})$, and although the diffraction obtained from these morphologically identical crystals was limited to $4 \AA$, their growth indicates that the structural differences arising from the mutation are minor. 


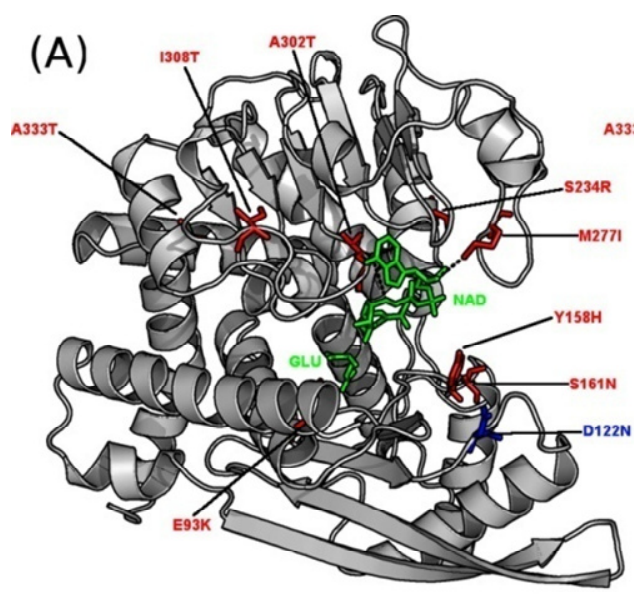

(B)
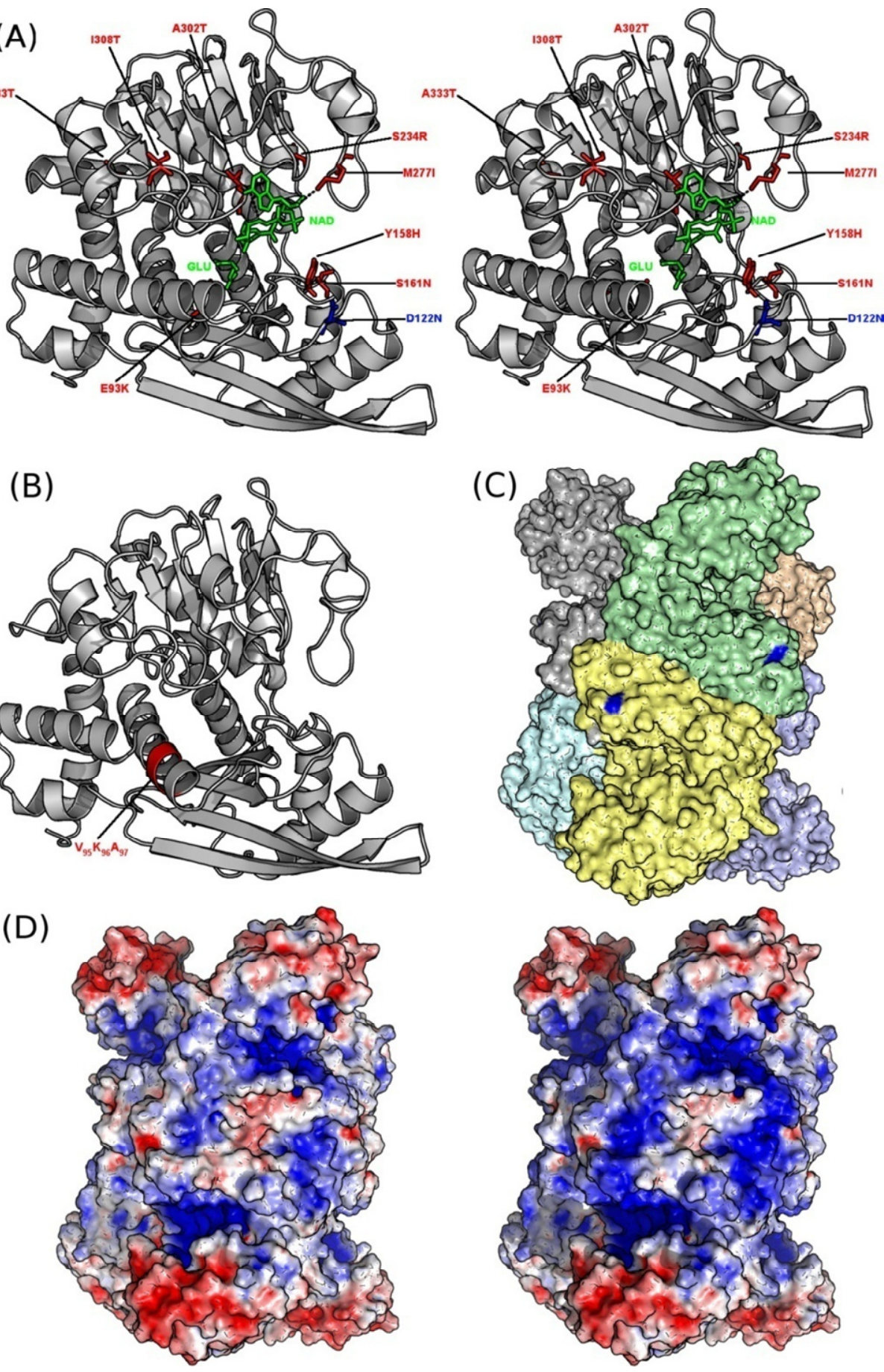

Fig. 2.4 - Structural organization of RocG/GudB1 variants.

(A) Stereo view of the RocG monomer shown in cartoon format, with superrepressor mutations highlighted in red and with the single monofunctional variant highlighted in blue. For reference, the nucleotide cofactor and the glutamate substrate are shown in green in their expected positions in the active site based on structural superpositions with the C. symbiosum glutamate dehydrogenase-NAD 
complex (Stillman et al., 1993) and the P. islandicum glutamate dehydrogenase-glutamate complex, (Bhuiya et al., 2005) respectively. Direct interactions between the superrepressor mutants and the substrate are shown as dotted lines. (B) The GudB1 monomer, viewed in the same orientation as RocG in (A). The three residues that are directly repeated in the B. subtilis 168 strain but are present only once in the "wild type" are highlighted in red. (C) The surface of the RocG hexamer viewed along the 2-fold axis, with each chain colored individually. The monofunctional D122N mutation is shown in blue and locates to a possible interaction surface centered on the molecular 2-fold axis. (D) Analysis of the effect of the D122N substitution on the surface electrostatics of RocG. The left panel shows wild-type RocG, while the right panel shows the D122N-substituted variant; both variants are shown in the same orientation as in Fig. 4c, with electrostatic calculations performed by the program APBS (Baker et al. 2001) and contoured at $\pm 5 K_{\mathrm{b}} T / e_{\mathrm{c}}$.

The majority of the mutated residues of the other RocG variants isolated in this study, are surface exposed and cluster to the immediate surroundings of the active-site cleft. Superposition of the structures of glutamate dehydrogenase from Clostridium symbiosum (Stillman et al., 1993) and Pyrobaculum islandicum (Bhuiya et al., 2005) has allowed us to model the expected position of the substrate and the nucleotide cofactor, respectively. Six out of 10 of these mutants (Glu93, Tyr158, Ser234, Met277, Ala302, and Ala303) are within $8 \AA$ of the expected position of the nucleotide cofactor (with respect to the closed form of the enzyme), and three of these (Ala302, Ala303, and Met277) are in positions where they are able to make direct van der Waals interactions (Fig. 2.4A) On the other hand, the D122 residue, at which a mutation to asparagine increases the $\mathrm{K}_{\mathrm{m}}$ for glutamate, is not within the active-site groove; instead, it is over $12 \AA$ away from either nucleotide or substrate and solvent exposed, facing towards a shallow depression that surrounds the molecular 2-fold axis (Fig. 2.4C).

\section{GudB1}

Similarly, the GudB1 variant used in this study is a mutant with respect to the laboratory strain B. subtilis 168 , but this allele is found in environmental isolates and can thus be regarded as "wild type" (Zeigler et al., 2008). The laboratory strain contains an insertion of the three-residue repeat $\mathrm{V}_{95} \mathrm{~K}_{96} \mathrm{~A}_{97}$, which is located within the third helix of domain I (Fig. 4b). This insertion presumably causes severe destabilization of the fold of the protein, leading to an inactive enzyme that is very quickly degraded; consistent with this premise, recombinant GudB from B. subtilis 168 cannot be purified 
in our hands. This is supported by the extremely rapid proteolytic degradation of GudB in B. subtilis (Gerth et al., 2008). By contrast, GudB1 is an active glutamate dehydrogenase with enzymatic activities of 6.7 and $24.5 \mathrm{U} / \mathrm{mg}$ of protein for catabolic and anabolic reactions, respectively. The $\mathrm{K}_{\mathrm{m}}$ values were found to be $17.9 \mathrm{mM}$ for glutamate and $41 \mathrm{mM}$ for ammonium.

Both the open form and the closed form of GudB1 were evident in the six molecules in the asymmetric unit (two closed subunits and four open subunits), which associate to form the same compact homohexamer as RocG. This variation indicates that the closed conformation is quite readily adopted under different crystal forms. As would be expected given the $74 \%$ sequence identity between RocG and GudB1, their structures are very similar: the 'open' conformers can be superimposed on 409 aligned residues to yield an r.m.s.d. of $0.95 \AA$ A. A comparison of the 'closed' conformers of RocGE93K and GudB1 yields an r.m.s.d. of $1.2 \AA$, whereas that between the 'open' form and the 'closed' form yields an r.m.s.d. of $2.5 \AA$.

\section{Discussion}

In this study, we have isolated and characterized a set of RocG variants that uncouple the enzymatic and regulatory activities of this trigger enzyme. Several mutations result in loss of enzymatic activity and are accompanied by permanent substrate-independent inactivation of GltC. A second class, exemplified by one mutant protein, has retained enzymatic activity but has lost the ability to inactivate GltC. It has been shown that the catalytically active glutamate dehydrogenases RocG and GudB1 are able to inactivate GltC and thereby prevent transcription activation of glutamate biosynthetic genes (Belitsky \& Sonenshein, 2004; Commichau et al., 2007a; Commichau et al., 2007b). Moreover, the cofactor glutamate is required to elicit the formation of the inhibitory forms of RocG or GudB1 (Commichau et al., 2007a). Similarly, the B. subtilis glutamine synthetase binds the transcription factors TnrA and GlnR if the enzyme is feedback inhibited by glutamine (Wray et al., 2001; Wray \& Fisher, 2005).

The separation of enzymatic and regulatory activities has already been observed for other trigger enzymes such as the B. subtilis glutamine synthetase. As shown in this 
study for RocG, many of these mutations cluster to the region of the active site of the glutamine synthetase (Wray \& Fisher, 2005; Fisher \& Wray, 2009). We showed that the mutations causing the superrepressor phenotype reduce the catalytic activity of RocG (see Tab. 2.3). Thus, one could assume that the conformation of these RocG variants required for GltC inhibition is more stable than that of the wild-type RocG enzyme. These variants might require less glutamate to elicit the "inhibitory" conformation of glutamate dehydrogenase. By contrast, the monofunctional RocG enzyme is enzymatically active, but glutamate does not induce the inhibitory conformation required to inactivate GltC activity.

Dual effects on regulatory interactions have also been observed for sugar permeases of the B. subtilis phosphotransferase system, which phosphorylate and thereby inactivate transcriptional anti-terminators and activators that control the expression of the permeases. Mutations that result in complete inactivation of the permeases (such as deletion of corresponding genes) result in the constitutive activity of the corresponding transcription factors. Similarly, mutations affecting the phosphorylation of the anti-terminators or activators by the sugar permeases (such as mutations of the phosphorylation sites) lead to permanent activity of the transcription factors and concomitant loss of sugar transport activity. By contrast, mutations that affect the membrane-bound transport domain lock the permeases in the phosphorylated state, since the phosphoryl group can never be transferred to the incoming sugar. These permease variants phosphorylate and thereby inactivate their cognate regulator proteins even in the presence of the normally inducing sugar in the medium. Such mutations have been intensively studied for the B. subtilis fructose permease (LevDEFG) and the glucose permease PtsG, which control the activities of the transcription activator LevR and the transcriptional anti-terminator GlcT, respectively (Stülke et al., 1997; Bachem \& Stülke, 1998; Martin-Verstraete et al., 1998; Schmalisch et al., 2003). In E. coli, proline dehydrogenase acts as the repressor for its own transcription in the absence of the substrate proline. In this case, the link between the two activities of the protein is more intricate, and mutations that lead to permanent repression of putA expression result from loss of proline binding and, thus, enzymatic activity (Muro-Pastor \& Maloy, 1995).

Mutations affecting the control of gene expression by trigger enzymes have not only been found in the genes encoding the trigger enzymes. Similarly, mutations 
affecting the controlled transcription factors may interfere with their productive interaction with the cognate trigger enzyme. Such mutations have been isolated for GltC, and reduced interaction with RocG has been demonstrated for one of these variants (Belitsky \& Sonenshein, 1995; Commichau et al., 2007b).

An interesting result of this study is the identification of 10 different superrepressor variants of RocG, while only one monofunctional protein incapable of inhibiting GltC was found. Originally, we would have expected to find more monofunctional proteins than superrepressors. The small number of monofunctional RocG variants isolated might indicate that the GltC interaction surface is relatively large and involves multiple side chains, and that the interaction between the two proteins and the inhibition of GltC by RocG cannot be easily disrupted by single amino acid substitutions in RocG. The location of Asp122 on the surface of the enzyme, close to a shallow depression that surrounds the 2-fold axis of RocG (Fig. 2.4C), suggests that this region may be involved in the interaction of RocG with GltC. Indeed, it could be envisaged that this 2-fold axis is coincident with one of the 2-fold axes of GltC, which, based on structural studies of other LysR-type transcriptional regulators, is believed to be a tetramer with two 2-fold axes (Maddocks \& Oyston, 2008). Furthermore, the fact that the substitution of this residue with an isosteric asparagine is capable of disrupting the inactivation of GltC by $\operatorname{RocG}(\mathrm{E} 93 \mathrm{~K})$ indicates that the charge properties of this residue are more important than its shape and size. This may be explained by the fact that in the RocG(E93K) structure, Asp122 forms an ion pair with Arg124; this interaction would most likely be disrupted in the D122N mutation, perhaps causing a larger alteration in the surface properties of the enzyme than would otherwise be expected. Analysis of the impact of this substitution on the surface electrostatics of RocG using the program APBS (Baker et al., 2001) reveals a significant increase in the basic character of the region surrounding the shallow depression, perhaps indicating how an isosteric substitution could cause a large disturbance in an interaction surface (Fig. 2.4D). On the other hand, any mutation that interferes with enzymatic activity without drastic changes in the overall structure of the protein might lead to permanent inhibition of GltC. This idea is supported by the clustering of the superrepressor mutations around the position of the nucleotide cofactor (Fig. 2.4A) and is also in good agreement with the large number of such mutations. 
In most bacteria, glutamate dehydrogenase is required for ammonium assimilation. In E. coli, the enzyme can synthesize glutamate at high ammonium concentrations due to the low affinity for this substrate (Reitzer, 2003). In B. subtilis, the glutamate synthase encoded by the gltAB operon is the only enzyme for ammonium assimilation. Even the decryptified GudB1 protein is unable to catalyze glutamate formation in vivo (Commichau et al., 2008). The reason for this difference between the glutamate dehydrogenase of E. coli and the glutamate dehydrogenase of B. subtilis remains opaque. The data presented herein do, however, provide an explanation for the exclusive catabolic activity of the B. subtilis enzyme: glutamate dehydrogenase of E. coli, with a $\mathrm{K}_{\mathrm{m}}$ of $2.5 \mathrm{mM}$ for ammonium (Sharkey \& Engel, 2008), is only active at high ammonium concentrations (Reitzer, 2003). However, the $\mathrm{K}_{\mathrm{m}}$ values of the B. subtilis enzymes are even higher (18 and $41 \mathrm{mM}$ for RocG and GudB1, respectively). The ammonium concentration in the cell is restricted by an active transport system. At high extracellular ammonium concentrations, the very small fraction that is present as ammonia diffuses freely into the cell, whereas at low ammonium concentrations, the ammonium transporter $\mathrm{NrgA}$ is expressed, and ammonium is taken up from external sources (Wray et al., 1994; Detsch \& Stülke, 2003).

Thus, even high external ammonium concentrations do not result in high internal concentrations that would be sufficient for significant glutamate biosynthesis by the glutamate dehydrogenase.

The results presented in this study support the idea of an inhibitory interaction between the glutamate dehydrogenase RocG and the transcription factor GltC. Moreover, they provide new insights into the relation between the enzymatic activities and the regulatory activities of the trigger enzyme RocG. The identification of the monofunctional RocG variant that has lost its regulatory function but has retained enzymatic activity is in excellent agreement with the previous conclusion that the enzymatic activity of RocG is important but not sufficient for the control that it exerts on GltC. The availability of mutations in both partners that interfere with the inhibition of GltC by RocG will be of great value in the elucidation of the molecular details behind this regulatory interaction. 


\section{Acknowledgements}

We are grateful to Henrike Pförtner for her help with the isolation of mutants. Colin Harwood is acknowledged for helpful discussions. The authors acknowledge the staff of the beamlines at the Diamond synchrotron light source for their help during diffraction data collection. This work was supported by grants from the Deutsche Forschungsgemeinschaft and the Federal Ministry of Education and Research SYSMO network (PtJ-BIO/0313978D and 0313978A) to J.S. and by SYSMO grants from the UK Biotechnology and Biological Sciences Research Council to R.J.L. F.M.C. and J.S. were additionally supported by the Fonds der Chemischen Industrie. 


\section{Chapter 3}

Analysis of the RocG-GltC interaction 


\begin{abstract}
In the glutamate metabolism of $B$. subtilis the glutamate dehydrogenase RocG fulfills two functions. First, RocG catalyzes the deamination of glutamate to 2oxoglutarate that serves as a source of carbon. Second, RocG acts as a trigger enzyme by controlling the activity of the transcriptional regulator GltC. The activity of GltC is essential for the expression of the gltAB operon, encoding the glutamate synthase (GOGAT). In the presence of glutamate or its precursor arginine RocG prevents GltC from the induction of GOGAT expression, thus a futile cycle of glutamate synthesis and degradation is avoided. Two classes RocG variants were characterized. The first class is drastically diminished in enzymatic activity but strongly inhibits GltC. The second class consists of a mutant RocG protein that lost the capacity to control GltC activity but remained enzymatically active.

This study provides information about the interaction between the glutamate dehydrogenase RocG and the transcriptional activator GltC. It is suggested that the mode of interaction involves more factors. Moreover, the capacity of the mutant RocG variants to physically interact with GltC was tested in B. subtilis. The monofunctional RocG protein that was shown to fail in inhibiting the GltC protein still interacts with GltC. This result leads to the assumption that interaction is not the equivalent of inhibition and the mechanism of RocG to trigger the GltC activity might be more complex.
\end{abstract}

\title{
Introduction
}

In $B$. subtilis glutamate metabolism is one of the most tightly regulated pathways. Briefly, glutamate is exclusively synthesized by the combined reactions of the glutamine synthetase and the glutamate synthase (GltAB) in the GOGAT cycle (see Fig. 1.1A) (Fisher, 1999). The catabolic reaction, the oxidative deamination of glutamate to 2-oxoglutarate and ammonium is performed by the glutamate dehydrogenase (GDH), RocG (Belitsky \& Sonenshein, 1998). This enzyme is only capable of glutamate degradation (Belitsky \& Sonenshein, 1998, Commichau et al., 2008). The expression of the gltAB operon and the $\operatorname{roc} G$ gene is mutually exclusive (Commichau et al., 2007b). In the absence of arginine and in the presence of glucose, the $\operatorname{roc} G$ gene is subject to 
catabolite repression. Under this condition the gltAB operon is expressed due to the activation by the transcriptional regulator GltC. In the absence of glucose and the presence of arginine, the $\operatorname{roc} G$ gene is strongly transcribed. Then, the RocG protein sequesters the transcriptional regulator GltC in the presence of its substrate glutamate which prevents the expression of the gltAB operon (Commichau et al., 2007a) (see Fig. 2.1B). Besides the positive effect of GltC on the gltAB expression, GltC exerts autorepression on the $g l t C$ gene that is divergently transcribed with respect to the gltAB operon (Belitsky et al., 1995; Belitsky \& Sonenshein, 1995). This autoregulation has not been shown to be dependent on the nitrogen source (Belitsky \& Sonenshein, 1995).

In this work mutant variants of the trigger enzyme RocG were isolated, that show a different inhibition pattern concerning the GltC protein (Chapter 2). Two classes of variants were isolated. Firstly, the so called superrepressor RocG proteins (RocG-SR) were characterized. These RocG-SR proteins strongly inhibit GltC if glutamate or its precursor arginine is available but show lower enzymatic activity than the wild type protein. Secondly, a monofunctional RocG protein (RocG-MF) was isolated. This protein is fully active as a glutamate dehydrogenase but lost the capacity to inhibit GltC. All mutant proteins are based on a single amino acid exchange that alters the trigger enzyme RocG in a protein with only one function. In the RocG-SR proteins the enzymatic activity is abolished but they still regulate GltC activity, whereas the RocGMF protein completely lost the ability to control GltC but this protein is still active as a glutamate dehydrogenase.

In this study, mainly two questions are addressed. On the one hand it was analyzed whether the RocG-GltC interaction can be detected in the bacterial two-hybrid system. This approach might give information about the mode of interaction. The result can provide hints whether the interaction is binary or whether other factors are involved. On the other hand the ability of the mutant RocG proteins to interact with GltC in vivo was investigated in a SPINE approach. 


\section{Materials and Methods}

\section{Bacterial strains and growth conditions}

E. coli DH5 $\alpha$ and BTH101 (Sambrook et al., 1989; Karimova et al., 1998) were used for cloning experiments and bacterial two-hybrid (B2H) analyses, respectively. The E. coli strains were grown in LB medium. The B. subtilis strains GP717 (trpC2 $\Delta$ gudB::cat rocG::Tn10 spc amyE::(gltA-lacZ aphA3) gltB1 ansR-C107A) (Commichau et al., 2008) and GP28 (trpC2 rocG::Tn10 spc $\Delta$ gudB::cat amyE::gltA'-'lacZ aphA3) (Commichau et al., 2007b) were grown in SP medium, LB medium or in C minimal medium supplemented with tryptophan (at $50 \mathrm{mg} / \mathrm{l}$ ) (Wacker et al., 2003). CSE medium is $\mathrm{C}$ minimal medium supplemented with sodium succinate $(6 \mathrm{~g} / \mathrm{l})$ and potassium glutamate $(8 \mathrm{~g} / \mathrm{l})$. C-Glc is $\mathrm{C}$ minimal medium supplemented with glucose $(5 \mathrm{~g} / \mathrm{l})$, and CS is supplemented with sodium succinate (6 g/l) (Wacker et al., 2003). CR medium is supplemented with $5 \mathrm{~g} / \mathrm{l}$ arginine. LB and SP and were prepared by the addition of $17 \mathrm{~g}$ Bacto agar/1 (Difco, Lawrence, USA) to LB or SP medium, respectively.

\section{In vivo detection of protein-protein interactions}

The isolation of protein complexes from B. subtilis cells was performed by the SPINE technology (Herzberg et al., 2007). To express the RocG protein and its derivatives fused to an N-terminal Strep-tag, the different $\operatorname{roc} G$ alleles were amplified (for primers see Tab. S1) and the resulting PCR products were cloned into the expression vector pGP380. The PCR products were digested with BamHI and HindIII and ligated to vector pGP380 (Herzberg et al., 2007). The resulting plasmids are pGP1708 (RocG), pGP1709 (RocG-MFD122N). These plasmids and pGP652 (RocGSR3 E93K) (Tholen, 2008) were used to transform B. subtilis GP717 (Commichau et $a l ., 2008)$. For cultivation one liter culture was inoculated to an $\mathrm{OD}_{600}$ of 0.1 with an overnight culture. This culture was grown at $37^{\circ} \mathrm{C}$ until $\mathrm{OD}_{600} 0.9-1.0$ and divided. One half and aliquots of $1.5 \mathrm{ml}$ for the $l a c Z$ assay were harvested immediately, and the other was treated with formaldehyde (6 g/l, $20 \mathrm{~min})$ to facilitate the cross-linking (Herzberg et al., 2007). After cross-linking, the cells were also harvested and washed with a buffer containing $50 \mathrm{mM}$ Tris- $\mathrm{HCl}(\mathrm{pH} 7.5)$ and $200 \mathrm{mM} \mathrm{NaCl}$. The pellets were lysed using a French press (20,000 p.s.i., 138,000 kPa; Spectronic Instruments, Garforth UK). After 
lysis the crude extracts were centrifuged at $100,000 \mathrm{~g}$ for $1 \mathrm{~h}$. For purification of the Strep-tagged proteins the resulting supernatants were passed over a Streptactin column (IBA, Göttingen, Germany; $0.5 \mathrm{ml}$ bed volume). The recombinant proteins were eluted with desthiobiotin (IBA, Göttingen, Germany, final concentration $2.5 \mathrm{mM}$ ). Aliquots of the different fractions were subjected to SDS-PAGE. Prior to electrophoresis, the protein samples were boiled for $20 \mathrm{~min}$ in Laemmli buffer to reverse the cross-links. As a control, the B. subtilis strain GP717 carrying the empty vector pGP380 was used.

\section{B2H assay}

The bacterial two-hybrid (B2H) assay was used to analyze the protein-protein interactions between RocG and GltC (Karimova et al., 1998). The B2H system is based on the interaction-mediated reconstruction of adenylate cyclase $(\mathrm{CyaA})$ activity from Bordetella pertussis in E. coli. The CyaA enzyme consists of two complementary fragments T18 and T25 that are not active when physically separated. Fusion of these fragments to interacting proteins results in functional complementation between the T18 and T25 fragments and the synthesis of cAMP. cAMP production can be monitored by measuring the $\beta$-galactosidase activity of the cAMP-CAP-dependent promoter of the E. coli lac operon. Thus, a high $\beta$-galactosidase activity reflects the interaction between the hybrid proteins. Plasmids pUT18 and p25-N allow the expression of proteins fused to the N-terminus of the T18 and T25 fragments of the CyaA protein, respectively, and the plasmids pUT18C and pKT25 allow the expression of proteins fused to the Cterminus of the T18 and T25 fragments of the CyaA protein, respectively (Karimova et al., 1998; Claessen et al., 2008). The plasmids pKT25-zip and pUT18C-zip served as positive controls for complementation. These plasmids express T18-zip and T25-zip fusion proteins that can associate due to the leucine zipper motifs resulting in an active CyaA enzyme and a high $\beta$-galactosidase activity. DNA fragments of the $\operatorname{roc} G$ and gltC genes were obtained by PCR and digested with the enzymes KpnI and XbaI (for primers, see Tab. S1). The PCR products were cloned into the four vectors of the twohybrid system digested with the same enzymes, respectively. The resulting plasmids (see Tab. S2) were used for cotransformations of E. coli BTH101 and the proteinprotein interactions were then analyzed by plating the cells on LB plates containing ampicillin $(100 \mathrm{mg} / \mathrm{ml})$, kanamycin $(50 \mathrm{mg} / \mathrm{ml}), \mathrm{X}-\mathrm{Gal}(40 \mathrm{mg} / \mathrm{ml})$ (5-bromo-4-chloro- 
3-indolyl- $\beta$-D-galactopyranoside) and IPTG (0.5 mM) (isopropyl- $\beta$-D-thiogalactopyranoside), respectively. The plates were incubated for a maximum of $48 \mathrm{~h}$ at $30^{\circ} \mathrm{C}$.

\section{Materials and Methods Summary}

\section{Western blot analysis}

Western blot analysis of GltC was performed as described previously (Gunka et al., 2010). GltC were detected with rabbit polyclonal antiserum raised against B. subtilis GltC (Commichau et al., 2007a).

\section{Activity of the gltA-lacZ fusion}

Quantitative assays of lacZ expression in B. subtilis were performed as described in Gunka et al., subm.

\section{DNA manipulation}

All primer sequences are provided in Tab. S1. Methods used for DNA manipulation were performed as described previously (Gunka et al., 2010). 


\section{Results}

\section{RocG-GItC interaction in the bacterial two-hybrid assay: Limits of this method}

A variety of experiments were performed to characterize the Roc-GltC interaction in more detail. But none of the methods used (e.g. surface plasmon resonance (SPR), electrophoretic mobility shift assay (EMSA) data not shown) was successful in giving new insights into the mechanism of interaction (J. A. Newman, R. J. Lewis, F. M. Commichau, C. Herzberg, pers. comm.).

To test whether the protein-protein interaction between RocG and GltC can be reconstructed heterologously in E. coli, both protein were fused to the C- and Nterminus of the T18 and T25 domain of the adenylate cyclase of B. pertussis, respectively. Different combinations of the GltC and the RocG fusions were coexpressed in the E. coli strain BTH101. If a direct interaction between RocG and GltC occurred, the domains of the adenylate cyclase would get in close proximity leading to the production of cAMP (Karimova et al., 1998). The cAMP production was qualitatively monitored on plates containing IPTG and X-Gal (see Fig. 3.1). Thus, a strong interaction between the respective proteins is reflected by the formation of dark blue colonies on plates. The leucine zipper of the yeast GCN4 served as the positive control in this experiment.

As shown in Fig. 3.1, the GltC as well as the RocG protein tended to perform selfinteraction indicated by blue colored colonies. This result is in good agreement with previous data for the RocG protein. Like many other glutamate dehydrogenases RocG assemblies as a hexamer consisting of two trimers (Gunka et al., 2010). The GltC protein has not been successfully subjected to crystallization until now. But it was shown for other members of the LysR family that they act as tetramers consisting of two dimers (Picossi et al., 2007; Maddocks \& Oyston, 2008). The observed self-interaction of both proteins implies that all fusions were expressed and active in the bacterial twohybrid assay. However, a clear interaction between the RocG and GltC protein could not be observed in this assay. E. coli cells expressing T18-GltC and RocG-T25 were only light-blue colored. As the co-expression of T18-GltC and leucine zipper control T25-zip resulted in an even more intensive blue color, the interaction between T18-GltC and RocG-T25 was defined as non specific. 
Although all used fusions of the RocG and GltC protein with domains of the adenylate cyclase seemed to be expressed and active in this assay, no specific interaction between RocG and GltC was detected. Thus, this method is not appropriate to analyze this protein-protein interaction.

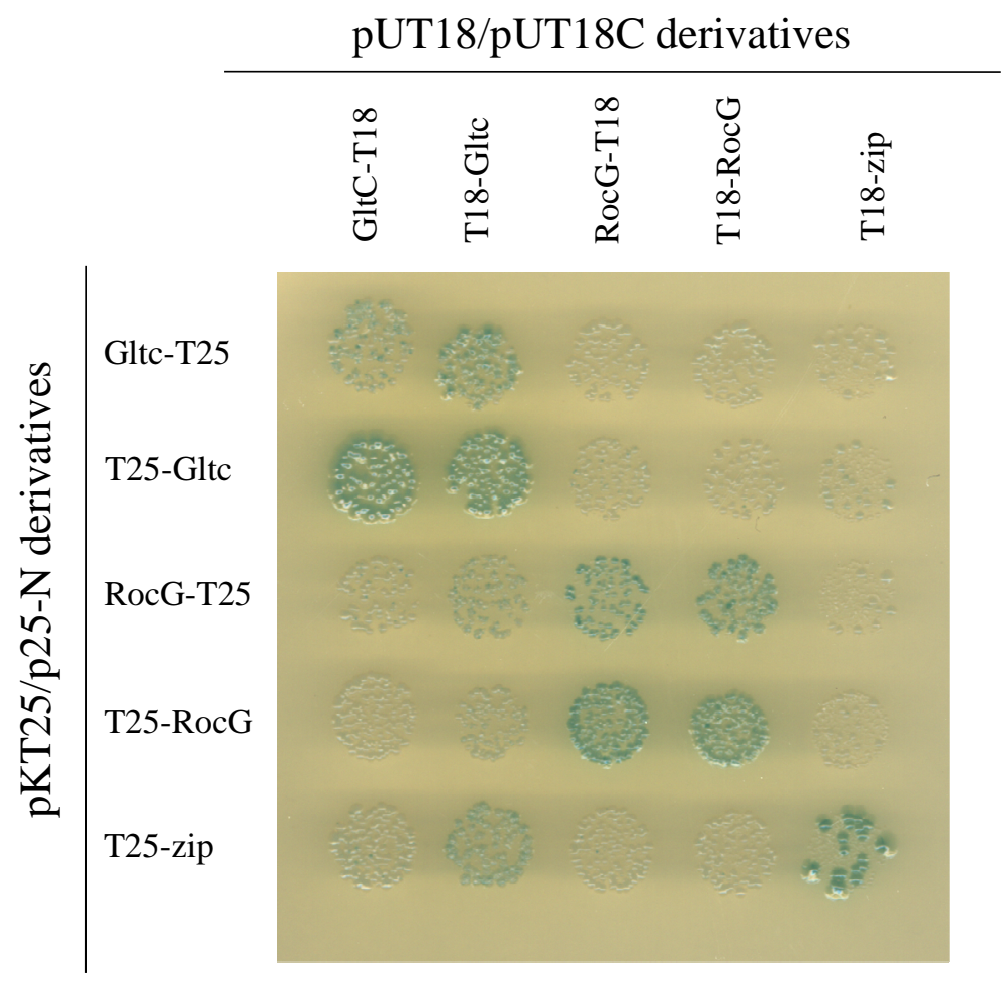

\section{Fig. 3.1 - Bacterial two-hybrid assay of the RocG-GItC interaction.}

Both genes were cloned into the plasmids pUT18, pUT18C, p25-N and pKT25. As illustrated, different combinations of the plasmids were co-expressed in E. coli. The transformants were incubated for $48 \mathrm{~h}$ at $30^{\circ} \mathrm{C}$. Degradation of $\mathrm{X}-\mathrm{Gal}$ (blue color) indicates the formation of a functional adenylate cyclase resulting from the interaction of the fused proteins.

\section{SPINE approach with RocG variants}

The expression of superrepressor RocG (RocG-SR) proteins in B. subtilis results in a strongly abolished gltA-lacZ expression in medium containing glutamate or its precursor arginine. Furthermore, the presence of the monofunctional RocG (RocG-MF) does not repress GltC activity indicated by a high gltA-lacZ expression (Gunka et al., 2010). The gltA-lacZ expression reflects the capacity of the RocG variants to control GltC. However, these data do not provide evidence whether the RocG variants, 
especially the RocG-MF protein, have an altered interaction pattern with the GltC protein in comparison to the wild type RocG.

In order to get a snapshot of the GltC interaction exerted by the mutant RocG variants, a SPINE experiment was performed. In this approach the RocG protein was expressed with a Strep-tag in B. subtilis. By using formaldehyde, the status of the cell was fixed and the RocG protein could be purified via the Strep-tag with its in vivo interaction partners.

The B. subtilis strain GP717 was used in this study. This strain lacks both glutamate dehydrogenase genes and bears a gltA-lacZ fusion to monitor GltC activity. GP717 was transformed with plasmids containing the $\operatorname{roc} G$ wild type, the $\operatorname{roc} G$-SR3 or the rocG-MF allele (Gunka et al., 2010). All alleles were constitutively expressed from the plasmids. The empty vector pGP380 served as the negative control. The strains were grown in $\mathrm{C}$ minimal medium, containing $5 \mathrm{~g} / \mathrm{l}$ glucose and $5 \mathrm{~g} / \mathrm{l}$ arginine, to an $\mathrm{OD}_{600}$ of 1.0. One half was harvested and the other half was treated with $6 \mathrm{~g} / \mathrm{l}$ (final concentration) formaldehyde and incubated for 20 min under agitation and harvested. Prior to the formaldehyde treatment small aliquots were taken for measuring gltA-lacZ activity just in time of the cross-linking. After the Strep-tag purification, the elution fractions were boiled to resolve the cross-linking and separated by SDS-PAGE. Additionally, a Western blot experiment with antibodies raised against the GltC protein was performed (see Fig. 3.2A).

As shown in Fig. 3.2A there was no GltC protein detected in the elution fraction of the empty vector pGP380. After cross-linking with formaldehyde, GltC was detected in the elution fraction of the RocG wild type protein, the superrepressor protein (RocGSR3) and even the monofunctional RocG protein (RocG-MF). This result demonstrates that all used RocG proteins interact with the GltC protein under the condition tested.

Fig. 3.2B shows the gltA-lacZ activity of the strain GP717 transformed with the plasmids as indicated just prior to cross-linking with formaldehyde. As expected, in absence of any RocG protein GltC was fully active (empty vector pGP380) resulting in a high activity of the gltA-lacZ fusion with 443 Miller units. The strain expressing the RocG wild type protein showed a lower gltA-lacZ activity with 56 Miller units. 
(A)

\begin{tabular}{|c|c|}
\multicolumn{1}{c}{-FA $\quad$ +FA } & (Miller Units) \\
\hline $\begin{array}{c}\text { pGP380 } \\
\text { Empty vector }\end{array}$ & 443 \\
\hline $\begin{array}{c}\text { pGP1708 } \\
\text { RocG WT }\end{array}$ & 56 \\
\hline $\begin{array}{c}\text { pGP652 } \\
\text { RocG-SR3 } \\
\text { (E93K) }\end{array}$ & 13 \\
\hline $\begin{array}{c}\text { pGP1709 } \\
\text { RocG-MF } \\
\text { (D122N) }\end{array}$ & 561 \\
\hline
\end{tabular}

Fig. 3.2 - Detection of the RocG-GItC interaction.

(A) $15 \mu \mathrm{l}$ of each elution fraction were separated by a SDS-PAGE. The SDS-PAGE was blotted on a PVDF membrane. For detection of GltC polyclonal antibodies raised against the GltC protein were used. (B) gltA-lacZ expression in crude extracts of the B. subtilis strain GP717 transformed with the respective $\operatorname{roc} G$ alleles cultivated in $\mathrm{C}$ minimal medium with glucose $(5 \mathrm{~g} / \mathrm{l})$ and arginine $(5 \mathrm{~g} / \mathrm{l})$.

The presence of the RocG SR3 protein decreased the GltC activity even stronger than the wild type RocG resulting in only 13 Miller units of the gltA-lacZ fusion. This is in line with previously results (Gunka et al., 2010). Due to these low gltA-lacZ activities it was tempting to speculate that the RocG wild type and SR3 protein interact with GltC under this condition. The presence of the RocG MF protein does not lead to an inhibition of the GltC activity. The gltA-lacZ fusion is highly expressed with 561 Miller units. However, this abolished inhibition cannot be explained by an eliminated interaction of RocG MF with GltC as shown in Fig. 3.2A. This result suggests that the pure physical interaction between RocG and GltC is not sufficient for GltC inactivation. Actually, the RocG MF protein still interacts with GltC but is not capable of exerting a negative effect on the GltC activity anymore. 
Mutant RocG variants have no impact on the GItC protein level

In order to exclude that the effect of the mutant RocG variants on gltA-lacZ expression is based on an altered GltC protein level, the GltC expression was analyzed in a Western blot experiment (see Fig. 3.3).

\section{GltC}

\begin{tabular}{ccccccc}
\hline SR1 & SR2 & SR3 & SR5 & MF & WT & \\
I308T & S234R & E93K & Y158H & D122N & & Empty vector \\
& & & & & & \\
& & & & & & \\
& & & & & & \\
\end{tabular}

Fig. 3.3 - Expression of GltC in the context of RocG mutant proteins.

Crude extracts were isolated from B. subtilis strain GP28 (rocG $\Delta g u d B$ ) expressing the RocG superrepressor (SR) proteins SR1, SR2, SR3, and SR5, and the monofunctional (MF) RocG protein grown in CSE minimal medium. Strain GP28 either expressing the RocG wild-type (WT) protein or carrying the empty vector pBQ200 served as control to compare GltC protein level. GltC was detected using rabbit polyclonal antibodies raised against B. subtilis GltC. Samples (15 $\mu \mathrm{g})$ of crude extract were applied per lane.

As shown in Fig. 3.3 the expression of the mutant RocG variants had no impact on the GltC expression. Therefore, it can be assumed that the effect of these RocG variants on $\operatorname{glt} A B$ expression is not linked to an altered GltC protein level. 


\section{Discussion}

To the best of our knowledge, every method to reconstruct the RocG-GltC interaction in vitro has failed until now. Although the fusions of RocG and GltC protein seemed to be expressed and active in the bacterial two-hybrid assay as indicated by selfinteractions, no specific interaction between RocG and GltC was detected. Even though it is accepted that every individual method to study protein-protein interactions can detect only about one third of the actual interactions (Braun et al., 2009), the question remains why also approaches like SPR have failed in showing the interaction (J. A. Newman., pers. comm.) The most obvious explanation why the bacterial two-hybrid assay was not successful in showing the RocG-GltC interaction is that a factor is missing in E. coli that is present in B. subtilis. In principle, there are different classes of factors possible that facilitate protein-protein interactions.

First, low-molecular weight factors are known to be involved in a particular interaction. A well studied example is the glutamine synthetase in B. subtilis. In the presence of glutamine the glutamine synthetase interacts with the transcription regulator TnrA inhibiting the DNA binding activity of TnrA (Wray et al., 2001). Moreover, the glutamine synthetase also regulates the activity of another important transcription regulator of nitrogen metabolism, GlnR, in a glutamine-dependent manner (Fisher \& Wray, 2008). In analogy to this regulation it can be hypothesized that the interaction between RocG and GltC is triggered by glutamate as this amino acid is the substrate of the glutamate dehydrogenase RocG and can therefore by easily sensed by RocG. This idea is supported by the observation that RocG can only repress GltC activity in the presence of glutamate or its precursor arginine (Gunka et al., 2010). But as it was demonstrated that glutamate is one of the most abundant metabolites also in E. coli (Bennett et al., 2009) it is unlikely that glutamate is limiting in the bacterial two-hybrid assay. Thus, the availability of glutamate cannot be the only requirement for the RocGGltC interaction.

The second possibility is that a third protein might be involved in the formation of the RocG-GltC complex. But if the interaction of RocG with GltC is not direct but mediated by another protein we would have expected to find an additional interaction partner in a SPINE approach in relevant amounts (Commichau et al., 2007a). However, this possibility cannot be completely excluded. 
Third, an obvious difference between the bacterial two-hybrid assay compared to the situation in $B$. subtilis is the presence of the gltAB promoter region that is the target of GltC binding. In this promoter region GltC can bind to multiple locations (Bohannon \& Sonenshein, 1989; Belitsky et al., 1995; Picossi et al., 2007). The ratio of 2-oxoglutarate to glutamate is supposed to regulate GltC binding capacity to the different locations. At high 2-oxoglutarate concentration GltC activates the gltABexpression whereas at higher glutamate concentrations the GltC conformation is altered and the expression of the gltAB genes is not activated (Picossi et al., 2007). In this complex model of regulation it is tempting to speculate that RocG modulates the GltC activity by binding to $\mathrm{GltC}$ which has to be associated with its target promoter region. Perhaps GltC does not assemble in such a conformation that can be bound by RocG until it is located at its promoter region. This hypothesis would explain why the RocGGltC interaction cannot be detected in the bacterial two-hybrid assay.

Moreover, the cross-linking experiment with the mutant RocG protein implies that interaction is not the equivalent of inhibition. It was demonstrated that all RocG proteins interact with GltC, although the RocG-MF lacks the ability to repress GltC activity. This result supports the idea that the RocG-GltC interaction is a very complex regulation mechanism. The interaction between RocG and GltC seems to have different effects on the GltC activity. Perhaps the effect is influenced by the presence of metabolites e.g. glutamate and 2-oxoglutarate. Furthermore, it was demonstrated that the RocG mutant variants specifically influence the GltC activity in terms of gltAB transcription activation and not the autoregulative properties of GltC. To get a more precise insight into the interaction mechanism, this topic needs to be subject or further investigation.

\section{Future perspectives}

As discussed above, alternative evidence to the SPINE experiment for the RocGGltC interaction is still missing. To test the hypothesis, whether the protein-protein interaction between RocG and GltC depends on the presence of the target promoter region of the transcription activator GltC, the defined DNA sequence can be introduced in the background of the bacterial two-hybrid assay. This can be simply achieved by cloning the promoter region into one of the vectors for the RocG and GltC coexpression. If the gltAB promoter is necessary to facilitate the right conformation of 
GltC and enables RocG to interact with GltC, this interaction should be detected in the bacterial two-hybrid assay in the presence of the promoter sequence.

Furthermore, a SPINE experiment in the absence of glutamate or a source of it can provide more insights into the mechanism of $\mathrm{GltC}$ regulation by RocG. If RocG also interacts with GltC in the absence of glutamate this would support the idea that the impact of the interaction on GltC activity depends on other factors e.g. the ratio glutamate to 2-oxoglutarate. A barrier in performing this experiment is that the RocG protein is naturally not expressed under this condition (Belitsky \& Sonenshein 1999). In order to circumvent this problem RocG could be constitutively expressed from a plasmid and serve as the bait to catch GltC. Another opportunity to test the interaction in the absence of glutamate is to express GltC with a Strep-tag in a B. subtilis gudBI mutant strain. The active glutamate dehydrogenase GudB1 was shown to interact with GltC and is expressed under this condition (Commichau et al., 2007a, Gunka et al., 2010 subm.). 


\section{Chapter 4}

A high-frequency mutation in Bacillus subtilis: Requirements for the decryptification of the $\operatorname{gudB}$ glutamate dehydrogenase gene

The work described in this chapter was submitted for publication in:

Gunka, K., Tholen, S. Neme, R., Freytag, B., Commichau, F. M., Herzberg, C. \&

Stülke, J. A high-frequency mutation in Bacillus subtilis: Requirements for the decryptification of the gudB glutamate dehydrogenase gene. Mol. Microbiol. submitted

Author contributions: This study was designed and interpreted by KG, ST, FMC and JS. KG and ST performed most of the experiments. RNG constructed several mutant strains and performed Northern Blot experiments. BF contributed to this study by generating some mutant strains. FMC helped to develop the artificial mutation system and $\mathrm{CH}$ was essential to improve the method part. KG and JS wrote the paper. 


\begin{abstract}
Bacillus subtilis encodes two glutamate dehydrogenases, the enzymatically active protein RocG and the cryptic enzyme GudB that is inactive due to a duplication of three amino acids in its active centre. The inactivation of the $\operatorname{roc} G$ gene results in poor growth of the bacteria on complex media likely due to the accumulation of toxic intermediates. Therefore, $\operatorname{roc} G$ mutants readily acquire suppressor mutations that decryptify the $g u d B$ gene. This decryptification occurs by a precise deletion of one part of the nine base pair direct repeat that causes the amino acid duplication. This mutation occurs at the extremely high rate of $10^{-4}$. Mutations affecting the integrity of the direct repeat result in a strong reduction of the mutation rate; however, the actual sequence of the repeat is not essential. The mutation rate of $g u d B$ was not affected by the position of the gene on the chromosome. When the direct repeat was placed in the completely different context of an artificial promoter, the precise deletion of one part of the repeat was also observed, but the mutation rate was reduced by three orders of magnitude. Thus, transcription of the $\operatorname{gudB}$ gene seems to be essential for the high rate of the appearance of the $g u d B l$ mutation. This idea is supported by the finding that the transcription-repair coupling factor Mfd is required for the decryptification of $g u d B$. The Mfd-mediated coupling of transcription to mutagenesis can be regarded as a built-in precaution that facilitates the accumulation of mutations preferentially in transcribed genes.
\end{abstract}

\title{
Introduction
}

As the central amino group donor for nearly all biosynthetic pathways in any living cell, glutamate plays a key role in the biochemistry and physiology of all organisms (Commichau et al., 2006). Investigations with Escherichia coli demonstrate that glutamate is by far the most abundant metabolite in these bacteria accounting for about $40 \%$ of the internal metabolite pool (Yuan et al., 2009). Moreover, glutamate is one of the most highly embedded metabolites: In the Gram-positive soil bacterium Bacillus subtilis, at least 37 reactions make use of this amino acid (Oh et al., 2007).

In $B$. subtilis, glutamate is exclusively synthesized from 2-oxoglutarate and glutamine by the activity of glutamate synthase. 2-Oxoglutarate is replenished in the 
citric acid cycle, whereas glutamine can be synthesized with ammonium as the nitrogen source and one of the two molecules of glutamate that are generated by glutamate synthase as the acceptor. Glutamate does also serve as a precursor for proline biosynthesis, and under conditions of osmotic stress, molar concentrations of proline have to be produced (Kempf \& Bremer, 1998). Thus, it is not surprising that glutamate synthesis has to be a highly efficient process, and indeed, interactions between enzymes of the branch of the citric acid cycle that generates 2-oxoglutarate and glutamate synthase have been reported (Meyer et al., 2011). Glutamate can also serve as source of carbon and nitrogen. Its utilization is initiated by an oxidative deamination catalyzed by the glutamate dehydrogenase. The expression of the genes encoding glutamate biosynthetic and catabolic enzymes is subject to complex control mechanisms that allow the adjustment of the intracellular glutamate concentration to the actual requirement (Belitsky \& Sonenshein, 1999; Belitsky et al., 2004; Commichau et al., 2007b; Picossi et al., 2007; Sonenshein, 2007).

B. subtilis encodes two glutamate dehydrogenases, GudB and RocG (Belitsky \& Sonenshein, 1998). However, the gudB gene experienced an inactivating mutation during domestication, resulting in an inactive pseudogene in the laboratory strain $B$. subtilis 168. In contrast, the $g u d B$ gene encodes an active enzyme in wild isolates and in non-domesticated strains such as NCIB3610 (Zeigler et al., 2008). The inactivation of $\operatorname{gudB}$ is caused by a duplication of nine base pairs of the coding sequence resulting in a duplication of three amino acids in the active centre of the protein. The glutamate dehydrogenase RocG catalyzes the final step of the catabolic pathway for arginine, ornithine and citrulline. Accordingly, its expression is strongly induced in the presence of arginine (Belitsky \& Sonenshein, 1998). Interestingly, the glutamate dehydrogenases are not only required for glutamate utilization, but they are also involved in the control of glutamate biosynthesis: In the presence of glutamate they inhibit the transcription activator GltC that is necessary for the expression of the glutamate synthase operon, gltAB (Commichau et al., 2007a; Commichau \& Stülke, 2008; Gunka et al., 2010). In the active state, the two glutamate dehydrogenases are very similar to each other, both at the level of the amino acid sequence and also concerning their structures. In contrast, the inactive GudB protein seems to misfold and is subject to rapid degradation (Gerth et al., 2008; Gunka et al., 2010). 
The importance of glutamate for the cellular physiology is underlined by the observation that any mutation that disturbs the glutamate homoeostasis results in the accumulation of suppressing mutations. This is true for both E. coli and B. subtilis (Yan, 2007; Commichau et al., 2008). In the laboratory strain of B. subtilis, the inactivation of the $\operatorname{roc} G$ gene encoding the only active glutamate dehydrogenase results in the appearance of mutants with an active GudB enzyme (these alleles are designated GudB1) (Belitsky \& Sonenshein, 1998). Moreover, rocG gudB double mutants easily acquire suppressive mutations affecting the glutamate synthase (Commichau et al., 2008). The $\operatorname{roc} G \operatorname{gudB}$ double mutants are unable to utilize glutamate as the source of carbon and nitrogen. However, cultivation of such mutants in the presence of glutamate or its precursors such as arginine results in the selection of suppressor mutants that catabolize glutamate by a pathway that is not operative in wild type bacteria. The analysis of one such mutant revealed constitutive expression of the aspartase pathway due to the inactivation of the repressor of the corresponding ans $A B$ operon, AnsR (Flórez et al., subm.).

The accumulation of mutations that restore growth of mutants or that allow faster growth is a common phenomenon in bacteria. Several studies suggest that mutations that overcome the specific limitation are preferentially acquired (Cairns et al., 1988; Barrick et al., 2009); however, the underlying mechanisms have not yet been elucidated.

Mutations can be acquired during replication. Most of the errors are eliminated by DNA mismatch repair, including the MutSL system which contributes to genome stability (Modrich \& Lahue, 1996; Fukui, 2010). Some errors can escape from repair and may be beneficial for the organism. Many bacteria including B. subtilis possess systems for the induction of mutations in the stationary phase (Sung \& Yasbin, 2002). The emergence of these mutations is associated with transcription rather than with DNA replication and plays an important role in the generation of diversity in nondividing populations of $B$. subtilis. The process of transcription-coupled DNA repair is crucial for the accumulation of mutations in the stationary phase, and this involves the transcription repair coupling factor Mfd (Ayora et al., 1996). The Mfd protein targets DNA lesions during transcription that provoked a roadblock of transcription. Subsequently, Mfd may displace the RNA polymerase and recruit the nucleotide excision repair system to resolve the lesion (Borukhov et al., 2005; Truglio et al., 2006). 
It was suggested that this process favors the acquisition of beneficial mutations of highly transcribed genes (Ross et al., 2006; Pybus et al., 2010).

We are interested in the mechanism by which the decryptification of the $\operatorname{gudB}$ gene occurs in $\operatorname{roc} G$ mutants. The gudBl mutation appears during growth and requires a deletion of nine base pairs. Therefore, $g u d B$ provides a unique system to study the emergence of mutations. Our results suggest that the decryptification of $\operatorname{gudB}$ requires the presence of a perfect direct repeat. Moreover, a part of this repeat is only deleted in the context of a transcribed gene, and this deletion requires the Mfd transcription repair coupling factor. 


\section{Materials and Methods}

\section{Bacterial strains and growth conditions}

All B. subtilis strains used in this work are derived from the laboratory wild type strain 168. They are listed in Tab. S3. E. coli DH5 $\alpha$ (Sambrook et al., 1989) was used for cloning experiments. B. subtilis was grown in SP medium, LB medium or in C minimal medium supplemented with tryptophan (at $50 \mathrm{mg} / \mathrm{l}$ ) (Wacker et al., 2003). CSE medium is $\mathrm{C}$ minimal medium supplemented with sodium succinate $(6 \mathrm{~g} / \mathrm{l})$ and potassium glutamate $(8 \mathrm{~g} / \mathrm{l})$. C-Glc is $\mathrm{C}$ minimal medium supplemented with glucose (5 $\mathrm{g} / \mathrm{l})$, and CS is supplemented with sodium succinate (6 g/l) (Wacker et al., 2003). Additional sources of carbon and nitrogen were added as indicated. E. coli was grown in LB medium and transformants were selected on plates containing ampicillin (100 $\mu \mathrm{g} / \mathrm{ml}$ ). LB, SP and CS plates were prepared by the addition of $17 \mathrm{~g}$ Bacto agar/l (Difco, Lawrence, USA) to LB, SP or CS medium, respectively.

\section{DNA manipulation, transformation and phenotypic analysis}

Transformation of E. coli and plasmid DNA extraction were performed using standard procedures (Sambrook et al., 1989). Restriction enzymes, T4 DNA ligase and DNA polymerases were used as recommended by the manufacturers. DNA fragments were purified using the QIAquick PCR Purification kit (Qiagen, Hilden, Germany) Phusion $^{\mathrm{TM}}$ DNA polymerase (New England Biolabs, Frankfurt am Main, Germany) was used for the polymerase chain reaction as recommended by the manufacturer. All primer sequences are provided as supplementary material (Tab. S1). DNA sequences were determined using the dideoxy chain termination method (Sambrook et al., 1989). All plasmid inserts derived from PCR products were verified by DNA sequencing. Chromosomal DNA of B. subtilis was isolated as described (Kunst \& Rapoport, 1995).

E. coli transformants were selected on LB plates containing ampicillin (100 $\mu \mathrm{g} / \mathrm{ml})$. B. subtilis was transformed with plasmid or chromosomal DNA according to the two-step protocol described previously (Kunst \& Rapoport, 1995). Transformants were selected on SP plates containing kanamycin (Kan $10 \mu \mathrm{g} / \mathrm{ml})$, chloramphenicol $(\mathrm{Cm} 5 \mu \mathrm{g} / \mathrm{ml}$ ) spectinomycin (Spec $150 \mu \mathrm{g} / \mathrm{ml}$ ) or erythromycin plus lincomycin (Erm 2 $\mu \mathrm{g} / \mathrm{ml}$ and $\mathrm{Lin} 25 \mu \mathrm{g} / \mathrm{ml})$. 
In $B$. subtilis, amylase activity was detected after growth on plates containing nutrient broth (7.5 g/l), $17 \mathrm{~g}$ Bacto agar/l (Difco, Lawrence, USA) and 5 g hydrolyzed starch/l (Merck, Darmstadt, Germany). Starch degradation was detected by sublimating iodine onto the plates.

Quantitative studies of lacZ expression in B. subtilis were performed as follows: cells were grown in CSE medium supplemented with different carbon and nitrogen sources as indicated. Cells were harvested at $\mathrm{OD}_{600}$ of 0.6 to 0.8 for cultures in CSE medium and 0.8 to 1.0 for cultures in CSE medium with sugar. $\beta$-Galactosidase specific activities were determined with cell extracts obtained by lysozyme treatment as described previously (Kunst \& Rapoport, 1995). One unit of $\beta$-galactosidase is defined as the amount of enzyme which produces $1 \mathrm{nmol}$ of o-nitrophenol per min at $28^{\circ} \mathrm{C}$.

\section{Ectopic expression of gudB variants}

To express the gudB gene at an ectopic site, we used plasmid pAC5 (Martin-Verstraete et al., 1992). This plasmid allows integration of the cloned fragments into the amyE site of the B. subtilis chromosome. Briefly, the gudB gene was amplified with its natural promoter using the oligonucleotides ST1 and KG92 using chromosomal DNA of B. subtilis 168 as the template. The PCR product was digested with EcoRI and BamHI and cloned into pAC5 linearized with the same enzymes. The resulting plasmid pGP900 was used to introduce the $\operatorname{gudB}$ allele into the chromosome.

The direct repeat of $g u d B$ present in pGP900 was subjected to site-directed mutagenesis by a modified PCR protocol, the combined chain reaction (Bi \& Stambrook, 1998). Primers ST1 and KG92 were used as outer primers. The primers $\mathrm{KG} 119$, KG120, and KG133 were used to introduce point mutations into the gudB coding region. These primers were phosphorylated at their 5' end and allowed ligation of the nascent elongation product initiated from ST1. The resulting products carrying the mutations were cut with EcoRI and BamHI and cloned into pAC5 digested with the same enzymes. The resulting plasmids were pGP1714 (G3T G9T), pGP1715 (G3T G9T G12T G18T), and pGP1721 (G12T G18T). The plasmids were linearized with PstI and used to transform B. subtilis (see Tab. S2). 


\section{Design and construction of a mutagenesis reporter system}

In order to analyze the occurrence of the deletion of the repeat in a non-related sequence context, we developed a reporter system which is based on a promoter that is only active upon deletion of one part of the $g u d B$-derived direct repeat. This artificial alf promoter controls the expression of genes coding for a kanamycin resistant determinant (aphA3) and E. coli $\beta$-galactosidase (see Fig. 4.2). To obtain the reporter strain, we first constructed plasmid pGP655 as follows: The promoterless aphA3 gene was amplified from pDG780 (Guérot-Fleury et al., 1995) using the primer pair ST4/ST9. These oligonucleotides attached restriction sites for EcoRI and BamHI (ST9) and for BglII (ST4) to the PCR product. The fragment was digested with EcoRI and BglII and cloned into the integration vector pAC6 (Stülke et al., 1997) linearized with EcoRI and BamHI. The resulting plasmid pGP653 contained a promoterless aphA3-lacZ operon. The alf promoter fragment was obtained by hybridization of the complementary oligonucleotides ST7 and ST8. It was cloned between the EcoRI and BamHI sites of pGP653, resulting in plasmid pGP655.

\section{Construction of mutant strains}

The $\Delta g u d B$ and $\Delta m f d$ mutant strains were obtained by applying the long flanking homology PCR (LFH-PCR) technique (Wach et al., 1996). For the construction of the $\triangle \operatorname{gudB}$ mutant, an aphA3 resistance cassette was first amplified from plasmid pDG780 using the primer pair kan-fwd/kan-rev (Guérot-Fleury et al., 1995). DNA fragments of about $1,000 \mathrm{bp}$ flanking the $g u d B$ region at its 5 ' and 3 ' end were amplified using the primer pairs KG100/KG101 and KG102/KG103. Chromosomal DNA of B. subtilis 168 served as the template. The 3 ' end of the upstream fragment as well as the 5' end of the downstream fragments extended into the respective gene region, in a way that all expression signals of genes up- and downstream remained intact. The joining of the two fragments to the resistance cassette was performed in a second PCR. In this reaction we used the primer pair KG100/KG103 for the deletion of $\operatorname{gudB}$. The PCR product was directly used to transform $B$. subtilis 168 . The integrity of the regions flanking the integrated resistance cassette was verified by sequencing PCR products of about 1,000 bp amplified from chromosomal DNA of the resulting mutant GP1160. 
The $\Delta m f d$ mutant GP1167 was obtained in a similar way. Briefly, the ermC resistance gene was amplified from pDG647 with the primers mls-fwd(kan)/mls$\operatorname{rev}(k a n)$ (Guérot-Fleury et al., 1995), and the regions flanking $m f d$ were amplified using the primer pairs KG86/KG87 and KG88/KG89. The joining PCR was performed with the primer pairs KG86/KG89. The PCR product was used to transform B. subtilis 168. As described above, the integrity of the DNA fragments flanking the resistance cassette in GP1167 was verified by sequencing.

\section{Construction of a gudB-lacZ fusion}

To determine the activity of the $g u d B$ promoter, a translational fusion of the $g u d B$ promoter to a promoterless lac $Z$ gene encoding $\beta$-galactosidase was constructed as follows. A DNA fragment containing the $\operatorname{gudB}$ promoter region was generated by PCR using the primers ST1 and ST2, digested with BamHI and EcoRI and cloned into the plasmid pAC5. The plasmid pAC5 contains a promoterless lacZ gene and allows the introduction of translational fusions into the $a m y E$ locus of $B$. subtilis (Martin-Verstraete et al., 1992). The resulting plasmid pGP651 was used to introduce the fusion into different B. subtilis mutants (see Tab. S3).

\section{Determination of mutation rates}

All $\operatorname{roc} G$ mutant strains were plated on CS medium to verify the presence of the cryptic gudB wild type allele in the respective strains. The roc $G$ mutants were able to grow on this medium as long as the $g u d B$ gene had remained inactive (Commichau et al., 2007b). Mutation rates were determined by the method of the median (Lea \& Coulson, 1949). Briefly, eleven cultures in CSE-Glc were inoculated to a density of 100 cells/ml with an overnight culture grown in the same medium. The cultures were incubated at $37^{\circ} \mathrm{C}$ to an $\mathrm{OD}_{600}$ of 2.0 . For the analysis of culture titers appropriate dilutions of four cultures were plated on SP medium containing glucose to allow growth of the $\operatorname{roc} G$ mutant strains. To screen for $g u d B 1$ mutations, appropriate dilutions of each culture were plated on SP medium. After $24 \mathrm{~h}$ colonies showing the gudB1 phenotype (wild type-like colonies on SP plates) were counted. To be sure of the identity of the mutations, the $g u d B$ allele was sequenced for at least three independent suppressor mutants in each experiment. In every single case, the correct excision of one part of the 
repeat (i.e. the gudB1 mutation) was observed. For the determination of mutation rates of the alf promoter present in the strains GP1123 and GP1168, the bacteria were plated on SP medium containing kanamycin $(60 \mu \mathrm{g} / \mathrm{ml})$ and X-Gal $(80 \mu \mathrm{g} / \mathrm{ml})$.

\section{Northern blot analysis}

Preparation of total RNA and Northern blot analysis were carried out as described previously (Ludwig et al., 2001). Digoxigenin (DIG) RNA probes were obtained by in vitro transcription with T7 RNA polymerase (Roche Diagnostics, Basel, Switzerland) using PCR-generated DNA fragments as templates. The primer pairs used to amplify DNA fragments specific for $g u d B$ and gapA are listed in Tab. S1. The reverse primers contained a T7 RNA polymerase recognition sequence. In vitro RNA labelling, hybridization and signal detection were carried out according to the instructions of the manufacturer (DIG RNA labelling kit and detection chemicals; Roche Diagnostics, Basel, Switzerland). To determine the size of the $\operatorname{gudB}$ mRNA, we used the transcripts observed with a gapA probe as the standard. RNA stability was analyzed as described previously (Meinken et al., 2003). Briefly, rifampicin was added to logarithmically growing cultures (final concentration $100 \mu \mathrm{g} / \mathrm{ml}$ ) and samples were taken at the time points indicated. The quantification was performed using the ImageJ software v1.42 (Abramoff et al., 2004).

\section{Western blotting}

For Western blot analysis, proteins were separated by $12.5 \%$ SDS-PAGE and transferred onto polyvinylidene difluoride (PVDF) membranes (Bio-Rad, Munich, Germany) by electroblotting. Rabbit anti-RocG (1:15,000) (Commichau et al., 2007b) served as the primary antibody. The antibodies were visualised by using anti-rabbit immunoglobulin G-alkaline phosphatase secondary antibodies (Promega, Madison, USA) and the CDP* detection system (Roche Diagnostics, Basel, Switzerland), as described previously (Commichau et al., 2007a). 


\section{Results}

\section{The gudB1 suppressor mutation appears at an extremely high rate}

In our previous studies we observed that the $g u d B$ gene readily acquired the gudB1 suppressor mutation if the rocG gene was inactivated (Commichau et al., 2007b; Commichau et al., 2008). In order to describe this mutation event in a quantitative way, the rate of the $g u d B$ reversion was determined. For this purpose, eleven independent cultures of the $\operatorname{roc} G$ mutant strain $B$. subtilis GP747 were inoculated with approximately 100 cells $/ \mathrm{ml}$ to reduce the likelyhood of very early mutants. The cultures were grown under non-selective conditions (in CSE medium supplemented with glucose) for 20 generations and plated on complex medium (SP medium) which is toxic for the $\operatorname{roc} G$ mutant but not for emerging $\operatorname{roc} G$ gudBl suppressor strains. Suppressor mutants were recognized since they grew as solid colonies (like the wild type strain $B$. subtilis 168) whereas the rocG mutant strain GP747 formed only very small opaque colonies on complex medium. To ascertain that the mutation had appeared during the cultivation and not as a result of selection on the plates, only suppressor mutants that were present after 24 hours were taken into consideration. The mutation rate was $1 \mathrm{x}$ $10^{-4}$. To the best of our knowledge, such a high mutation rate has never been observed before in $B$. subtilis.

\section{The role of chromosomal location and the direct repeat for the high reversion rate of gudB}

The extremely high rate of reversion of $g u d B$ might result from the presence of a direct repeat of nine base pairs. However, the chromosomal arrangement might play a role as well. To distinguish between these possibilities, we decided to address the role of the chromosomal location of the $\operatorname{gudB}$ gene first. For this purpose, we used the B. subtilis strain GP1163. In this strain, the chromosomal gudB gene was deleted and another copy of $\operatorname{gudB}$ under the control of its own promoter was inserted ectopically at the amyE site of the chromosome. The mutation rate of this strain was $0.49 \times 10^{-4}$. Sequence analysis of three randomly selected suppressor mutants revealed that all contained the gudB1 mutation. This observation suggests that the chromosomal location has no major impact on the occurrence of the $g u d B 1$ suppressor mutation (see Fig. 4.1A). 
The direct repeat in $g u d B$ is a common feature irrespective of the chromosomal location of the $g u d B$ allele. Therefore, we attempted to address the role of this repeat by a mutation analysis. Since the direct repeat is located within the coding sequence of $\operatorname{gudB}$, any mutation to be introduced into the direct repeat had to conserve the $\operatorname{gudB}$ open reading frame. In order to destroy the direct repeat, we replaced two $\mathrm{G}$ residues by $\mathrm{T}$ (position 3 and 9 of the repeat, corresponding to wobble bases of the codons for valine and alanine). This mutation was introduced into both the first and the second part of the direct repeat; the corresponding strains are B. subtilis GP1179 and GP1197, respectively. Moreover, we restored a direct repeat; albeit with a sequence that deviates from the original repeat by introducing the same mutations in both parts of the repeat. This strain was GP1180 (see Fig. 4.1A).

A

$$
168(\operatorname{gudB})
$$$$
168(\operatorname{gudB1})
$$

GP1179 (gudB $\left.B_{\text {mut }}\right)$

GP1180 (gudB $\left.B_{\text {mut2 }}\right)$ GP1197 (gudB $\left.B_{\text {mut3 }}\right)$

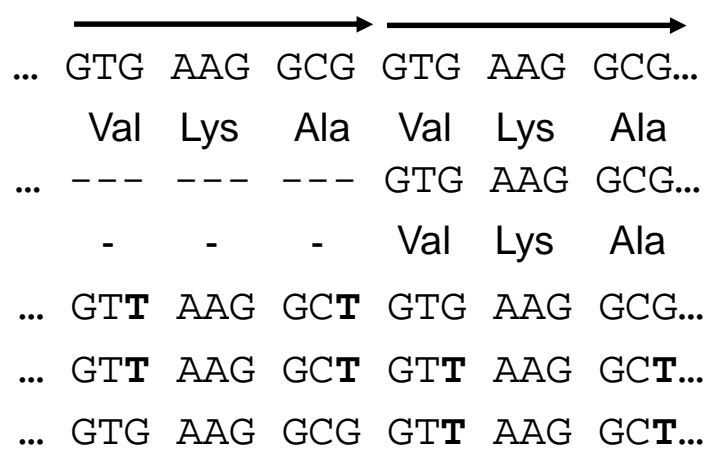

B

\begin{tabular}{|c|c|c|c|c|c|c|c|}
\hline GP1179 (gudB $\left.B_{\text {mut }}\right)$ & ... & --- & $\begin{array}{c}--- \\
-\end{array}$ & $\begin{array}{c}--- \\
-\end{array}$ & $\begin{array}{c}\text { GTG } \\
\text { Val }\end{array}$ & $\begin{array}{l}\text { AAG } \\
\text { Lys }\end{array}$ & $\begin{array}{c}\text { GCG... } \\
\text { Ala }\end{array}$ \\
\hline GP1197 (gudB1 $1_{\text {mut3 }}$ ) & $\ldots$ & --- & --- & --- & GTT & AAG & GCT... \\
\hline & & - & - & - & Val & Lys & Ala \\
\hline GP1197 (gudB1 $1_{\text {mut3 }}$ ) & $\ldots$ & GTG & --- & --- & --- & AAG & GCT... \\
\hline & & Val & - & - & & Lys & Ala \\
\hline
\end{tabular}

Fig. 4.1 - The crucial role of the direct repeat for the decryptification of gudB.

(A) The wild type gudB sequence was mutated without changing the amino acid sequence. In GP1179 two $G$ residues were replaced by $T$ in the first half of the repeat (position 3 and 9 of the repeat). In GP1197 theses mutations were introduced in the second part of the direct repeat (position 12 and 18). The perfect direct repeat was restored in the strain GP1180. This study served to analyze the role of a perfect direct repeat in the rapid decryptification of the $g u d B$ allele. (B) Destroying of the direct repeat leads to a 15 -fold decrease of mutation frequency. In all gudB1 mutants derived from the strain GP1179, the first part of the imperfect repeat was excised. In nine out of 14 gudB1 mutants derived from the strain GP1197 also the first half of the imperfect repeat was deleted whereas in five gudB1 mutants an internal excision had occurred. 
A comparison of the mutation rates revealed that the perfect repeat was a prerequisite for efficient accumulation of $g u d B 1$ suppressor mutants. In the absence of a perfect direct repeat, the mutation rate was reduced by a factor of about $15\left(0.036 \times 10^{-4}\right.$ and $0.034 \times 10^{-4}$ for GP1179 and GP1197, respectively vs. $0.49 \times 10^{-4}$ strain GP1163 carrying the wild type repeat). The introduction of compensatory mutations that restore the direct repeat did also restore the high rate of the appearance of the $g u d B 1$ mutation $\left(0.89 \times 10^{-4}\right.$ for GP1180). These results clearly demonstrate that the presence of the direct repeat is the decisive factor for the high $g u d B 1$ mutation rate.

\section{Selective excision of the first part of the direct repeat}

In all experiments to determine mutation rates, we analyzed the nucleotide sequence of the $g u d B$ suppressor mutations. As stated above, a precise deletion of the direct repeat was observed in all cases. However, the presence of a perfect repeat precluded the identification of the nucleotides that had actually been excised. This question became tractable with the availability of the suppressor mutants of B. subtilis GP1179 and GP1197 in which the repeat is not perfect. The sequence analysis of the gudB1 alleles of eleven suppressor mutants derived from GP1179 (mutated in the first part of the repeat) revealed that the first half of the repeat was deleted in all cases (see Fig. 4.1B). This strong bias might indicate that either the first part of the repeat is preferentially excised or that the naturally occurring sequence is retained with preference. This question was addressed by the analysis of suppressor mutants derived from GP1197 (mutations in the second part of the repeat). In this case, of 14 analyzed mutants, nine had a deletion of the first part of the repeat. Moreover, five mutants exhibited internal deletions of the repeat that restored a sequence coding for the active GudB protein (see Fig. 4.1B). Thus, none of the mutants derived from GP1197 restored the original nucleotide sequence of the remainder of the repeat. Instead, we observed again a strong bias towards deletion of the first part of the repeat suggesting that this selective deletion is inherent to the mutagenesis process that decryptifies the $g u d B$ gene. 


\section{Construction and analysis of a deletion reporter system}

The results presented above demonstrate that the deletion of one part of the $g u d B$ repeat occurs at a very high frequency both in the native and in a non-related genomic context as long as the repeat is intact. These findings prompted us to ask whether the deletion would also take place as efficiently in a completely different sequence context as it does in the $\operatorname{gudB}$ gene. For this purpose, we constructed a reporter system consisting of an aphA3-lacZ operon encoding a resistance to kanamycin and $\beta$-galactosidase under the control of an artificial (alf) promoter. This promoter was designed to have perfect recognition sequences for the housekeeping sigma factor of the RNA polymerase (-10 and -35$)$, however the spacing between the two boxes was 26 rather than the canonical 17 to 18 base pairs. The perfect repeat of the gudB gene should be a part of this spacer. This promoter is not likely to be recognized by the RNA polymerase unless one part of the repeat is deleted and the optimal $17 \mathrm{bp}$ spacing is restored (see Fig. 4.2).
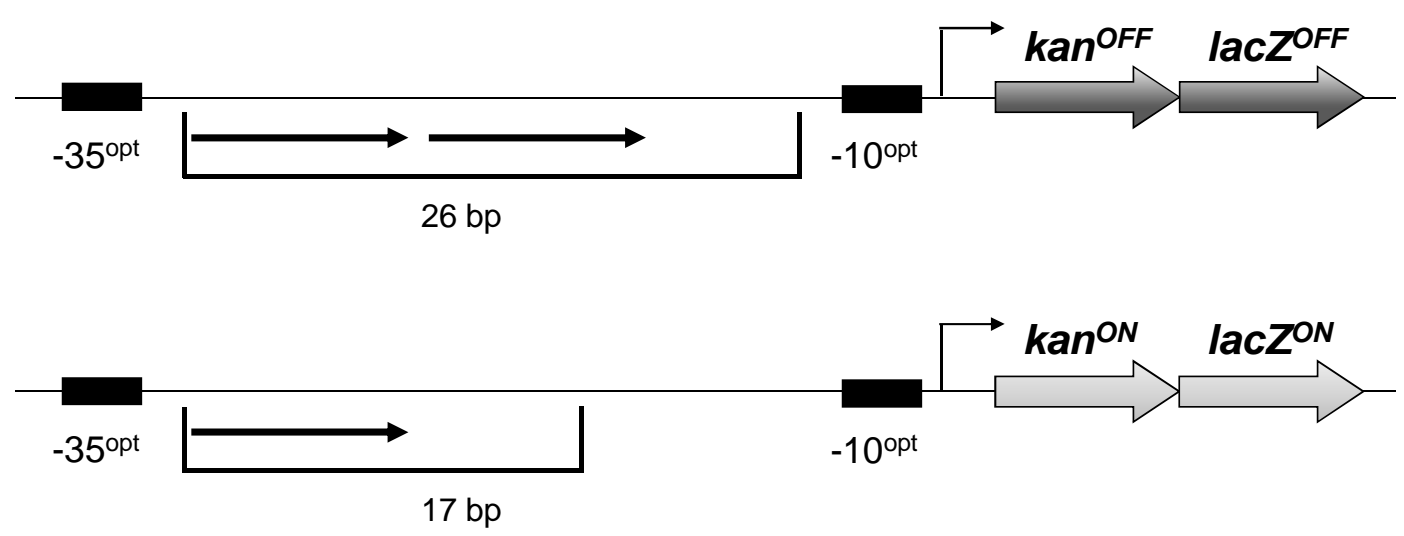

Fig. 4.2 - The mutagenesis test system.

The direct repeat originating from the $\operatorname{gudB}$ allele of $B$. subtilis was placed as the spacer between an optimal -10 and -35 region (upper part). An operon consisting of a kanamycin resistance gene (aphA3) and the $\beta$-galactosidase gene (lacZ) was placed under the control of the artificial promoter. Due to the long spacer, the promoter is not active. By the precise deletion of nine bp in the spacer region the promoter gains function and the kanamycin resistance und the $\beta$-galactosidase are highly expressed (lower part).

Such a reporter system was constructed as described in Material and Methods and introduced into the genome of B. subtilis, resulting in strain GP1123 (see Tab. S3). $B$ subtilis GP1123 was unable to grow in the presence of kanamycin and formed white 
colonies on plates containing X-Gal suggesting that neither kanamycin resistance nor $\beta$ galactosidase was expressed by these bacteria. These findings demonstrate that the alf promoter was inactive, as expected. However, we observed the sporadic appearance of kanamycin-resistant blue colonies that might result from the activation of the alf promoter. Indeed, a sequence analysis of the promoter for several colonies revealed the deletion of one part of the repeat resulting in a promoter (alfl) with perfect -10 and -35 regions separated by the preferred distance of $17 \mathrm{bp}$. Thus, the deletion of one part of the repeat occurs also in a non-related sequence context.

Next, we asked whether the deletion of the repeat in the alf promoter took place with a similar high frequency as observed for the decryptification of $g u d B$. For this purpose, the rate of appearance of kanamycin-resistant suppressor mutants of GP1123 was determined. It was found to be $1.3 \times 10^{-7}$. This mutation rate is in the range typically observed in bacteria (Kunkel, 2004), but three orders of magnitude lower than the rate found for the deletion event in the $\operatorname{gudB}$ gene context. Thus, there seems to be a relevant difference between the sequence contexts of the $\operatorname{gudB}$ gene and the alf promoter that results in drastically changed mutation rates.

\section{Expression of the gudB gene and stability of the cryptic and active glutamate dehydrogenases}

While the direct repeat is part of a putatively expressed coding region in the $\operatorname{gudB}$ gene, it is present in the non-transcribed spacer in the artificial alf promoter. This difference might contribute to the different mutation rates observed in the two sequence contexts. Therefore, we decided to study first the expression of the gudB gene to some detail. Previous studies have shown that $g u d B$ expression is not modulated by the source of nitrogen present in the medium (Belitsky \& Sonenshein, 1998). We have studied the activity of the $g u d B$ promoter by determining the expression of a $g u d B$-lacZ fusion in wild type, $\Delta \operatorname{roc} G$ and $g u d B 1$ genetic backgrounds. As shown in Tab. 4.1, the fusion was expressed constitutively irrespective of the genetic background or medium analyzed. The expression level of about 500 units/mg of protein is quite high as compared to other lacZ fusions based on the same reporter system (Schilling et al., 2007). Thus, even the cryptic gudB gene coding for an inactive protein is constantly expressed at high levels in B. subtilis. 
To allow the action of selective pressure on the decryptification of $\operatorname{gudB}$, the accumulation of the active protein is required. However, the inactive GudB protein was reported to be one of the most unstable proteins of B. subtilis (Gerth et al., 2008). In contrast, preliminary evidence suggested that the active GudB1 protein is much more stable (Gunka et al., 2010). The issue of stability might apply not only at the level of the protein, but may also be relevant for the $g u d B$ mRNA. To address these problems, we first determined the stability of the gudB mRNA of B. subtilis GP747 and the isogenic gudB1 mutant GP753 by a Northern blot analysis. As shown in Fig. 4.3A, we detected a single transcript of about $1,300 \mathrm{bp}$ for $g u d B$. This corresponds to a monocistronic transcript and is in good agreement with previous suggestions based on genome analysis (Belitsky \& Sonenshein, 1998). The quantitative evaluation of the mRNA stability revealed a half-life of about $4 \mathrm{~min}$. The stability of the mRNA was similar in both strains, demonstrating that it is not affected by the presence of the direct repeat.

Tab.4.1 - Analysis of gudB expression.

$\beta$-Galactosidase activity ${ }^{\mathrm{a}}$

\begin{tabular}{ccccccc} 
Strain & Relevant genotype & C-Glc & CE & CE-Glc & CR & CR-Glc \\
\hline GP1101 & wild type & 357 & NG & 504 & 573 & 415 \\
GP1102 & gudB1 & 182 & 384 & 268 & 415 & 242 \\
GP1104 & rocG::Tn10 & 422 & NG & 557 & NG & 394 \\
GP1105 & rocG::Tn10 gudB1 & 225 & 658 & 478 & 410 & 295
\end{tabular}

a Bacteria were grown in $\mathrm{C}$ minimal medium. Glucose (Glc), glutamate (E), and arginine (R) were added to final concentrations of $5 \mathrm{~g} / \mathrm{l}(\mathrm{Glc}$ and $\mathrm{R})$ or $8 \mathrm{~g} / \mathrm{l}(\mathrm{E})$. $\beta$-Galactosidase activities are given as units/mg of protein. Experiments were carried out at least threefold. Representative results from one series are shown.

$\mathrm{NG}=$ no growth

The accumulation of the glutamate dehydrogenase GudB was studied by Western blot analysis. For this purpose, we used the cell extracts of the rocG mutant GP747 and its isogenic $g u d B 1$ derivative GP753 that were prepared for the 
determination of the mRNA stability (just prior to rifampicin addition, $\mathrm{t}_{0}$ in Fig. 4.3A). To detect the GudB protein, we used an antibody raised against RocG. Both proteins are very similar and the antibody recognizes GudB as well. Since both strains used for this experiment are $\operatorname{roc} G$ mutants, the only signal is obtained with GudB (Commichau et al., 2007b).

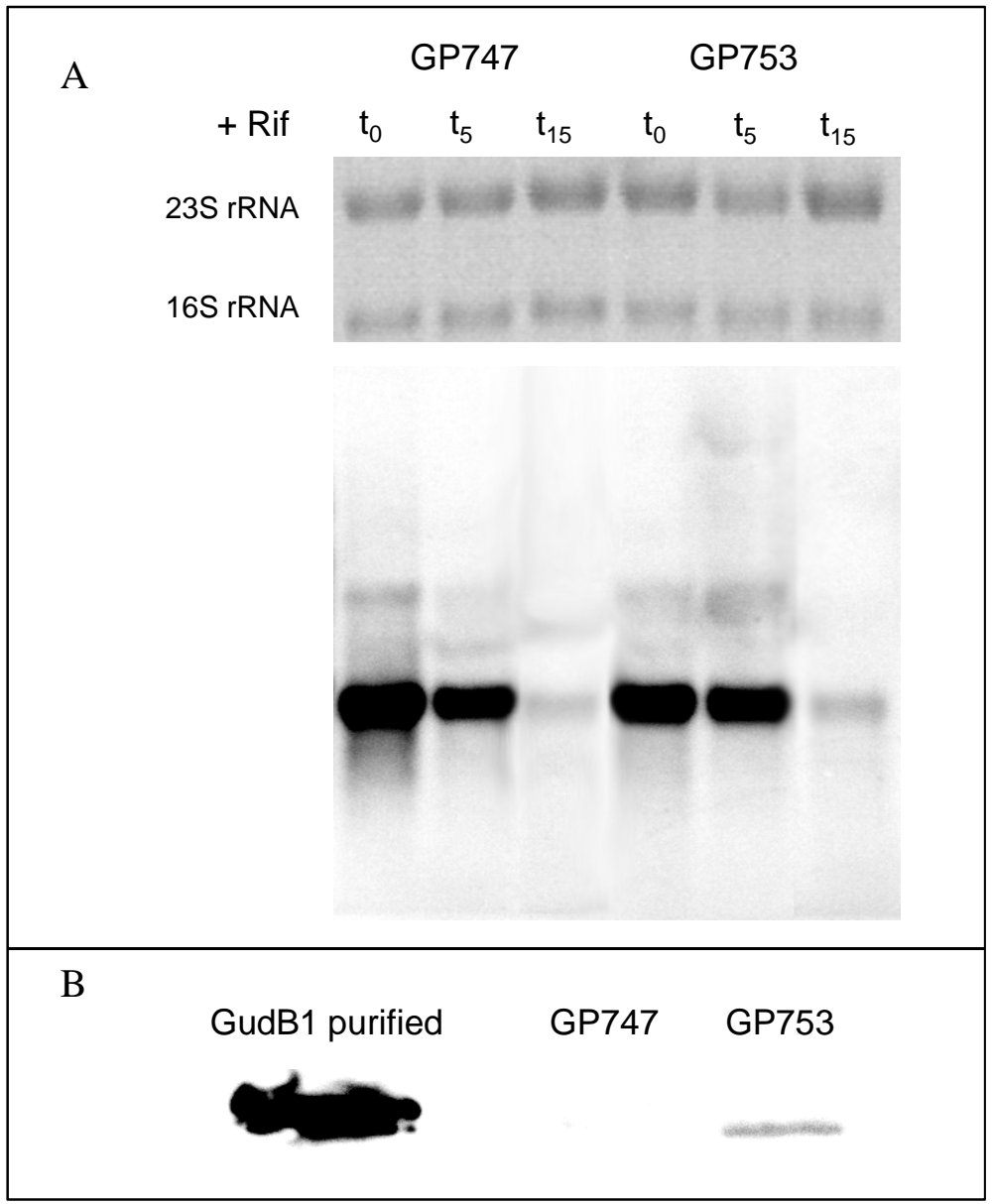

Fig. 4.3 - Expression of the gudB gene and the GudB protein level.

(A) Northern blot analysis was performed to determine the stability of the gudB mRNA of B. subtilis GP747 and the isogenic gudB1 mutant GP753. Both mRNAs do not differ in stability and half-life, implying that the direct repeat does not influence mRNA levels. (B) A Western blot analysis was performed to compare the protein levels of GudB with the level of GudB1. Crude extracts of B. subtilis GP747 and the isogenic gudB1 mutant GP753 were used and the GudB protein was detected by using antibodies raised against RocG that cross-react with the GudB protein. 
As shown in Fig. 4.3B, the active enzyme GudB1 could be detected in the extract of GP753. In contrast, no signal was observed for the cryptic GudB protein. Since the mRNA amounts are similar for both strains (see Fig. 4.3A), we may conclude that the inactive GudB protein is highly unstable, as suggested by a previous study (Gerth et al., 2008). In contrast, the active glutamate dehydrogenase GudB1 is a stable protein that accumulates in the cell. Thus, the decryptification of $\operatorname{gudB}$ is sufficient for the cell to obtain immediately an active glutamate dehydrogenase that may help to overcome the metabolic imbalance of the $\operatorname{roc} G$ mutant.

\section{Implication of repair and recombination proteins in the decryptification of $g u d B$}

The extremely high frequency at which the $\operatorname{gudB}$ decryptification occurs and the fact that the rate is much higher in the $\operatorname{gudB}$ locus as compared to the alf mutagenesis reporter system suggest the involvement of proteins in the mutagenesis process. The sequence of the direct repeat in $\operatorname{gudB}$ is somewhat similar to the chi sequence that is recognized and bound by the $\mathrm{Add} A \mathrm{~B}$ helicase/nuclease, a component of the recombination machinery of the cell. Since the recombination protein RecA is also involved in the generation of mutations, we determined the gudB mutation rate of the $a d d A B$ and recA mutant strains GP1107 and GP1103, respectively. The rates were similar to those observed with the isogenic roc $G$ mutants $\left(0.9 \times 10^{-4}\right.$ and $1.1 \times 10^{-4}$ for the $a d d A B$ mutant and the wild type GP754; $0.3 \times 10^{-4}$ vs. $1.3 \times 10^{-4}$ for the rec $A$ mutant and the wild type GP747). Therefore, AddAB and RecA do not seem to play a major role in the deletion of the direct repeat in the $g u d B$ gene.

If $\mathrm{AddAB}$ and RecA would have played a role in the deletion of the direct repeat in $\operatorname{gudB}$, we would have expected that they are not selective for the $g u d B$ gene context as compared to the context of the alf promoter. Thus, the genetic context plays a decisive role in the decryptification of $g u d B$. As shown above, the $g u d B$ gene is constitutively expressed. In contrast, the core promoter of the mutagenesis reporter system is a non-transcribed region. The transcription-repair coupling factor Mfd might therefore participate in the deletion of the $\operatorname{gudB}$ repeat. To test this idea, we constructed the $m f d$ deletion mutant GP1169 and compared the mutation rate in this strain to that of the isogenic $\operatorname{roc} G$ mutant GP747. In this case, we detected a hundred-fold reduction of the frequency of gudB1 mutants $\left(1.25 \times 10^{-6}\right.$ vs. $\left.1.3 \times 10^{-4}\right)$. Next, we investigated the impact of the $m f d$ mutation on the deletion of the repeat in the alf promoter. In this case, 
the mutation rates of the wild type (GP1123) and the isogenic mfd mutant GP1168 were very similar $\left(1.3 \times 10^{-7}\right.$ vs. $\left.2.3 \times 10^{-7}\right)$. Thus, the $m f d$ mutation affects the deletion of the direct repeat only in the context of the transcribed gene. This observation strongly supports the idea that transcription of the $g u d B$ gene is essential for obtaining the high rate of decryptification.

\section{Discussion}

In bacteria, mutations occur with a frequency of about $10^{-7}$ and the frequency of beneficial mutations is estimated to be even two orders of magnitudes less frequent (Imhof \& Schlötterer, 2001; Kunkel, 2004). The gudB1 mutation studied in this work appeared with a rate of about $10^{-4}$. To the best of our knowledge, this is the highest mutation rate for a specific allele that has ever been observed in bacteria.

Since the pioneering work of Darwin it was thought that the occurrence of a mutation is a stochastic event that may affect each nucleotide with the same probability and that natural selection provides the choice between different variants (Darwin, 1859). However, research in the last few years suggests that selective pressure may somehow favor the appearance of beneficial mutations (Cairns et al., 1988; Roth et al., 2006). A recent long-term study with $E$. coli suggested that early during starvation the cells acquire the most beneficial mutations (i.e. those mutations that have the highest positive impact on fitness) and that independent bacterial cultures are likely to accumulate the same beneficial mutations (Barrick et al., 2009). The high frequency and the high precision of the $\operatorname{gudB}$ decryptification have two implications: First, a specific selective pressure seems to stimulate the occurrence of the $g u d B 1$ mutation. Second, the molecular tools to generate the mutation must be present in B. subtilis.

Previous work has shown that glutamate homoeostasis is important for both E. coli and B. subtilis (Yan, 2007; Commichau et al., 2008). To any mutation that affects glutamate metabolism, the bacteria respond readily with the emergence of suppressor mutations. In the lab strain of B. subtilis, the inactivation of $g u d B$ seems to be very stable under laboratory growth conditions. Indeed, a derivative of the strain 168 with an active GudB glutamate dehydrogenase could only be selected on minimal medium with glutamate as the single carbon source (Belitsky \& Sonenshein, 1998; Commichau et al., 2008). The situation is completely different when the rocG gene that 
encodes the final enzyme of the arginine degradation pathway is inactivated. These bacteria form only small translucent colonies on complex medium and rapidly acquire the $g u d B 1$ mutation. The occurrence of the $g u d B 1$ mutation at such a high frequency suggests the existence of a strong selective pressure exerted on the rocG mutant. The roc $G$ gene product, the glutamate dehydrogenase, converts glutamate to 2-oxoglutarate. This suggests that glutamate or one of its precursors in the arginine degradation pathway might accumulate in the $\operatorname{roc} G$ mutant and this might be problematic for the cell. We have tested the growth of mutants affected in the different steps of arginine degradation on complex medium; however, the strong growth defect was unique to the rocG mutant (our unpublished results). Thus, the accumulation of glutamate may be toxic for the cell. This idea is in good agreement with the observation that a strain with a constitutive high-level expression of the glutamate synthesizing enzyme glutamate synthase acquired a mutation that inactivates this enzyme when grown in the presence of glutamate (Commichau et al., 2008). This leaves us with the question why glutamate should be toxic for the cell when it is the most abundant metabolite anyway. The enzyme glutamate racemase (encoded by the essential gene racE in B. subtilis) catalyzes the conversion of L-glutamate to D-glutamate that is a building block for peptidoglycan biosynthesis (Kimura et al., 2004; Spies et al., 2009). Indeed, the accumulation D-glutamate was shown to be toxic for B. subtilis (Kimura et al., 2004). In the presence of very high intracellular amounts of L-glutamate due to the strong induction of the enzymes of the arginine degradation pathway, RacE probably generates higher concentrations of D-glutamate than tolerated by the cell. The activation of the normally cryptic glutamate dehydrogenase GudB might then bring the glutamate concentration to a level that does not longer result in the accumulation of harmful Dglutamate.

A mutation can only be beneficial for a bacterium if it confers the cell with an immediate selective advantage. This was observed in the case of long-term evolution of E. coli: most of the mutations that were fixed after 5,000 generations resulted in an increased fitness of the cells (Barrrick et al., 2009). In the case of GudB, a mutation has two solve two problems at the same time: first, the extremely unstable protein needs to be stabilized and, second, the stable enzyme must bring an advantage for the cell. As shown in this work, the decryptification of GudB is accompanied by drastic increase of the stability of the protein as judged from the increased amounts of the protein while the 
expression of the gene is not affected by the mutation. The active GudB protein may than participate in reducing the cellular glutamate pool as outlined above. Thus, the decryptification of $g u d B$ meets both requirements.

A particular feature of the $g u d B$ gene is the presence of a perfect direct repeat. To our knowledge, this is a unique situation in the genome of B. subtilis. As shown in this work, the repeat is essential for the high rate of $g u d B$ decryptification. Tandem repeats in bacteria are usually instable and multiple mechanisms are involved in their contraction or expansion (Bichara et al., 2006). However, the well-studied tandem repeats such as the $\operatorname{lgt} C$ repeat in Haemophilus influenzae or the nadA repeat in Neisseria meningitidis consist of five to 36 repeats of tetranucleotides (Bayliss et al., 2001; Martin et al., 2005). In those cases, the instability is not linked to transcription (Bichara et al., 2006). The $g u d B$ repeat is unique in possessing a large repeat (unit repeat of nine nucleotides) that is present in only two copies. In contrast to the wellstudied tandem repeats the $g u d B$ repeat is very stable in a non-related, non-transcribed genomic context. Our work provides first insights into the mechanism of the deletion of one part of the $g u d B$ repeat: The Mfd protein is essential for the high-frequency decryptification of $\operatorname{gudB}$ if the repeat is located in a transcribed region, and then the first part of the repeat is preferentially deleted. The identification of the particular enzymes that are required for the decryptification of $g u d B$ will be the subject of further analyses.

The Mfd-mediated coupling of transcription to DNA repair and mutagenesis can be regarded as a built-in precaution that facilitates the accumulation of mutations preferentially in transcribed genes. This has several implications: (i) The coupling allows that the mutations occur in genes that are expressed at the given timepoint; therefore the mutant variants of the encoded proteins might help to overcome the actual limitation. (ii) Non-transcribed genes that may be required under different conditions are in this way protected from potentially harmful mutations. Both effects facilitate the adaptation of bacteria to all kind of challenges that limit their growth and are therefore crucial for bacterial evolution. 


\section{Acknowledgements}

Kristin Kaiser is acknowledged for the help with some experiments. We are grateful to Dusko Ehrlich and Alan Grossman for providing us with strains HVS666 and IRN444, respectively. We wish to thank Marie-Francoise Noirot-Gros, Hinnerk Eilers and Wilfried Kramer for helpful discussions. This work was supported by the DFG, the Federal Ministry of Education (Research SYSMO network (PtJ-BIO/0313978D) and the Fonds der Chemischen Industrie to J. S. 


\section{Chapter 5}

Further factors involved in the Mfd-dependent decryptification of the gudB allele in Bacillus subtilis 


\begin{abstract}
In the laboratory Bacillus subtilis strain 168 the $\operatorname{gudB}$ gene encoding an inactive glutamate dehydrogenase is rapidly decryptified upon deletion of the $\operatorname{roc} G$ gene coding for the active glutamate dehydrogenase. This decryptification occurs at a high mutation rate of about $10^{-4}$. In the decryptified $g u d B$ allele, designated $g u d B 1$, nine base pairs of a direct repeat in the coding region are precisely excised, leading to the production of an active enzyme. A recent study showed that the high rate of $g u d B 1$ mutation in a $\operatorname{roc} G$ mutant background depends on the perfect direct repeat and the presence of the Mfd protein. The involvement of Mfd, which is a transcription repair coupling factor, in the gudB1 mutations implies the contribution of other proteins that are part of the DNA repair system to the mutation event. In order to identify these putative factors, mutants that are deficient in the nucleotide excision repair and the DNA repair mismatch systems were constructed. Both, the loss of the UvrA and UvrB proteins and the MutS and MutL proteins do not reduce the $\operatorname{gudBl}$ mutation rate as much as the Mfd deficiency. These results support the idea that other factors may operate together with Mfd in the $g u d B 1$ decryptification.
\end{abstract}

\title{
Introduction
}

The genome of B. subtilis codes for two glutamate dehydrogenases, RocG and GudB. In the laboratory strain 168 only the RocG protein is active in degrading glutamate to 2-oxoglutarate and ammonium (Belitsky \& Sonenshein, 1998), whereas the $\operatorname{gudB}$ gene encodes a cryptic glutamate dehydrogenase that cannot utilize glutamate as a source carbon (Belitsky \& Sonenshein, 1998; Zeigler et al., 2008). The inactivity of the GudB protein is caused by a duplication of nine base pairs in the coding region of the $\operatorname{gudB}$ gene leading to the duplication of the three amino acids in the active site of the protein (Belitsky \& Sonenshein, 1998; Commichau et al, 2007b; Gunka et al., subm.).

In the absence of the active glutamate dehydrogenase RocG, B. subtilis is not capable of utilizing glutamate anymore which results in a severe growth defect of a rocG mutant on medium containing glutamate and a variety of its precursors. This growth defect is rapidly suppressed by a mutation which activates the cryptic $\operatorname{gudB}$ allele. It was demonstrated that the suppressor mutation always occurs as the precise 
deletion of nine base pairs of the direct repeat in the $g u d B$ gene (Belitsky \& Sonenshein, 1998, Commichau et al., 2007a). The decryptification of the gudB allele occurs at an extremely high rate $\left(1.3 \times 10^{-4}\right)$ (Gunka et al., subm.). By this mutation the gudB allele, designated as $\operatorname{gudB1}$, gains function and codes for a stable and active glutamate dehydrogenase (Belitsky \& Sonenshein, 1998).

As shown, there are at least two requirements for the rapid decryptification of the gudB allele. (i) The presence of a perfect direct repeat and (ii) and the transcription repair coupling factors Mfd are crucial for the high mutation rate (Gunka et al., subm.). The lack of one of these requirements reduces the mutation rate about hundred-fold, respectively.

The Mfd protein is evolutionarily highly conserved and belongs to the group of transcription repair coupling factors (TCRF). These factors recognize stalled RNA polymerase caused by a DNA lesion. TRCFs displace the RNA polymerase and its transcripts and recruit the DNA excision repair machinery that resolves the lesion and allows the restart of transcription (Roberts \& Park, 2004; Borukhov et al., 2005; Deaconescu et al., 2006). Until now, little is known about the molecular mechanism of the detailed recruitment of the DNA excision repair machinery by the TCRF Mfd. But evidence was provided that Mfd directly interacts with the UvrA subunit of the UvrABC nucleotide excision repair system (Selby \& Sancar, 1993; Selby \& Sancar 1995a).

Furthermore, it was shown that the Mfd protein is involved in the phenomenon of stationary phase mutagenesis in B. subtilis (Ross et al., 2006; Robleto et al., 2007; Pybus et al., 2010). Indeed, in a B. subtilis strain lacking the Mfd protein the accumulation of beneficial mutations is diminished. This is in good agreement with our findings that the frequency of $g u d B 1$ mutations is decreased in a $m f d$ mutant strain (Gunka et al., subm.).

Recently, it was demonstrated that another role of the Mfd protein is to clear the conflict between the DNA and the RNA polymerase in a head-on collision in E. coli. The Mfd protein arranges to solve this conflict in favor of replication by pausing transcription (Pomerantz \& O’Donnell, 2010).

To further elucidate the molecular mechanism of the Mfd-dependent rapid $\operatorname{gudB}$ decryptification, it was studied which factors also contribute to the generation of $g u d B$ suppressors. Therefore, mutants of components of the UvrABC DNA excision repair 
system and the MutSL proteins that participate in the DNA repair mismatch were generated. Experimental evidence cannot be provided that any of the proteins analyzed is involved in the case of the $\operatorname{gudBl}$ mutation. Thus, it is still an open question with which DNA repair machinery Mfd operates in the $g u d B$ decryptification.

\section{Materials and Methods}

\section{Bacterial strains and growth conditions}

All B. subtilis strains used in this work are derived from the laboratory wild type strain 168. They are listed in Tab. S3. B. subtilis was grown in SP medium, LB medium or in $\mathrm{C}$ minimal medium supplemented with tryptophan (at $50 \mathrm{mg} / \mathrm{l}$ ) (Wacker et al., 2003). CSE medium is C minimal medium supplemented with sodium succinate $(6 \mathrm{~g} / \mathrm{l})$ and potassium glutamate $(8 \mathrm{~g} / \mathrm{l})$. $\mathrm{C}$-Glc is $\mathrm{C}$ minimal medium supplemented with glucose $(5 \mathrm{~g} / \mathrm{l})$, and CS is supplemented with sodium succinate $(6 \mathrm{~g} / \mathrm{l})$ (Wacker et al., 2003). LB, SP and CS plates were prepared by the addition of $17 \mathrm{~g}$ Bacto agar/l (Difco, Lawrence, USA) to LB, SP or CS medium, respectively.

\section{Construction of mutant strains}

The $\triangle m u t S L$ and $\triangle u v r A B$ mutant strains were obtained by applying the long flanking homology PCR (LFH-PCR) technique (Wach et al., 1996). For the construction of the $u v r A B$ mutant, erm $C$ resistance gene was amplified from pDG647 with the primers mls-fwd (kan)/mls-rev (kan) (Guérot-Fleury et al., 1995). Internal DNA fragments of about 1,000 bp flanking the $u v r A B$ region at its 5' and 3' end were amplified using the primer pairs KG114/KG115 and KG116/KG117. All primer sequences are provided in Tab. S1. Chromosomal DNA of B. subtilis 168 served as the template. The 3' end of the upstream fragment as well as the 5' end of the downstream fragments extended into the $u v r A B$ gene region, in a way that all expression signals of genes up- and downstream remained intact. The joining of the two fragments to the resistance cassette was performed in a second PCR. In this reaction we used the primer pair KG114/KG117 for the deletion of the $u v r A B$ region. The PCR product was directly used to transform B. subtilis 168 resulting in the mutant GP1175. 
The $\Delta m u t S l$ mutant GP1190 was obtained in a similar way. Briefly, the aphA3 resistance gene was amplified from pDG780 with the primers mls-fwd (kan)/mls-rev (kan) (Guérot-Fleury et al., 1995), and the regions flanking the mutSL genes were amplified using the primer pairs KG127/KG128 and KG129/KG130. The joining PCR was performed with the primer pairs KG127/KG130. The PCR product was used to transform B. subtilis 168. The integrity of the regions flanking the integrated resistance cassette was verified by sequencing. PCR products of about 1,000 bp were amplified from chromosomal DNA of the resulting strain GP1190 by using the primer pairs KG131/kan-check rev and KG132/kan-check fwd.

\section{Determination of mutation rates}

The gudB mutation rates of the B. subtilis strains GP1176, GP1191 and GP1192 were determined as previously described in Gunka et al., subm.

\section{DNA manipulation}

B. subtilis was transformed with chromosomal DNA or PCR products according to the two-step protocol described previously (Kunst \& Rapoport, 1995). Transformants were selected on SP plates containing kanamycin (Kan $10 \mu \mathrm{g} / \mathrm{ml}$ ), spectinomycin (Spec $150 \mu \mathrm{g} / \mathrm{ml}$ ) or erythromycin plus lincomycin (Erm $2 \mu \mathrm{g} / \mathrm{ml}$ and Lin $25 \mu \mathrm{g} / \mathrm{ml}$ ).

Chromosomal DNA of B. subtilis was isolated as described (Kunst \& Rapoport, 1995). DNA sequences were determined using the dideoxy chain termination method (Sambrook et al., 1989). 


\section{Results}

\section{Involvement of the UvrAB proteins in the gudB mutation}

We observed a hundred-fold decrease in the $g u d B$ decryptification in the $m f d$ mutant strain GP1169 (Gunka et al., subm.). This suggests that the nucleotide excision repair NER system might also be involved in the precise excision of the nine base pairs in the $g u d B$ allele. The Mfd protein does not possess nuclease activity but is required for recruiting the NER machinery to a certain DNA lesion in the process of the transcription coupled nucleotide excision repair (TCR) (Selby \& Sancar, 1993; 1994; Deaconescu et al., 2006).

As a potential candidate for the excision system, operating with the Mfd protein in the $\operatorname{gudB}$ decryptification, the UvrABC system was chosen. In E. coli, the UvrA protein is able to interact with transcription repair coupling factors (TCRFs) like Mfd leading to the repair of the damaged DNA (Truglio et al., 2006). Thus, the $u v r A B$ operon was deleted in a $\operatorname{roc} G$ mutant strain (GP1176) and the $g u d B$ mutation rate was determined (Gunka et al., subm.). The mutation rate of the $u v r A B$ mutant strain was similar to those observed with the isogenic $\operatorname{roc} G$ mutant GP747 (0.1 x $10^{-4}$ and $1.3 \times 10^{-4}$, respectively).

If UvrABC would be involved in the excision of the nine base pairs in the $\operatorname{gudB}$ allele, we would expect a more striking effect of an $u v r A B$ deletion on the mutation rate. In conclusion, the UvrABC system does not seem to operate with Mfd in the generation of $g u d B$ suppressors.

\section{Effect of a mutSL gene deletion on the gudB mutation}

In order to gain further insights into the molecular mechanism of the gudB decryptification, we looked for other interaction partners of the Mfd protein that could have the capacity to repair DNA damage. For E. coli it was shown, that the deletion of the DNA repair mismatch genes mutS and mutL selectively abolishes DNA repair in the transcribed DNA strand to the same extent as a $m f d$ deletion does (Mellon et al., 1996). Therefore, both the mutS and the mutL gene were deleted and it was tested whether these deletions have an impact on the mutation rate of the $g u d B$ allele. We determined the mutation rate of the $\operatorname{roc} G$ mutSL double mutant (GP1191) in comparison to its isogenic $\operatorname{roc} G$ parent GP747. The mutation rate was $0.2 \times 10^{-4}$ for the mutSL mutant strain GP1191 which is similar to the rate of the $\operatorname{roc} G$ mutant GP747 (1.3 x 10 $\left.0^{-4}\right)$. Thus, 
the deletion of mutS and mutL has no significant effect on the precise excision of the nine base pairs in the $g u d B$ allele.

To exclude the possibility that the UvrAB and the MutSL proteins can replace each other in the transcription coupled nucleotide excision repair, we constructed a B. subtilis strain lacking the $u v r A B$ and the mutSL genes (GP1192). The mutation rate of the $\operatorname{gudB}$ gene in this strain was $0.13 \times 10^{-4}$ which is similar to its isogenic rocG mutant strain GP747 $\left(1.3 \times 10^{-4}\right)$. This result leads to two conclusions: First, it can be assumed that the UvrA and the UvrB proteins cannot be displaced by the proteins MutS and MutL and vice versa in the process of gudB decryptification. Secondly, we propose that neither the nucleotide excision repair proteins UvrA and UvrB nor the DNA repair mismatch protein MutS and MutL contribute to the Mfd mediated gudB mutation.

\section{Discussion}

The data presented herein, imply that in the case of the $\operatorname{gudBl}$ mutation the Mfd protein does not act in concert with the UvrABC or the MutSL systems. Even the simultaneous inactivation of both systems does not lead to a significant decrease in the $\operatorname{gudB1}$ mutation rate.

Mfd possess structural characteristics which are highly conserved (Deaconescu et al., 2006). Allowing six gaps, the amino acids 15 to 473 of the Mfd protein of B. subtilis share a $19 \%$ identity with the N-terminal region of UvrB protein of B. subtilis see (Fig. 5.1). This region in the UvrB protein is supposed to contain residues necessary for the interaction with the UvrA protein. Therefore, the N-terminal part of the Mfd protein seems to be necessary for the recruitment of the NER system by interacting with UvrA (Selby \& Sancar, 1993). As the very last C-terminal part of UvrB was shown to interact with the UvrC protein and this part is not homologous to any part of the Mfd protein, it is unlikely that Mfd itself can interact with UvrC. It was demonstrated that the Mfd protein interacts with the RNA polymerase (see Fig. 5.1) and is therefore sufficient to recognize a stalled transcription complex (Ayora et al., 1996). The C-terminal domain of Mfd is supposed to block the UvrA interaction site until a stalled RNA polymerase is recognized by the RNA polymerase interaction domain and displaced by the translocation domain (Deaconescu et al., 2006). 


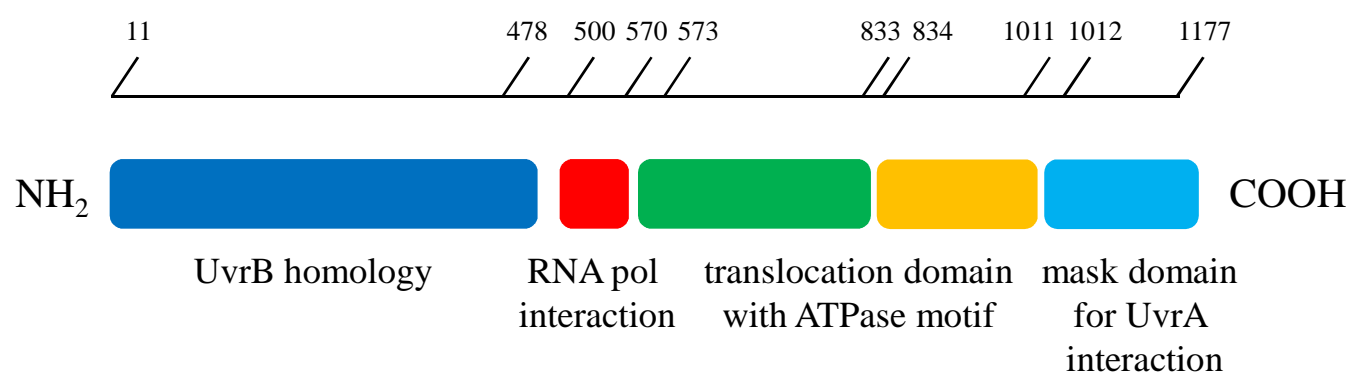

Fig. 5.1 - Domain structure of the B. subtilis Mfd protein.

Characteristic and conserved domains of TCRFs are mapped on the primary structure of the B. subtilis Mfd protein (Illustration adapted to Deaconescu et al., 2006). Each domain is highlighted in a different color. The domains were determined by using a multiple alignment of Mfd homologues to (Deaconescu $e t$ al., 2006) (RNA pol = RNA polymerase).

It is obvious that the Mfd protein can neither recruit UvrC nor can it perform nucleotide excision itself. Therefore, it is tempting to speculate that the Mfd mediated generation of the gudBl mutation occurs in yet unknown mechanism and involves proteins that have not been linked with the transcription coupled nucleotide excision repair until now.

\section{Future perspectives}

In order to gain insights into the Mfd-mediated generation of $\operatorname{gudB1}$ mutations a SPINE experiment using a roc $G$ mutant strain can be performed (Herzberg et al., 2007). By this in vivo cross linking approach potential interaction partners of the Mfd protein can be identified. To increase the chance of cross-linking Mfd in complex with its potential target, the $\operatorname{gudB}$ gene, and additional interaction partners, the $\operatorname{gudB}$ gene has to be enriched. Besides the analysis of the elution fraction by mass spectrometry to identify Mfd interaction partners, a Northern blot with a $\operatorname{gudB}$ probe can be performed. This experiment might demonstrate whether the $\operatorname{gudB}$ transcript can be specifically cross-linked with the Mfd protein. 


\section{Chapter 6}

SPABBATS: A pathway-discovery method based on Boolean satisfiability that facilitates the characterization of suppressor mutants

The work described in this chapter was submitted for publication in:

Flórez, L. A., Gunka, K., Polanía, R., Tholen, S. \& Stülke, J. SPABBATS: A pathway-discovery method based on Boolean satisfiability that facilitates the characterization of suppressor mutants. BMC Systems Biology submitted

Author contributions:

LAF, KG, and JS planned the project. LAF and RP conceived and implemented the SPABBATS algorithm. KG and JS developed the strategies for the experimental validation of the method. ST contributed to the performance of the experiments. LAF, KG, and JS wrote the manuscript and created the Table and Figures. All authors read and approved the final manuscript. 


\begin{abstract}
Background

Several computational methods exist to suggest rational genetic interventions that improve the productivity of industrial strains. Nonetheless, these methods are less effective to predict possible genetic responses of the strain after the intervention. This problem requires a better understanding of potential alternative metabolic and regulatory pathways able to counteract the targeted intervention.
\end{abstract}

\title{
Results
}

Here we present SPABBATS, an algorithm based on Boolean satisfiability (SAT) that computes alternative metabolic pathways between input and output species in a reconstructed network. The pathways can be constructed iteratively in order of increasing complexity. SPABBATS allows the accumulation of intermediates in the pathways, which permits discovering pathways missed by most traditional pathway analysis methods. In addition, we provide a proof of concept experiment for the validity of the algorithm. We deleted the genes for the glutamate dehydrogenases of the Grampositive bacterium Bacillus subtilis and isolated suppressor mutant strains able to grow on glutamate as single carbon source. Our SAT approach proposed candidate alternative pathways which were decisive to pinpoint the exact mutation of the suppressor strain.

\section{Conclusion}

SPABBATS is the first application of SAT techniques to metabolic problems. It is particularly useful for the characterization of metabolic suppressor mutants and can be used in a synthetic biology setting to design new pathways with specific input-output requirements. 


\section{Background}

A holistic understanding of cellular metabolism is central to systems biology and metabolic engineering: In order to amplify the flux through production pathways in industrial strains we have to understand how the metabolic network responds to our interventions.

Several methods can suggest rational interventions that may lead to favorable industrial phenotypes (Feist \& Palsson, 2008). Their goal is to optimize the distribution of metabolic fluxes towards the product of interest, either directly (e.g. FBA, MOMA or ROOM) or indirectly by coupling it to another characteristic (e.g. OptKnock) that facilitates further strain improvements via mutation and screening.

While these methods can predict a final flux distribution, they do not predict the range of genetic and metabolic responses of the organism after the targeted mutation. At the same time, it would be highly desirable to have tools that may predict these responses, since they can suggest ways to generate more stable strains, or accelerate the adaptation to an intended optimal flux. The challenge of the question is the need to understand why particular genetic responses make sense in an evolutionary setting. Thus, the ultimate question is: Which parallel pathways - that were not active previously - result in an adaptive advantage under the screening conditions?

Pathway analysis has received increased attention due to the reconstruction of genome scale metabolic networks for many organisms. These methods can be divided into two categories: stoichiometric and path oriented (Planes \& Beasley, 2008). The first approach generates all pathways that conform to the pseudo-steady-state assumption for internal metabolites. However, it presents two problems: the number of predicted pathways is in the order of millions for genome scale models, making the approach totally intractable for the question at hand (Klamt \& Stelling, 2002). Its second shortcoming is the constraint imposed by the pseudo-steady-state assumption for internal metabolites. This assumption may rule out feasible pathways or (in case we include a large number of "freely available" metabolites) result again in a combinatorial explosion of pathways. The alternative approach - path oriented pathway reconstructions - is advantageous since it usually generates a small (and thus tractable) set of possible pathways. This is due to the choice of starting and ending metabolites and heuristics on the characteristics of the "optimal" pathway. However, the path- 
oriented approach may result in unrealistic pathways that consume internal metabolites not present in sufficient quantities inside the cell.

What is needed is an algorithm that reconstructs stoichiometrically balanced pathways in increasing order of complexity, with relaxed mass-balance constraints in comparison to the traditional pseudo-steady-state restriction.

A solution based on mixed-integer linear programming (MILP) has been suggested by de Figueiredo et al., but it has not been used in an evolutionary context so far (de Figueiredo et al., 2009). Here we describe the use of Boolean satisfiability (SAT) for the reconstruction of alternative pathways in metabolic networks (Claessen $e t$ al., 2009). Given a set of basal metabolites (that are considered freely available) and a set of target metabolites (whose concentration must increase), our SAT method constructs the shortest pathway between the basal and target sets (SPABBATS) of metabolites that is stoichiometrically balanced, while allowing the concentration of the intermediate metabolites to increase, if needed. The constraints are more relaxed than the ones for e.g. flux balance analysis, thus retaining the metabolically significant pathways. Using the algorithm iteratively, we obtain a prioritized list of pathways, whose elements can be tested individually by common molecular biology techniques.

To demonstrate the power of this concept, we applied the SPABBATS algorithm to a complex physiological problem, which is a result of an evolutionary experiment. We have elucidated a novel pathway of glutamate degradation present in the metabolic network of B. subtilis that had been decryptified upon inactivation of the normal glutamate catabolic genes. By using our SAT approach, we proposed four different new pathways that could be present in the mutant to utilize glutamate as single carbon source. These predictions were experimentally tested and revealed that one of these pathways was indeed active in the mutant strain and that this novel "suppressor" pathway is required and sufficient for glutamate utilization. This proves that the results of our approach correspond to valid metabolic alternatives for living cells. 


\section{Materials and Methods}

\section{Algorithm for finding short pathways between a basis and a target set of metabolites (SPABBATS)}

Our approach draws inspiration from flux-balance analysis (FBA) (Orth et al., 2010) in the sense that it searches the flux space of a metabolic network for fluxes that comply with a set of stoichiometric constraints. The major difference to FBA lies in the optimality criterion; in FBA the value to optimize is the target flux. In our case we change from optimization to satisfiability: we search for a flux that satisfies all the constraints, including a maximum number of allowed reactions.

Another important difference, that is a consequence of satisfiability approach, is that we use two variables for each flux instead of one. The first variable is a positive integer, which is a relative measure of the contribution of that particular flux to the total pathway. The second variable is Boolean and defines whether or not the particular flux takes part in the solution.

As in FBA, we define $\mathbf{S}$ as the stoichiometric matrix of the network with $n$ reactions and $m$ compounds. Reversible reactions are split into two unidirectional reactions. We divide the set of compounds into three disjoint sets:

i) $\quad \mathbf{B}$ is the set of basis compounds that are considered freely available, either because they are provided in the medium, or because they are "currency metabolites", whose concentration is buffered by the whole system (e.g. ATP, ADP, NADH, etc.)

ii) $\quad \mathbf{T}$ is the set of target compounds, the ones constrained to be produced in the pathways of interest

iii) I is the set containing all other compounds, that can be intermediates of the resulting pathway

We use different constraints for each of these sets. The compounds in the set $\mathbf{B}$ are left unconstrained. For each compound in the set $\mathbf{T}$, we write a constraint in the form:

$$
\sum_{i=1}^{n} s_{i j} a_{i} b_{i}>0
$$

where $s_{\mathrm{ij}}$ is the stoichiometric coefficient of compound $j$ in reaction $i$, and $a_{\mathrm{i}}$ and $b_{\mathrm{i}}$ are the integer and Boolean valued variables of reaction $i$, respectively. These 
constraints mean that in the solution pathway the overall flux to these metabolites should be positive.

For the compounds in the set I we use a constraint similar to (1), with the difference that we use a "greater than or equal to" $(\geq)$ sign. In FBA, an equality sign is used here, to constraint the fluxes to the steady-state space. We purposely do not constrain the pathway to the steady-state space, since the candidate solutions to the problem will not be the only pathway active in the cell and the intermediates that are accumulated in our pathway can be used by other pathways operating in parallel in the system. We require the total flux to these compounds to be non-negative, since the supposition is that they are not present in sufficiently high amounts to allow sustained growth on their consumption.

Next, we add constraints that limit the directionality of reversible reactions. This is done with constraints in the form:

$$
b_{i}+b_{j}<2 \text {, }
$$

where $b_{\mathrm{i}}$ and $b_{\mathrm{j}}$ are the Boolean variables of two reactions that together characterize a reversible reaction. These constraints mean that no two directions of a reversible reaction can appear in the final pathway at the same time.

Last, we add a constraint for the total length of the solution. This constraint is:

$$
\sum_{i=1}^{n} b_{i} \leq k
$$

where $k$ is a positive integer value that determines the maximum number of reactions that can appear in the pathway. This constraint does not immediately find the best solution, but it puts successively stricter upper-bounds to the maximum number of reactions that are allowed. Thus, it is able to find the shortest solution after some iterations by choosing successively smaller numbers for $k$.

The constraints for the compounds in $\mathbf{T}$ and $\mathbf{I}$ are not linear, since each term in the sum is composed of two variables instead of one. For this reason, a linear optimization strategy cannot be used directly. This limitation is not present when we use the SATsolver HySAT (Fränzle et al., 2007). It is able to find assignments to the variables that satisfy all the constraints in the system, even when these are non-linear. It is also able to detect if no such assignment exists. 
If the shortest solution has been found, the best sub-optimal solution can be found by adding an additional constraint in the form:

$$
\sum_{i \in K} b_{i}<k_{o p}
$$

where $k_{\mathrm{op}}$ is the number of reactions in the shortest solution and $K$ is the set of indices for the reactions in the shortest solution. In other words, we constrain the sum of all the Boolean variables of the optimal solution to be less than $k_{\mathrm{op}}$, thus leaving out the shortest solution from the solution space. By iterating this process with the Boolean variables of the sub-optimal pathway, we can find solutions with successively higher number of reactions.

The particular implementation of this algorithm for the problem mentioned in the Results section is as follows: we used the genome-scale reconstruction of B. subtilis (Oh et al., 2007). We removed the biomass "reaction"; it is useful for FBA, since it describes the target flux to cellular growth, but is meaningless in our context. In addition, we removed the reaction "glutamate dehydrogenase" (R_GLUDxi) to simulate the conditions of the strain GP717. We also scaled the non-integer stoichiometric coefficients of the model to integer values (and divided by the greatest common denominator). In our case, the set $\mathbf{B}$ contained the metabolites ATP, ADP, NAD ${ }^{+}$, $\mathrm{NADH}, \mathrm{FAD}, \mathrm{FADH}_{2}, \mathrm{H}_{2} \mathrm{O}, \mathrm{H}^{+}, \mathrm{NH}_{4}{ }^{+}$, and glutamate. These "currency metabolites" were chosen due to their participation in most catabolic pathways in the cell. The set $\mathbf{T}$ contained just 2-oxoglutarate. The remaining compounds were assigned to the set $\mathbf{I}$. We set the interval for the $a_{i}$ to $[1,1000]$. The calculations were done using an Intel Core2 Duo processor at $2.66 \mathrm{GHz}$, with $3.25 \mathrm{~GB}$ of RAM. The first pathway (the one involving leucine as intermediate) was found after 28 seconds. All other pathways took less than 8 minutes each to calculate.

\section{Bacterial strains and growth conditions}

All B. subtilis strains used in this work are derived from the laboratory wild type strain 168. They are listed in Tab. S3. E. coli DH5 $\alpha$ (Sambrook et al., 1989) was used for cloning experiments. B. subtilis was grown in $\mathrm{C}$ minimal medium containing ammonium as basic source of nitrogen (Wacker et al., 2003). Glutamate and/ or glucose were added as carbon source as indicated. The medium was supplemented with 
auxotrophic requirements (at $50 \mathrm{mg} / \mathrm{l}$ ). E. coli was grown in LB medium and transformants were selected on plates containing ampicillin $(100 \mu \mathrm{g} / \mathrm{ml})$. LB, SP and CSE plates were prepared by the addition of $17 \mathrm{~g}$ Bacto agar/l (Difco, Lawrence, USA) to LB, SP or CSE medium, respectively.

\section{DNA manipulation and transformation}

Transformation of E. coli and plasmid DNA extraction were performed using standard procedures (Sambrook et al., 1989). Restriction enzymes, T4 DNA ligase and DNA polymerases were used as recommended by the manufacturers. DNA fragments were purified from agarose gels using the Nucleospin Extract kit (Macherey and Nagel, Düren, Germany). Phusion ${ }^{\mathrm{TM}}$ DNA polymerase was used for the polymerase chain reaction as recommended by the manufacturer (New England Biolabs, Frankfurt am Main, Germany). All primer sequences are provided as supplementary material (Tab. S2). DNA sequences were determined using the dideoxy chain termination method (Sambrook et al., 1989). All plasmid inserts derived from PCR products were verified by DNA sequencing. Chromosomal DNA of B. subtilis was isolated as described (Kunst \& Rapoport, 1995).

E. coli transformants were selected on LB plates containing ampicillin (100 $\mu \mathrm{g} / \mathrm{ml})$. B. subtilis was transformed with plasmid DNA or PCR products according to the two-step protocol described previously (Kunst \& Rapoport, 1995). Transformants were selected on SP plates containing tetracycline (Tet $10 \mu \mathrm{g} / \mathrm{ml}$ ), or erythromycin plus lincomycin (Erm $2 \mu \mathrm{g} / \mathrm{ml}$ and Lin $25 \mu \mathrm{g} / \mathrm{ml})$.

\section{Plasmid and mutant strain construction}

To express a plasmid-borne ansR gene in B. subtilis, we constructed plasmid pGP873. For this purpose the ansR gene was amplified with the primers KG18 and KG19 using chromosomal DNA of B. subtilis as a template (all primer sequences are provided in Tab. S1). The PCR product was digested with BamHI and SalI and cloned into the overexpression vector $\mathrm{pBQ} 200$ (Martin-Verstraete et al., 1994).

Deletion of the ansAB and ansR genes was achieved by transformation with PCR products constructed using oligonucleotides to amplify DNA fragments flanking the target genes and an intervening erythromycin and tetracycline resistance cassettes from 
plasmids pDG647 and pDG1514, respectively (Guérout-Fleury et al., 1995), as described previously (Wach, 1996). The PCR products were used to transform GP717 and GP28 for the deletion of the ansAB and ansR, respectively.

\section{Reverse transcription-real-time quantitative PCR}

For RNA isolation, the cells were grown to an $\mathrm{OD}_{600}$ of $0.5-0.8$ and harvested. Preparation of total RNA was carried out as described previously (Ludwig et al., 2001). cDNAs were synthesized using the One-Step RT-PCR kit (BioRad, Munich, Germany) as described (Rietkötter et al., 2008). Real time quantitative PCR was carried out on the iCycler instrument (BioRad, Munich, Germany) following the manufacturer's recommended protocol by using the primers KG26/KG27 for the ansA gene, KG38/KG39 for the ald gene and KG40/KG41 for the bcd gene, respectively. Their recommended data analysis procedure was also used. The rpsE and rpsJ genes encoding constitutively expressed ribosomal proteins were used as internal controls and were amplified with the primers rpsE-RT-fwd/rpsE-RT-rev and rpsJ-RT-fwd/rpsJ-RT-rev, respectively. The expression ratios were calculated as fold changes as described (Rietkötter et al., 2008). RT-PCR experiments were performed in duplicate. 


\section{Results}

\section{Isolation of a mutation that allows a bypass of the glutamate dehydrogenase for the utilization of glutamate}

Glutamate is the most abundant metabolite in a bacterial cell. Although its exact concentration in B. subtilis is unknown, it is known to account for about $40 \%$ of the internal metabolite pool of an Escherichia coli cell (Yuan et al., 2009). Glutamate serves as an osmotic regulator (Whatmore et al., 1990), as well as universal amino group donor in anabolism thus linking carbon and nitrogen metabolism (Commichau et al., 2006). In B. subtilis, at least 37 reactions make use of glutamate as cofactor for transamination (Oh et al., 2007).

The key reactions of glutamate biosynthesis and degradation in B. subtilis are summarized in Fig. 6.1 2-oxoglutarate, an intermediate of the citric acid cycle, is aminated by the glutamate synthase, encoded by the gltA and gltB genes. Glutamate degradation to 2-oxoglutarate requires the glutamate dehydrogenase RocG. Additionally, the laboratory strain $B$. subtilis 168 harbors a cryptic gene, gudB, coding for an inactive glutamate dehydrogenase. This gene is readily decryptified in $\operatorname{roc} G$ mutants (Belitsky \& Sonenshein, 1998; Commichau et al., 2008). In addition, RocG controls the expression of the gltAB operon and therefore prevents glutamate biosynthesis in the presence of arginine (Belitsky \& Sonenshein, 2004; Commichau et al., 2007b). 


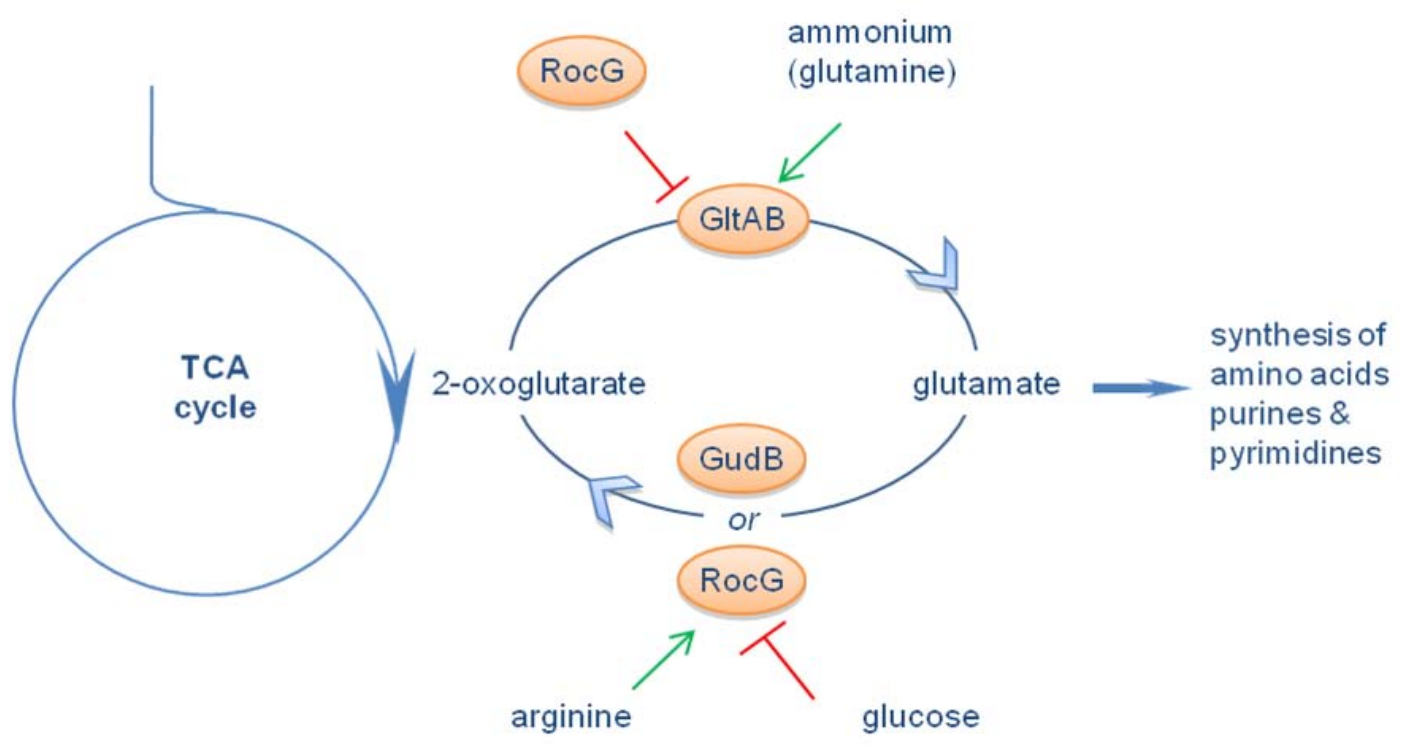

Fig. 6.1 - Key reactions for glutamate biosynthesis and degradation in B. subtilis.

Glutamate is the universal amino group donor in all living cells and in that way links the carbon and nitrogen metabolisms. In $B$. subtilis the synthesis of glutamate depends on the glutamate synthase GltAB. In addition, the genome encodes two glutamate dehydrogenases, RocG and GudB, although the latter is inactive in the laboratory B. subtilis strain 168 (see text). The synthesis and degradation of glutamate are tightly regulated in response to the availability of carbon and nitrogen sources.

Inactivation of both the $\operatorname{roc} G$ and the $g u d B$ gene results in loss of any glutamate dehydrogenase activity and concomitant inability of the bacteria to utilize glutamate (Belitsky \& Sonenshein, 1998; Commichau et al., 2008). The rocG gudB double mutant strain GP28 grows poorly on SP medium (an amino acid-rich medium) due to the accumulation of degradation products of arginine metabolism (Commichau et al., 2007a). However, cultivation of GP28 on SP plates eventually resulted in the isolation of a mutant (GP717) that carries a mutation inactivating the gltB gene, encoding a subunit of the glutamate synthase (Commichau et al., 2008). This gltB1 mutation leads to glutamate auxotrophy and might therefore prevent the accumulation of intermediates of arginine degradation. We have observed that toxic intermediates of arginine degradation result in poor growth of mutants lacking a functional glutamate dehydrogenase (our unpublished results). If intrinsic glutamate synthesis is blocked by a mutation, such an accumulation of toxic intermediates might be reduced. A careful analysis of the mutant strain revealed that it had acquired the ability to utilize glutamate 
as the only source of carbon and energy. This might have resulted from a re-activation of the $\operatorname{roc} G$ or $g u d B$ genes or from the establishment of a novel pathway for glutamate utilization. We tested therefore the $\operatorname{roc} G$ and $g u d B$ alleles by PCR analysis. Both the transposon insertion in $\operatorname{roc} G$ and the replacement of the $\operatorname{gudB}$ gene by a chloramphenicol resistance gene were identical to the parent strain GP28. Clearly, a new pathway of glutamate degradation was activated in this suppressor mutant that was not active in the wild type and $\operatorname{roc} G$ gudB mutant cells.

\section{Development of a pathway-finding algorithm}

The most reasonable hypothesis to explain the suppression was that the mutation had activated a redundant pathway that is inactive in the wild type strain in a medium with glutamate as single carbon source. Since glutamate is a highly abundant metabolite and is involved as a substrate in 20 reactions in B. subtilis, it was not obvious which mutation could have lead to glutamate utilization proficiency in B. subtilis GP717.

To address this problem by use of the power of bioinformatics, we developed an approach that harnesses the strengths of Boolean satisfiability (SAT) to find valid pathways (see Materials and Methods). It is able to find short pathways between a basis and a target set (SPABBATS) of metabolites that can operate in a sustained way. It is convenient for its focus on short pathways and the fact that it can calculate pathways that comply with the steady-state constraint. It also allows the relaxation of this constraint, by allowing some metabolites to accumulate if necessary. 
A
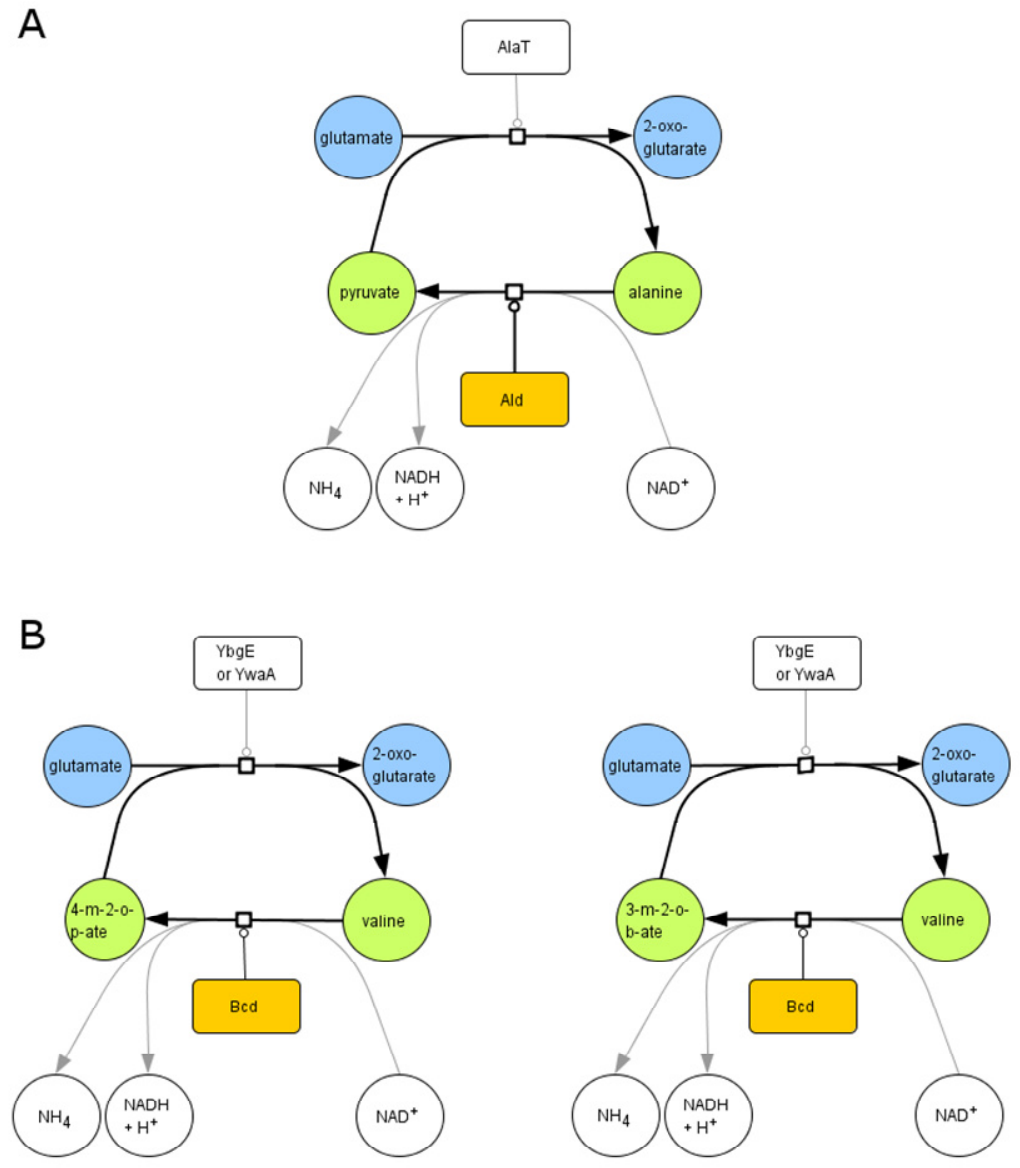

C

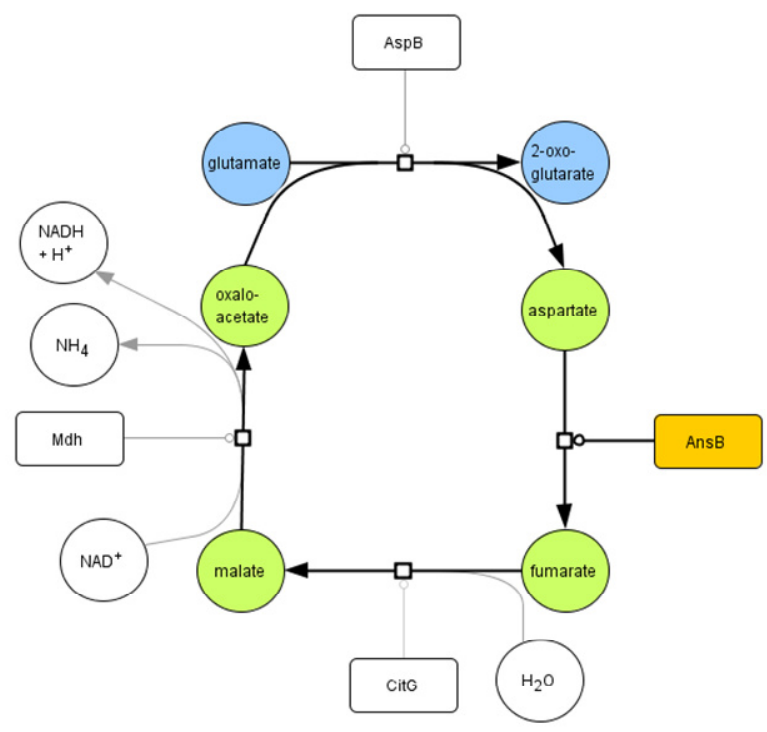

Figure 6.2 - Predictions of alternative pathways for glutamate utilization based on SAT techniques.

Fig. legend see next page. 
Figure 6.2 - Predictions of alternative pathways for glutamate utilization based on SAT techniques.

A B. subtilis strain (GP28) was constructed that lacks the glutamate dehydrogenases. An evolutionary adaptation resulted in a strain (GP717) that acquired the capacity to grow on glutamate as single carbon source. Using a SAT based search algorithm (see Materials and Methods) we predicted four alternative pathways that could be activated in the GP717. The genes coding for the enzymes in orange were analyzed further (see text). 4-m-2-o-p-ate $=4$-methyl-2-oxo-pentanoate; 3-m-2-o-b-ate $=3$-methyl-2-oxobutanoate.

The first four pathways suggested by our algorithm are presented in Fig. 6.2 In each case, the first step is a transamination reaction that leads to the production of 2oxoglutarate. The substrate for transamination is then replenished via the remaining reaction(s) of the pathway. The first pathway (Fig. 6.2A) involves transamination to form alanine and subsequent oxidative deamination of alanine by the alanine dehydrogenase Ald resulting in the net formation of 2-oxoglutarate. The next two pathways (Fig. 6.2B) are very similar and involve enzymes of branched amino acid metabolism. In the transamination step, both pathways use the transaminases YbgE and YwaA. The branched chain amino acid dehydrogenase Bcd is then used for the oxidative deamination of the transamination products valine or leucine. Again, the net result of this pathway is the production of 2-oxoglutarate from glutamate. The last pathway (Fig. 6.2C) requires four steps, (i) the reaction of the aspartate aminotransferase AspB, (ii) the deamination of asparate to fumarate by the aspartase AnsB, (iii) the fumarase reaction (CitG) of the citric acid cycle, and finally (iv) the oxidation of malate by the malate dehydrogenase Mdh. As described for the other pathways, this reaction sequence results in the net formation of 2-oxoglutarate from glutamate. Since the original mutant GP28 did not grow with glutamate as the single carbon source, it is obviously not able to use any of these proposed pathways suggesting that they were activated by a suppressor mutation in GP717.

\section{Experimental validation of the predictions}

Our experiments were performed in minimal medium suggesting that the activity of transaminases was not limiting. Similarly, the two enzymes of the citric acid cycle (CitG and Mdh) are constitutively expressed (Jin \& Sonenshein, 1994; Feavers et al., 1998; Blencke et al., 2003). Thus, the mutation may have affected the expression of one 
of the deaminases Ald, Bcd or AnsB. This hypothesis was tested by reverse transcription-real-time quantitative PCR. As shown in Fig. 6.3, the levels of ald and $b c d$ mRNA are comparable for the original mutant GP28 and the suppressor strain GP717. In contrast, a strong increase of the expression of the ans $A B$ operon encoding the asparaginase and aspartase was observed for the suppressor mutant that was able to utilize glutamate. This observation suggests that it is the high-level expression of AnsB that allows glutamate utilization in GP717.

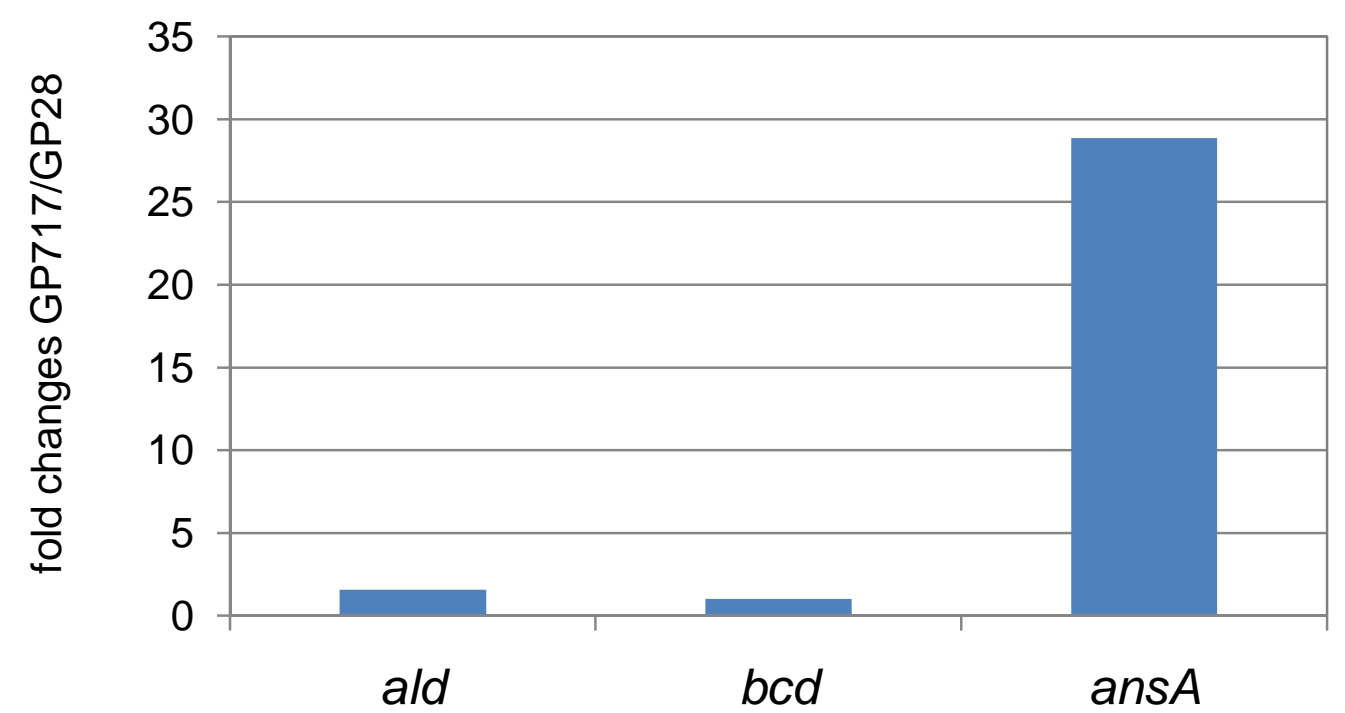

Fig. 6.3 - Comparison of gene expression patterns between mutant and parental strains, based on the predictions of the SPABBATS algorithm for pathway analysis.

The predictions of the SPABBATS algorithm (see Fig. 6.2) were further characterized by transcription analysis. The expression of the ald and $b c d$ genes remains constant between the mutant (GP717) and parental (GP28) strains, suggesting that these genes are not involved in the newly activated catabolic pathway. In contrast, the expression of the ans $A B$ operon is strongly increased in the mutant. This hints to a gain of function in the mutant strain that was analyzed further. 

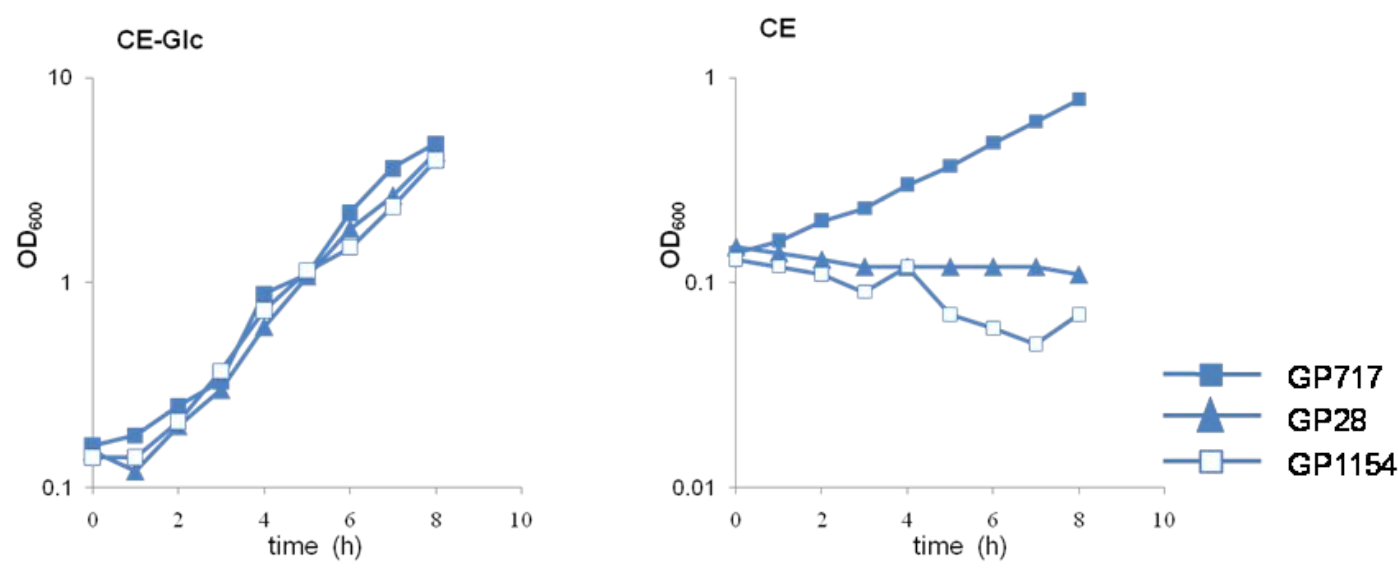

Fig. 6.4 - Requirement of the aspartase gene in the alternative pathway for glutamate utilization.

The SPABBATS algorithm (see Fig. 6.2) and the transcription analysis (see Fig. 6.3) suggested that the overexpression of the asparaginase and aspartase genes (ans $A B)$ is the cause for the metabolic gain of function of the mutant strain GP717. To prove this, the ansAB operon was deleted in the GP717 strain. The resulting strain GP1154 lost the capacity to utilize glutamate as single carbon source. This strongly indicates that the induction of the aspartase gene is required and sufficient for the newly activated catabolic pathway. $\mathrm{CE}=$ Minimal medium containing $8 \mathrm{~g} / \mathrm{l}$ glutamate, $\mathrm{CE}-\mathrm{Glc}=\mathrm{CE}$ medium with an addition $5 \mathrm{~g} / \mathrm{l}$ glucose.

The involvement of the aspartase AnsB in the novel glutamate utilization pathway was verified by analyzing the effect of a deletion of the ans $A B$ operon. Growth of the original strain GP28, the suppressor mutant GP717 and its isogenic $\triangle a n s A B$ mutant derivative GP1154 in minimal medium with glutamate or with glutamate and glucose was recorded. As shown in Fig. 6.4, all three strains were able to grow with glutamate and glucose. In contrast, the deletion of the ans $A B$ operon reverted the capability of the suppressor strain of using glutamate as the single carbon source, and the $\triangle a n s A B$ mutant GP1154 was unable to grow with glutamate as was the original strain GP28. This finding strongly supports the idea that the activity of the aspartase AnsB is the reason for the ability of the suppressor strain GP717 to utilize glutamate.

The ans $A B$ operon is induced in the presence of asparagine due to inactivation of the AnsR repressor (Sun \& Setlow, 1991; Sun \& Setlow, 1993; Fisher \& Wray, 2002). A comparative analysis of $a n s A B$ expression revealed about 30-fold induction by asparagine in GP28, whereas the expression levels were unaffected by the availability of asparagine in the suppressor mutant GP717 (data not shown). The observed induction in 
the wild type strain is in good agreement with previous reports. The loss of regulation in GP717 and the high expression of the operon as compared to GP28 suggest constitutive $a n s A B$ expression that might be the result of an inactivation of the ans $R$ repressor gene.

To test the hypothesis that inactivation of the AnsR repressor allowed glutamate utilization by GP717, we performed two tests: First, we deleted the ansR gene of the parental strain GP28 and tested the ability of the resulting strain GP811 to grow with glutamate as the single carbon source. Unlike GP28, this strain GP811 ( $\Delta$ ansR) grew in $\mathrm{CE}$ minimal medium. Thus, inactivation of the ans $R$ gene is sufficient to open a new pathway for glutamate catabolism. In a complementary approach, we complemented B. subtilis GP717 with a plasmid-borne copy of the ansR gene (present on pGP873) and tested the ability of the transformants to use glutamate. While the control strain (GP717 transformed with the empty vector $\mathrm{pBQ} 200$ ) grew well on CE medium, expression of AnsR from the plasmid completely blocked growth in this medium, i.e. the utilization of glutamate. This result confirms that a mutation in the ans $R$ gene must be present in GP717 and that it is this mutation, which confers the bacteria with the ability to utilize glutamate via the new aspartase pathway.

To identify the mutation in ansR, we sequenced the ansR alleles of the parental strain GP28 and the glutamate-utilizing suppressor mutant GP717. While the wild type allele of ans $R$ was present in GP28, a C-to-A substitution at position 107 of the ansR open reading frame was found in GP717. This mutation changes codon 36 from UCA (Ser) to UAA (stop) and results in premature translation termination and the formation of an incomplete and non-functional AnsR repressor protein.

Taken together, these experiments confirmed that the metabolic pathway predicted by the SPABBATS algorithm corresponds to a valid metabolic state of the rocG gudB ansR mutant strain GP717. 


\section{Discussion}

\section{Comparison of SPABBATS with other methods for metabolic analysis}

Flux balance analysis and the majority of methods derived from it are based on constraining the admissible intracellular flux space to steady-state and choosing an adequate optimality criterion to calculate intracellular fluxes (Orth et al., 2010). Commonly used optimization criteria are biomass production and the maximization of energy output.

Although these methods predict the essentiality of genes with high accuracy (Oh et al., 2007), they are less suited for the characterization of alternative metabolic pathways in viable mutants. On the one hand, by restricting the admissible intracellular flux to steady-state, they discard pathways where a by-product accumulates. Nonetheless, the cell is still viable if this by-product is consumed by other pathways in the cell, not directly related to the process that is studied. SPABBATS solves this problem by allowing a larger flux-space, where intermediate products can accumulate, if necessary.

On the other hand, the optimality criterion can be artificial. For instance, maximizing cellular growth might lead to a theoretical maximum growth rate, or a flux distribution that is as close to the wild-type flux as possible, but it is hard to argue that the regulatory network of the strain is directed to the same target. The pathways discovered by SPABBATS are a structural property of the network and do not depend on an extrinsic optimality criterion (beyond the number of reactions of the resulting pathway). For this reason, the resulting pathways can be interpreted objectively.

Other methods for structural decomposition (e.g. extreme pathways and elementary flux modes, [Planes \& Beasley, 2008]) rely on the same steady-state restriction of FBA related methods and for this reason share some of their disadvantages. Moreover, SPABBATS does not require the calculation of all possible pathways. Instead, it can be used iteratively to calculate pathways of increasing length, which results in a dramatic improvement in performance for finding relevant pathways in large networks.

An advantage over the method of de Figueiredo et al. (de Figueiredo et al.) is that we do not make use of an optimization framework, but select for satisfiability instead. Similar problems in other areas of computational biology (e.g. Graça et al., 2007) show 
a performance improvement of SAT methods over traditional mixed-integer linear programming methods.

\section{Future perspectives}

So far, our analysis of networks using SAT has been restricted to metabolic networks. Nonetheless, since SAT is especially suited for problems that involve Boolean constraints, it is possible to expand the analysis to regulatory networks. For B. subtilis, this implies the reconstruction of the metabolic network together with its regulatory complement. This reconstruction is in progress (Goelzer et al., 2008; Lammers et al., 2010).

In parallel, we envision the development of novel SAT solvers that are optimized for the solution of metabolic constraints. This will result in the adoption of SAT based methods for metabolic engineering as well as for the design of synthetic circuits that are able to perform computations in the same way as their silicon-made counterparts (Lou et al., 2010).

\section{Conclusions}

In this contribution we have shown the use of SAT techniques to discover alternative pathways that connect sets of starting and target species. In addition, we provided a proof of concept for the applicability of the algorithm. We started with a complex physiological problem in B. subtilis: the need to characterize a suppressor mutation that allowed growth on glutamate without glutamate dehydrogenases. SPABBATS predicted four potential pathways for glutamate utilization that were decisive to suggest target genes for experimentation. These experiments confirmed the validity of the SPABBATS' prediction, closing the cycle between modelling and wet lab experimentation.

SPABBATS relies on Boolean satisfiability (SAT) to construct the metabolic pathways. SAT has been used for the determination of haplotypes from sequenced genotypes (Graça et al., 2007), the analysis of genome biology networks (Chin et al., 2008), the understanding of myogenic differentiation (Piran et al., 2009), and the characterization of steady states of regulatory circuits (Tiwari et al., 2007; de Jong \& 
Page, 2008). Here we report the first application of SAT techniques to metabolic problems.

The SPABBATS algorithm was applied here to a specific problem, the analysis of glutamate metabolism in B. subtilis. However, the solution strategies are applicable to a broad spectrum of metabolic problems. For instance, SPABBATS can be particularly useful in the characterization of suppressor mutants. Moreover, SPABBATS can also be useful in synthetic biology. Although used here to find pathways in a reconstruction of the metabolism of $B$. subtilis, it is also possible to use a database of enzymes as the starting model. In this way, it can be used to construct synthetic pathways that satisfy specific input-output and mass-balance requirements.

\section{Acknowledgements}

We are grateful to Jens Baumbach for the help with some experiments and to Fabian M. Commichau and Jens J. Landmann for helpful discussions. Christine Diethmaier and Sina Jordan are acknowledged for introducing us to RT-PCR. L. A. F. was supported by the International Molecular Biology Program of the University of Göttingen and the Studienförderwerk Klaus Murmann der Stiftung der Deutschen Wirtschaft. R. P. was supported by a stipend of the Rose Foundation. This work was supported by the Federal Ministry of Education (Research SYSMO network (PtJBIO/0313978D)) and the Fonds der Chemischen Industrie to J. S. 


\section{Discussion}

\subsection{The emergence of suppressor mutations in response to glutamate imbalance}

In the absence of the active GDH RocG in B. subtilis, the cryptic GDH GudB1 is rapidly decryptified upon growth on complex medium. The rate of this gudB1 mutation is $10^{-4}$ (Gunka et al., subm.). Compared to rates of beneficial mutations in bacteria that are in a range of $10^{-9}$ the $g u d B$ decryptification occurs with an extremely high rate (Imhof \& Schlötterer, 2001). The gain of function mutation in the $\operatorname{gudB}$ gene allows a $\operatorname{roc} G$ mutant to degrade glutamate and to overcome the severe growth defect of this mutant (see Fig. 1.3).

Previous results revealed that a $\operatorname{roc} G \operatorname{gudB}$ double mutant can also accumulate suppressor mutations that facilitate good growth of this mutant when incubated on complex medium (Commichau et al., 2008). One mutation was found to inactivate the GOGAT leading to the loss of glutamate synthesis in this mutant. Furthermore, this strain had acquired a second suppressor mutation that allows the utilization of glutamate as carbon source even in the absence of a glutamate dehydrogenase (Flórez et al., subm.). This mutation is located in the gene for the repressor AnsR of the aspartase/asparaginase operon ansAB (Sun \& Setlow, 1991). A nonesense mutation inactivated the repressor AnsR resulting in a constitutive expression of the ans $A B$ operon. This alternative pathway was discovered by using a Boolean satisfiability (SAT) method that constructs the shortest pathway between the basal and target sets (SPABBATS) of metabolites that is stoichiometrically balanced (Flórez et al., subm.). Both mutations, the inactivation of the GOGAT and the activation of an alternative pathway for the glutamate degradation, probably result in a decrease of the glutamate level of the cell. It was also reported for Enterobacteria that the perturbation of glutamate homeostasis results in the accumulation of suppressor mutations that balance the glutamine to glutamate ratio to wild type level (Yan, 2007). Taken together with the gudB1 suppressor mutation, it is tempting to speculate that the accumulation of glutamate might be highly problematic for B. subtilis, although glutamate is the most abundant molecule in the cell anyway. The complex medium contains beef extract and is therefore composed of a variety of nitrogen containing compounds such as arginine 
and glutamate but the medium lacks sugars. A $\operatorname{roc} G$ mutant is not capable of converting glutamate to 2-oxoglutarate. Perhaps the lack of glutamate degradation is disadvantageous upon cultivation on complex medium and might lead to a decrease in TCA intermediates. By the activation of GudB this bottleneck might be resolved. Another possibility that could explain the growth defect of a $\operatorname{roc} G$ mutant is that toxic metabolites accumulate in the cell in the absence of an active GDH.

The enzyme glutamate racemase (encoded by the essential gene racE in B. subtilis) catalyzes the conversion of L-glutamate to D-glutamate that is a building block for peptidoglycan biosynthesis (Kimura et al., 2004; Spies et al., 2009). Indeed, the accumulation D-glutamate was shown to be toxic for B. subtilis (Kimura et al., 2004). In the presence of very high intracellular amounts of L-glutamate due to the strong induction of the enzymes of the arginine degradation pathway, RacE probably generates higher concentrations of D-glutamate than tolerated by the cell. The activation of the cryptic glutamate dehydrogenase $\mathrm{GudB}$, the initialization of an alternative pathway to degrade glutamate and the inactivation of the GOGAT might then bring the glutamate concentration to a level that does not longer result in the accumulation of harmful D-glutamate. This hypothesis has to be subject of further investigation.

Additionally to the suppression of the growth defect on complex medium, the ans $R$ mutation is also sufficient to provide growth of this mutant strain with glutamate as single carbon source (Flórez et al., subm.). The gudB1 mutation also facilitates the growth of B. subtilis with glutamate as sole source of carbon (Commichau et al., 2008). Interestingly, the ansR mutant strain was isolated on complex medium as described. Thus, the activation of the alternative pathway for glutamate degradation is not only beneficial for the growth of this mutant on rich medium but is also essential for a condition to which the mutant was not exposed during isolation.

\subsection{The cryptification of the gudB gene}

It is well established that cellular processes are highly regulated. The expression of genes required for the utilization of a nutritional source is induced as a response to the availability of this particular source. Moreover, enzymes for the biosynthesis of a certain metabolite are only expressed when the metabolite is limiting. Actually, the accumulation of a metabolite often represses the transcription of genes, needed for its 
synthesis (Sonenshein, 2007). This regulation guarantees the utilization of a particular compound and the supply with a particular metabolite at a defined point of time. This can be regarded as efficiency, since a waste of energy and resources is prevented.

In this context the presence and most notably the expression of the cryptic glutamate dehydrogenase seem paradoxical in $B$. subtilis. This inactive enzyme is highly expressed even in the absence of its substrate glutamate and moreover it is rapidly degraded (Chapter 4; Gerth et al., 2008). An in depth analysis of a variety of laboratory $B$. subtilis strains and wild type isolates revealed that a functional $g u d B$ allele naturally occurs in the two wild Marburg strains as well as in Burkholder and Giles strain 122 (Zeigler et al., 2008). The presence of a functional gudB allele in the ancestors of the laboratory $B$. subtilis strains has two implications. First, the domestication of $B$. subtilis might have caused the $g u d B$ cryptification and second, the active GudB enzyme might be the main glutamate dehydrogenase in wild type isolates.

It is well established that the cultivation in the laboratory can have a striking impact on bacteria. B. subtilis wild type isolates form highly differentiated multicellular communities whereas the laboratory strain can only form thin and relatively undifferentiated biofilms (Branda et al., 2001; Kearns \& Losick, 2003). In the B. subtilis strain 168, not only biofilm formation is affected but also the swarming motility. The strain 168 is unable to swarm due to a frameshift mutation in the swrA gene (Patrick \& Kearns, 2009).

Interestingly, cultivation of bacteria in the laboratory does not always lead to loss of function mutations but also to gain of function mutations. In naturally occurring E. coli strains the $b g l$ operon is cryptic. Under selective conditions in the laboratory, the transcription of the $b g l$ operon is enhanced by the acquisition of a mutation and this enables $E$. coli cells to utilize $\beta$-glucosides (Hall \& Betts, 1987). It is tempting to speculate that cryptic genes might encode unusual functions that can be activated upon selective pressure (Tamburini \& Mastromei, 2000).

The hypothesis that GudB is the original enzyme for glutamate degradation is not only supported by the fact that GudB is an active glutamate dehydrogenase in wild type isolates of B. subtilis. Moreover, the expression of the glutamate dehydrogenase RocG, the active enzyme in $B$. subtilis 168 , is not induced by its substrate glutamate but by arginine, ornithine and citrulline as indicated by its name, RocG for the abbreviations of arginine (R), ornithine (o), citrulline (c) (Belitsky \& Sonenshein, 1998). Moreover, the 
$\operatorname{roc} G$ gene is chromosomally clustered with other genes which are necessary for arginine utilization (RocABC). These observations might support the idea that GudB is responsible for glutamate utilization and RocG is needed when precursors of glutamate are available. In biochemical analyses the enzymatic parameters of RocG and GudB1 were determined (see Chapter 2). The GudB1 protein exhibits a higher enzymatic activity in carrying out glutamate degradation than the RocG protein $(6.7 \mathrm{U} / \mathrm{mg}$ of protein and $3.9 \mathrm{U} / \mathrm{mg}$ of protein, respectively). In contrast, the GudB1 protein shows a lower affinity for its substrate glutamate compared to RocG ( $\mathrm{k}_{\mathrm{m}}$ values of $17.9 \mathrm{mM}$ and $2.9 \mathrm{mM}$, respectively). These data might support the following idea: During glutamate excess, GudB1 is relatively active in degrading glutamate and therefore supplies the cell with 2-oxoglutarate. Subsequently, 2-oxoglutarate can be used in the TCA cycle for energy supply and other biosynthetic steps. But if glutamate is limiting in the cell, the high $\mathrm{k}_{\mathrm{m}}$ value for glutamate might prevent that the low amount of glutamate is readily catabolized by GudB1, which is constitutively expressed irrespective of glutamate availability. The higher affinity of the RocG protein for glutamate might support growth with precursors of glutamate e. g. arginine. Due to its biochemical properties RocG might carry out glutamate degradation already at low glutamate concentrations.

The question remains why the cryptic gudB allele is highly stable in B. subtilis 168 upon cultivation in the laboratory but is so readily decryptified upon growth on complex medium in a $\operatorname{roc} G$ mutant. It is possible that both glutamate dehydrogenases are redundant upon cultivation in the laboratory, as the medium contains all nutrients required for growth, implying that the presence of RocG is sufficient to support growth on the medium B. subtilis is faced in the lab. But the absence of RocG is supposed to provoke a fast reactivation of GudB on complex medium as well as upon growth with glutamate as single carbon source (Belitsky \& Sonenshein, 1998; Commichau et al., 2008).

Based on the fact that the $g u d B$ allele is not cryptic in the ancestors of B. subtilis 168 , it should be taken into account to rename the allele in the 168 strain. It would be easier to understand to use $g u d B$ for the functional gene and $g u d B 1$ for the cryptic gene in B. subtilis. 


\subsection{The Mfd protein is required for the $g u d B$ decryptification}

The work revealed that the gudB1 mutation occurs with a rate of $10^{-4}$. To the best of our knowledge, this is an extremely high mutation rate not only in B. subtilis. A striking feature of the $g u d B$ allele is the direct repeat of nine base pairs. Mutational analysis of the nine bp direct repeat showed that a perfect direct repeat is essential for the high decryptification rate of the $g u d B$ allele (see Chapter 4). It is well established that repeated sequences are common in a variety of genomes. This feature is highly abundant in particular in eukaryotic genomes but was also found to be a major factor for genomic rearrangement in bacteria (Viguera et al., 2001; Michel, 2000). The instability of tandem repeats is supposed to be linked with replication. If the DNA polymerase is arrested at a repeated DNA sequence a deletion or an expansion of the repeated sequence can occur. The arrest of the DNA polymerase at the first part of the repeat can lead to a deletion whereas the arrest to the second part might result in an expansion (Michel, 2000). This model was termed as replication slippage. Especially tandem repeats are highly unstable and often undergo a contraction as well as an expansion (Bichara et al., 2006). Well-studied tandem repeats such as the $\operatorname{lgtC}$ repeat in Haemophilus influenzae or the nadA repeat in Neisseria meningitidis consist of five to 36 repeats of tetranucleotides (Bayliss et al., 2001; Martin et al, 2005). In those cases, the instability is linked to replication rather than to transcription (Bichara et al., 2006). The $g u d B$ repeat is unique in possessing a large repeat (unit repeat of nine nucleotides) but this sequence is only repeated in two units. Compared to the length of well characterized repeats, two units are relatively short.

If a replication slippage mechanism would be the driving force for the rapid $\operatorname{gudB}$ decryptification, we would have expected to obtain the same high mutation rate of this repeat in a non-related genomic context. However, the use of the artificial mutagenesis promoter system revealed that the deletion of the nine bp of the direct repeat can occur in a different context but the mutation rate was reduced by three orders of magnitude. The artificial promoter system is located in a nontranscribed genomic region. Therefore, only replication can be involved in the emergence of mutations. This implies that the high mutation rate of the $g u d B$ allele needs another pathway than replication that can generate mutations. Indeed, the transcription-repair coupling factor Mfd turned out to be required for the high mutation rate of the $g u d B$ allele (see Chapter 4). In a $m f d$ deficient background the mutation rate of the $g u d B$ allele was reduced hundred fold. Mfd 
mediates the transcription-coupled DNA repair that is induced by the arrest of the RNA polymerase at a DNA lesion during transcription. Mfd displaces the RNA polymerase and recruits the nucleotide excision repair system to solve the lesion (Roberts \& Park, 2004). Other studies have suggested that Mfd is necessary for the accumulation of mutations in transcribed genes during the stationary phase in B. subtilis (Ross et al., 2006; Pybus et al., 2010). The Mfd-mediated coupling of transcription to DNA repair and might facilitate the accumulation of mutations preferentially in transcribed genes. This has several implications: (i) The coupling allows that the mutations occur in genes that are expressed at the given time point; therefore the mutant variants of the encoded proteins might help to overcome the actual limitation. (ii) Non-transcribed genes that may be required under different conditions are in this way protected from potentially harmful mutations. Both effects facilitate the adaptation of bacteria to all kind of challenges that limit their growth and are therefore crucial for bacterial evolution. As shown, the $g u d B$ allele is highly expressed. Thus, the $g u d B$ allele fulfills the prerequisite to be subject of transcription-coupled DNA repair.

Furthermore, this work addressed the question which DNA repair system acts in concert with the Mfd protein in the $\operatorname{gudB}$ decryptification. The loss of the UvrABC as well as the MutSL system does not decrease the gudB mutation rate (see Chapter 5). Even the simultaneous deletion of the $u v r A B$ and the mutSL genes does not influence the $\operatorname{gudB}$ decryptification. Therefore, the repair system that operates with the Mfd protein in the $g u d B 1$ mutation has not been identified yet and might be subject to further investigation.

\subsection{A perfect direct repeat is required for the efficient gudB decryptification}

Evidence was provided that a perfect direct repeat is crucial for the high mutation rate of the $g u d B$ allele. Destroying the direct repeat by introducing mutations in the first half as well as in the second half led to a fifteen fold decrease in the mutation rate and also the occurrence of $g u d B 1$ revertants upon long time incubation on complex medium was significantly diminished (our unpublished data) These results emphasize the importance of the nine bp direct repeat for the rapid decryptification of the $g u d B$ gene. Since compensatory mutations in both parts of the direct repeat did not decrease the 
mutation frequency, the wild type sequence of the direct repeat is not necessary for the high mutation rate.

As shown, the process of transcription coupled DNA repair is involved in the decryptification of $g u d B$. Therefore, it is important whether the $g u d B$ sequence exhibits a particular feature that could cause the arrest of the RNA polymerase during transcription. One feature could be the formation of secondary structures in the coding strand.

An analysis of the direct repeat DNA sequence in the genomic context of the $\operatorname{gudB}$ allele in the transcribed strand (30 bases) with the mfold web server revealed a putative secondary structure (see Fig. 7.1) (Zucker, 2003). The predicted structure has a free energy of $-2.85 \mathrm{kcal} / \mathrm{mol}$ (see Fig.7.1A). This secondary structure might be involved in the Mfd-mediated mutation in the $\operatorname{gudB}$ allele. During transcription the DNA is single stranded which might favor the formation of secondary structures. The replacement of two $G$ residues by $T$ in the first and the second half of the repeat (position 3 and 9 of the repeat) leads to a fifteen fold decrease of mutation frequency. This might correlate to a weaker secondary structure formation of these sequences. The free energy of putative secondary structures of these sequences is $-0.88 \mathrm{kcal} / \mathrm{mol}$ for the mutations in the first half and $-0.65 \mathrm{kcal} / \mathrm{mol}$ for the mutations in the second part of the direct repeat (see Fig. 7.1BC). A weaker secondary structure might reduce the chance of a roadblock during transcription. Moreover, the use of a non perfect direct repeat allowed us to address the question which half of the direct repeat has been excised. It turned out that the first part of the direct repeat is preferentially excised. However, in the minority of the sequenced mutants an internal deletion occurred (see chapter 4). Restoring the perfect direct repeat with $\mathrm{T}$ substitutions in both parts of the direct repeat leads to a mutation rate that is in the range of the wild type rate. Indeed, the calculated energy for a secondary structure of this DNA sequence is roughly comparable to the wild type structure (see Fig. 7.1.A and Fig. 7.1.D; $-2.43 \mathrm{kcal} / \mathrm{mol}$ for the mutant perfect repeat and $-2.85 \mathrm{kcal} / \mathrm{mol}$ for the wild type perfect repeat, respectively). 


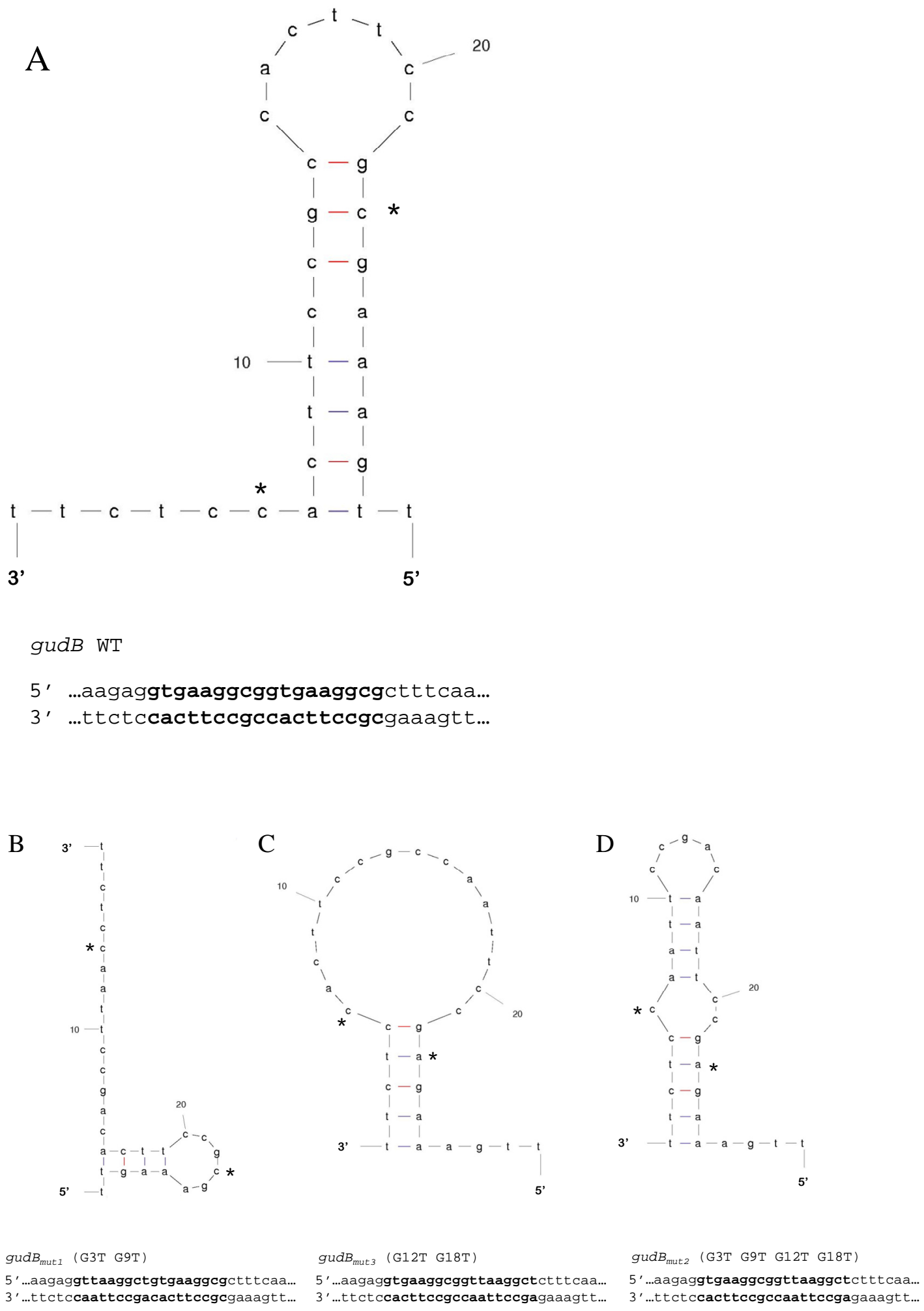

Fig. 7.1 - Putative DNA secondary structures of the gudB direct repeat and its mutant derivatives.

Fig. legend see next page. 


\section{Fig. 7.1 - Predicted DNA secondary structures of the gudB direct repeat and its mutant derivatives.}

For the secondary structure prediction the mfold web server was used (Zucker, 2003). As the template for prediction 30 bases of the coding strand were used containing the direct repeat. The first and the last base of the direct repeat are marked with an asterisk. The DNA sequence used for prediction is shown above the structure and the repeat is indicated by bold letters (A). The calculated free energy of the structure for the wild type sequence is $-2.85 \mathrm{kcal} / \mathrm{mol}$. Predictions of the secondary structures of the imperfect direct repeats are (B) $-0.88 \mathrm{kcal} / \mathrm{mol}$ and (C) $-0.65 \mathrm{kcal} / \mathrm{mol}$. (D) Recovery ofthe direct repeat with the identical substitutions in the first and the second half results in a predicted free energy of $-2.43 \mathrm{kcal} / \mathrm{mol}$.

This result implies that the deletion might depend on a stable secondary structure rather than on the wild type sequence. The secondary structure prediction might support the idea that the rapid decryptification of the $g u d B$ allele is induced by an RNA polymerase arrest caused by a DNA lesion in the coding strand and requires the Mfd protein.

The formation of secondary structures might be also possible in the nontranscribed strand. It was demonstrated that the nontranscribed strand is also subject to mutations during transcription that are not mediated by Mfd (Kim et al., 2010). In the case of the $\operatorname{gudB}$ mutation, this mechanism of mutagenesis is entirely conceivable. Possibly, further mechanisms of mutagenesis might contribute to the $g u d B$ mutation. In agreement with this hypothesis is that after the loss of the Mfd protein, the mutation rate is in fact hundred fold decreased but the acquisition of the mutation is not completely abolished and upon long term incubation of the $m f d$ rocG double mutant on complex medium $g u d B 1$ revertants still occur. Although the mutation rate of the mfd mutant is drastically reduced, a mutation rate in the range of $10^{-6}$ is still striking compared to other observed mutation rates that are in the range of $10^{-8}$ (Kunkel, 2004). This result implies that further factors are involved in the precise deletion of the $9 \mathrm{bp}$ in the direct repeat of the $\operatorname{gudB}$ gene. This topic has to be subject of further investigation. 


\subsection{The separation of enzymatic and regulatory activities of RocG}

In this work, two classes of mutant variants of the GDH RocG were isolated and characterized. Both classes uncouple the enzymatic and regulatory activities of this trigger enzyme RocG. Several single amino acid exchanges result in loss of enzymatic activity and are accompanied by permanent substrate-independent inactivation of GltC. This class of RocG variants was designated superrepressor RocG proteins (RocG-SR). A second class, exemplified by one mutant protein (designated monofunctional RocG, RocG-MF) has retained enzymatic activity but has lost the ability to inactivate GltC.

The mutations leading to the RocG SR phenotype are clustered in the active site of the enzyme. The separation of enzymatic and regulatory activities has already been observed for other trigger enzymes such as the B. subtilis glutamine synthetase (GS). The GS is regulated by glutamine feedback inhibition. The feedback inhibited GS triggers the DNA binding activity of two nitrogen transcriptional factors TnrA and GlnR (Wray et al., 2001; Fisher \& Wray, 2005). GS mutants that lost the capacity to regulate the activity of TnrA and GlnR also show mutations clustered to the region of the active site of the glutamine synthetase and are impaired in their enzymatic activity (Wray \& Fisher, 2005; Fisher \& Wray, 2009; Wray \& Fisher, 2010). Mutations causing the RocG superrepressor phenotype reduce the catalytic activity and the affinity for glutamate (see Tab. 2.3). Thus, it can be assumed that the conformation of these RocG variants required for GltC inhibition is more stable than that of the wild-type RocG enzyme. These variants might require less glutamate to elicit the "inhibitory" conformation of glutamate dehydrogenase. By contrast, the monofunctional RocG enzyme is enzymatically active, but glutamate does not induce the inhibitory conformation required to inactivate $\mathrm{GltC}$ activity.

Mutations affecting the control of gene expression by trigger enzymes have not only been found in the genes encoding the trigger enzymes. Similarly, mutations affecting the controlled transcription factors may interfere with their productive interaction with the cognate trigger enzyme. Such mutations have been isolated for GltC, and reduced interaction with RocG has been demonstrated for one of these variants (Belitsky \& Sonenshein, 1995; Commichau et al., 2007a).

An interesting result of this work is the identification of 10 different superrepressor variants of RocG, while only one monofunctional protein incapable of inhibiting GltC was found. The small number of monofunctional RocG variants isolated 
might indicate that the GltC interaction surface is relatively large and involves multiple side chains, and that the interaction between the two proteins and the inhibition of GltC by RocG cannot be easily disrupted by single amino acid substitutions in RocG.

The results presented support the idea of an inhibitory interaction between the glutamate dehydrogenase RocG and the transcription factor GltC. Moreover, they provide new insights into the relation between the enzymatic activities and the regulatory activities of the trigger enzyme RocG. The identification of the monofunctional RocG variant that has lost its regulatory function but has retained enzymatic activity is in excellent agreement with the previous conclusion: The enzymatic activity of RocG is important but not sufficient for the control exerted on GltC.

\subsection{A new model of RocG-GltC interaction}

An in vivo cross-linking approach revealed that the RocG-MF protein still interacts with the transcriptional regulator GltC, even though the inhibitory effect of this interaction on GltC is completely abolished. Thus, it can be assumed that the interaction itself is not sufficient to regulate GltC activity. Moreover, it is tempting to speculate that the mode of inhibition is based on a particular conformation of the RocG-GltC complex that might be excluded due to the mutation in the RocG-MF protein. The amino acid exchange of an aspartate to an asparagine at the position 122 is surface located. The surface exposed location of the mutated residue could have two implications. First, the aspartate residue could be essential for binding a yet unknown factor that mediates GltC regulation by RocG. Second, the aspartate residue 122 of the RocG protein is in the interface of the interaction and is essential to transmit an inhibitory signal to GltC. The hypothesis that a third protein or an unknown low molecular weight factor is involved in the regulation is supported by the failure to reconstruct the RocG-GltC interaction in vitro (our unpublished results). Other examples showed that the reconstructions of a particular regulatory protein-protein interaction can be a challenge in vitro. The concept of carbon catabolite repression (CCR) has been an intensively studied subject in E. coli. There is strong evidence that the phosphorylated subunit of the glucose permease exerts a regulatory role in the CCR by stimulating the activity of the adenylate cyclase (Görke 
\& Stülke, 2008). Nevertheless, this model has never been proven in vitro (Park et al., 2006).

In addition to the involvement of a third factor in the regulation of GltC by RocG it should be taken into account that the conformation of GltC might be important for regulation. GltC binds to different promoter sites in response to the presence of the low-molecular weight effectors, glutamate and 2-oxoglutarate (Picossi et al., 2007). High 2-oxoglutarate level favors the binding to the Boxes I and II in vitro which induces expression of the GOGAT. In the presence of glutamate GltC preferentially binds to the Boxes I and III leading to a loss of transcription activation. In vivo, this model is entirely conceivable to elucidate the regulation of GltC activity in the absence of RocG. But when RocG expression is induced, the RocG protein might be additionally responsible for sensing the glutamate to 2-oxoglutarate ratio and for transmitting this information to the GltC protein. This hypothesis might be explained with the following model (see Fig. 7.2). RocG interacts with GltC that has to bind previously to its target promoter region. It cannot be excluded that an additional protein is involved in the RocG-GltC interaction. In the model it is hypothesized that GltC has to be available in a particular quaternary structure that is formed upon DNA binding. Previous results suggest that GltC binds the DNA in a dimer-of-dimer fashion (Picossi et al., 2007). This assumption is supported by the observation that LysR regulators are functional as tetramer consisting of two dimers (Maddocks \& Oyston, 2008) If the glutamate level is high reflected by catalytic performance of RocG, the binding capacity of GltC might be altered leading to a lack of induction of the GOGAT expression (see Fig. 7.2A). The depletion of glutamate might facilitate a conformational change in the interaction that allows GltC activity (see Fig. 7.2B). This model hypothesizes that RocG triggers a switch in GltC activity dependent on the glutamate concentration in vivo. This idea is in agreement with the previous observations concerning the impact of RocG on GltC activity (Commichau et al., 2007a; 2007b). 

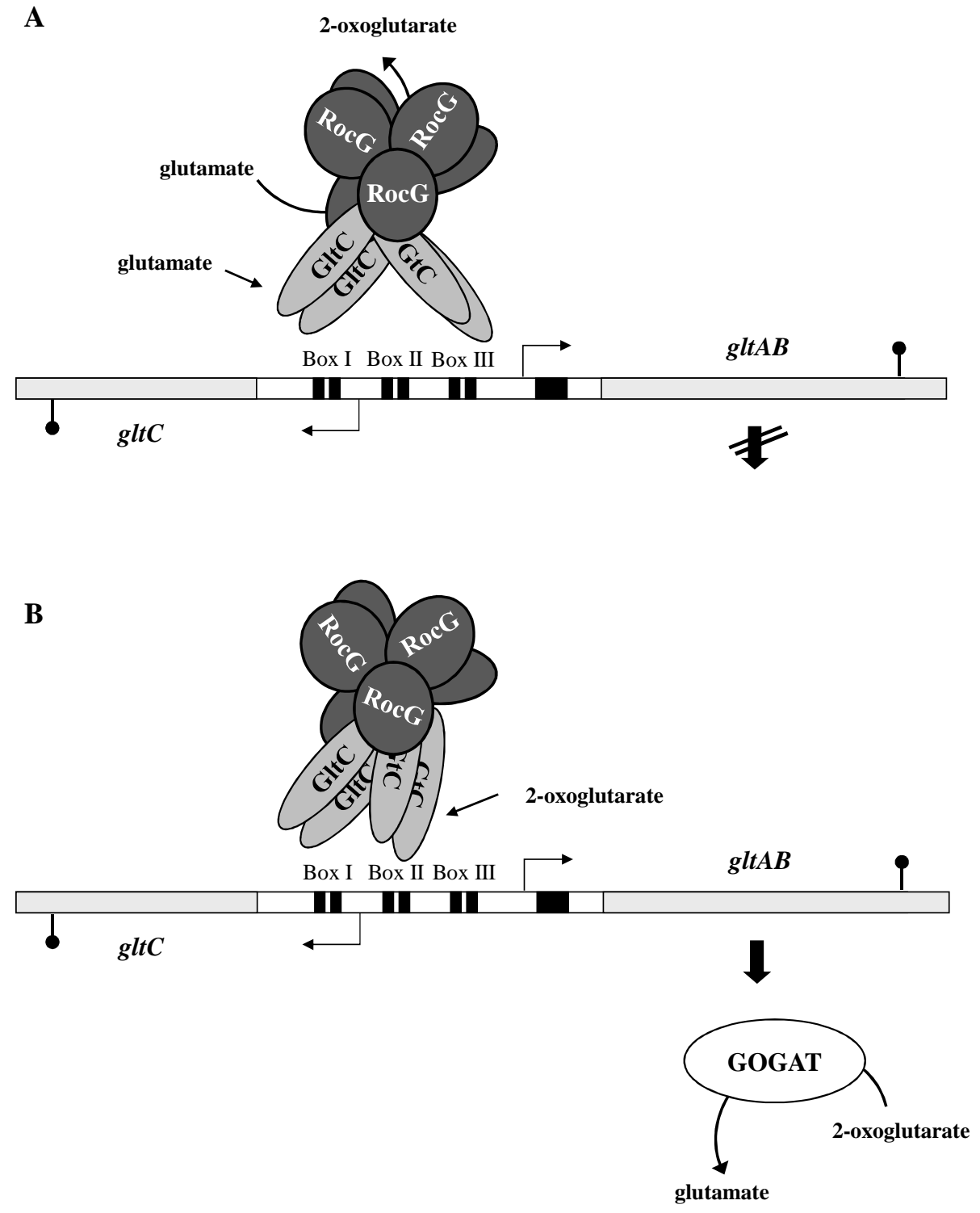

Fig. 7.2 - Model for the regulatory impact of RocG on GitC.

(A) RocG is capable of interacting with the transcriptional regulator GltC. In the presence of glutamate RocG carries out the degradation of glutamate to 2-oxoglutarate. The catalysis might favor a conformational change of GltC that does not allow the expression of the gltAB operon. (B) If the glutamate level drops, RocG might be no longer able to force GltC in an inhibitory conformation. This leads to the transcription of the gltAB operon. 
The model is also supported by the result that the RocG-SR proteins exert a strong inhibition on GltC in the presence of glutamate but do not influence GltC activity in the absence of glutamate. The activity in terms of glutamate degradation of the RocG-SR proteins is severely impaired and not sufficient to allow growth with arginine as sole carbon source. Nevertheless, they are still capable of catalyzing the reaction very slowly. This result leads to the hypothesis that the RocG-SR proteins are frozen in a glutamate bound state and therefore mediating a permanent strong inhibition of GltC activity. The RocG-MF protein is still capable of efficient glutamate utilization but lost the capacity to switch GltC activity. It is possible that RocG-MF cannot process an impact of an additional factor that might influence the effect of the RocG-GltC interaction. Another explanation might be that RocG-MF cannot transmit the information to GltC that leads to a conformational change of GltC multimers. These hypotheses have to be subject of further investigation. 


\subsection{Perspectives}

The results revealed the requirement of the Mfd protein in the rapid $\operatorname{gudB}$ decryptification. However, the DNA repair system that operates with Mfd in the mutagenesis has not been identified yet. In order to gain further insights into the Mfdmediated generation of gudBl mutations a SPINE experiment using a rocG mutant strain can be performed (Herzberg et al., 2007). By this in vivo cross linking approach potential interaction partners of the Mfd protein might be identified. To increase the chance of cross-linking Mfd in complex with its potential target the gudB gene and additional interaction partners, the $g u d B$ gene has to be enriched and transcribed at high level. This might be of importance as a high transcription rate is supposed to enhance the transcription coupled DNA repair mediated by Mfd (Gunka et al., subm.). Besides the analysis of the elution fraction by mass spectrometry to identify interaction partners of Mfd, a Northern blot with a $g u d B$ probe can be performed. This experiment might demonstrate whether the $g u d B$ transcript can be specifically cross-linked with the Mfd protein.

To test the hypothesis that the protein-protein interaction between RocG and GltC depends on the presence of the target promoter region of the transcription activator GltC, the defined DNA sequence can be introduced in the background of the bacterial two-hybrid assay. This can be simply achieved by cloning the promoter region into one of the vectors for the RocG and GltC co-expression. If the gltAB promoter is necessary to facilitate the right conformation of GltC and enables RocG to interact with GltC, this interaction might be detected in the bacterial two-hybrid assay in the presence of the promoter sequence.

A new SPINE approach might be of interest to identify the putative interaction partner that mediates the RocG-GltC interaction. With a newly developed mass spectrometry method the complete elution fractions of the co-purification can be analyzed very sensitively (E. Hammer, pers. comm.).

Furthermore, in vitro studies on the RocG-GltC interaction would be of great value to understand the regulatory mechanism. In order to check whether the RocGGltC interaction influences the binding properties of GltC to its target promoter region, DNA footprint analysis would be an appropriate approach. For this approach RocG-SR variants could be used in comparison to the RocG-MF protein, that was shown to lack GltC inhibition. 


\section{8. $\quad$ References}

Abramoff, M. D., Magelhaes, P. J., \& Ram, S. J. (2004) Image processing with ImageJ. Biophotonics. Internat. 11: 36-42.

Adams, P. D., Grosse-Kunstleve, R. W., Hung, L. W., Ioerger, T. R., McCoy, A., et al. (2002) PHENIX: building new software for automated crystallographic structure determination. Acta Crystallogr. D Biol. Crystallogr. 58: 1948-1954.

Ali, N. O., Jeusset, J., Larquet, E., Le Cam, E., Belitsky, B., et al. (2003) Specificity of the interaction of RocR with the rocG-rocA intergenic region in Bacillus subtilis. Microbiology 149: 739-750.

Arcondéguy, T., Jack, R. \& Merrick, M. (2001) P(II) signal transduction proteins, pivotal players in microbial nitrogen control. Microbiol. Mol. Biol. Rev. 65: 80-105.

Assenmacher, N. Wenig, K. Lammens, A. \& Hopfner, K. H. (2006) Structural basis for transcription-coupled repair: the $\mathrm{N}$ terminus of Mfd resembles UvrB with degenerate ATPase motifs. J. Mol. Biol. 355: 675-83.

Ayora, S., Rojo, F., Ogasawara, N., Nakai, S. \& Alonso. J. C. (1996) The Mfd protein of Bacillus subtilis 168 is involved in both transcription-coupled DNA repair and DNA recombination. J. Mol. Biol. 256: 301-318.

Bachem, S. \& Stülke, J. (1998) Regulation of the Bacillus subtilis GlcT antiterminator protein by components of the phosphotransferase system. J. Bacteriol. 180: 5319-5326.

Baker, N. A., Sept, D., Joseph, S., Holst, M. J. \& McCammon, J. A. (2001) Electrostatics of nanosystems: Application to microtubules and the ribosome. Proc. Natl Acad. Sci. U.S.A. 98: 10037-10041.

Baker, P. J., Waugh, M. L., Wang, X. G., Stillman, T. J., Turnbull, A. P., et al. (1997) Determinants of substrate specificity in the superfamily of amino acid dehydrogenases. Biochemistry 36: 16109-16115. 
Barbe, V., Cruveiller, S., Kunst, F., Lenoble, P., Meurice, G., et al. (2009) From a consortium sequence to a unified sequence: the Bacillus subtilis 168 reference genome a decade later. Microbiology 155: 1758-1775.

Barrick, J. E., Yu, D. S., Yoon, S. H., Jeong, H., Oh, T. K., et al. (2009) Genome evolution and adaptation in a long-term experiment with Escherichia coli. Nature 461: $1243-1247$.

Bayliss, C. D., Field, D. \& Moxon, E. R. (2001) The simple sequence contingency loci of Haemophilus influenzae and Neisseria meningitidis, J. Clin. Invest. 107: 657-662.

Beletskii, A., \& Bhagwat, A.S. (1996) Transcription-induced mutations: increase in C to $\mathrm{T}$ mutations in the nontranscribed strand during transcription in Escherichia coli. Proc. Natl. Acad. Sci. U. S. A. 93: 13919-13924.

Belitsky, B. R., Janssen, P. J. \& Sonenshein, A. L. (1995) Sites required for GltCdependent regulation of Bacillus subtilis glutamate synthase expression. J. Bacteriol. 177: 5686-5695.

Belitsky, B. R. \& Sonenshein, A. L. (1995) Mutations in GltC that increase Bacillus subtilis gltA expression. J. Bacteriol. 177: 5696-5700.

Belitsky, B. R. \& Sonenshein, A. L. (1997) Altered transcription activation specificity of a mutant form of Bacillus subtilis GltR, a LysR family member. J. Bacteriol. 179: 1035-1043.

Belitsky, B. R. \& Sonenshein, A. L. (1998) Role and regulation of Bacillus subtilis glutamate dehydrogenase genes. J. Bacteriol. 180: 6298-6305.

Belitsky, B. R. \& Sonenshein, A. L. (1999) An enhancer element located downstream of the major glutamate dehydrogenase gene of Bacillus subtilis. Proc. Natl. Acad. Sci. U.S.A. 96: 10290-10295.

Belitsky, B. R., Wray, L. V., Jr., Fisher, S. H., Bohannon, D. E. \& Sonenshein, A. L. (2000) Role of TnrA in nitrogen source-dependent repression of Bacillus subtilis glutamate synthase gene expression. J. Bacteriol. 182: 5939-5947. 
Belitsky, B. R., Kim, H. J. \& Sonenshein, A. L. (2004) CcpA-dependent regulation of Bacillus subtilis glutamate dehydrogenase gene expression. J. Bacteriol. 186: 3392 3398 .

Belitsky, B. R. \& Sonenshein, A. L. (2004) Modulation of activity of Bacillus subtilis regulatory proteins GltC and TnrA by glutamate dehydrogenase. J. Bacteriol. 186: 3399-3407.

Bennett, B. D., Kimball, E. H., Gao, M., Osterhout, R., Van Dien, S. J. \& Rabinowitz, J. D. (2009) Absolute metabolite concentrations and implied enzyme active site occupancy in Escherichia coli. Nat. Chem. Biol. 5: 593-599.

Bhuiya, M. W., Sakuraba, H., Ohshima, T., Imagawa, T., Katunuma, N. \& Tsuge, H. (2005) The first crystal structure of hyperthermostable NAD-dependent glutamate dehydrogenase from Pyrobaculum islandicum. J. Mol. Biol. 345: 325-337.

Bi, W. \& Stambrook, P. J. (1998) Site-directed mutagenesis by combined chain reaction. Anal. Biochem. 256: 137-140

Bichara, M., Wagner, J. \& Lambert, I. B. (2006) Mechanisms of tandem repeat instability in bacteria. Mutat. Res. 598: 144-163.

Blencke, H. M., Homuth, G., Ludwig, H., Mäder, U., Hecker, M. \& Stülke, J. (2003) Transcriptional profiling of gene expression in response to glucose in Bacillus subtilis: regulation of the central metabolic pathways. Metab. Eng. 5: 133-149.

Bohannon, D. E., Rosenkrantz, M. S. \& Sonenshein, A. L. (1985) Regulation of Bacillus subtilis glutamate synthase genes by the nitrogen source. J. Bacteriol. 163: 957-964.

Bohannon, D. E. \& Sonenshein, A. L. (1989) Positive regulation of glutamate biosynthesis in Bacillus subtilis. J. Bacteriol. 171: 4718-4727.

Bohr, V. A., Smith, C. A., Okumoto, D. S. \& Hanawalt, P. C. (1985) DNA repair in an active gene: removal of pyrimidine dimers from the DHFR gene of $\mathrm{CHO}$ cells is much more efficient than in the genome overall. Cell 40: 359-369. 
Boylan, S. A., Redfield, A. R., Brody, M. S. \& Price, C. W. (1993) Stress-induced activation of the sigma B transcription factor of Bacillus subtilis. J. Bacteriol. 175: 7931-7937.

Branda, S. S., Gonzalez-Pastor, J. E., Ben-Yehuda, S., Losick, R. \& Kolter, R. (2001) Fruiting body formation by Bacillus subtilis. Proc. Natl Acad. Sci. U.S.A. 98: 11621-11626.

Branda, S. S., González-Pastor, J. E., Dervyn, E., Ehrlich, S. D., Losick, R. \& Kolter R. (2004) Genes involved in formation of structured multicellular communities by Bacillus subtilis. J. Bacteriol. 186: 3970-3979.

Braun, P., Tasan, M., Dreze, M., Barrios-Rodiles, M., Lemmens, I., et al. (2009) An experimentally derived confidence score for binary protein-protein interactions. Nat. Methods 6: $91-97$.

Britton, K. L., Yip, K. S., Sedelnikova, S. E., Stillman, T. J., Adams, et al. (1999) Structure determination of the glutamate dehydrogenase from the hyperthermophile Thermococcus litoralis and its comparison with that from Pyrococcus furiosus. J. Mol. Biol. 293: 1121-1132.

Borukhov, S., Lee, J. \& Laptenko, O. (2005) Bacterial transcription elogantion factors: new insights into molecular mechanism of action. Mol. Microbiol. 55: 13151342.

Cairns, J. \& Foster, P. L. (1991) Adaptive reversion of a frameshift mutation in Escherichia coli. Genetics 128: 695-701.

Cairns, J., Overbaugh, J. \& Miller, S. (1988) The origin of mutants. Nature 335: 142145 .

Calogero, S., Gardan, R., Glaser, P., Schweizer, J., Rapoport, G. \& Débarbouillé, M. (1994) RocR, a novel regulatory protein controlling arginine utilization in Bacillus subtilis, belongs to the NtrC/NifA family of transcriptional activators. J. Bacteriol. 176: 1234-1241. 
CCP4 (1994) The CCP4 suite: programs for protein crystallography. Acta Crystallogr. D Biol. Crystallogr. 50: 760-763.

Chagneau, C. \& Saier, M. H., Jr. (2004) Biofilm-defective mutants of Bacillus subtilis. J. Mol. Microbiol. Biotechnol. 8: 177-188.

Chédin, F., Noirot, P., Biaudet, V., \& Ehrlich, S. D. (1998) A five-nucleotide sequence protects DNA from exonucleolytic degradation by AddAB, the RecBCD analogue of Bacillus subtilis. Mol. Microbiol. 29: 1369-1377.

Chin, G., Jr., Chavarria, D. G., Nakamura, G. C. \& Sofia, H. J. (2008) BioGraphE: high-performance bionetwork analysis using the Biological Graph Environment. BMC Bioinformatics 9: S6.

Chumsakul, O., Takahashi, H., Oshima, T., Hishimoto, T., Kanaya, S., et al. (2010) Genome-wide binding profiles of the Bacillus subtilis transition state regulator AbrB and its homolog Abh reveals their interactive role in transcriptional regulation. Nucleic Acids Res. in press (PMID: 20817675).

Claessen, D., Emmins, R., Hamoen, L. W., Daniel, R. A., Errington, J. \& Edwards, D. H. (2008) Control of the cell elongation-division cycle by shuttling of PBP1 protein in Bacillus subtilis. Mol. Microbiol. 68: 1029-1046.

Claessen, K., Een, N., Sheeran, M., Sörensson, N., Voronov, A. \& Åkesson, K. (2009) SAT-solving in practice, with a tutorial example from supervisory control. Discrete Event Dyn. Syst. 19: 495-524.

Claverys, J. P. \& Håvarstein, L. S. (2007) Cannibalism and fratricide: mechanisms and raisons d'être. Nat. Rev. Microbiol. 5: 219-229.

Commichau, F. M. (2006) Regulation der Glutamatsynthese in Bacillus subtilis durch die Glutamatdehydrogenase RocG und das Aktivatorprotein GltC. PhD thesis. GeorgAugust-Universität Göttingen; Germany

Commichau, F. M., Forchhammer, K. \& Stülke J. (2006) Regulatory links between carbon and nitrogen metabolism. Curr. Opin. Microbiol. 9: 167-172. 
Commichau, F. M., Herzberg, C., Tripal, P., Valerius, O. \& Stülke, J. (2007a) A regulatory protein-protein interaction governs glutamate biosynthesis in Bacillus subtilis: the glutamate dehydrogenase RocG moonlights in controlling the transcription factor GltC. Mol. Microbiol. 65: 642-654.

Commichau, F. M., Wacker, I., Schleider, J., Blencke, H. M., Reif, I., et al. (2007b) Characterization of Bacillus subtilis mutants with carbon-source independent glutamate biosynthesis. J. Mol. Microbiol. Biotechnol. 12: 106-113.

Commichau, F M., Gunka, K., Landmann, J. J. \& Stülke, J. (2008) Glutamate metabolism in Bacillus subtilis: gene expression and enzyme activities evolved to avoid futile cycles and to allow rapid responses to perturbations of the system. J. Bacteriol. 190: $3557-3564$.

Commichau, F. M. \& Stülke, J. (2008) Trigger enzymes: bifunctional proteins active in metabolism and in controlling gene expression. Mol. Microbiol. 67: 692-702.

da Costa, M. S., Santos, H. \& Galinski, E. A. (1998) An overview of the role and diversity of compatible solutes in Bacteria and Archaea. Adv. Biochem. Eng. Biotechnol. 61: 117-153.

Darwin, C. R. (1859) The origin of species. Ed. Avenel Books, New York 1979.

de Figueiredo, L. F., Podhorski, A., Rubio, A., Kaleta, C., Beasley, J. E., et al. (2009) Computing the shortest elementary flux modes in genome-scale metabolic networks. Bioinformatics 25: 3158-3165.

de Jong, H. \& Page, M. (2008) Search for steady states of piecewise-linear differential equation models of genetic regulatory networks. IEEE/ACM Trans Comput Biol Bioinform 5: 208-22.

Deaconescu, A. M., Chambers, A. L., Smith, A. J., Nickels, B. E., Hochschild, A., et al. (2006) Structural basis for bacterial transcription-coupled DNA repair. Cell 124: 507-520. 
Débarbouillé, M., Martin-Verstraete, I., Kunst, F. \& Rapoport, G. (1991) The Bacillus subtilis sigL gene encodes an equivalent of sigma 54 from gram-negative bacteria. Proc. Natl .Acad. Sci. U.S.A. 88: 9092-9096.

Denamur, E. \& Matic, I. (2006) Evolution of mutation rates in bacteria. Mol. Microbiol. 60: 820-7.

Deshpande, K. L. \& Kane, J. F. (1980) Glutamate synthase from Bacillus subtilis: in vitro reconstitution of an active amidotransferase. Biochem. Biophys. Res. Commun. 93: $308-314$.

Detsch, C. \& Stülke, J. (2003) Ammonium utilization in Bacillus subtilis: transport and regulatory functions of $\mathrm{NrgA}$ and $\mathrm{NrgB}$. Microbiology 149: 3289-3297.

Deuel, T. F. \& Prusiner, S. (1974) Regulation of glutamine synthetase from Bacillus subtilis by divalent cations, feedback inhibitors, and L-glutamine. J. Biol. Chem. 249: 257-264.

Eisen, J. A. \& Hanawalt, P. C. (1999) A phylogenomic study of DNA repair genes, proteins, and processes. Mutat. Res. 43: 171-213.

Emsley, P. \& Cowtan, K. (2004) Coot: model-building tools for molecular graphics. Acta Crystallogr. D Biol. Crystallogr. 60: 2126-2132.

Evans, P. R. (1993) Data collection and processing. Proc. CCP4 Study Weekend, 114122.

Feavers, I. M., Price, V. \& Moir, A. (1998) The regulation of the fumarase (citG) gene of Bacillus subtilis 168. Mol. Gen. Genet. 211: 465-471.

Feist, A. M. \& Palsson, B. Ø. (2008) The growing scope of applications of genomescale metabolic reconstructions using Escherichia coli. Nat. Biotechnol. 26: 659-667.

Fisher, S. H. (1999) Regulation of nitrogen metabolism in Bacillus subtilis: vive la différence! Mol. Microbiol. 32: 223-232. 
Fisher, S. H. \& Débarbouillé, M. (2002) Nitrogen Source Utilization and Its Regulation. A. L. Sonenshein, J. A. Hoch and R. Losick. Bacillus subtilis and Its Closest Realtives:from Genes to Cells. ASM Press, Washington, D. C. pp. 181-191.

Fisher, S. H. \& Wray, L. V., Jr. (2002) Bacillus subtilis 168 contains two differentially regulated genes encoding L-asparaginase. J. Bacteriol. 184: 2148-54.

Fisher, S. H. \& Wray, L. V., Jr. (2008) Bacillus subtilis glutamine synthetase regulates its own synthesis by acting as a chaperone to stabilize GlnR-DNA complexes. Proc. Natl. Acad. Sci. U.S.A. 105: 1014-1019.

Fisher, S. H. \& Wray, L. V., Jr. (2009) Novel transacting Bacillus subtilis glnA mutations that derepress $g \ln R A$ expression. J. Bacteriol. 191: 2485-2492.

Flórez, L. A., Roppel, S. F., Schmeisky, A. G., Lammers, C. R. \& Stülke J. (2009) A community-curated consensual annotation that is continuously updated: the Bacillus subtilis centred wiki SubtiWiki. Database 2009:bap012.

Flórez, L. A., Gunka, K., Polanía, R., Tholen, S. \& Stülke J. SPABBATS: A pathway-discovery method based on Boolean satisfiability that facilitates the characterization of suppressor mutants BMC Systems Biology submitted

Fousteri, M. \& Mullenders, L. F. H. (2008) Transcription-coupled nucleotide excision repair in mammalian cells: molecular mechanisms and biological effects. Cell Res. 8: 73-84.

Fränzle, M., Herde, C. \& Teige, T. (2007) Efficient solving of large non-linear arithmetic constraint systems with complex Boolean structure. Journal on Satisfiability 1: 209-236.

Frimmersdorf, E., Horatzek, S., Pelnikevich, A., Wiehlmann, L. \& Schomburg, D. (2010) How Pseudomonas aeruginosa adapts to various environments: a metabolomic approach. Environ. Microbiol. 12: 1734-1747.

Fukui, K. (2010) DNA mismatch repair in eukaryotes and bacteria. J. Nucleic. Acids. pii: 260512 . 
Gardan, R., Rapoport, G. \& Débarbouillé, M. (1995) Expression of the rocDEF operon involved in arginine catabolism in Bacillus subtilis. J. Mol .Biol. 249: 843-856.

Gardan, R., Rapoport, G. \& Débarbouillé, M. (1997) Role of the transcriptional activator RocR in the arginine-degradation pathway of Bacillus subtilis. Mol. Microbiol. 24: $825-837$.

Gardner, A. L. \& Aronson, A. I. (1984) Expression of the Bacillus subtilis glutamine synthetase gene in Escherichia coli. J. Bacteriol. 158: 967-971.

Gerth, U., Kock, H., Küsters, I., Michalik, S., Switzer, R. L. \& Hecker, M. (2008) Clp-dependent proteolysis down-regulates central metabolic pathways in glucosestarved Bacillus subtilis. J. Bacteriol. 190: 321-331.

Goelzer, A., Bekkal Brikci, F., Martin-Verstraete, I., Noirot, P., Bessières, P., et al. (2008) Reconstruction and analysis of the genetic and metabolic regulatory networks of the central metabolism of Bacillus subtilis. BMC Syst Biol 2: 20.

Gordon, R. E. (1981) One hundred and seven years of the genus Bacillus. R. C. Berkeley, and M. Goodfellow (ed.) The aerobic endosporeforming bacteria. Published for the Society for General Microbiology by Academic Press, London ; New York.

Görke, B. \& Stülke, J. (2008) Carbon catabolite repression in bacteria: many ways to make the most out of nutrients. Nat. Rev. Microbiol. 6: 613-624.

Goss, T. J., Perez-Matos, A. \& Bender, R. A. (2001) Roles of glutamate synthase, gltBD, and gltF in nitrogen metabolism of Escherichia coli and Klebsiella aerogenes. $\mathrm{J}$ Bacteriol. 183: 6607-6619.

Graça, A., Marques-Silva, J., Lyncem I. \& Oliveira, A. L. (2007) Efficient haplotype inference with pseudo-Boolean optimization. In Algebraic Biology Springer Verlag Berlin/Heidelberg: 2007:125-139.

Greener, A. \& Callahan, M. (1994) XL1-Red: a highly efficient random mutagenesis strain. Strategies 7: 32-34. 
Guérout-Fleury, A. M., Shazand, K., Frandsen, N. \& Stragier, P. (1995) Antibiotic resistance cassettes for Bacillus subtilis. Gene 167: 335-336.

Gunasekaran, K. \& Nussinov, R. (2007) How different are structurally flexible and rigid binding sites? Sequence and structural features discriminating proteins that do and do not undergo conformational change upon ligand binding. J. Mol. Biol. 365: 257-273

Gunka, K., Newman, J. A., Commichau, F. M., Herzberg, C., Rodrigues, C., et al. (2010) Functional dissection of a trigger enzyme: mutations of the Bacillus subtilis glutamate dehydrogenase RocG that affect differentially its catalytic activity and regulatory properties. J. Mol. Biol. 400: 815-827.

Gunka, K., Tholen, S., Neme, R., Freytag, B., Commichau, F. M., et al. A highfrequency mutation in Bacillus subtilis: Requirements for the decryptification of the gudB glutamate dehydrogenase gene. Mol. Microbiol. submitted

Guo, C., Tang, T. S. \& Friedberg, E. C. (2010) SnapShot: nucleotide excision repair. Cell 140: 754-4.e1.

Hackett, R. H. \& Setlow, P. (1988) Properties of spores of Bacillus subtilis strain which lack the major small, acid-soluble protein. J. Bacteriol. 170: 1403-1404

Hall, B. G. \& Betts, P. W. (1987) Cryptic genes for cellobiose utilization in natural isolates of Escherichia coli, Genetics 115: 431-439.

Hamoen, L. W., Venema, G. \& Kuipers, O. P. (2003) Controlling competence in Bacillus subtilis: shared use of regulators. Microbiology 149: 9-17.

Hanawalt, P. C. \& Spivak, G. (2008) Transcription-coupled DNA repair: two decades of progress and surprises Nat. Rev. Mol. Cell Biol. 9: 958-970.

Harwood, C. (1990) Molecular biological methods for Bacillus. Cutting, S. M. (ed.) John Wiley \& Sons, Ltd., Chichester, United Kingdom.

Hecker, M., Pané-Farré, J. \& Völker, U. (2007) SigB-dependent general stress response in Bacillus subtilis and related Gram-positive bacteria. Annu. Rev. Microbiol. 61: $215-236$. 
Helmann, J. D., Wu, M. F., Kobel, P. A., Gamo, F. J., Wilson, M., et al. (2001) Global transcriptional response of Bacillus subtilis to heat shock. J. Bacteriol. 183: 7318-7328.

Herzberg, C., Weidinger, L. A., Dörrbecker, B., Hübner, S., Stülke, J. \& Commichau, F. M. (2007) SPINE: a method for the rapid detection and analysis of protein-protein interactions in vivo. Proteomics 7: 4032-4035.

Ikeda, T. P., Shauger, A. E. \& Kustu, S. (1996) Salmonella typhimurium apparently perceives external nitrogen limitation as internal glutamine limitation. J. Mol. Biol. 259: 589-607.

Imhof, M. \& Schlötterer, C. (2001) Fitness effects of advantageous mutations in evolving Escherichia coli populations. Proc. Natl. Acad. Sci. U.S.A. 98: 1113-1117.

Jeffery, C. J. (1999). Moonlighting proteins. Trends Biochem. Sci. 24: 8-11.

Jin, S. \& Sonenshein, A. L. (1994): Transcriptional regulation of Bacillus subtilis citrate synthase genes. J. Bacteriol. 176: 4680-4690.

Jinap, S. \& Hajeb, P. (2010) Glutamate. Its applications in food and contribution to health. Appetite 55: 1-10.

Karimova, G., Pidoux, J., Ullmann, A. \& Ladant, D., (1998) A bacterial two-hybrid system based on a reconstituted signal transduction pathway. Proc. Natl. Acad. Sci. U.S.A. 95: 5752-5756.

Kearns, D. B. \& Losick, R. (2003) Swarming motility in undomesticated Bacillus subtilis. Mol. Microbiol. 49: 581-590.

Kempf, B. \& Bremer, E. (1998) Uptake and synthesis of compatible solutes as microbial stress responses to high-osmolality environments. Arch. Microbiol. 170: 319330.

Kim, H., Lee, B. S., Tomita, M. \& Kanai, A. (2010). Transcription-associated mutagenesis increases protein sequence diversity more effectively than does random mutagenesis in Escherichia coli. PLoS ONE 5: e10567. 
Kim, N., Abdulovic, A. L., Gealy, R., Lippert, M. J. \& Jinks-Robertson, S. (2007) Transcription-associated mutagenesis in yeast is directly proportional to the level of gene expression and influenced by the direction of DNA replication. DNA Repair 6: 1285-1296.

Kimura, K., Tran, L.-S. P. \& Itoh, Y. (2004) Roles and regulation of the glutamate racemase isogenes, racE and yrpC, in Bacillus subtilis. Microbiology 150: 2911-2920.

Klamt, S. \& Stelling, J. (2002) Combinatorial complexity of pathway analysis in metabolic networks. Mol. Biol. Rep. 29: 233-236.

Klapacz, J. \& Bhagwat, A. S. (2005) Transcription promotes guanine to thymine mutations in the non-transcribed strand of an Escherichia coli gene. DNA Repair 4: 806-813.

Klingel, U., Miller, C. M., North, A. K., Stockley, P. G. \& Baumberg, S. (1995) A binding site for activation by the Bacillus subtilis $\mathrm{AhrC}$ protein, a repressor/activator of arginine metabolism. Mol. Gen. Genet. 248: 329-340.

Knapp, S., de Vos, W. M., Rice, D. W. \& Ladenstein, R. (1997) Crystal structure of glutamate dehydrogenase from the hyperthermophilic eubacterium Thermotoga maritima at 3.0 Å resolution. J. Mol. Biol. 267: 916-932.

Kondoh, T. \& Torii, K. (2008) Brain activation by Umami substances via gustatory and visceral signaling pathways, and physiological significance. Biol. Pharm. Bull. 31: 1827-1832.

Kunkel, T. A. (2004) DNA replication fidelity. J. Biol. Chem. 279: 16895-16898.

Kunst, F. \& Rapoport, G. (1995) Salt stress is an environmental signal affecting degradative enzyme synthesis in Bacillus subtilis. J. Bacteriol. 177: 2403-2407.

Kunst, F., Ogasawara, N., Moszer, I., Albertini, A. M., Alloni, G., et al. (1997) The complete genome sequence of the Gram-positive bacterium Bacillus subtilis. Nature 390: $249-256$. 
Lammers, C. R., Flórez, L. A., Schmeisky, A. G., Roppel, S. F., Mäder, U., et al. (2010) Connecting parts with processes: SubtiWiki and SubtiPathways integrate gene and pathway annotation for Bacillus subtilis. Microbiology 156: 849-859.

Laskowski, R. A., MacAurthur, M. W., Moss, D. S. \& Thornton, J. M. (1993). PROCHECK: a program to check the stereochemical quality of protein structures. J. Appl. Crystallogr. 26: 283-291.

Lea, D. E., \& Coulson, C. A. (1949) The distribution of mutants in bacterial populations. J. Genet. 49: 248-264.

Leigh, J. A. \& Dodsworth, J. A. (2007) Nitrogen regulation in Bacteria and Archaea. Annu. Rev. Microbiol. 61: 349-377.

Lemon, K. P., Kurtser, I. \& Grossman, A. D. (2001) Effects of replication termination mutants on chromosome partitioning in Bacillus subtilis. Proc. Natl. Acad. Sci. U.S.A. 98: 212-217.

Lehnik-Habrink, M., Pförtner, H., Rempeters, L., Pietack, N., Herzberg, C. \& Stülke, J. (2010) The RNA degradosome in Bacillus subtilis: identification of CshA as the major RNA helicase in the multiprotein complex. Mol. Microbiol. 77: 958-971.

Leslie, A. G. W. (1992) Joint CCP4 + ESF-EAMCB. Newsl. Protein Crystallogr. 26.

López, D., Vlamakis, H. \& Kolter, R. (2010) Biofilms. Cold Spring Harb Perspect Biol 2: a000398.

Lou, C., Liu, X., Ni, M., Huang, Y., Huang, Q., et al. (2010) Synthesizing a novel genetic sequential logic circuit: a push-on push-off switch. Mol. Syst. Biol. 6: 350.

Ludwig, H., Homuth, G., Schmalisch, M., Dyka, F. M., Hecker M. \& Stülke, J. (2001) Transcription of glycolytic genes and operons in Bacillus subtilis: Evidence for the presence of multiple levels of control of the gapA operon. Mol. Microbiol. 41: 409422.

Luria, S. E. \& Delbrück, M. (1943) Mutations of bacteria from virus sensitivity to virus resistance. Genetics 28: 491-511. 
Maddocks, S. E. \& Oyston, P. C. (2008) Structure and function of the LysR-type transcriptional regulator (LTTR) family proteins. Microbiology 154: 3609-3623.

Magasanik, B. (2003) Ammonia assimilation by Saccharomyces cerevisiae. Eukaryotic Cell 2: 827-829.

Martin, P., Makepeace, K., Hill, S. A., Hood, D. W. \& Moxon, E. R. (2005) Microsatellite instability regulates transcription factor binding and gene expression. Proc. Natl. Acad. Sci. U.S.A 102: 3800-3804.

Martin-Verstraete, I., Débarbouillé, M., Klier, A. \& Rapoport, G. (1992) Mutagenesis of the Bacillus subtilis "-12,-24" promoter of the levanase operon and evidence for the existence of an upstream activating sequence. J. Mol. Biol. 226: 85-99.

Martin-Verstraete, I., Débarbouillé, M., Klier, A. \& Rapoport, G. (1994) Interactions of wild-type and truncated LevR of Bacillus subtilis with the upstream activating sequence of the levanase operon. J. Mol. Biol. 241: 178-192.

Martin-Verstraete, I., Charrier, V., Stülke, J., Galinier, A., Erni, B., et al. (1998) Antagonistic effects of dual PTS catalyzed phosphorylation on the Bacillus subtilis transcriptional activator LevR. Mol. Microbiol. 28: 293-303.

Matsuoka, K. \& Kimura, K. (1986) Glutamate synthase from Bacillus subtilis PCI 219. J. Biochem. (Tokyo) 99: 1087-1100.

Meinken, C., Blencke, H. M., Ludwig, H. \& Stülke, J. (2003) Expression of the glycolytic gapA operon in Bacillus subtilis: differential syntheses of proteins encoded by the operon. Microbiology 149: 751-761.

Mellon, I. \& Champe, G. N. (1996) Products of DNA mismatch repair genes mutS and mutL are required for transcription-coupled nucleotide-excision repair of the lactose operon in Escherichia coli. Proc. Natl. Acad. Sci. U.S.A. 93: 1292-1297.

Merzbacher, M., Detsch, C., Hillen, W. \& Stülke, J. (2004) Mycoplasma pneumoniae HPr kinase/phosphorylase: assigning functional roles to the P-loop and the HPrK/P signature sequence motif. Eur. J. Biochem. 271: 367-374. 
Meyer, F. M., Gerwig, J., Hammer, E., Herzberg, C., Commichau, F. M., et al. (2011) Physical interactions between tricarboxylic acid cycle enzymes in Bacillus subtilis: evidence for a metabolon. Metab, Engn. in press (PMID: 20933603).

Michel, B. (2000) Replication fork arrest and DNA recombination. Trends Biochem. Sci. 25: 173-178.

Miller, C. M., Baumberg, S. \& Stockley, P. G. (1997) Operator interactions by the Bacillus subtilis arginine repressor/activator, AhrC: novel positioning and DNAmediated assembly of a transcriptional activator at catabolic sites. Mol. Microbiol. 26: $37-48$.

Modrich, P. \& Lahue, R. (1996) Mismatch repair in replication fidelity, genetic recombination, and cancer biology. Annu. Rev. Biochem. 65: 101-133.

Morett, E. \& Segovia, L. (1993) The sigma 54 bacterial enhancer-binding protein family: mechanism of action and phylogenetic relationship of their functional domains. J. Bacteriol. 175: 6067-6074.

Muro-Pastor, A. M. \& Maloy, S. (1995) Proline dehydrogenase activity of the transcriptional repressor PutA is required for induction of the put operon by proline. $\mathrm{J}$. Biol. Chem. 270: 9819-9827.

Murshudov, G. N., Vagin, A. A. \& Dodson, E. J. (1997) Refinement of macromolecular structures by the maximum-likelihood method. Acta Crystallogr. D Biol. Crystallogr. 53: 240-255.

Nakasako, M., Fujisawa, T., Adachi, S., Kudo, T. \& Higuchi, S. (2001) Large-scale domain movements and hydration structure changes in the active-site cleft of unligated glutamate dehydrogenase from Thermococcus profundus studied by cryogenic X-ray crystal structure analysis and small-angle X-ray scattering. Biochemistry 40: 30693079 .

Ninfa, A. J. \& Jiang, P. (2005) PII signal transduction proteins: sensors of alphaketoglutarate that regulate nitrogen metabolism. Curr. Opin. Microbiol. 8: 168-173. 
Oh, Y. K., Palsson, B. Ø., Park, S. M., Schilling , C. H. \& Mahadevan, R. (2007) Genome-scale reconstruction of metabolic network in Bacillus subtilis based on highthroughput phenotyping and gene essentiality data. J. Biol. Chem. 282: 28791-28799.

Orth, J. D., Thiele, I. \& Palsson, B. Ø. (2010) What is flux balance analysis? Nat. Biotechnol. 28: 245-248.

Park, Y. H., Lee, B. R., Seok, Y.J. \& Peterkofsky, A. (2006) In vitro reconstitution of catabolite repression in Escherichia coli. J Biol Chem. 281:6448-6454.

Patrick, J. E. \& Kearns, D. B. (2009) Laboratory strains of Bacillus subtilis do not exhibit swarming motility. J. Bacteriol. 191: 7129-7133.

Persuh, M., Mandic-Mulec, I. \& Dubnau, D. (2002) A MecA paralog, YpbH, binds ClpC, affecting both competence and sporulation. J Bacteriol. 184: 2310-2313.

Picossi, S., Belitsky, B. R. \& Sonenshein, A. L. (2007) Molecular mechanism of the regulation of Bacillus subtilis gltAB expression by GltC. J. Mol. Biol. 365: 1298-1313.

Piran, R., Halperin, E., Guttmann-Raviv, N., Keinan, E. \& Reshef, R. (2009) Algorithm of myogenic differentiation in higher-order organisms. Development 136: 3831-3840.

Planes, F. J. \& Beasley, J. E. (2008) A critical examination of stoichiometric and pathfinding approaches to metabolic pathways. Brief. Bioinformatics 9: 422-436.

Polosina, Y. Y. \& Cupples, C. G. (2010) MutL: conducting the cell's response to mismatched and misaligned DNA. Bioessays 32: 51-59.

Pomerantz, R. T., O'Donnell, M. (2010) Direct restart of a replication fork stalled by a head-on RNA polymerase. Science 327: 590-592.

Pybus, C., Pedraza-Reyes, M., Ross, C. A., Martin, H., Ona, K., et al. (2010) Transcription-associated mutation in Bacillus subtilis cells under stress. J. Bacteriol. 192: $3321-3328$. 
Ramachandran, G. N., Ramakrishnan, C. \& Sasisekharan, V. (1963). Stereochemistry of polypeptide chain configurations. J. Mol. Biol. 7: 95-99.

Rehm, N. \& Burkovski A. (2010) Engineering of nitrogen metabolism and its regulation in Corynebacterium glutamicum: influence on amino acid pools and production. Appl. Microbiol. Biotechnol. in press (PMID: 20922371).

Reitzer, L. J. (1996) Ammonia assimilation and the biosynthesis of glutamine, glutamate, aspartate, asparagine, L-alanine, and D-alanine, p. 391-407. In F. C. Neidhardt, R. Curtiss III, J. L. Ingraham, E. C. C. Lin, K. B. Low, B. Magasanik, W. S. Reznikoff, M. Riley, M. Schaechter, and H. E. Umbarger (ed.), Escherichia coli and Salmonella: cellular and molecular biology, 2nd ed. American Society for Microbiology, Washington, D.C.

Reitzer, L. (2003) Nitrogen assimilation and global regulation in Escherichia coli. Annu. Rev. Microbiol. 57: 155-176.

Rietkötter, E., Hoyer, D. \& Mascher, T. (2008) Bacitracin sensing in Bacillus subtilis. Mol. Microbiol. 68: 768-785.

Roberts, J. \& Park J. S. (2004) Mfd, the bacterial repair coupling factor: translocation, repair and termination. Curr. Opin. Microbiol. 7: 120-125

Robleto, E. A., Yasbin, R., Ross, C. \& Pedraza-Reyes, M. (2007) Stationary phase mutagenesis in B. subtilis: a paradigm to study genetic diversity programs in cells under stress. Crit. Rev. Biochem. Mol. Biol. 42: 327-339

Ross. C., Pybus, C., Pedraza-Reyes, M., Sung, H. M., Yasbin, R. E. \& Robleto, E. (2006) Novel role of mfd: effects on stationary-phase mutagenesis in Bacillus subtilis. J. Bacteriol. 188: 7512-7520.

Rossmann, M. G., Moras, D. \& Olsen, K. W. (1974) Chemical and biological evolution of nucleotide-binding protein. Nature 250: 194-199.

Rossolillo, P. \& Albertini, A. M. (2001) Functional analysis of the Bacillus subtilis yshD gene, a mutS paralogue. Mol. Gen. Genet. 264: 809-818. 
Roth, J. R., Kugelberg, E., Reams, A. B., Kofoid, E. \& Andersson, D. I. (2006) Origin of mutations under selection: the adaptive mutation controversy. Annu. Rev. Microbiol. 60: 477-501.

Ruzal, S. M. \& Sanchez-Rivas, C. (2003) Effect of glutamate synthase (GOGAT) activity on Bacillus subtilis spore properties. Curr. Microbiol. 47: 208-213.

Sambrook, J., Fritsch, E. F. \& Maniatis, T. (1989) Molecular cloning: a laboratory manual, 2nd edit. Cold Spring Harbor Laboratory, Cold Spring Harbor, NY.

Sancar, A. (1994) Mechanisms of DNA excision repair. Science 266: 1954-1956.

Sano, C. (2009) History of glutamate production. Am. J. Clin. Nutr. 90: 728S-732S

Schilling, O., Frick, O., Herzberg, C., Ehrenreich, A., Heinzle, E., et al. (2007) Transcriptional and metabolic responses of Bacillus subtilis to the availability of organic acids: transcription regulation is important but not sufficient to account for metabolic adaptation. Appl. Environ. Microbiol. 73: 499-507.

Schirmer, F., Ehrt, S. \& Hillen, W (1997) Expression, inducer spectrum domain structure, and function of MopR, the regulator of phenol degradation in Acinetobacter calcoaceticus NCIB8250. J. Bacteriol. 179: 1329-1336.

Schmalisch, M. H., Bachem, S. \& Stülke, J. (2003) Control of the Bacillus subtilis antiterminator protein GlcT by phosphorylation: Elucidation of the phosphorylation chain leading to inactivation of GlcT. J. Biol. Chem. 278: 51108-51115.

Schreier, H. J., Brown, S. W., Hirschi, K. D., Nomellini, J. F. \& Sonenshein, A. L. (1989) Regulation of Bacillus subtilis glutamine synthetase gene expression by the product of the $g \ln R$ gene. J. Mol. Biol. 210: 51-63.

Selby, C. P. \& Sancar, A. (1993) Molecular mechanism of transcription-repair coupling. Science 260: 53-58

Selby, C. P. \& Sancar, A. (1994) Mechanisms of transcription-repair coupling and mutation frequency decline. Microbiol. Rev. 58: 317-329. 
Selby, C. P. \& Sancar, A. (1995a) Structure and function of transcription-repair coupling factor. I. Structural domains and binding properties. J. Biol. Chem. 270: 48824889.

Selby, C. P. \& Sancar, A. (1995b) Structure and function of transcription-repair coupling factor. II. Catalytic properties. J. Biol. Chem. 270: 4890-4895.

Sharkey, M. A. \& Engel, P. C. (2008) Apparent negative co-operativity and substrate inhibition in overexpressed glutamate dehydrogenase from Escherichia coli. FEMS Microbiol. Lett. 281: 132-139.

Smith, B. T., Grossman, A. D. \& Walker, G. C. (2001) Visualization of mismatch repair in bacterial cells. Mol. Cell 8: 1197-1206.

Smith, B. T., Grossman, A. D. \& Walker, G. C. (2002) Localization of UvrA and effect of DNA damage on the chromosome of Bacillus subtilis. J. Bacteriol. 184: 488493.

Sonenshein, A. L. (2007) Control of key metabolic intersections in Bacillus subtilis. Nat. Rev. Microbiol. 5: 917-927.

Spies, M. A., Reese, J. G., Dodd, D., Pankow, K. L., Blanke, S. R. \& Baudry, J. (2009) Determinants of catalytic power and ligand binding in glutamate racemase. J. Am. Chem. Soc. 131: 5274-5284.

Stillman, T. J., Baker, P. J., Britton, K. L. \& Rice, D. W. (1993) Conformational flexibility in glutamate dehydrogenase. Role of water in substrate recognition and catalysis. J. Mol. Biol. 234: 1131-1139.

Stillman, T. J., Migueis, A. M., Wang, X. G., Baker, P. J., Britton, K. L., et al. (1999) Insights into the mechanism of domain closure and substrate specificity of glutamate dehydrogenase from Clostridium symbiosum. J. Mol. Biol. 285: 875-885.

Strauch, M. A., Aronson, A. I., Brown, S. W., Schreier, H. J. \& Sonenshein, A. L. (1988) Sequence of the Bacillus subtilis glutamine synthetase gene region. Gene 71: 257-265. 
Stülke, J., Martin-Verstraete, I., Zagorec, M., Rose, M., Klier, A. \& Rapoport, G. (1997) Induction of the Bacillus subtilis ptsGHI operon by glucose is controlled by a novel antiterminator, GlcT. Mol. Microbiol. 25: 65-78.

Sun, D. X. \& Setlow, P. (1991) Cloning, nucleotide sequence, and expression of the Bacillus subtilis ans operon, which codes for L-asparaginase and L-aspartase. J. Bacteriol. 173: 3831-3845.

Sun, D. X. \& Setlow, P. (1993) Cloning and nucleotide sequence of the Bacillus subtilis ansR gene, which encodes a repressor for the ans operon coding for Lasparaginase and L-aspartase. J. Bacteriol. 175: 2501-2506

Sung, H. M. \& Yasbin, R. E. (2002) Adaptive, or stationary-phase, mutagenesis, a component of bacterial differentiation in Bacillus subtilis. J. Bacteriol. 184: 5641-5653.

Tamburini, E. \& Mastromei, G. (2000) Do bacterial cryptic genes really exist? Res. Microbiol. 151: 179-182.

Tholen, S. (2008) Charakterisierung des gudB-Gens aus Bacillus subtilis. Diploma thesis. Georg-August-Universität Göttingen; Germany

Tiwari, A., Talcott, C., Knapp, M., Lincoln, P. \& Laderoute, K. (2007) Analyzing Pathways Using SAT-Based Approaches. In Algebraic Biology Springer Verlag Berlin/Heidelberg: 155-169.

Truglio, J. J., Croteau, D. L., Van Houten, B. \& Kisker, C. (2006) Prokaryotic nucleotide excision repair: the UvrABC system. Chem. Rev. 106: 233-252.

Vagin, A. A. \& Teplyakov, A. (1997) MOLREP: an automated program for molecular replacement. J. Appl. Crystallogr. 30: 1022-1025.

Veening, J-W., Smits, W. K., Hamoen, L. W., Jongbloed, J. D. \& Kuipers, O. P. (2004) Visualization of differential gene expression by improved cyan fluorescent protein and yellow fluorescent protein production in Bacillus subtilis. Appl. Environ. Microbiol. 70: 6809-6815. 
Verstee, S., Mogk, A. \& Schumann, W. (1999) The Bacillus subtilis htpG gene is not involved in thermal stress management. Mol. Gen. Genet. 1261: 582-588.

Viguera, E., Canceill, D. \& Ehrlich, S. D. (2001) Replication slippage involves DNA polymerase pausing and dissociation. EMBO J. 20: 2587-2595.

Wach, A. (1996) PCR-synthesis of marker cassettes with long flanking homology regions for gene disruptions in Saccharomyces cerevisiae. Yeast 12: 259-265.

Wacker, I., Ludwig, H., Reif, I., Blencke, H. M., Detsch, C. \& Stülke, J. (2003) The regulatory link between carbon and nitrogen metabolism in Bacillus subtilis: regulation of the gltAB operon by the catabolite control protein CcpA. Microbiology 149: 30013009.

Whatmore, A. M., Chudek, J. A. \& Reed, R. H. (1990) The effects of osmotic upshock on the intracellular solute pools of Bacillus subtilis. J. Gen. Microbiol. 136: 2527-2535.

Williams, A. N. \& Woessner, K. M. (2009) Monosodium glutamate 'allergy': menace or myth? Clin. Exp. Allergy 39: 640-646.

Witkin, E. M. (1956) Time, temperature, and protein synthesis: a study of ultravioletinduced mutation in bacteria. Cold Spring Harb. Symp. Quant. Biol. 21: 123-140.

Witkin, E. M. (1966) Radiation-induced mutations and their repair. Science 152: 1345 1353.

Wray, L. V., Jr., Atkinson, M. R. \& Fisher, S. H. (1994) The nitrogen-regulated Bacillus subtilis $n r g A B$ operon encodes a membrane protein and a protein highly similar to the Escherichia coli glnB-encoded PII protein. J. Bacteriol. 176: 108-114.

Wray, L. V., Jr., Ferson, A. E., Rohrer, K. \& Fisher, S. H. (1996) TnrA, a transcription factor required for global nitrogen regulation in Bacillus subtilis. Proc. Natl Acad. Sci. U.S.A. 93: 8841-8845. 
Wray, L. V., Jr., Zalieckas, J. M. \& Fisher, S. H. (2001) Bacillus subtilis glutamine synthetase controls gene expression through protein-protein interaction with transcription factor TnrA. Cell 107: 427-435.

Wray, L. V., Jr. \& Fisher, S. H. (2005) A feedback-resistant mutant of Bacillus subtilis glutamine synthetase with pleiotropic defects in nitrogen-regulated gene expression. J. Biol. Chem. 280: 33298-33304.

Wray, L. V., Jr. \& Fisher, S. H. (2010) Functional roles of the conserved Glu304 loop of Bacillus subtilis Glutamine Synthetase. . J. Bacteriol. 92: 5018-5025.

Yan, D. (2007) Protection of the glutamate pool concentration in enteric bacteria. Proc. Natl Acad. Sci. U.S.A. 104: 9475-9480.

Yanisch-Perron, C., Vieira, J. \& Messing, J. (1985) Improved M13 phage cloning vectors and host strains: nucleotide sequences of the M13mp18 and pUC19 vectors. Gene 33: 103-119.

Yip, K. S., Stillman, T. J., Britton, K. L., Artymiuk, P. J., Baker, P. J., et al. (1995) The structure of Pyrococcus furiosus glutamate dehydrogenase reveals a key role for ion-pair networks in maintaining enzyme stability at extreme temperatures. Structure 3: $1147-1158$.

Yoshida, K., Yamaguchi, H., Kinehara, M., Ohki, Y. H., Nakaura, Y. \& Fujita, Y. (2003) Identification of additional TnrA-regulated genes of Bacillus subtilis associated with a TnrA box. Mol. Microbiol. 49: 157-165.

Yuan, J., Doucette, C. D., Fowler, W. U., Feng, X. J., Piazza, M., et al. (2009) Metabolomics-driven quantitative analysis of ammonia assimilation in E. coli. Mol. Syst. Biol. 5: 302.

Zalieckas, J. M., Wray, L. V., Jr., Ferson, A. E. \& Fisher, S. H. (1998) Transcription-repair coupling factor is involved in carbon catabolite repression of the Bacillus subtilis hut and gnt operons. Mol. Microbiol. 27: 1031-1038. 
Zeigler, D. R., Prágai, Z., Rodriguez, S., Chevreux, B., Muffler, A., et al. (2008) The origins of 168, W23 and other Bacillus subtilis legacy strains. J. Bacteriol. 190: 69836995.

Zucker, M. (2003) Mfold web server for nucleic acid folding and hybridization prediction. Nucleic Acids Res. 31: 3406-3415. 


\section{Appendix}

\subsection{Oligonucleotides}

Oligonucleotides were purchased from Eurofins MWG Operon (Ebersberg, Germany) and Sigma Aldrich (Munich, Germany).

Tab. S1 - Oligonucleotides

\begin{tabular}{|c|c|c|}
\hline Name & Sequence $\left(5^{\prime} \rightarrow 3^{\prime}\right)^{\mathrm{a}-\mathrm{d}}$ & Description \\
\hline CD13 & $\begin{array}{l}\text { 5' AAACATATGGCTAGCTGGAGCCACCCGC } \\
\text { AGTTC }\end{array}$ & $\begin{array}{l}\text { sequencing of pGP172 } \\
\text { constructs, fwd }\end{array}$ \\
\hline FC146 & 5' CGATGCGTTCGCGATCCAGGC & $\begin{array}{l}\text { sequencing of pUT18 } \\
\text { constructs }\end{array}$ \\
\hline FC147 & 5' CCAGCCTGATGCGATTGCTGCAT & $\begin{array}{l}\text { sequencing of } \mathrm{p} 25-\mathrm{N} \\
\text { constructs }\end{array}$ \\
\hline FC148 & 5' GTCACCCGGATTGCGGCGG & $\begin{array}{l}\text { sequencing of pUT18C } \\
\text { constructs }\end{array}$ \\
\hline FC149 & 5' GCTGGCTTAACTATGCGGCATCAGA & $\begin{array}{l}\text { sequencing of pUT18C } \\
\text { constructs, }\end{array}$ \\
\hline FC150 & 5' GATTCGGTGACCGATTACCTGGC & $\begin{array}{l}\text { sequencing of pKT } 25 \\
\text { constructs }\end{array}$ \\
\hline FC151 & 5'CGCCAGGGTTTTCCCAGTCACG & $\begin{array}{l}\text { sequencing of pKT } 25 \\
\text { constructs }\end{array}$ \\
\hline HL47 & $\begin{array}{l}\text { 5' CTAATACGACTCACTATAGGGAGAGTCATA } \\
\text { GCGGGACATATAATG }\end{array}$ & $\begin{array}{l}\text { gapA probe, rev, } \\
\text { contains } \mathrm{T} 7 \text { promoter }\end{array}$ \\
\hline HL48 & 5' TATTCGAAAGAACCAAGTCAG & gapA probe, fwd \\
\hline HMB & 5' CGCGGATCCAGAAGATTCTAGGAGGTTA & sequencing of $g u d B$ \\
\hline 74 & $\mathrm{AC}$ & \\
\hline IR3 & 5' CGCGGATCCACGCCGATTCAGAAGACGA & sequencing of $g u d B$ \\
\hline IR6 & $\begin{array}{l}\text { 5' AAACGCGTCGACCTCCGATTTCGAGCTTT } \\
\text { TC A }\end{array}$ & sequencing of $g u d B$ \\
\hline JL7 & $\begin{array}{l}\text { 5' TTTGGATCCGTTCCCCCAGTTGTCAACAT } \\
\text { CAATAATTTTT }\end{array}$ & $\begin{array}{l}\text { ansA-lacZ fusion, rev } \\
\text { (BamHI) }\end{array}$ \\
\hline
\end{tabular}




\begin{tabular}{|c|c|c|}
\hline Name & Sequence $\left(5^{\prime} \rightarrow 3^{\prime}\right)^{\mathrm{a}-\mathrm{d}}$ & Description \\
\hline JS39 & 5' TCTATCAACAGGAGTCCAAGC & $\begin{array}{l}\text { Sequencing of } \\
\text { pWH844 constructs, } \\
\text { rev }\end{array}$ \\
\hline $\begin{array}{l}\text { kan- } \\
\text { fwd }\end{array}$ & 5' CAGCGAACCATTTGAGGTGATAGG & $\begin{array}{l}\text { LFH-PCR, } \\
\text { amplification of aphA3 } \\
\text { from pDG780, fwd }\end{array}$ \\
\hline $\begin{array}{l}\text { kan- } \\
\text { rev }\end{array}$ & 5' CGATACAAATTCCTCGTAGGCGCTCGG & $\begin{array}{l}\text { LFH-PCR, } \\
\text { amplification of aphA3 } \\
\text { from pDG780, rev }\end{array}$ \\
\hline $\begin{array}{l}\text { kan- } \\
\text { check } \\
\text { rev }\end{array}$ & 5' CTGCCTCCTCATCCTCTTCATCC & $\begin{array}{l}\text { LFH-PCR, sequencing } \\
\text { of the up-fragment }\end{array}$ \\
\hline $\begin{array}{l}\text { kan- } \\
\text { check } \\
\text { fwd }\end{array}$ & 5' CATCCGCAACTGTCCATACTCTG & $\begin{array}{l}\text { LFH-PCR, sequencing } \\
\text { of the down-fragment }\end{array}$ \\
\hline KG3 & $\begin{array}{l}\text { 5'AAAGGATCCCTGGTTCCGCGTGGTTCCAT } \\
\text { GTCAGCAAAGCAAGTCTCGAAAGATGAAG }\end{array}$ & $\begin{array}{l}\text { cloning of rocG into } \\
\text { pWH844, fwd (BamHI) } \\
\text { with thrombin cleavage } \\
\text { site }\end{array}$ \\
\hline KG4 & $\begin{array}{l}\text { 5' TTTAAGCTTTCATTAGACCCATCCGCGG } \\
\text { AAACGCGAT }\end{array}$ & $\begin{array}{l}\text { cloning of } \operatorname{roc} G \text { into } \\
\text { pWH844, rev (HindII) }\end{array}$ \\
\hline KG5 & 5' ATCAATCGAAGAGCAGAGGCATCTTCG & $\begin{array}{l}\text { LFH-PCR } \operatorname{roc} G \\
\text { (fwd up-fragment) }\end{array}$ \\
\hline KG6 & $\begin{array}{l}\text { 5'CCTATCACCTCAAATGGTTCGCTGGCTTCC } \\
\text { TTAATGATTGTTTGGGTAGACAG }\end{array}$ & $\begin{array}{l}\text { LFH-PCR rocG } \\
\text { (rev up-fragment) }\end{array}$ \\
\hline KG7 & $\begin{array}{l}\text { 5' CCGAGCGCCTACGAGGAATTTGTATCGGC } \\
\text { GTTTGGCGGCTTACATGACGG }\end{array}$ & $\begin{array}{l}\text { LFH-PCR } \operatorname{roc} G \\
\text { (fwd down-fragment) }\end{array}$ \\
\hline KG8 & 5' GGATGCAGCAAGGTCAAGATCAGCG & $\begin{array}{l}\text { LFH-PCR } \text { roc } G \\
\text { (rev down-fragment) }\end{array}$ \\
\hline KG9 & 5' CCGTGGGATGATGAGACGATCGG & $\begin{array}{l}\text { LFH-PCR rocG } \\
\text { (fwd up-fragment, } \\
\text { sequencing) }\end{array}$ \\
\hline
\end{tabular}




\begin{tabular}{|c|c|c|}
\hline Name & Sequence $\left(5^{\prime} \rightarrow 3^{\prime}\right)^{\mathrm{a}-\mathrm{d}}$ & Description \\
\hline KG10 & 5' GCGGGAGCCCGCAGAACACT & $\begin{array}{l}\text { LFH-PCR rocG } \\
\text { (rev down-fragment, } \\
\text { sequencing) }\end{array}$ \\
\hline KG11 & 5' CGCGAGCTTGACGGGGGAG & $\begin{array}{l}\text { amplification of ansR } \\
\text { region }\end{array}$ \\
\hline KG12 & $\begin{array}{l}\text { 5' CCTATCACCTCAAATGGTTCGCTGGATGG } \\
\text { CC AGCCGCTGAGTGAAG }\end{array}$ & $\begin{array}{l}\text { LFH-PCR ansR } \\
\text { (rev up-fragment) }\end{array}$ \\
\hline KG13 & $\begin{array}{l}\text { 5'CCGAGCGCCTACGAGGAATTTGTATCGCC } \\
\text { GAGAAGGTCAGCTGTATATTGAAGC }\end{array}$ & $\begin{array}{l}\text { LFH-PCR ansR } \\
\text { (fwd down-fragment) }\end{array}$ \\
\hline KG14 & 5' ACCTCGTAAATGCTCATGTCTTCGCC & $\begin{array}{l}\text { LFH-PCR ansR } \\
\text { (fwd up-fragment) }\end{array}$ \\
\hline KG15 & 5' CCGGAAGTCATTCTAGAGCTTGAGGA & $\begin{array}{l}\text { LFH-PCR ansR } \\
\text { (rev down-fragment) }\end{array}$ \\
\hline KG16 & 5' CTAGCGCCCACATCAATTTTGGCAC & $\begin{array}{l}\text { LFH-PCR ansR } \\
\text { (fwd up-fragment, } \\
\text { sequencing) }\end{array}$ \\
\hline KG17 & 5' GAAAGCCGGAGGAGGAGGAACC & $\begin{array}{l}\text { LFH-PCR ansR } \\
\text { (rev down-fragment, } \\
\text { sequencing) }\end{array}$ \\
\hline KG18 & $\begin{array}{l}\text { 5'AAAGGATCCCAGCTCAAGGTGAAAAAGG } \\
\text { AGCGGAA }\end{array}$ & $\begin{array}{l}\text { cloning of ansR into } \\
\text { pBQ200, fwd (BamHI) }\end{array}$ \\
\hline KG19 & $\begin{array}{l}\text { 5’TTTGTCGACTCATTAACTCAGTTCCTCCTG } \\
\text { TACTTTTCTTTTTGTG }\end{array}$ & $\begin{array}{l}\text { cloning of ans } R \text { into } \\
\text { pBQ200, rev (SalI) }\end{array}$ \\
\hline KG20 & $\begin{array}{l}\text { 5'AAAGAGCTCGATGGCAGCCGATCGAAAC } \\
\text { ACCG }\end{array}$ & $\begin{array}{l}\text { cloning of } g u d B \text { into } \\
\text { pGP172, fwd (SacI) }\end{array}$ \\
\hline KG21 & $\begin{array}{l}\text { 5'TTTGGATCCTCATTATATCCAGCCTCTAAA } \\
\text { ACGCGAAGCTT }\end{array}$ & $\begin{array}{l}\text { cloning of gudB into } \\
\text { pGP172, rev (BamHI) }\end{array}$ \\
\hline
\end{tabular}




\begin{tabular}{|c|c|c|}
\hline Name & Sequence $\left(5^{\prime} \rightarrow 3^{\prime}\right)^{\mathrm{a}-\mathrm{d}}$ & Description \\
\hline KG22 & 5' AGAAAAGGTTCGCGCTCGGCAC & sequencing of fur \\
\hline KG23 & 5' ССТTATTCTTATTTATATGAGCAGGACG & sequencing of $f_{s} r A$, fwd \\
\hline KG24 & 5' TTTGCAGCCGTGTGATGATACCC & sequencing of $f_{s r} A$, rev \\
\hline KG25 & 5' TTGAAGGGGAAAATGGGCTG & RT PCR ansA, fwd \\
\hline KG26 & 5' CTATTTCCACCCAGTATTCAGG & RT PCR ansA, rev \\
\hline KG27 & $\begin{array}{l}\text { 5' AAAGAATTCGCTGGCCATCAACAGAAA } \\
\text { TG }\end{array}$ & $\begin{array}{l}\text { translational ansA-lacZ } \\
\text { fusion, fwd (EcoRI) }\end{array}$ \\
\hline KG28 & 5' ATGGCTTGGACCCGTTATTGGGG & $\begin{array}{l}\text { LFH-PCR ans } A B \\
\text { (fwd up-fragment) }\end{array}$ \\
\hline KG29 & $\begin{array}{l}\text { 5' CCTATCACCTCAAATGGTTCGCTGGAGC } \\
\text { CA GCCCATTTTCСССТTC }\end{array}$ & $\begin{array}{l}\text { LFH-PCR ans } A B \\
\text { (rev up-fragment) }\end{array}$ \\
\hline KG30 & $\begin{array}{l}\text { 5' CCGAGCGCCTACGAGGAATTTGTATCG } \\
\text { CGGCGCTGATCATCTTGTTGATG }\end{array}$ & $\begin{array}{l}\text { LFH-PCR ans } A B \\
\text { (fwd down-fragment) }\end{array}$ \\
\hline KG31 & 5' AAGTCGGCACAACGCCTCCGG & $\begin{array}{l}\text { LFH-PCR ans } A B \\
\text { (rev down-fragment) }\end{array}$ \\
\hline KG32 & 5' GCGCATTCGTTGGGGAAAATCGG & $\begin{array}{l}\text { LFH-PCR ans } A B \text { (fwd } \\
\text { up-fragment, } \\
\text { sequencing) }\end{array}$ \\
\hline KG33 & 5' GCCGGCAGTTCCGACTGTTCC & $\begin{array}{l}\text { LFH-PCR ans } A B \\
\text { (rev down-fragment, } \\
\text { sequencing) }\end{array}$ \\
\hline KG34 & $\begin{array}{l}\text { 5‘AAATCTAGAGATGGAGGTGCGCCAACTGC } \\
\text { GTTAT }\end{array}$ & $\begin{array}{l}\text { cloning of } g l t C \text { into } \\
\text { B2H vectors, fwd } \\
(\mathrm{XbaI})\end{array}$ \\
\hline KG35 & $\begin{array}{l}\text { 5'TTTGGTACCCGTTGATACTGCTCCAGCTTA } \\
\text { GAGAAAAATTG }\end{array}$ & $\begin{array}{l}\text { cloning of } g l t C \text { into } \\
\text { B2H vectors, rev } \\
(\mathrm{KpnI})\end{array}$ \\
\hline
\end{tabular}




\begin{tabular}{|c|c|c|}
\hline Name & Sequence $\left(5^{\prime} \rightarrow 3^{\prime}\right)^{\text {a-d }}$ & Description \\
\hline KG36 & $\begin{array}{l}5^{`} \text { AAATCTAGAGATGTCAGCAAAGCAAGTC } \\
\text { TCGAAAGATGAAG }\end{array}$ & $\begin{array}{l}\text { cloning of } r o c G \text { into } \\
\text { B2H vectors, fwd } \\
\text { (XbaI) }\end{array}$ \\
\hline KG37 & $\begin{array}{l}\text { 5‘ TTTGGTACCCGGACCCATCCGCGGAAAC } \\
\text { GCGAT }\end{array}$ & $\begin{array}{l}\text { cloning of } \operatorname{roc} G \text { into } \\
\text { B2H vectors, rev } \\
\text { (KpnI) }\end{array}$ \\
\hline KG38 & 5' CCGTGTCGCATTAACACC & RT PCR ald, fwd \\
\hline KG39 & 5' ACCTGCTTCGGATCAGCA & RT PCR ald, rev \\
\hline KG40 & 5' TAAACCTTGGCGGCGGAA & RT PCR $b c d$, fwd \\
\hline KG41 & 5’ CCATATCCTCGACCGTTG & RT PCR $b c d$, rev \\
\hline KG42 & 5 GAAACGGCAAAACGTTCTGG & RT PCR rpsJ, fwd \\
\hline KG43 & 5 GTGTTGGGTTCACAATGTCG & RT PCR rpsJ, rev \\
\hline KG44 & 5 GCGTCGTATTGACCCAAGC & RT PCR rpsE, fwd \\
\hline KG45 & 5 TACCAGTACCGAATCCTACG & RT PCR rpsE, rev \\
\hline KG46 & $\begin{array}{l}\text { 5' AAGAATTCGATAAACCCAGCGAACCATT } \\
\text { TG }\end{array}$ & $\begin{array}{l}\text { aphA3 from pDG780, } \\
\text { fwd (EcoRI) }\end{array}$ \\
\hline KG47 & $\begin{array}{l}\text { 5' TTTCCCGGGATCGATACAAATTCCTCGTA } \\
\text { GGC }\end{array}$ & $\begin{array}{l}\text { aphA3 from pDG780, } \\
\text { rev (SmaI) }\end{array}$ \\
\hline KG48 & $\begin{array}{l}\text { 5' AAAGAATTCGATCCTTTAACTCTGGCAAC } \\
\text { CC }\end{array}$ & $\begin{array}{l}\text { erm } C \text { from } \mathrm{pDG} 647 \\
\text { fwd (EcoRI) }\end{array}$ \\
\hline KG49 & $\begin{array}{l}\text { 5' TTTCCCGGGGCCGACTGCGCAAAAGACA } \\
\text { TAAT }\end{array}$ & $\begin{array}{l}\text { ermC from pDG647, } \\
\text { rev (SmaI) }\end{array}$ \\
\hline KG50 & $\begin{array}{l}\text { 5' TTTATCGATGCGGCCGCAATGGTTTCTTA } \\
\text { GACGTCAGGTG }\end{array}$ & $\begin{array}{l}\text { amplification of bla } \\
\text { and } \text { ori from pUC18, } \\
\text { rev (ClaI, NotI) }\end{array}$ \\
\hline KG51 & $\begin{array}{l}\text { 5' AAAGAATTCGCTGTTTCCTGTGTGAAATT } \\
\text { GTTAT }\end{array}$ & $\begin{array}{l}\text { amplification of bla } \\
\text { and } \text { ori from pUC18, } \\
\text { fwd (EcoRI) }\end{array}$ \\
\hline KG52 & 5' GGTTATTGTCTCATGAGCGGAT & $\begin{array}{l}\text { sequencing of pGP882, } \\
\text { fwd in bla gene }\end{array}$ \\
\hline KG53 & 5' GCGCAACGCAATTAATGTGAG & $\begin{array}{l}\text { sequencing of pGP882, } \\
\text { rev in ori }\end{array}$ \\
\hline
\end{tabular}




\begin{tabular}{|c|c|c|}
\hline Name & Sequence $\left(5^{\prime} \rightarrow 3^{\prime}\right)^{\text {a-d }}$ & Description \\
\hline KG54 & $\begin{array}{l}\text { 5' AAA } \underline{\text { ATCGATCGTCGGTCTATTCAATTTAG }} \\
\text { TGAAT }\end{array}$ & $\begin{array}{l}\text { amplification lacA5', } \\
\text { fwd (ClaI) }\end{array}$ \\
\hline KG55 & $\begin{array}{l}\text { 5' TTTGTCGACGGATCCAGCTGATGGCATC } \\
\text { GACATGCTT }\end{array}$ & $\begin{array}{l}\text { amplification lacA5', } \\
\text { rev (BamHI, SalI) }\end{array}$ \\
\hline KG56 & $\begin{array}{l}\text { 5' AAAGTCGACCCCGGGCACCATATAAACT } \\
\text { GCTGATCGTC }\end{array}$ & $\begin{array}{l}\text { amplification lacA3', } \\
\text { fwd (SalI, SmaI) }\end{array}$ \\
\hline KG57 & $\begin{array}{l}\text { 5' TTTCAATTGATCATACGGGTGATTCCAGA } \\
\text { TG }\end{array}$ & $\begin{array}{l}\text { amplification lacA3' } \\
\text { rev (MfeI) }\end{array}$ \\
\hline KG58 & $\begin{array}{l}\text { 5' AAA } \underline{\text { AGATCTATCTTACATTGTAATCATGT }} \\
\text { CCAGAAAATGATC }\end{array}$ & $\begin{array}{l}\text { amplification } x y l R \text {, fwd } \\
\text { (BglII) }\end{array}$ \\
\hline KG59 & $\begin{array}{l}\text { 5' TTTCAATTGCCCGGGGTCGACGGATCCA T } \\
\text { GATTGTTTCCTCCTTTCAGATGCATTTTATTC } \\
\text { ATATAGTAAGTAC }\end{array}$ & $\begin{array}{l}\text { amplification PxylA } \\
\text { contains gapA } \\
\text { SD-sequence and ATG, } \\
\text { rev (BamHI, SalI, } \\
\text { SmaI, MfeI) }\end{array}$ \\
\hline KG60 & $\begin{array}{l}\text { 5' AAAAGATCTTCTAGAGGATCCGGTACCG } \\
\text { AATTCAGGTGGATCAGGCTCGGGATCTGGT } \\
\text { TCAATGGCCGACAAGGAGAAGAACG }\end{array}$ & $\begin{array}{l}\text { amplification yfP } \\
\text { C-terminal fragment, } \\
\text { fwd (BglII, XbaI, } \\
\text { BamHI, KpnI, EcoRI) }\end{array}$ \\
\hline KG61 & $\begin{array}{l}\text { 5' TTTGTCGACTTATCACTTGTACAGCTCGT } \\
\text { CCATGCC }\end{array}$ & $\begin{array}{l}\text { amplification yfP } \\
\text { C-terminal fragment, } \\
\text { rev (SalI) }\end{array}$ \\
\hline KG62 & $\begin{array}{l}\text { 5' AAAAGATCTCATGGCAGTAAAAGTCGGT } \\
\text { ATTAACGGTTTTGGTCGTATGGCCGACAAG } \\
\text { GAGAAGAACG }\end{array}$ & $\begin{array}{l}\text { amplification } y f P \\
\text { C-terminal fragment, } \\
\text { contains gapA SD- } \\
\text { sequence, fwd (BglII) }\end{array}$ \\
\hline KG63 & $\begin{array}{l}\text { 5' TTTGTCGACTCATTATGAATTCGGTACCG } \\
\text { GATCCTCTAGAGAACCAGATCCCGAGCCTG } \\
\text { ATCCACCTGACTTGTACAGCTCGTCCATGCC }\end{array}$ & $\begin{array}{l}\text { amplification } y f P \\
\text { C-terminal fragment, } \\
\text { rev, (SalI, XbaI, } \\
\text { BamHI, KpnI, EcoRI) }\end{array}$ \\
\hline
\end{tabular}




\begin{tabular}{|c|c|c|}
\hline Name & Sequence $\left(5^{\prime} \rightarrow 3^{\prime}\right)^{\mathrm{a}-\mathrm{d}}$ & Description \\
\hline KG64 & 5' TATCAGGGCCTCGACTACA & $\begin{array}{l}\text { sequencing of pGP882 } \\
\text { derivatives, fwd in } \\
\text { lacA5, }\end{array}$ \\
\hline KG65 & 5' CGCTGATTAAATACAGCATCGG & $\begin{array}{l}\text { sequencing of pGP882 } \\
\text { derivatives, rev lacA3, }\end{array}$ \\
\hline KG66 & $\begin{array}{l}\text { 5' GAAAATACTGACGAGGTTATATAAGATG } \\
\text { A }\end{array}$ & $\begin{array}{l}\text { sequencing of pGP884, } \\
\text { pGP885 derivatives, } \\
\text { fwd in PxylA }\end{array}$ \\
\hline KG67 & 5' CTCGATGTTGTGGCGGATCTT & $\begin{array}{l}\text { sequencing of pGP888, } \\
\text { rev in } y f p \text { C-terminal } \\
\text { fragment }\end{array}$ \\
\hline KG68 & 5' ATCACATGGTCCTGCTGGAGT & $\begin{array}{l}\text { sequencing of pGP889, } \\
\text { fwd in } y f p \text {-C- terminal } \\
\text { fragment }\end{array}$ \\
\hline KG69 & $\begin{array}{l}\text { 5'AAAAGATCTAGCGATATCCACTTCATCCA } \\
\text { CT }\end{array}$ & $\begin{array}{l}\text { amplification PxylA, } \\
\text { fwd (BgIII) }\end{array}$ \\
\hline KG70 & $\begin{array}{l}\text { 5' AAA } \underline{\text { ATCGATAGCTGTACGGAGAGGACAT }} \\
\text { TAT }\end{array}$ & $\begin{array}{l}\text { amplification } x k d E 5 \\
\text { fwd (ClaI) }\end{array}$ \\
\hline KG71 & $\begin{array}{l}\text { 5' TTTGTCGACGGATCCTTTCTAGCAGTATC } \\
\text { CGCTGTC }\end{array}$ & $\begin{array}{l}\text { amplification } x k d E 5^{\prime}, \\
\operatorname{rev}(\text { BamHI, SalI) }\end{array}$ \\
\hline KG72 & $\begin{array}{l}\text { 5’ AAAGTCGACCCCGGGTCAGGCTGACATT } \\
\text { AAAAGGACC }\end{array}$ & $\begin{array}{l}\text { amplification } x k d E 3 \text { ', } \\
\text { fwd (SalI, SmaI) }\end{array}$ \\
\hline KG73 & $\begin{array}{l}\text { 5' TTTCAATTGCAAGCATGTCTTCAACGAGC } \\
\text { TT }\end{array}$ & $\begin{array}{l}\text { amplification } x k d E 3 \text { ', } \\
\text { rev (MfeI) }\end{array}$ \\
\hline KG74 & $\begin{array}{l}\text { 5' AAAAGATCTTCTAGAGGATCCGGTACCG } \\
\text { AATTCAGGTGGATCAGGCTCGGGATCTGGT } \\
\text { TCAATGGTGAGCAAGGGCGAGGA }\end{array}$ & $\begin{array}{l}\text { amplification yfP } \\
\text { N-terminal fragment, } \\
\text { fwd (BglII, XbaI, } \\
\text { BamHI, KpnI, EcoRI) }\end{array}$ \\
\hline KG75 & $\begin{array}{l}\text { 5' TTTGTCGACTTATCACATGATATAGACGT } \\
\text { TGTGGCTGTTG }\end{array}$ & $\begin{array}{l}\text { amplification } y f P \\
\text { N-terminal fragment, } \\
\text { rev (SalI) }\end{array}$ \\
\hline
\end{tabular}




\begin{tabular}{|c|c|c|}
\hline Name & Sequence $\left(5^{\prime} \rightarrow 3^{\prime}\right)^{\mathrm{a}-\mathrm{d}}$ & Description \\
\hline KG76 & $\begin{array}{l}\text { 5' AAAAGATCTCATGGCAGTAAAAGTCGGT } \\
\text { ATTAACGGTTTTGGTCGTATGGTGAGCAAG } \\
\text { GGCGAGGA }\end{array}$ & $\begin{array}{l}\text { amplification } y f P \\
\text { C-terminal fragment, } \\
\text { contains gapA } \\
\text { SD-sequence, fwd } \\
\text { (BglII) }\end{array}$ \\
\hline KG77 & $\begin{array}{l}\text { 5' TTTGTCGACTCATTATGAATTCGGTACCG } \\
\text { GATCCTCTAGAGAACCAGATCCCGAGCCTG } \\
\text { ATCCACCTGACATGATATAGACGTTGTGGC } \\
\text { TGTTG }\end{array}$ & $\begin{array}{l}\text { amplification } y f P \\
\text { N-terminal fragment, } \\
\text { rev, (SalI, XbaI, } \\
\text { BamHI, KpnI, EcoRI) }\end{array}$ \\
\hline KG78 & 5' GCAGAAATAAGCTGCGTTCC & $\begin{array}{l}\text { sequencing of pGP883 } \\
\text { derivatives, fwd in } \\
x k d E 5\end{array}$ \\
\hline KG79 & 5' GAGCACCTTCGCAATCTCAA & $\begin{array}{l}\text { sequencing of pGP883 } \\
\text { derivatives, rev } x k d E 3 \text {, }\end{array}$ \\
\hline KG80 & 5' TCCAGCTCGACCAGGATGG & $\begin{array}{l}\text { sequencing of pGP886, } \\
\text { rev in } y f p \mathrm{~N} \text { - terminal } \\
\text { fragment }\end{array}$ \\
\hline KG81 & 5' GGGCACAAGCTGGAGTACAA & $\begin{array}{l}\text { sequencing of pGP887, } \\
\text { fwd in } y f p \mathrm{~N} \text {-terminal } \\
\text { fragment }\end{array}$ \\
\hline KG82 & 5' CCTTGGCGTCAAAGACCACG & sequencing of taq, rev \\
\hline KG83 & 5' ACGACGAGCTGGTCCTCGAG & sequencing of taq, fwd \\
\hline KG84 & $\begin{array}{l}\text { 5' AAAAGATCTATGGAATTCGGGATGCTGC } \\
\text { CC }\end{array}$ & $\begin{array}{l}\text { cloning of taq into } \\
\text { pWH844, fwd (BglII) }\end{array}$ \\
\hline KG85 & $\begin{array}{l}\text { 5' TTTGTCGACCTATCACTCCTTGGCGGAGA } \\
\text { G }\end{array}$ & $\begin{array}{l}\text { cloning of taq into } \\
\text { pWH844, rev (SalI) }\end{array}$ \\
\hline KG86 & 5' AGGATTCGCCATGCTTGTGA & $\begin{array}{l}\text { LFH-PCR } m f d \\
\text { (fwd up-fragment) }\end{array}$ \\
\hline KG87 & $\begin{array}{l}\text { 5' CCTATCACCTCAAATGGTTCGCTGCCTCG } \\
\text { TGTAAACCGTTGATG }\end{array}$ & $\begin{array}{l}\text { LFH-PCR } m f d \\
\text { (rev up-fragment) }\end{array}$ \\
\hline KG88 & $\begin{array}{l}\text { 5' CCGAGCGCCTACGAGGAATTTGTATCGG } \\
\text { GCATGCTGAAGGGCTTAAA }\end{array}$ & $\begin{array}{l}\text { LFH-PCR } m f d \\
\text { (fwd down-fragment) }\end{array}$ \\
\hline
\end{tabular}




\begin{tabular}{|c|c|c|}
\hline Name & Sequence $\left(5^{\prime} \rightarrow 3^{\prime}\right)^{\mathrm{a}-\mathrm{d}}$ & Description \\
\hline KG89 & 5' ATCCAACATCGCCGACAATG & $\begin{array}{l}\text { LFH-PCR } m f d \\
\text { (rev down-fragment) }\end{array}$ \\
\hline KG90 & 5' GCGGTTACGTCTTTTGTGCT & $\begin{array}{l}\text { LFH-PCR } m f d \\
\text { (fwd up-fragment, } \\
\text { sequencing) }\end{array}$ \\
\hline KG91 & 5' GAAATCCTGATGTGGACAGCA & $\begin{array}{l}\text { LFH-PCR } m f d \text { (rev } \\
\text { down-fragment, } \\
\text { sequencing) }\end{array}$ \\
\hline KG92 & $\begin{array}{l}\text { 5' TTTEGATCCTCATTATATCCAGCCTCTAA } \\
\text { AACGCG }\end{array}$ & $\begin{array}{l}\text { cloning of } g u d B \text { into } \\
\text { pAC5 (BamHI) }\end{array}$ \\
\hline KG93 & $\begin{array}{l}\text { 5'AAAGGATCCCTCGCAGGAAACAGAGAGG } \\
\text { A }\end{array}$ & $\begin{array}{l}\text { cloning of } m f d \text { into } \\
\text { pBQ200, fwd (BamHI) }\end{array}$ \\
\hline KG94 & $\begin{array}{l}\text { 5‘ TTTETCGACTCATTACGTTGATGAAATG } \\
\text { GTTTGCTTTTTC }\end{array}$ & $\begin{array}{l}\text { cloning of } m f d \text { into } \\
\text { pBQ200, rev (SalI) }\end{array}$ \\
\hline KG95 & 5' GTGGATTCAATCCGCAGCTT & $\begin{array}{l}\text { sequencing of } m f d \text {, fwd } \\
\text { (base 600) }\end{array}$ \\
\hline KG96 & 5' GGAACGTACGCAGAAATTGTC & $\begin{array}{l}\text { sequencing of } m f d \text {, fwd } \\
\text { (base 1251) }\end{array}$ \\
\hline KG97 & 5' GCTCCGTTCTATTCACGAAATC & $\begin{array}{l}\text { sequencing of } m f d \text {, fwd } \\
\text { (base 1884) }\end{array}$ \\
\hline KG98 & 5' GTAGAGGACATTGAGCGGAA & $\begin{array}{l}\text { sequencing of } m f d \text {, fwd } \\
\text { (base 2524) }\end{array}$ \\
\hline KG99 & 5' TTCGCTGTGTCTCCTTTACG & $\begin{array}{l}\text { sequencing of } m f d \text {, rev } \\
\text { (base } 3045 \text { ) }\end{array}$ \\
\hline KG100 & 5' GCAGCAATAACACCGGCAATAA & $\begin{array}{l}\text { LFH-PCR gudB } \\
\text { (fwd up-fragment) }\end{array}$ \\
\hline KG101 & $\begin{array}{l}\text { 5' CCTATCACCTCAAATGGTTCGCTGGCTG } \\
\text { GATATAAGTTGATGATTTGCAT }\end{array}$ & $\begin{array}{l}\text { LFH-PCR gudB } \\
\text { (rev up-fragment) }\end{array}$ \\
\hline KG102 & $\begin{array}{l}\text { 5' CCGAGCGCCTACGAGGAATTTGTATCGG } \\
\text { CTGAAATCAGCAGTTTTCCGT }\end{array}$ & $\begin{array}{l}\text { LFH-PCR } g u d B \\
\text { (fwd down-fragment) }\end{array}$ \\
\hline KG103 & 5' GCCATAATCCGGAGATTCATG & $\begin{array}{l}\text { LFH-PCR } g u d B \\
\text { (rev down-fragment) }\end{array}$ \\
\hline
\end{tabular}




\begin{tabular}{|c|c|c|}
\hline Name & Sequence $\left(5^{\prime} \rightarrow 3^{\prime}\right)^{\mathrm{a}-\mathrm{d}}$ & Description \\
\hline KG104 & 5' CGATTTCCGCTGCGATATGC & $\begin{array}{l}\text { LFH-PCR gudB } \\
\text { (fwd up-fragment, } \\
\text { sequencing) }\end{array}$ \\
\hline KG105 & 5' GGTTGATGATATCAGGATGGAG & $\begin{array}{l}\text { LFH-PCR gudB } \\
\text { (rev down-fragment, } \\
\text { sequencing) }\end{array}$ \\
\hline KG106 & $\begin{array}{l}5^{`} \text { AAAGGATCCATGTGGGAAAGTAAATTTT } \\
\text { CA AAAGAAGG }\end{array}$ & $\begin{array}{l}\text { cloning of guaB into } \\
\text { pGP380, fwd (BamHI) }\end{array}$ \\
\hline KG107 & $\begin{array}{l}\text { 5` TTTCTGCAGTCATTATGAAATTGTATAGT } \\
\text { TAGGTGATTCTTTTG }\end{array}$ & $\begin{array}{l}\text { cloning of guaB into } \\
\text { pGP380, rev (PstI) }\end{array}$ \\
\hline KG108 & $\begin{array}{l}\text { 5' AAAAGATCTGCGGTAGTACAGTAAGGAA } \\
\text { GGGGA }\end{array}$ & $\begin{array}{l}\text { cloning of gltB into } \\
\text { pGP1460 fwd, contains } \\
\text { SD-sequence (BgIII) }\end{array}$ \\
\hline KG109 & $\begin{array}{l}\text { 5' TTTGTCGACGGATCCTCATTACGGAAGA } \\
\text { ACTGAACTCCCCATCAA }\end{array}$ & $\begin{array}{l}\text { cloning of gltB into } \\
\text { pGP1460, rev, contains } \\
\text { stop codon (BamHI, } \\
\text { SalI) }\end{array}$ \\
\hline KG110 & $\begin{array}{l}\text { 5' AAAGGATCCATGGTTACATAAAAAGGGA } \\
\text { GGCTGAGAG }\end{array}$ & $\begin{array}{l}\text { cloning of yerD into } \\
\text { pGP1460, fwd, } \\
\text { contains SD-sequence } \\
\text { (BamHI) }\end{array}$ \\
\hline KG111 & $\begin{array}{l}\text { 5' TTTCTGCAGTCATTATGAAACAAATTGAT } \\
\text { GGATCAGGTTGTCG }\end{array}$ & $\begin{array}{l}\text { cloning of yerD into } \\
\text { pGP1460, rev, contains } \\
\text { stop codon (PstlI) }\end{array}$ \\
\hline KG112 & $\begin{array}{l}\text { 5' TTTGTCGACTGAAACAAATTGATGGATC } \\
\text { AGGTTGTCGATA }\end{array}$ & $\begin{array}{l}\text { cloning of yerD into } \\
\text { pGP1331 (SalI) }\end{array}$ \\
\hline KG113 & 5' GCGAGAAGCCATTTTATGTGAAGG & sequencing of yerD \\
\hline KG114 & 5' AGTCTCGAAATATCAGCCCCAG & $\begin{array}{l}\text { LFH-PCR } u v r A B \\
\text { (fwd up-fragment) }\end{array}$ \\
\hline KG115 & $\begin{array}{l}\text { 5' CCTATCACCTCAAATGGTTCGCTGAAGC } \\
\text { GTATACGGCGTTGAACCT }\end{array}$ & $\begin{array}{l}\text { LFH-PCR } u v r A B \text { (rev } \\
\text { up-fragment) }\end{array}$ \\
\hline
\end{tabular}




\begin{tabular}{|c|c|c|}
\hline Name & Sequence $\left(5^{\prime} \rightarrow 3^{\prime}\right)^{\mathrm{a}-\mathrm{d}}$ & Description \\
\hline KG116 & $\begin{array}{l}\text { 5' CCGAGCGCCTACGAGGAATTTGTATCGA } \\
\text { AG TGAATGCCAAGTTCCCGCT }\end{array}$ & $\begin{array}{l}\text { LFH-PCR } u v r A B \\
\text { (fwd down-fragment) }\end{array}$ \\
\hline KG117 & 5' CAAGAGCGATTTCATGCGTGTT & $\begin{array}{l}\text { LFH-PCR } u v r A B \\
\text { (rev down-fragment) }\end{array}$ \\
\hline KG118 & 5' GCGGCTAAGAAGAGAGGCATCGATA & gudB probe, fwd \\
\hline KG119 & $\begin{array}{l}\text { 5' P-GAACGTAACAGAAAAAGAGGTTAAGG } \\
\text { CTGTGAAGGCGCTTTCAATTTGG }\end{array}$ & mutagenesis: gudB $B_{m u t 1}$ \\
\hline KG120 & $\begin{array}{l}\text { 5' P-GAACGTAACAGAAAAAGAGGTTAAGG } \\
\text { CTGTTAAGGCTCTTTCAATTTGGATGAGTTT } \\
\text { AAAATGC }\end{array}$ & mutagenesis: $g u d B_{m u t 2}$ \\
\hline KG121 & 5' TATCCAGCCTCTAAAACGCGAAGCTTC & $\begin{array}{l}\text { amplification of } g u d B, \\
\text { rev, without stop codon } \\
\text { for C-terminal fusion to } \\
y f p\end{array}$ \\
\hline KG122 & $\begin{array}{l}\text { 5' GAAGCTTCGCGTTTTAGAGGCTGGATA } \\
\text { TGGTGAGCAAGGGCGAGGAGCT }\end{array}$ & $\begin{array}{l}\text { amplification of } y f p \text {, } \\
\text { fwd, for N-terminal } \\
\text { fusion to } g u d B \\
\text { (contains } \\
\text { complementary } \\
\text { sequence to } g u d B \text { ) }\end{array}$ \\
\hline KG123 & $\begin{array}{l}\text { 5' CTAATACGACTCACTATAGGGAGAAACGCC } \\
\text { G ACCATATATGCAGCGAG }\end{array}$ & $\begin{array}{l}\text { gudB probe, rev, } \\
\text { contains } \mathrm{T} 7 \text { promoter }\end{array}$ \\
\hline KG124 & $\begin{array}{l}\text { 5' AAAGGATCCGGGCATCCTGAAGAATTGC } \\
\text { ATGAGC }\end{array}$ & $\begin{array}{l}\text { cloning of yerD into } \\
\text { pGP1331, fwd } \\
\text { (BamHI) }\end{array}$ \\
\hline KG125 & $\begin{array}{l}\text { 5' AAAGGATCCGCAAAAGTTGTCGGCATCT } \\
\text { CAGATGC }\end{array}$ & $\begin{array}{l}\text { cloning of } g u d B \text { into } \\
\text { pGP1331, fwd } \\
\text { (BamHI) }\end{array}$ \\
\hline KG126 & $\begin{array}{l}\text { 5' TTTGTCGACTATCCAGCCTCTAAAACGC } \\
\text { GAAGCTTC }\end{array}$ & $\begin{array}{l}\text { cloning of gudB into } \\
\text { pGP1331, rev (SalI) }\end{array}$ \\
\hline KG127 & $5^{\prime}$ TCTAAACACGGTGCCTTTACAGGCC & $\begin{array}{l}\text { LFH-PCR mutSL } \\
\text { (fwd up-fragment) }\end{array}$ \\
\hline
\end{tabular}




\begin{tabular}{|c|c|c|}
\hline Name & Sequence $\left(5^{\prime} \rightarrow 3^{\prime}\right)^{\text {a-d }}$ & Description \\
\hline KG128 & $\begin{array}{l}\text { 5' CCTATCACCTCAAATGGTTCGCTGGGCAT } \\
\text { CCTGGTGCTCTGCCTTT }\end{array}$ & $\begin{array}{l}\text { LFH-PCR mutSL } \\
\text { (rev up-fragment) }\end{array}$ \\
\hline KG129 & $\begin{array}{l}\text { 5' CCGAGCGCCTACGAGGAATTTGTATCGG } \\
\text { CACATCAGACCCATTTACATGCCC }\end{array}$ & $\begin{array}{l}\text { LFH-PCR } m u t S L \\
\text { (fwd down-fragment) }\end{array}$ \\
\hline KG130 & 5' GGCTGATTCAAGGGTGCTGTTTGTAT & $\begin{array}{l}\text { LFH-PCR } m u t S L \\
\text { (rev down-fragment) }\end{array}$ \\
\hline KG131 & 5' CATCACATCAACCGGAGGCGAC & $\begin{array}{l}\text { LFH-PCR mutSL } \\
\text { (fwd up-fragment, } \\
\text { sequencing) }\end{array}$ \\
\hline KG132 & 5' TCCCCAGTGGAAAGGGCCTTTTTTG & $\begin{array}{l}\text { LFH-PCR mutSL (rev } \\
\text { down-fragment, } \\
\text { sequencing) }\end{array}$ \\
\hline KG133 & $\begin{array}{l}\text { 5' P-CAGAAAAAGAGGTGAAGGCGGTTAAG } \\
\text { GCTCTTTCAATTTGGATGAGTTT }\end{array}$ & mutagenesis: gudB $B_{m u t 3}$ \\
\hline $\begin{array}{l}\text { M13 } \\
\text { pUC } \\
\text { fwd }\end{array}$ & 5'GTAAAACGACGGCCAGTG- & $\begin{array}{l}\text { sequencing of pUC } \\
\text { derivatives, fwd }\end{array}$ \\
\hline $\begin{array}{l}\text { M13 } \\
\text { pUC } \\
\text { rev }\end{array}$ & 5' GGAAACAGCTATGACCATG & $\begin{array}{l}\text { sequencing of pUC } \\
\text { derivatives, rev }\end{array}$ \\
\hline $\begin{array}{l}\mathrm{mls} \\
\text { fwd } \\
\text { (kan) }\end{array}$ & $\begin{array}{l}\text { 5' CAGCGAACCATTTGAGGTGATAGGGATC } \\
\text { CTTTAACTCTGGCAACCCTC }\end{array}$ & $\begin{array}{l}\text { LFH-PCR, } \\
\text { amplification of ermC } \\
\text { from pDG647, fwd }\end{array}$ \\
\hline $\begin{array}{l}\text { mls rev } \\
\text { (kan) }\end{array}$ & $\begin{array}{l}\text { 5' CGATACAAATTCCTCGTAGGCGCTCGGG } \\
\text { CCGACTGCGCAAAAGACATAATCG }\end{array}$ & $\begin{array}{l}\text { LFH-PCR, } \\
\text { amplification of ermC } \\
\text { from pDG647, rev }\end{array}$ \\
\hline $\begin{array}{l}\text { mls- } \\
\text { check } \\
\text { rev }\end{array}$ & 5'GTTTTGGTCGTAGAGCACACGG & $\begin{array}{l}\text { LFH-PCR, sequencing } \\
\text { of the up-fragment }\end{array}$ \\
\hline $\begin{array}{l}\text { mls- } \\
\text { check }\end{array}$ & 5'CCTTAAAACATGCAGGAATTGACG & $\begin{array}{l}\text { LFH-PCR, sequencing } \\
\text { of the down-fragment }\end{array}$ \\
\hline
\end{tabular}




\begin{tabular}{|c|c|c|}
\hline Name & Sequence $\left(5^{\prime} \rightarrow 3^{\prime}\right)^{\text {a-d }}$ & Description \\
\hline NP20 & 5' GCAGCAGCCAACTCAGCTTCCTTTCGGGC & $\begin{array}{l}\text { sequencing of pGP172 } \\
\text { constructs, rev }\end{array}$ \\
\hline $\begin{array}{l}\mathrm{PAC} \\
5 \mathrm{~F}\end{array}$ & 5' GCGTAGCGAAAAATCCTTTTC & $\begin{array}{l}\text { sequencing of pAC } \\
\text { constructs, fwd }\end{array}$ \\
\hline $\begin{array}{l}\text { PAC } \\
5 \mathrm{R}\end{array}$ & 5' CTGCAAGCGATAAGTTGG & $\begin{array}{l}\text { sequencing of pAC } \\
\text { constructs, fwd }\end{array}$ \\
\hline PT05 & $\begin{array}{l}\text { 5' AAACGAGCTCGATGTCAGCAAAGCAAGT } \\
\text { CTCG }\end{array}$ & $\begin{array}{l}\text { cloning of } \operatorname{roc} G \text { into } \\
\text { pGP172, fwd (SacI) }\end{array}$ \\
\hline PT06 & $\begin{array}{l}\text { 5' CGCGGATCCTCATTAGACCCATCCGCGGA } \\
\text { AAC }\end{array}$ & $\begin{array}{l}\text { cloning of rocG into } \\
\text { pGP172, rev (BamHI) }\end{array}$ \\
\hline PT12 & $\begin{array}{l}\text { 5' CCCAAGCTTTCATTAGACCCATCCGCGGA } \\
\text { AAC }\end{array}$ & $\begin{array}{l}\text { cloning of } \operatorname{roc} G \text { into } \\
\text { pGP380, fwd (HindIII) }\end{array}$ \\
\hline $\begin{array}{l}\text { pWH } \\
844\end{array}$ & 5' TATGAGAGGATCGCATCACCAT & $\begin{array}{l}\text { sequencing of pWH844 } \\
\text { constructs, fwd }\end{array}$ \\
\hline $\begin{array}{l}\text { fwd } \\
r p s J- \\
\text { RT- } \\
\text { fwd }\end{array}$ & 5' GAAACGGCAAAACGTTCTGG & RT PCR rpsJ fwd \\
\hline $\begin{array}{l}\text { rpsJ- } \\
\text { RT- }\end{array}$ & 5' GTGTTGGGTTCACAATGTCG & RT PCR rpsJ rev \\
\hline rev & & \\
\hline $\begin{array}{l}\text { rpsE- } \\
\text { RT- } \\
\text { fwd }\end{array}$ & 5' GCGTCGTATTGACCCAAGC & RT PCR rpsE fwd \\
\hline $\begin{array}{l}\text { rpsE- } \\
\text { RT-rev }\end{array}$ & 5' TACCAGTACCGAATCCTACG & RT PCR rpsE rev \\
\hline ST1 & $\begin{array}{l}\text { 5' AAAGAATTCCATTCAGCTTTCAGAAAGC } \\
\text { TTACAGCGAATC }\end{array}$ & $\begin{array}{l}\text { gudB-lacZ furion, fwd } \\
\text { (EcoRI) }\end{array}$ \\
\hline ST2 & $\begin{array}{l}\text { 5' AAAGGATCCCCCAATTTTTCCAGAGCCT } \\
\text { TATGTATTACG }\end{array}$ & $\begin{array}{l}\text { gudB-lacZ furion, rev } \\
\text { (BamHI) }\end{array}$ \\
\hline ST3 & $\begin{array}{l}\text { 5' AAAGGATCCATGTCAGCAAAGCAAGTCT } \\
\text { CGAA }\end{array}$ & $\begin{array}{l}\text { cloning of rocG into } \\
\text { pGP380, fwd (BamHI) }\end{array}$ \\
\hline
\end{tabular}




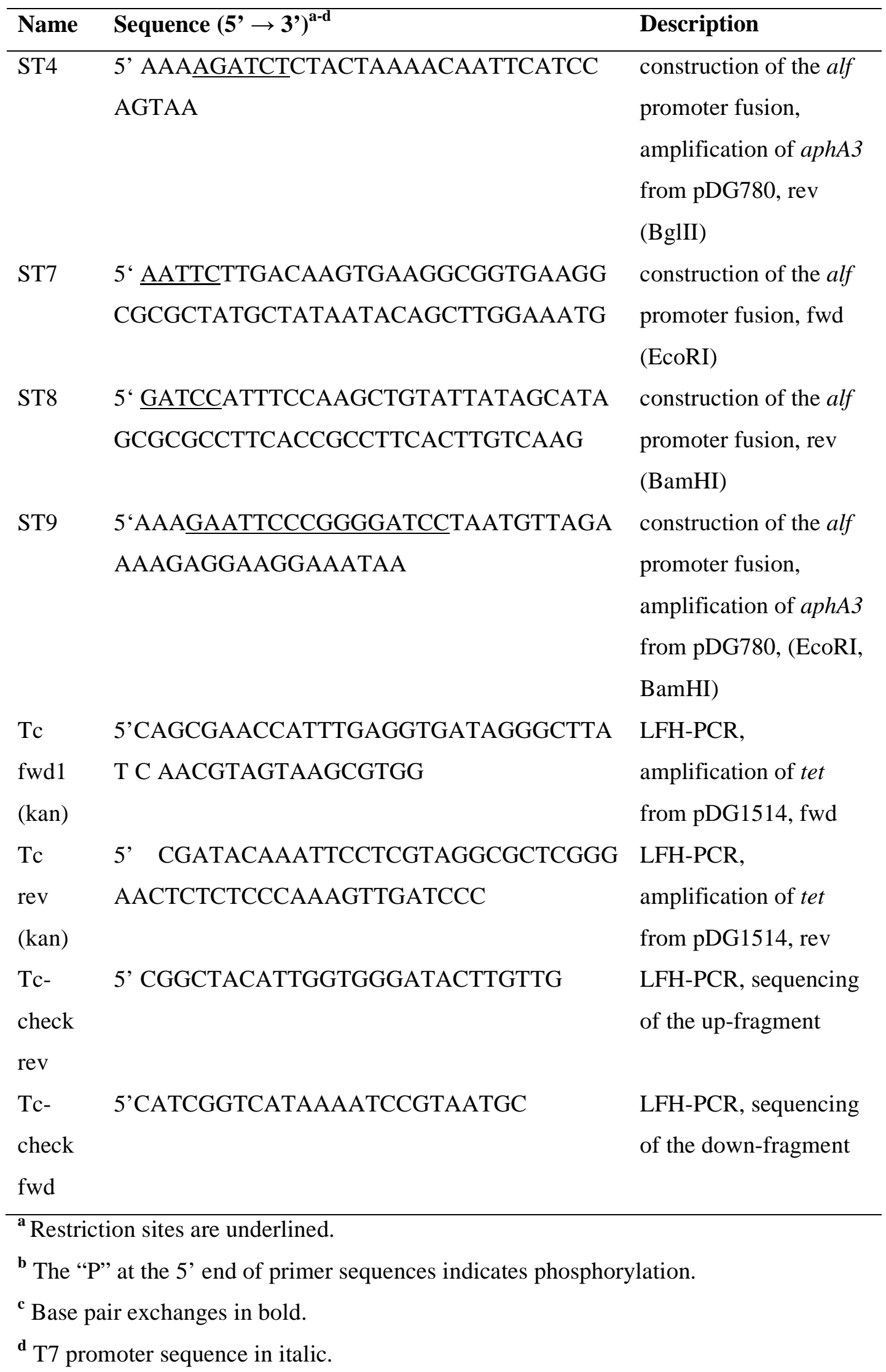




\subsection{Plasmids}

Tab. S2 - Plasmids

\begin{tabular}{|c|c|c|c|}
\hline Name & Relevant characteristics ${ }^{\mathrm{a}}$ & $\begin{array}{l}\text { Used } \\
\text { restriction sites }\end{array}$ & Reference \\
\hline pAC5 & $\begin{array}{l}\text { allows translational laZ promoter } \\
\text { fusions, homologous recombination } \\
\text { into the amyE site of B. subtilis } \\
\left(\mathrm{Cm}^{\mathrm{R}}\right) ; \text { E. coli } \mathrm{Amp}^{\mathrm{R}}\end{array}$ & & $\begin{array}{l}\text { Martin- } \\
\text { Verstraete et } \\
\text { al., } 1992\end{array}$ \\
\hline pAC6 & $\begin{array}{l}\text { allows transcriptional laZ promoter } \\
\text { fusions, contains SD-sequence and } \\
\text { ATG of } s a c B \text {, homologous } \\
\text { recombination into the amyE site of } \\
\text { B. subtilis }\left(\mathrm{Cm}^{\mathrm{R}}\right) \text {; E. coli Amp } \mathrm{A}^{\mathrm{R}}\end{array}$ & & $\begin{array}{l}\text { Stülke et al., } \\
1997\end{array}$ \\
\hline $\begin{array}{l}\text { pBlueskript } \\
\text { SK(-) }\end{array}$ & cloning vector & & $\begin{array}{l}\text { Stratagene, } \\
\text { Amsterdam, } \\
\text { Netherlands }\end{array}$ \\
\hline pBQ200 & $\begin{array}{l}\text { allows the overexpression of proteins } \\
\text { in B. subtilis; E. coli }\left(\mathrm{Amp}^{\mathrm{R}}\right) \text {, } \\
\text { B. subtilis }\left(\mathrm{Erm}^{\mathrm{R}}\right)\end{array}$ & & $\begin{array}{l}\text { Martin- } \\
\text { Verstraete et } \\
\text { al., } 1994\end{array}$ \\
\hline pDG647 & $\begin{array}{l}\text { template for erythromycin resistance } \\
\left(\mathrm{erm}^{\mathrm{C}}\right), \text { E. coli }\left(\mathrm{Amp}^{\mathrm{R}}\right)\end{array}$ & & $\begin{array}{l}\text { Guérout- } \\
\text { Fleury et al., } \\
1995\end{array}$ \\
\hline pDG780 & $\begin{array}{l}\text { template for kanamycin resistance } \\
(\operatorname{aphA3}), \text { E. coli }\left(\mathrm{Amp}^{\mathrm{R}}\right)\end{array}$ & & $\begin{array}{l}\text { Guérout- } \\
\text { Fleury et al., } \\
1995\end{array}$ \\
\hline pDG1514 & $\begin{array}{l}\text { template for tetracycline resistance } \\
\left(\text { tet }^{C}\right), \text { E. coli }\left(\mathrm{Amp}^{\mathrm{R}}\right)\end{array}$ & & $\begin{array}{l}\text { Guérout- } \\
\text { Fleury et al., } \\
1995\end{array}$ \\
\hline pGP172 & $\begin{array}{l}\text { allows expression of proteins } \\
\text { carrying a Strep-tag at their } \\
\text { N-terminus in } E \text {. coli } \\
\text { BL21(DE3)/pLysS }\left(\mathrm{Amp}^{\mathrm{R}}\right)\end{array}$ & & $\begin{array}{l}\text { Merzbacher et } \\
\text { al., } 2004\end{array}$ \\
\hline
\end{tabular}




\begin{tabular}{|c|c|c|c|}
\hline Name & Relevant characteristics ${ }^{\mathrm{a}}$ & $\begin{array}{l}\text { Used } \\
\text { restriction sites }\end{array}$ & Reference \\
\hline pGP380 & $\begin{array}{l}\text { Allows overexpression of N-terminal } \\
\text { Strep-tag fusion proteins in } \\
\text { B. subtilis }\left(\mathrm{Erm}^{\mathrm{R}}\right) ; \text { E. coli }\left(\mathrm{Amp}^{\mathrm{R}}\right) \text {; }\end{array}$ & & $\begin{array}{l}\text { Herzberg et } \\
\text { al., } 2007\end{array}$ \\
\hline pGP526 & $\begin{array}{l}\text { translational gltA-lacZ fusion in } \\
\text { pAC7 }\end{array}$ & & $\begin{array}{l}\text { Wacker et al., } \\
2003\end{array}$ \\
\hline pGP529 & pBQ200-rocG & & $\begin{array}{l}\text { Commichau et } \\
\text { al., } 2008\end{array}$ \\
\hline pGP651 & $\begin{array}{l}\text { translational gudB-lacZ fusion in } \\
\text { pAC5 (ST1/ST2) }\end{array}$ & BamHI/EcoRI & $\begin{array}{l}\text { Gunka et al. } \\
\text { subm. }\end{array}$ \\
\hline pGP652 & $\begin{array}{l}\text { pGP380-rocG-SR3 (G277A) } \\
\text { (ST3/PT12) }\end{array}$ & BamHI/HindIII & Tholen, 2008 \\
\hline pGP653 & $\begin{array}{l}\text { promoterless aphA3 gene in pAC6 } \\
\text { (ST9/ST4), allows promoter fusion } \\
\text { to a aphA3-lacZ operon }\end{array}$ & BglII/EcoRI & $\begin{array}{l}\text { Gunka et al. } \\
\text { subm. }\end{array}$ \\
\hline pGP655 & $\begin{array}{l}\text { artificial alf promoter system in } \\
\text { pGP653 (ST7/ST8) }\end{array}$ & EcoRI/BamHI & $\begin{array}{l}\text { Gunka et al. } \\
\text { subm. }\end{array}$ \\
\hline pGP852 & $\begin{array}{l}\text { pBQ200- rocG-SR1 (T923C), } \\
\text { mutagenesis of pGP529 in XL1-Red }\end{array}$ & & $\begin{array}{l}\text { Gunka et al., } \\
2010\end{array}$ \\
\hline pGP853 & $\begin{array}{l}\text { pBQ200-rocG-SR2 }(C 702 A) \\
\text { mutagenesis of pGP529 in XL1-Red }\end{array}$ & & $\begin{array}{l}\text { Gunka et al., } \\
2010\end{array}$ \\
\hline pGP854 & $\begin{array}{l}\text { pBQ200-rocG-SR3 }(G 277 A) \\
\text { mutagenesis of pGP529 in XL1-Red }\end{array}$ & & $\begin{array}{l}\text { Gunka et al., } \\
2010\end{array}$ \\
\hline pGP855 & $\begin{array}{l}\text { pBQ200-rocG-SR4 }(G 997 A) \\
\text { mutagenesis of pGP529 in XL1-Red }\end{array}$ & & $\begin{array}{l}\text { Gunka et al., } \\
2010\end{array}$ \\
\hline pGP856 & pGP172-rocG-SR1 (T923C) & SacI/BamHI & $\begin{array}{l}\text { Gunka et al., } \\
2010\end{array}$ \\
\hline pGP857 & pGP172-rocG-SR2 (C702A) & SacI/BamHI & $\begin{array}{l}\text { Gunka et al., } \\
2010\end{array}$ \\
\hline pGP858 & pGP172-rocG-SR3 (G277A) & $\mathrm{SacI} / \mathrm{BamHI}$ & $\begin{array}{l}\text { Gunka et al., } \\
2010\end{array}$ \\
\hline
\end{tabular}




\begin{tabular}{|c|c|c|c|}
\hline Name & Relevant characteristics $^{\mathrm{a}}$ & $\begin{array}{l}\text { Used } \\
\text { restriction sites }\end{array}$ & Reference \\
\hline pGP859 & pGP172-rocG-SR4 (G997A) & SacI/BamHI & $\begin{array}{l}\text { Gunka et al., } \\
2010\end{array}$ \\
\hline pGP860 & $\begin{array}{l}\text { pWH844-rocG }(\mathrm{KG} 3 / \mathrm{KG} 4) \text {, contains } \\
\text { thrombin cleavage site }\end{array}$ & BamHI/HindIII & This work \\
\hline pGP861 & $\begin{array}{l}\text { pWH844-rocG-SR3 (G277A) } \\
\text { (KG3/KG4), contains thrombin } \\
\text { cleavage site }\end{array}$ & BamHI/HindIII & This work \\
\hline pGP862 & $\begin{array}{l}\text { pWH844-rocG-MF (G364A) } \\
\text { (KG3/KG4), contains thrombin } \\
\text { cleavage site }\end{array}$ & BamHI/HindIII & This work \\
\hline pGP863 & pGP172-gudB (KG20/KG21) & $\mathrm{SacI} / \mathrm{BamHI}$ & $\begin{array}{l}\text { Gunka et al., } \\
2010\end{array}$ \\
\hline pGP864 & pGP172-gudB1 (KG20/KG21) & $\mathrm{SacI} / \mathrm{BamHI}$ & $\begin{array}{l}\text { Gunka et al., } \\
2010\end{array}$ \\
\hline pGP865 & $\begin{array}{l}\text { pGP172-rocG-MF (G364A) } \\
\text { (PT5/PT6) }\end{array}$ & SacI/BamHI & $\begin{array}{l}\text { Gunka et al., } \\
2010\end{array}$ \\
\hline pGP866 & $\begin{array}{l}\text { pGP172-rocG-SR5 }(T 472 C) \\
\text { (PT5/PT6) }\end{array}$ & SacI/BamHI & $\begin{array}{l}\text { Gunka et al., } \\
2010\end{array}$ \\
\hline pGP867 & $\begin{array}{l}\text { pBQ200-rocG-SR6 }(G 907 A) \\
\text { mutagenesis of pGP529 in XL1-Red }\end{array}$ & & $\begin{array}{l}\text { Gunka et al., } \\
2010\end{array}$ \\
\hline pGP868 & $\begin{array}{l}\text { pBQ200-rocG-SR7 }(G 904 A) \\
\text { mutagenesis of pGP529 in XL1-Red }\end{array}$ & & $\begin{array}{l}\text { Gunka et al., } \\
2010\end{array}$ \\
\hline pGP869 & $\begin{array}{l}\text { pBQ200-rocG-SR8 }(A 304 G) \\
\text { mutagenesis of pGP529 in XL1-Red }\end{array}$ & & $\begin{array}{l}\text { Gunka et al., } \\
2010\end{array}$ \\
\hline pGP870 & $\begin{array}{l}\text { pBQ200-rocG-SR9 }(A 479 G) \\
\text { mutagenesis of pGP529 in XL1-Red }\end{array}$ & & $\begin{array}{l}\text { Gunka et al., } \\
2010\end{array}$ \\
\hline pGP871 & $\begin{array}{l}\text { pBQ200-rocG-SR10 }(G 831 A A) \\
\text { mutagenesis of pGP529 in XL1-Red }\end{array}$ & & $\begin{array}{l}\text { Gunka et al., } \\
2010\end{array}$ \\
\hline pGP872 & $\begin{array}{l}\text { ansA-lacZ fusion in pAC5 } \\
(\mathrm{KG} 27 / \mathrm{JL} 7)\end{array}$ & BamHI/EcoRI & This work \\
\hline
\end{tabular}




\begin{tabular}{|c|c|c|c|}
\hline Name & Relevant characteristics $^{\mathrm{a}}$ & $\begin{array}{l}\text { Used } \\
\text { restriction sites }\end{array}$ & Reference \\
\hline pGP873 & pBQ200-ansR (KG18/19) & BamHI/SalI & This work \\
\hline pGP874 & pUT18-gltC (KG34/KG35) & $\mathrm{XbaI} / \mathrm{KpnI}$ & This work \\
\hline pGP875 & pUT18C-gltC (KG34/KG35) & $\mathrm{XbaI} / \mathrm{KpnI}$ & This work \\
\hline pGP876 & p25-N-gltC (KG34/KG35) & $\mathrm{XbaI} / \mathrm{KpnI}$ & This work \\
\hline pGP877 & pKT25-gltC (KG34/KG35) & $\mathrm{XbaI} / \mathrm{KpnI}$ & This work \\
\hline pGP878 & pUT18-rocG (KG36/KG37) & $\mathrm{XbaI} / \mathrm{KpnI}$ & This work \\
\hline pGP879 & pUT18C-rocG (KG36/KG37) & $\mathrm{XbaI} / \mathrm{KpnI}$ & This work \\
\hline pGP880 & p25-N-rocG (KG36/KG37) & $\mathrm{XbaI} / \mathrm{KpnI}$ & This work \\
\hline pGP881 & pKT25-rocG (KG36/KG37) & $\mathrm{XbaI} / \mathrm{KpnI}$ & This work \\
\hline pGP882 & $\begin{array}{l}\text { ori-bla from pUC18 (KG50/51), } \\
\text { lacA5‘(KG54/KG55), } \\
\text { lacA3‘(KG56/KG56); E. coli } \mathrm{Amp}^{\mathrm{R}}\end{array}$ & $*$ & This work \\
\hline pGP883 & $\begin{array}{l}\text { ori-bla from pUC18 (KG50/51), } \\
x k d E 5^{\prime}(\mathrm{KG} 70 / 71), \\
x k d E 3^{\prime}(\mathrm{KG} 72 / \mathrm{KG} 73) ; \text { E. coli } \mathrm{Amp}^{\mathrm{R}}\end{array}$ & $*$ & This work \\
\hline pGP884 & $\begin{array}{l}\text { pGP882-xylR+PxylA (KG58/KG59) } \\
\text { aphA3 (KG46/47); E. coli Amp }{ }^{\mathrm{R}}\end{array}$ & $*$ & This work \\
\hline pGP885 & $\begin{array}{l}\text { pGP883- PxylA (KG69/59)-ermC } \\
(\mathrm{KG} 48 / 49) ; \text { E. coli Amp }{ }^{\mathrm{R}}\end{array}$ & $*$ & This work \\
\hline pGP886 & $\begin{array}{l}\text { pGP883-yfp N-terminus for } \\
\text { C-terminal fusions (KG74/KG75), } \\
\text { allows homologous recombination } \\
\text { into the } x k d E \text { site of } B \text {. subtilis } \\
\left(\mathrm{Erm}^{\mathrm{R}}\right)\end{array}$ & BglII, SalI & This work \\
\hline pGP887 & $\begin{array}{l}\text { pGP883- } y f p \text { N-terminus for } \\
\text { N-terminal fusions (KG76/KG77), } \\
\text { allows homologous recombination } \\
\text { into the } x k d E \text { site of B. subtilis } \\
\left(\mathrm{Erm}^{\mathrm{R}}\right)\end{array}$ & BglII, SalI & This work \\
\hline
\end{tabular}




\begin{tabular}{|c|c|c|c|}
\hline Name & Relevant characteristics ${ }^{\mathrm{a}}$ & $\begin{array}{l}\text { Used } \\
\text { restriction sites }\end{array}$ & Reference \\
\hline pGP888 & $\begin{array}{l}\text { pGP882-yfp C-terminus for } \\
\text { C-terminal fusions (KG60/KG61), } \\
\text { allows homologous recombination } \\
\text { into the lacA site of B. subtilis } \\
\left(\mathrm{Kan}^{\mathrm{R}}\right)\end{array}$ & BglII, SalI & This work \\
\hline pGP889 & $\begin{array}{l}\text { pGP882- } y f p \text { C-terminus for } \\
\text { N-terminal fusions (KG62/KG63), } \\
\text { allows homologous recombination } \\
\text { into the lacA site of B. subtilis } \\
\left(\mathrm{Kan}^{\mathrm{R}}\right)\end{array}$ & BglII, SalI & This work \\
\hline pGP890 & pGP886-gltC & $\mathrm{XbaI} / \mathrm{KpnI}$ & This work \\
\hline pGP891 & pGP888-gltC & $\mathrm{XbaI} / \mathrm{KpnI}$ & This work \\
\hline pGP892 & pGP887-gltC & $\mathrm{XbaI} / \mathrm{KpnI}$ & This work \\
\hline pGP893 & pGP889-gltC & $\mathrm{XbaI} / \mathrm{KpnI}$ & This work \\
\hline pGP894 & pGP886-rocG & $\mathrm{XbaI} / \mathrm{KpnI}$ & This work \\
\hline pGP895 & pGP888-rocG & $\mathrm{XbaI} / \mathrm{KpnI}$ & This work \\
\hline pGP896 & pGP887-rocG & $\mathrm{XbaI} / \mathrm{KpnI}$ & This work \\
\hline pGP897 & pGP889-rocG & $\mathrm{XbaI} / \mathrm{KpnI}$ & This work \\
\hline pGP898 & $\begin{array}{l}\text { pGP884-yfp for C-terminal } \\
\text { (KG61/KG74) fusions allows } \\
\text { homologous recombination into the } \\
\text { lacA site of B. subtilis }\left(\operatorname{Kan}^{\mathrm{R}}\right)\end{array}$ & SalI/MfeI & This work \\
\hline pGP900 & $g u d B$ in $\mathrm{pAC} 5$ (ST01/KG92) & EcoRI/BamHI & $\begin{array}{l}\text { Gunka et al., } \\
\text { subm. }\end{array}$ \\
\hline pGP901 & pGP380-guaв (KG106/107) & (BamHI/PstI) & This work \\
\hline pGP919 & gltA-lacZ fusion in pAC5 & & $\begin{array}{l}\text { Commichau et } \\
\text { al., 2007b }\end{array}$ \\
\hline pGP932 & $\begin{array}{l}\text { pBQ200-rocG-MF (G364A) } \\
\text { mutagenesis of pGP529 in XL1-Red }\end{array}$ & & $\begin{array}{l}\text { Gunka et al., } \\
2010\end{array}$ \\
\hline pGP933 & $\begin{array}{l}\text { pBQ200-rocG-SR5 }(T 472 C) \\
\text { mutagenesis of pGP529 in XL1-Red }\end{array}$ & & $\begin{array}{l}\text { Gunka et al., } \\
2010\end{array}$ \\
\hline
\end{tabular}




\begin{tabular}{|c|c|c|c|}
\hline Name & Relevant characteristics $^{\mathrm{a}}$ & $\begin{array}{l}\text { Used } \\
\text { restriction sites }\end{array}$ & Reference \\
\hline pGP1331 & $\begin{array}{l}\text { pUS19-3xFLAG, integrate by single } \\
\text { crossing-over into B. subtilis } \\
\text { chromosome; allows fusion to a } \\
\text { 3xFLAG at the C-terminus; E. coli } \\
\left(\mathrm{Amp}^{\mathrm{R}}\right), \text { B. subtilis }\left(\text { Spec }^{\mathrm{R}}\right)\end{array}$ & & $\begin{array}{l}\text { Lehnik- } \\
\text { Habrink et al., } \\
2010\end{array}$ \\
\hline pGP1702 & $\begin{array}{l}\text { pBluescript (SK-)-rocG-SR3 } \\
(G 277 A)\end{array}$ & $\mathrm{XbaI} / \mathrm{KpnI}$ & This work \\
\hline pGP1703 & pBluescript (SK-)-rocG-MF (G364A) & $\mathrm{XbaI} / \mathrm{KpnI}$ & This work \\
\hline pGP1708 & $\begin{array}{l}\text { pGP380-rocG-MF (G364A) } \\
\text { (ST3/PT12) }\end{array}$ & BamHI/HindIII & This work \\
\hline pGP1709 & pGP380-rocG (ST3/PT12) & BamHI/HindIII & This work \\
\hline pGP1714 & $\begin{array}{l}\text { gudB mut1 in pAC5 } \\
\text { (ST01/KG119/KG92) }\end{array}$ & EcoRI/BamHI & $\begin{array}{l}\text { Gunka et al., } \\
\text { subm. }\end{array}$ \\
\hline pGP1715 & $\begin{array}{l}\operatorname{gudB}_{\text {mut2 }} \text { in pAC5 } \\
\text { (ST01/KG120/KG92) }\end{array}$ & EcoRI/BamHI & $\begin{array}{l}\text { Gunka et al., } \\
\text { subm. }\end{array}$ \\
\hline pGP1720 & $\begin{array}{l}\text { pGP1331-gudB (3xFLAG) } \\
(\text { KG125/KG126) }\end{array}$ & BamHI/SalI & This work \\
\hline pGP1721 & $\begin{array}{l}\text { gudB mut3 in pAC5 } \\
\text { (ST01/KG133/KG92) }\end{array}$ & EcoRI/BamHI & $\begin{array}{l}\text { Gunka et al., } \\
\text { subm. }\end{array}$ \\
\hline pIYFP & $\begin{array}{l}\text { template for } y f p \text { gene, } B . \text { subtilis } \\
\left(\mathrm{Cm}^{\mathrm{R}}\right), \text { E. coli }\left(\mathrm{Amp}^{\mathrm{R}}\right)\end{array}$ & & $\begin{array}{l}\text { Veening et } \\
\text { al., } 2004\end{array}$ \\
\hline $\mathrm{p} 25-\mathrm{N}$ & $P_{l a c}-\operatorname{mcs}-c y a A\left(\operatorname{Kan}^{\mathrm{R}}\right)$ & & $\begin{array}{l}\text { Claessen } \text { et al., } \\
2008\end{array}$ \\
\hline pKT25 & $P_{l a c}-c y a A-\operatorname{mcs}\left(\operatorname{Kan}^{\mathrm{R}}\right)$ & & $\begin{array}{l}\text { Karimova et } \\
\text { al., } 1998\end{array}$ \\
\hline pKT25::zip & $P_{l a c}-c y a A-z i p\left(\operatorname{Kan}^{\mathrm{R}}\right)$ & & $\begin{array}{l}\text { Karimova et } \\
\text { al., } 1998\end{array}$ \\
\hline pUT18 & $P_{l a c}-\mathrm{mcs}-c y a A\left(\mathrm{Amp}^{\mathrm{R}}\right)$ & & $\begin{array}{l}\text { Karimova et } \\
\text { al., } 1998\end{array}$ \\
\hline pUT18C & $P_{l a c}-c y a A-m c s\left(\mathrm{Amp}^{\mathrm{R}}\right)$ & & $\begin{array}{l}\text { Karimova et } \\
\text { al., } 1998\end{array}$ \\
\hline
\end{tabular}




\begin{tabular}{llll}
\hline Name & Relevant characteristics $^{\text {a }}$ & $\begin{array}{l}\text { Used } \\
\text { restriction sites }\end{array}$ & Reference \\
\hline pUT18C:: & Plac $_{\text {-cyaA-zip }\left(\mathrm{Amp}^{\mathrm{R}}\right)}$ & Karimova et \\
zip & & al., 1998 \\
pUC18 & cloning vector & Yanisch- \\
& & Perron, et al., \\
& & 1985 \\
pWH844 & allows expression of proteins & Schirmer et \\
& carrying a His tag at their N-terminus & al., 1997 \\
& in E. coli $\left(\mathrm{Amp}^{\mathrm{R}}\right)$ & \\
\hline
\end{tabular}

${ }^{\mathbf{a}}$ Resistance abbreviations as follows: Amp, ampicillin; $\mathrm{Cm}$, chloramphenicol; Erm, erythromycin; Kan, kanamycin; Spec, spectinomycin

* constructed by three fragment ligation 


\subsection{Strains}

Tab. S3 - Strains

\begin{tabular}{|c|c|c|}
\hline Strain & Genotype $^{\text {a }}$ & Reference/Construction $^{b}$ \\
\hline \multicolumn{3}{|c|}{ Bacillus subtilis } \\
\hline 168 & $\operatorname{trpC2}$ & Laboratory collection \\
\hline BD3349 & BD3349 trpC2 ypbH::spc & Persuh et al. 2002 \\
\hline GP28 & $\begin{array}{l}\operatorname{trp} C 2 \text { agudB::cat rocG }:: \operatorname{Tn} 10 \text { spc } \\
\text { amyE::(gltA-lacZ aphA3) }\end{array}$ & Commichau et al., 2007a \\
\hline GP655 & $\operatorname{trpC} 2$ $\triangle$ rocF $:: a p h A 3$ & Commichau, 2006 \\
\hline GP656 & $\operatorname{trpC2} \Delta r o c D:: a p h A 3$ & Commichau, 2006 \\
\hline GP669 & $\operatorname{trp} C 2$ amyE $::\left(g l t A^{\prime}-{ }^{\prime} l a c Z\right.$ cat $)$ & Commichau et al., 2006 \\
\hline GP717 & 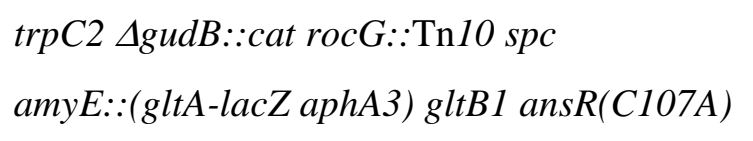 & Commichau et al., 2008 \\
\hline GP738 & $\operatorname{trp} C 2$ gltC:: $\operatorname{Tn} 10 \mathrm{spc}$ & Commichau, 2006 \\
\hline GP747 & $\operatorname{trp} C 2 \operatorname{roc} G:: \operatorname{Tn} 10 \operatorname{spc}$ & Commichau et al., 2007b \\
\hline GP753 & $\operatorname{trp} C 2 \operatorname{roc} G:: \operatorname{Tn} 10$ spc gudB1 & $\begin{array}{l}\text { Gunka } \text { et al., subm.; } \\
\text { spontaneous mutation of } \\
\text { GP747 on SP }\end{array}$ \\
\hline GP754 & $\operatorname{trpC} 2$ rocG::cat amyE::(gltA-lacZ aphA3) & Commichau et al., 2007a \\
\hline GP801 & $\begin{array}{l}\operatorname{trpC} 2 \text { rocG::cat gudB1 amyE::(gltA-lacZ } \\
\text { aphA3) }\end{array}$ & Commichau et al., 2008 \\
\hline GP804 & $\operatorname{trpC} 2$ gudB1 amyE::(gltA-lacZ aphA3) & Commichau et al., 2008 \\
\hline GP807 & $\operatorname{trpC} 2 \Delta g l t A B:: t e t$ & LFH-PCR $\rightarrow 168$ \\
\hline GP808 & $\operatorname{trpC} 2$ amyE::(gltA-lacZ aphA3) $\triangle$ gltAB::tet & pGP526 $\rightarrow$ GP807 \\
\hline GP809 & 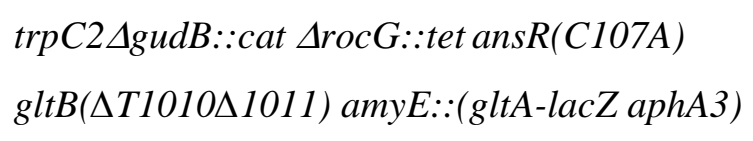 & LFH-PCR $\rightarrow$ GP717 \\
\hline GP810 & $\operatorname{trpC} \Delta \operatorname{\Delta rocG::tet}$ & LFH-PCR $\rightarrow 168$ \\
\hline GP811 & $\begin{array}{l}\operatorname{trp} C 2 \Delta g u d B:: c a t \text { rocG }:: \operatorname{Tn} 10 \text { spc } \\
\text { amyE::(gltA-lacZ aphA3) ansR::tet }\end{array}$ & $\begin{array}{l}\text { Flórez et al., subm.; } \\
\text { LFH } \rightarrow \text { GP28 }\end{array}$ \\
\hline GP812 & $\operatorname{trpC2}$ урьH::spec amyE::(gltA-lacZ cat) & $\begin{array}{l}\text { This work; } \\
\text { BD3349 } \rightarrow \text { GP669 }\end{array}$ \\
\hline
\end{tabular}




\begin{tabular}{|c|c|c|}
\hline Strain & Genotype $^{a}$ & Reference/Construction $^{\mathbf{b}}$ \\
\hline GP815 & $\begin{array}{l}\text { trpC2 amyE::(gltA-lacZ aphA3) lacR::Tn10 } \\
\text { spc gudB1 }\end{array}$ & $\begin{array}{l}\text { This work; } \\
\text { transposon mutagenesis } \\
\text { pIC333 } \rightarrow \text { GP804 }\end{array}$ \\
\hline GP816 & $\begin{array}{l}\operatorname{trpC} 2 \text { gudB1 gltC(C578T) amyE::(gltA-lacZ } \\
\text { aphA3) }\end{array}$ & $\begin{array}{l}\text { This work; } \\
\text { spontaneous mutation of } \\
\text { GP804 on C-Glc }\end{array}$ \\
\hline GP817 & $\begin{array}{l}\operatorname{trpC} 2 \text { gudB1 gltC(C578T) proJ::Tn10 spc } \\
\text { amyE::(gltA-lacZ aphA3) }\end{array}$ & $\begin{array}{l}\text { This work; } \\
\text { transposon mutagenesis } \\
\text { pIC333 } \rightarrow \text { GP816 }\end{array}$ \\
\hline GP819 & $\operatorname{trp} C 2$ gudB1 amyE::(gltA-lacZ cat) & $\begin{array}{l}\text { This work, } \\
\text { pGP919 } \rightarrow \text { GP804 }\end{array}$ \\
\hline GP1101 & $\operatorname{trpC} 2$ amyE::(gudB-lacZ cat) & $\begin{array}{l}\text { Gunka } \text { et al., subm.; } \\
\text { pGP651 } \rightarrow 168\end{array}$ \\
\hline GP1102 & $\operatorname{trp} C 2$ gudB1 amyE::(gudB-lacZ cat) & $\begin{array}{l}\text { Gunka et al., subm.; } \\
\text { pGP651 } \rightarrow \text { GP804 }\end{array}$ \\
\hline GP1103 & $\operatorname{trpC} 2$ rocG::Tn10 spc recA::erm cat & $\begin{array}{l}\text { Gunka et al., subm.; } \\
\text { IRN444 } \rightarrow \text { GP747 }\end{array}$ \\
\hline GP1104 & $\operatorname{trp} C 2$ rocG::Tn10 spc amyE::(gudB-lacZ cat) & $\begin{array}{l}\text { Gunka et al., subm.; } \\
\text { pGP651 } \rightarrow \text { GP747 }\end{array}$ \\
\hline GP1105 & $\begin{array}{l}\operatorname{trpC} 2 \text { rocG::Tn10 spc amyE::(gudB-lacZ cat) } \\
\text { gudB1 }\end{array}$ & $\begin{array}{l}\text { Gunka } \text { et al., subm.; } \\
\text { spontaneous mutation of } \\
\text { GP1104 on SP }\end{array}$ \\
\hline GP1106 & $\operatorname{trp} C 2 \triangle a d d A B:: s p c$ & $\begin{array}{l}\text { Gunka et al., subm.; } \\
\text { HVS666 } \rightarrow 168\end{array}$ \\
\hline GP1107 & $\begin{array}{l}\operatorname{trp} C 2 \triangle a d d A B:: \operatorname{spc} \operatorname{roc} G:: c a t \text { amyE::(gltA- } \\
\text { lacZ aphA3) }\end{array}$ & $\begin{array}{l}\text { Gunka et al., subm.; } \\
\text { GP1106 } \rightarrow \text { GP754 }\end{array}$ \\
\hline GP1123 & $\operatorname{trp} C$ amyE::(alf-aphA3 lacZ cat) & $\begin{array}{l}\text { Gunka et al., subm.; } \\
\text { pGP655 } \rightarrow 168\end{array}$ \\
\hline GP1127 & $\operatorname{trpC2}$ amyE::(alf1-aphA3 lacZ cat) & $\begin{array}{l}\text { Gunka et al., subm.; } \\
\text { spontaneous mutation of } \\
\text { GP1123 on SP-Kan }\end{array}$ \\
\hline
\end{tabular}




\begin{tabular}{|c|c|c|}
\hline Strain & Genotype $^{a}$ & Reference/Construction $^{\mathbf{b}}$ \\
\hline GP1152 & trpC2 $\Delta a n s R:: t e t$ & $\begin{array}{l}\text { This work; } \\
\text { GP811 } \rightarrow 168\end{array}$ \\
\hline GP1153 & $\operatorname{trp} C 2 \triangle a n s A B:: \mathrm{erm}^{C}$ & $\begin{array}{l}\text { This work; } \\
\text { LFH } \rightarrow 168\end{array}$ \\
\hline GP1154 & $\begin{array}{l}\operatorname{trp} C 2 \Delta \text { gudB::cat rocG::Tn10 spc } \\
\text { amyE::(gltA-lacZ aphA3) ansR (C107A )gltB } \\
(\Delta T 1010 \Delta 1011) \Delta \text { ansAB:: erm }{ }^{C}\end{array}$ & $\begin{array}{l}\text { Flórez et al., subm.; } \\
\text { LFH } \rightarrow \text { GP717 }\end{array}$ \\
\hline GP1155 & $\operatorname{trp} C 2$ amyE::(ansA-lacZ cat) & $\begin{array}{l}\text { This work; } \\
\text { pGP872 } \rightarrow 168\end{array}$ \\
\hline GP1156 & $\operatorname{trpC}$ CansR::tet amyE:(ansA-lacZ cat) & $\begin{array}{l}\text { This work; } \\
\text { pGP872 } \rightarrow \text { GP1152 }\end{array}$ \\
\hline GP1157 & $\operatorname{trp} C 2 \operatorname{roc} G:: c a t$ & $\begin{array}{l}\text { This work; } \\
\text { GP754 } \rightarrow 168\end{array}$ \\
\hline GP1158 & $\operatorname{trpC} 2$ gltC::Tn10 spc rocG::cat & $\begin{array}{l}\text { This work; } \\
\text { GP738 } \rightarrow \text { GP1157 }\end{array}$ \\
\hline GP1159 & $\operatorname{trpC2} \Delta h t p G:: c a t$ & $\begin{array}{l}\text { This work; } \\
\text { SV01 } \rightarrow \text { GP168 }\end{array}$ \\
\hline GP1160 & $\operatorname{trpC} 2$ $\triangle g u d B:: a p h A 3$ & $\begin{array}{l}\text { Gunka et al., subm.; } \\
\text { LFH } \rightarrow 168\end{array}$ \\
\hline GP1161 & $\operatorname{trp} C 2 \Delta$ gudB::aphA3 rocG::Tn10 spc & $\begin{array}{l}\text { Gunka et al., subm.; } \\
\text { GP1160 } \rightarrow \text { GP747 }\end{array}$ \\
\hline GP1162 & $\operatorname{trp} C 2 \Delta$ gudB::cat rocG::Tn10 $\triangle \operatorname{rocF}:: a p h A 3$ & $\begin{array}{l}\text { This work; } \\
\text { GP655 } \rightarrow \text { GP1199 }\end{array}$ \\
\hline GP1163 & $\begin{array}{l}\operatorname{trp} C 2 \Delta g u d B:: a p h A 3 \text { rocG::Tn10 spc } \\
\text { amyE::(gudB lacZ cat) }\end{array}$ & $\begin{array}{l}\text { Gunka et al., subm.; } \\
\text { pGP900 } \rightarrow \text { GP1161 }\end{array}$ \\
\hline GP1164 & $\operatorname{trp} C 2 \Delta$ gudB::cat rocG::Tn10 $\Delta$ rocD::aphA3 & $\begin{array}{l}\text { This work; } \\
\text { GP656 } \rightarrow \text { GP1199 }\end{array}$ \\
\hline GP1165 & $\begin{array}{l}\operatorname{trp} C 2 \text { } \triangle \text { gudB::aphA3 rocG::Tn10 spc } \\
\text { amyE::(gudB1-lacZ cat) }\end{array}$ & $\begin{array}{l}\text { This work; } \\
\text { spontaneous mutation } \\
\text { of GP1163 on SP }\end{array}$ \\
\hline
\end{tabular}




\begin{tabular}{|c|c|c|}
\hline Strain & Genotype $^{\text {a }}$ & Reference/Construction $^{\mathbf{b}}$ \\
\hline GP166 & $\operatorname{trpC2}$ rocG::Tn10 spc $\Delta h t p G:: c a t$ & $\begin{array}{l}\text { This work; } \\
\text { GP747 } \rightarrow \text { GP1159 }\end{array}$ \\
\hline GP1167 & $\operatorname{trp} C 2 \Delta m f d:: e r m C$ & $\begin{array}{l}\text { Gunka et al., } 2010 \text { subm.; } \\
\text { LFH } \rightarrow 168\end{array}$ \\
\hline GP1168 & $\begin{array}{l}\operatorname{trp} C 2 \triangle m f d:: e r m C \text { amyE::(alf-aphA3 lacZ } \\
\text { cat) }\end{array}$ & $\begin{array}{l}\text { Gunka } \text { et al., } 2010 \text { subm.; } \\
\text { GP1167 } \rightarrow \text { GP1123 }\end{array}$ \\
\hline GP1169 & $\operatorname{trp} C 2 \operatorname{roc} G:: \operatorname{Tn} 10$ spc $\Delta m f d:: \operatorname{erm} C$ & $\begin{array}{l}\text { Gunka et al., } 2010 \text { subm.; } \\
\text { GP1167 } \rightarrow \text { GP747 }\end{array}$ \\
\hline GP1171 & $\operatorname{trp} C 2$ xkdE::(N-yfp ermC) & $\begin{array}{l}\text { This work; } \\
\text { pGP886 } \rightarrow 168\end{array}$ \\
\hline GP1172 & $\operatorname{trp} C 2$ xkdE::(N-yfp emrC) & $\begin{array}{l}\text { This work; } \\
\text { pGP887 } \rightarrow 168\end{array}$ \\
\hline GP1173 & $\operatorname{trpC} 2$ lacA::(C-yfp aphA3) & $\begin{array}{l}\text { This work; } \\
\text { pGP888 } \rightarrow 168\end{array}$ \\
\hline GP1174 & $\operatorname{trp} C 2$ lacA::(C-yfp aphA3) & $\begin{array}{l}\text { This work; } \\
\text { pGP889 } \rightarrow 168\end{array}$ \\
\hline GP1175 & $\operatorname{trp} C 2 \Delta u v r A B::$ erm $C$ & $\begin{array}{l}\text { This work; } \\
\text { LFH } \rightarrow 168\end{array}$ \\
\hline GP1176 & $\operatorname{trp} C 2 \operatorname{roc} G:: \operatorname{Tn} 10$ spc $\triangle u v r A B:: \operatorname{erm} C$ & $\begin{array}{l}\text { This work; } \\
\text { GP1175 } \rightarrow \text { GP747 }\end{array}$ \\
\hline GP1177 & $\begin{array}{l}\operatorname{trp} C 2 \Delta \text { gudB::aphA3 amyE::(gudB } B_{\text {mut } 1} \text { lacZ } \\
\text { cat })\end{array}$ & $\begin{array}{l}\text { Gunka et al., subm.; } \\
\text { pGP1714 } \rightarrow \text { GP1160 }\end{array}$ \\
\hline GP1178 & $\begin{array}{l}\operatorname{trp} C 2 \Delta \text { gudB::aphA3 amyE::(gudB } B_{\text {mut } 2} \text { lacZ } \\
\text { cat })\end{array}$ & $\begin{array}{l}\text { Gunka et al., subm.; } \\
\text { pGP1715 } \rightarrow \text { GP1160 }\end{array}$ \\
\hline GP1179 & $\begin{array}{l}\operatorname{trp} C 2 \Delta \text { gudB::aphA3 rocG::Tn10 spc amyE:: } \\
\left(\text { gudB }{ }_{\text {mut }} \text { lacZ } \text { cat }\right)\end{array}$ & $\begin{array}{l}\text { Gunka et al., subm.; } \\
\text { GP747 } \rightarrow \text { GP1177 }\end{array}$ \\
\hline GP1180 & $\begin{array}{l}\operatorname{trp} C 2 \Delta \text { gudB::aphA3 rocG::Tn10 spc amyE:: } \\
\left(\text { gudB }{ }_{\text {mut } 2} \text { lacZ } \text { cat }\right)\end{array}$ & $\begin{array}{l}\text { Gunka et al., subm.; } \\
\text { GP747 } \rightarrow \text { GP1178 }\end{array}$ \\
\hline GP1188 & 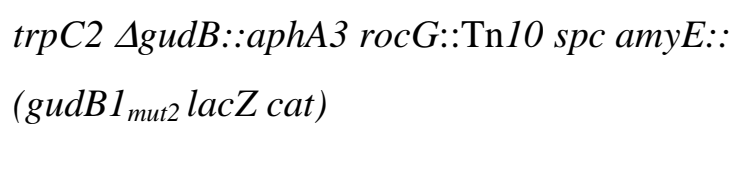 & $\begin{array}{l}\text { This work; } \\
\text { spontaneous mutation of } \\
\text { GP1180 on SP }\end{array}$ \\
\hline
\end{tabular}




\begin{tabular}{|c|c|c|}
\hline Strain & Genotype $^{\text {a }}$ & Reference/Construction \\
\hline GP1189 & $\operatorname{trp} C 2 \Delta u v r A B:: \operatorname{erm} C \operatorname{roc} G:: \operatorname{Tn} 10$ spc gudB1 & $\begin{array}{l}\text { This work; } \\
\text { spontaneous mutation of } \\
\text { GP1176 on SP }\end{array}$ \\
\hline GP1190 & $\operatorname{trpC} 2 \Delta m u t S L:: a p h A 3$ & This work; LFH $\rightarrow 168$ \\
\hline GP1191 & $\operatorname{trpC} 2 \operatorname{roc} G:: \operatorname{Tn} 10$ spc $\Delta m u t S L:: a p h A 3$ & $\begin{array}{l}\text { This work; } \\
\text { GP1191 } \rightarrow \text { GP747 }\end{array}$ \\
\hline GP1192 & $\begin{array}{l}\operatorname{trp} C 2 \operatorname{roc} G:: \operatorname{Tn} 10 s p c \\
\Delta m u t S L:: a p h A 3 \Delta u v r A B:: \text { ermC }\end{array}$ & $\begin{array}{l}\text { This work; } \\
\text { GP1176 } \rightarrow \text { GP1191 }\end{array}$ \\
\hline GP1193 & $\operatorname{trp} C 2 \operatorname{roc} G::$ cat gudB1 & $\begin{array}{l}\text { This work; } \\
\text { spontaneous mutation of } \\
\text { GP1 } 157 \text { on SP }\end{array}$ \\
\hline GP1194 & $\operatorname{trp} C 2 \operatorname{gudB}-3 x$ Flag $s p c$ & $\begin{array}{l}\text { This work; } \\
\text { pGP1720 } \rightarrow 168\end{array}$ \\
\hline GP1195 & $\operatorname{trpC} 2$ gudB1 -3xFlag spc & $\begin{array}{l}\text { This work; } \\
\text { pGP1720 } \rightarrow \text { GP804 }\end{array}$ \\
\hline GP1196 & $\operatorname{trp} C 2 \operatorname{roc} G:: T n 10$ spc $\triangle \operatorname{roc} F:: a p h A 3$ & $\begin{array}{l}\text { This work; } \\
\text { GP747 } \rightarrow \text { GP655 }\end{array}$ \\
\hline GP1197 & $\begin{array}{l}\operatorname{trp} C 2 \Delta \text { gudB::aphA3 rocG::Tn10 spc amyE:: } \\
\left(\text { gudB }{ }_{\text {mut } 3} \text { lacZ } \text { cat }\right)\end{array}$ & $\begin{array}{l}\text { Gunka } \text { et al., subm.; } \\
\text { pGP1721 } \rightarrow \text { GP1161 }\end{array}$ \\
\hline GP1198 & $\begin{array}{l}\operatorname{trpC} 2 \text { gudB::aphA3 rocG::Tn10 spc amyE:: } \\
\left(\text { gudB } 1_{\text {mut } 3} \text { lacZ } \text { cat }\right)\end{array}$ & $\begin{array}{l}\text { Gunka et al., subm.; } \\
\text { spontaneous mutation of } \\
\text { GP1197 on SP }\end{array}$ \\
\hline GP1199 & $\operatorname{trp} C 2 \Delta$ gudB::cat rocG:: $\operatorname{Tn} 10$ & $\begin{array}{l}\text { This work; } \\
\text { GP28 } \rightarrow 168\end{array}$ \\
\hline GP1200 & $\operatorname{trp} C 2 \operatorname{roc} G:: T n 10$ spc $\triangle$ rocD $:: a p h A 3$ & $\begin{array}{l}\text { This work; } \\
\text { GP747 } \rightarrow \text { GP656 }\end{array}$ \\
\hline HVS666 & $\operatorname{trp} C 2 \triangle a d d A B:: s p c$ & $\begin{array}{l}\text { Gunka } \text { et al., subm.; } \\
\text { Chédin } \text { et al., } 1998\end{array}$ \\
\hline IRN444 & $\operatorname{trpC} 2$ recA::ermC cat & Lemon et al., 2001 \\
\hline SV01 & leuA8 metB5 trpC2 hsrM1 $\Delta$ htpG::cat & Versteeg et al., 1999 \\
\hline
\end{tabular}




\begin{tabular}{|c|c|c|}
\hline Strain & Genotype $^{a}$ & Reference/Construction \\
\hline \multicolumn{3}{|c|}{ Escherichia coli } \\
\hline $\begin{array}{l}\text { BL21 } \\
\text { (DE3)/ } \\
\text { pLysS }\end{array}$ & $\begin{array}{l}\text { F- lon ompT rBmB hsdS gal (cIts857ind1 } \\
\text { Sam7 nin5 lacUV5- T7 gene1) }\end{array}$ & Sammbrock et al., 1989 \\
\hline BTH101 & $\begin{array}{l}\text { F- cya-99 araD139 galE15 galK16 rpsL1 } \\
\text { (StrR) hsdR2 mcrAl mcrB1 }\end{array}$ & Karimova et al., 2005 \\
\hline DH5 $\alpha$ & 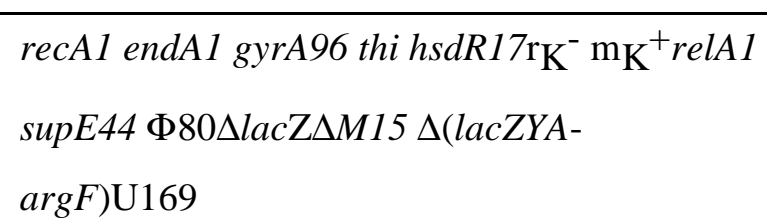 & Sammbrock et al., 1989 \\
\hline
\end{tabular}

XL1-Red endA1 gyrA96 thi- 1 hsdR17 supE44 relAl lac Greener \& Callahan, 1994 mutD5 mutS mutT Tn10 (tet)

\footnotetext{
a Resistance gene abbreviations as follows: aphA3, kanamycin; cat, chloramphenicol; erm $C$, erythromycin; $s p c$, spectinomycin; tet, tetracycline.

${ }^{b}$ Arrows indicate construction by transformation.
} 


\section{Curriculum vitae}

\section{Persönliche Angaben}

Name

Geburtsdatum, -ort

Staatsangehörigkeit

\section{Schulbildung}

$1988-1992$

$1992-1994$

$1994-2001$

\section{Studium}

04/2002 - 09/2002

$10 / 2002-01 / 2007$

02/2007 - 11/2007

\section{Promotion}

$01 / 2008-02 / 2011$

\author{
Katrin Gunka \\ 08.09.1981 in Cuxhaven \\ deutsch
}

Orientierungstufe Franzenburg

Abitur am Lichtenberg-Gymnasium Cuxhaven

Lehramtsstudium für die Sekundarstufe II,

Georg-August-Universität Göttingen

Diplomstudium Biologie,

Georg-August-Universität Göttingen

Anfertigung der Diplomarbeit

„Isolation von Mutationen, die die regulatorische

Wechselwirkung von RocG und GltC in Bacillus

subtilis beeinträchtigen“

Arbeitsgruppe von Prof. Dr. Jörg Stülke,

Institut für Mikrobiologie und Genetik, Georg-

August-Universität Göttingen

Promotionsthema: „Der Einfluss der Glutamatdehydrogenasen auf die Verknüpfung des

Kohlenstoff- und Stickstoffstoffwechsels in Bacillus subtilis“

Arbeitsgruppe von Prof. Dr. Jörg Stülke 